United States Department of Commerce
National Institute of Standards and Technology

NISTIR 3959

HEAT TRANSFER

IN A COMPACT HEAT EXCHANGER

CONTAINING RECTANGULAR CHANNELS

AND USING HELIUM GAS

Douglas A. Olson 


\section{NISTIR 3959}

\section{HEAT TRANSFER \\ IN A COMPACT HEAT EXCHANGER CONTAINING RECTANGULAR CHANNELS AND USING HELIUM GAS}

Douglas A. Olson

Chemical Engineering Science Division

Center for Chemical Engineering

National Measurement Laboratory

National Institute of Standards and Technology

Boulder, Colorado 80303-3328

January 1991

Sponsored by

National Aeronautics and Space Administration

Langley Research Center

Hampton, Virginia 23665

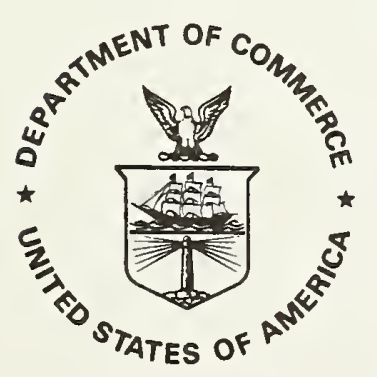

U.S. DEPARTMENT OF COMMERCE, Robert A. Mosbacher, Secretary NATIONAL INSTITUTE OF STANDARDS AND TECHNOLOGY, John W. Lyons, Director 

Page
Pag

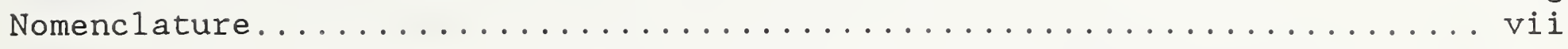

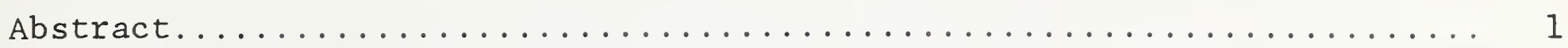

1. Introduction. .............................. 2

2. Description of experimental apparatus................... 2

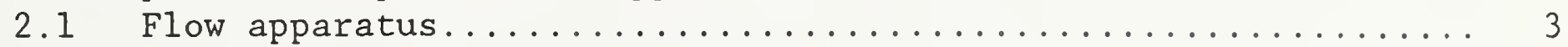

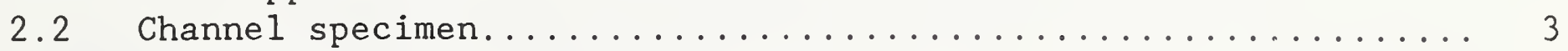

2.3 Instrumentation............................. 4

3. Description of experiments and analysis techniques............ 5

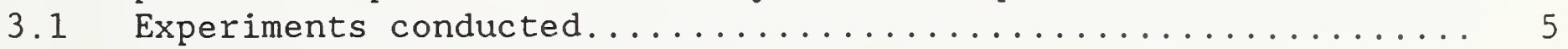

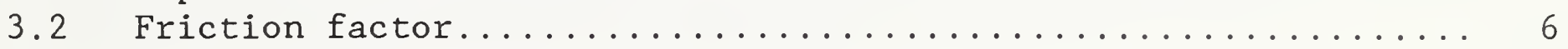

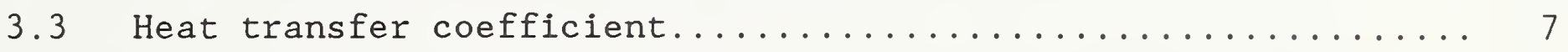

3.4 Uncertainty analysis........................ 10

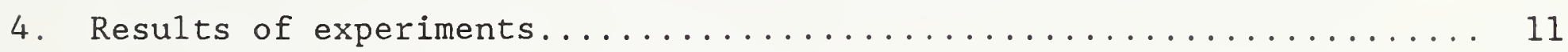

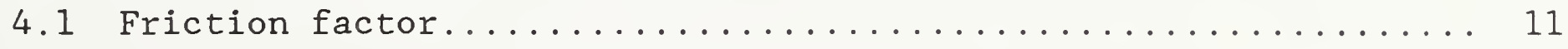

4.2 Temperature distributions and heat transfer............. 12

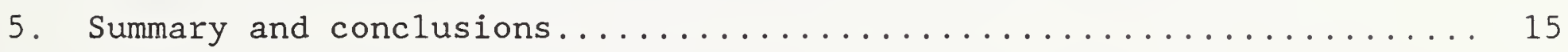

6. $\operatorname{References..\ldots \ldots \ldots \ldots \ldots \ldots \ldots \ldots \ldots \ldots \ldots \ldots \ldots \ldots \ldots \ldots .\ldots \ldots } 16$

\section{Appendices}

A. Heat flux distribution in reflective furnace.............. 17

B. Method of calculating flow distribution in specimen.......... 18 


\section{LIST OF TABLES}

$\underline{\text { Table }}$

1. Uncertainties in experimental measurements and gas properties at a

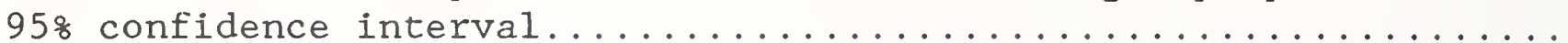

2. Summary of geometrical parameters and experimental conditions for

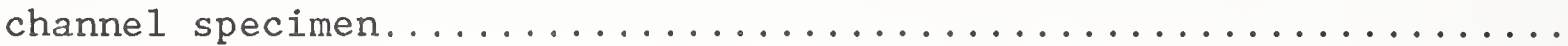

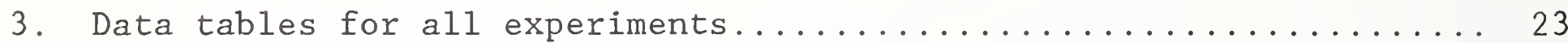

4. Uncertainties in data analysis parameters and calculated

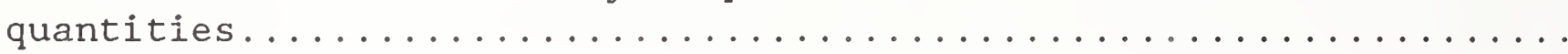

5. Predicted flow distribution in heat transfer experiments using method of Appendix B. 


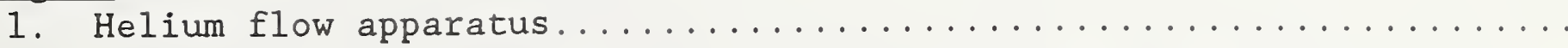

2. Specimen furnace, showing location of inlet gas temperature (A), upstream pressure (0), outlet gas temperature (B), and downstream

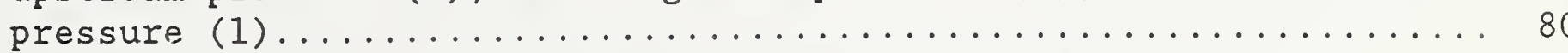

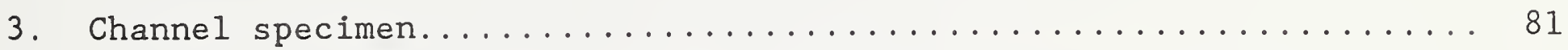

4. Top view of channel specimen showing locations of blockage in flow

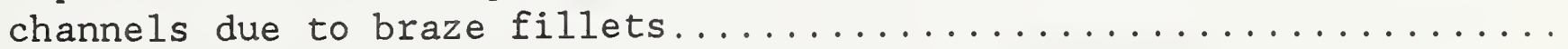

5. Friction factor ( $f$ ) as a function of Reynolds number (Re) for experiments 1 and 2 , no heating, compared to tube specimen

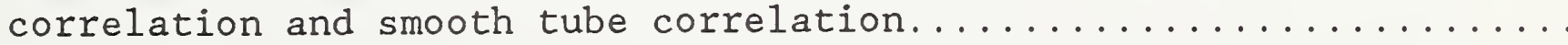

6. Percent difference between predicted and measured pressure drop $\left(P_{0}-P_{1}\right)$ as a function of helium flow rate $(\dot{m})$ for heat transfer

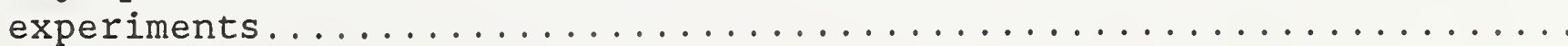

7. Wall $\left(\mathrm{T}_{\mathrm{w}}\right)$ and gas $\left(\mathrm{T}_{\hat{1}}\right)$ temperatures as a function of $\mathrm{x} / \mathrm{L}$; experiment $8,13.8 \mathrm{~kg} / \mathrm{h}$ helium flow, and $\mathrm{y} / \mathrm{W}=-0.04 \ldots \ldots \ldots \ldots$

8. Wall temperature $\left(T_{w}\right)$ as a function of $y / W$ at several $\mathrm{x} / \mathrm{L}$ locations;

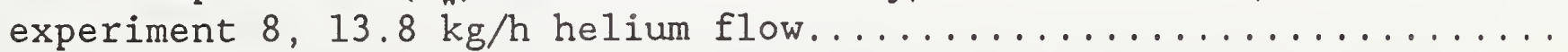

9. Wall-to-gas temperature difference $\left(T_{w}-T_{f}\right)$ and heat transfer coefficient (h) as a function of $\mathrm{x} / \mathrm{L}$; experiment $8,13.8 \mathrm{~kg} / \mathrm{h}$ helium

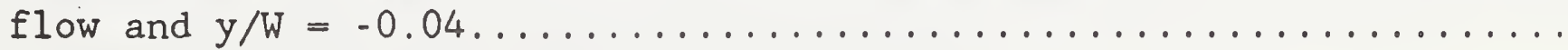

10. Reynolds number ( $\mathrm{Re}$ ), Nusselt number ( $\mathrm{Nu}$ ), and modified Nusselt number $\left(\mathrm{Nu}_{\mathrm{m}}\right)$ as a function of $\mathrm{x} / \mathrm{L}$; experiment $8,13.8 \mathrm{~kg} / \mathrm{h}$ helium

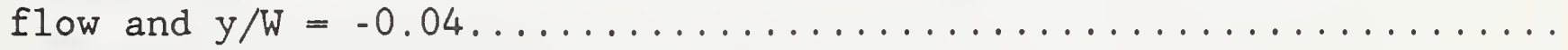

11. Modified Nusselt number $\left(\mathrm{Nu}_{\mathrm{m}}\right)$ as a function of Reynolds number (Re); all heated experiments with $0.2<\mathrm{x} / \mathrm{L}<0.8$ and $\mathrm{y} / \mathrm{W}=-0.04 \ldots \ldots \ldots$

A.1. Normalized meter heat flow as a function of $\mathrm{x} / \mathrm{L}$ for $\mathrm{y} / \mathrm{w}=0.12$, 368 voltage, reflective furnace calibration................ 90

B.1. Ratio of predicted channel flow to average channel flow, as a function of $\mathrm{y} / \mathrm{W}$ at several total flow rates for experiment $8 \ldots \ldots .$. 

$\mathrm{a}=$ coefficient of $\mathrm{Re}$ in $\mathrm{Nu}$ vs $\mathrm{Re}$ and $\mathrm{Pr}$ correlation

$A=$ inlet manifold location

$A_{c}=$ channel normal area $=h_{c} W_{c}$

$A_{f}=$ flow normal area $=n_{c} W_{c}$

$A_{n}=$ specimen normal area $=L \cdot W$

$A_{w}=$ wetted wall area (total wall area exposed to fluid) $=2 n\left(w_{c}+h_{c}\right) L$

$\mathrm{b}=$ coefficient of $\mathrm{Pr}$ in $\mathrm{Nu}$ vs $\mathrm{Re}$ and $\mathrm{Pr}$ correlation

$B=$ outlet manifold location

$c$ = leading coefficient in $\mathrm{Nu}$ vs $\mathrm{Re}$ and $\mathrm{Pr}$ correlation

$c_{p}=$ specific heat at constant pressure

$D_{h}=$ specimen hydraulic diameter $=2 \mathrm{w}_{\mathrm{c}} \mathrm{h}_{\mathrm{c}} /\left(\mathrm{w}_{\mathrm{c}}+\mathrm{h}_{\mathrm{c}}\right)$

$\mathrm{f}$ = friction factor

$\mathrm{f}_{\mathrm{q}}=$ heat flux distribution function

$\mathrm{G}=$ mass flow rate per unit flow normal area in channel $=\dot{\mathrm{m}}_{\mathrm{c}} / \mathrm{A}_{\mathrm{c}}=\rho \mathrm{V}$

$h=$ heat transfer coefficient

$\mathrm{h}=$ enthalpy

$h_{c}=$ height of channel

$\mathrm{k}=$ thermal conductivity

$\mathrm{L}=$ heated length of specimen

$\dot{\mathrm{m}}=$ mass flow rate

$\dot{\mathrm{m}}_{\mathrm{c}}=$ mass flow rate per channel

$\mathrm{n}=$ number of channels

$\mathrm{Nu}=$ Nusselt number $=\mathrm{h} \cdot \mathrm{D}_{\mathrm{h}} / \mathrm{k}$

$N u_{m}=$ modified Nusselt number $=\mathrm{Nu} \cdot\left(\mathrm{T}_{\mathrm{w}} / \mathrm{T}_{\mathrm{f}}\right)^{0.55}$

$P=$ pressure

$\operatorname{Pr}=$ Prandtl number $=\mu \cdot \mathrm{c}_{\mathrm{p}} / \mathrm{k}$

$\mathrm{q}_{\mathrm{n}}=$ local normal heat flux

$\mathrm{Q}_{\mathrm{px}}=$ fraction of total heat flow on specimen added up to position $\mathrm{x}$

= integration of furnace calibration function $\mathrm{f}_{\mathrm{q}}$, 0 to $\mathrm{x}$

$\mathrm{Q}_{\mathrm{T}}=$ total heat transfer to specimen

$\mathrm{q}_{\mathrm{w}}=$ local heat flux (heat flow per unit area) into the cooling fluid based on total wetted-wall area of the specimen

$r=$ recovery factor $=\mathrm{Pr}^{1 / 3}$ for turbulent flow

$\operatorname{Re}=$ Reynolds number $=\rho \mathrm{VD}_{\mathrm{h}} / \mu$

$\mathrm{T}=$ temperature

$\mathrm{T}_{\mathrm{aw}}=$ cooling fluid adiabatic wall temperature

$\mathrm{T}_{\mathrm{f}}=$ local bulk fluid temperature

$\mathrm{T}_{\mathrm{W}}=$ specimen wall temperature

$\mathrm{V}=$ velocity

$\mathrm{V}_{\mathrm{f}}=$ heater voltage

$\mathrm{W}=$ width of specimen

$\mathrm{w}_{\mathrm{c}}=$ width of channel

$W_{f}=$ uncertainty in friction factor

$\mathrm{W}_{\mathrm{h}}=$ uncertainty in heat transfer coefficient

$W_{n u}=$ uncertainty in Nusselt number

$W_{\text {qt }}=$ uncertainty in total heat transfer

$\mathrm{W}_{r e}=$ uncertainty in Reynolds number

$W_{t f}=$ uncertainty in fluid temperature

$W_{t w}=$ uncertainty in wall temperature 


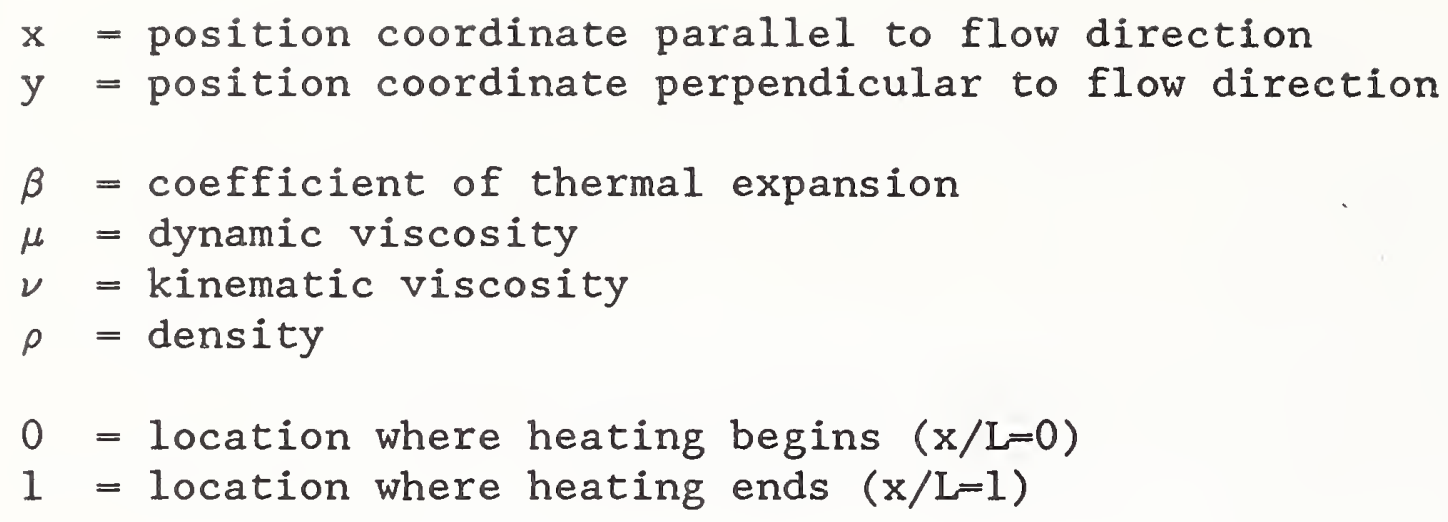




\title{
Heat Transfer in a Compact Heat Exchanger Containing Rectangular Channels And Using Helium Gas
}

\author{
Douglas A. Olson \\ Chemical Engineering Science Division \\ National Institute of Standards and Technology \\ Boulder, CO 80303-3328
}

\begin{abstract}
We have constructed a compact heat exchanger consisting of 12 parallel, rectangular channels in a flat piece of commercially pure nickel. This channel specimen was radiatively heated on the top side at heat fluxes of up to $77 \mathrm{~W} / \mathrm{cm}^{2}$, insulated on the back side, and cooled with helium gas flowing in the channels at 3.5 to $7.0 \mathrm{MPa}$ and Reynolds numbers of 1400 to 28000 . The measured friction factor was lower than that of the accepted correlation for fully developed turbulent flow, although our uncertainty was high due to uncertainty in the channel height and a high ratio of dynamic pressure to pressure drop. The measured Nusselt number, when modified to account for differences in fluid properties between the wall and the cooling fluid, agreed with past correlations for fully developed turbulent flow in channels. Flow nonuniformity from channel-to-channel was as high as $12 \%$ above and $19 \%$ below the mean flow.

Key words: apparatus; compact heat exchanger; convection heat transfer; friction factor; high temperature; National Aerospace Plane; radiative furnace; rectangular channel; turbulent flow; variable property effects.
\end{abstract}

This work was supported by NASA Langley Research Center under contract L7400C. 


\section{Introduction}

Development of a National Aerospace Plane (NASP), which will fly at hypersonic speeds, requires novel cooling techniques to manage the anticipated high heat fluxes on various components (Shore, 1986). The problem that motivates this work is cooling of the engine struts. Due to aerodynamic heating associated with the combustion of the hydrogen fuel, along with thermal radiation from the fuel combustion, the engine struts are expected to receive a normal heating load in excess of $2000 \mathrm{~W} / \mathrm{cm}^{2}$ (Scotti et al., 1988). NASA plans to cool the struts by attaching a cooling jacket heat exchanger to the surface facing the high heat flux. Hydrogen gas will flow through the cooling jacket and absorb the heat before entering the engine. The anticipated conditions are that the hydrogen gas will enter the heat exchangers at $56 \mathrm{~K}$ and $6.9 \mathrm{MPa}$ (1000 psi), and exit at $890 \mathrm{~K}$ and $4.8 \mathrm{MPa}$ (700 psi). The heat exchangers are expected to be thin ( $6 \mathrm{~mm}$ or less) perpendicular to the flow direction to add minimal weight and thickness to the struts. Small flow passages will also produce high rates of convective heat transfer, which will reduce the exchanger temperatures. Reynolds numbers are expected to be in the range 10000 to 30 000, with the variation due to the flow rate and the specific design of the flow passage.

In order to test heat exchangers developed by NASA, we constructed an apparatus which can provide helium gas flow and a well-characterized heat flux to a heat exchanger specimen (01son, 1989). This apparatus was first used to test a "tube specimen", which consisted of 20 nickel tubes, $1 \mathrm{~mm}$ ID, 1ying in parallel on a nickel base plate (Olson and Glover, 1990). For turbulent flow in the tube specimen and normal heat flux of up to $54 \mathrm{~W} / \mathrm{cm}^{2}$, the Nusselt numbers we measured were in good agreement with the Nusselt numbers for flow in a single tube with well-characterized boundary conditions. In this work we present the experimental results of a second possible heat exchanger configuration for the NASP cooling jacket. We also describe a modification to the flow apparatus, namely a new furnace, which reduced the experimental time constant. The specimen tested here has 12 parallel rectangular channels through which the helium flows. A rectangular channel has been proposed as a possible configuration for the NASP cooling jacket (Scotti, et al., 1988); the flow passage geometry of our specimen was chosen for ease of fabrication and instrumentation rather than for optimal thermal performance. The length and width of the specimen, flow manifold connections, and instrumentation were identical to those of the specimens to be constructed by NASA.

\section{Description of experimental apparatus}

The description which follows is based on Olson (1989) and 0lson and Glover (1990). The apparatus was designed to test a subset of the conditions required for the NASP application. Those conditions are (1) a heating rate of 0 to $80 \mathrm{~W} / \mathrm{cm}^{2}$; (2) an inlet temperature of $300 \mathrm{~K}$; (3) a cooling-gas pressure of up to $6.9 \mathrm{MPa}$ at the inlet; and (4) an outlet temperature of $810 \mathrm{~K}$ or less. We chose helium as the coolant gas because of the similarities in specific heat, thermal conductivity, and dynamic viscosity to the corresponding properties of hydrogen. In addition, helium does not have the explosive hazard of hydrogen. Because of the property similarities, the Reynolds 
number, Prandt1 number, and temperature rise from specimen inlet to outlet can be matched between helium and hydrogen.

\subsection{Flow apparatus}

The helium flow apparatus is shown in figure 1, with the details of the specimen furnace section in figure 2. Helium gas at $17 \mathrm{MPa}$ (2500 psi) or less was supplied from a tube trailer outside the laboratory. The tube trailer contained $1100 \mathrm{~m}^{3}$ of gas (STP). With valves 1 and 2 open, gas flowed from the trailer, through the inlet piping, and was filtered before entering the domeloaded pressure regulator (valve 3 ). The regulator set the flow pressure downstream of the regulator to the value of an external control pressure, either $7 \mathrm{MPa}$ or $3.5 \mathrm{MPa}$ (500 psi) for these experiments.

Within the furnace (fig. 2), the gas flowed into an inlet distribution manifold which directed it to the heat exchanger specimen. A similar distribution manifold collected the gas exiting the specimen and directed it to the outlet tubing. Gas pressure was measured at the pressure taps as shown at location 0 (start of heated zone) and at location 1 (end of heated zone). The specimen was located in the target area of the furnace $(7.8 \mathrm{~cm}$ wide by $15.2 \mathrm{~cm}$ long), which delivered radiant heat to the specimen and raised the temperature of the helium as it flowed through the specimen.

The furnace consisted of a high-intensity infrared radiant heater, surrounded by highly reflective walls which reflected the heat from the heater to the specimen. The reflective walls were made of $6.4 \mathrm{~mm}$ thick aluminum plates, polished on the inner surface, with a water-cooled cooling jacket soldered to the outside. This "reflective furnace" replaced the furnace of refractory walls which had been used for tests on the tube specimen (Olson and Glover, 1990). The heater contained six high-temperature infrared lamps mounted in an aluminum housing. A phase-angle power controller which used 480 VAC, single phase, and $75 \mathrm{~A}$ at maximum voltage powered the heater.

Downstream of the furnace section, the hot gas flowed through a cooling coil immersed in a water bath. The rate of gas flow was manually adjusted at the bath outlet by valve 4, which also dropped the gas pressure to atmospheric pressure. Beyond the valve, we measured helium flow rate with a heated-tube thermal mass flow meter. After exiting the flow meter the gas was vented outside the laboratory.

\subsection{Channel pecimen}

The channel specimen is shown in figure 3. It consisted of 12 parallel flow channels milled in a lower plate of commercially pure nickel (UNS 02200), with a cover plate of the same nickel brazed to it. The gas was directed into the channels by the inlet manifold, flowed down the channels, and was collected in the outlet manifold. Heating was from the top in figure 3 . The channel width and height were $3.18 \mathrm{~mm}$ and $0.56 \mathrm{~mm}$, respectively. The ridge between channels was $3.18 \mathrm{~mm}$ wide. The lower plate was $3.12 \mathrm{~mm}$ thick and the cover plate was $1.93 \mathrm{~mm}$ thick, for a total thickness of $5.05 \mathrm{~mm}$. The specimen was $7.86 \mathrm{~cm}$ wide and $19.1 \mathrm{~cm}$ long. The pressure taps were tubes, $1.5 \mathrm{~mm}$ OD and $1.0 \mathrm{~mm}$ ID, brazed into holes penetrating one of the channels. 
The cover and base plate were cut to size and the channels milled at NIST. The cover plate was brazed to the base plate at NASA Langley Research Center in a vacuum oven using a braze alloy foil of $50 \%$ gold, $25 \%$ palladium, and $25 \%$ nickel (AMS-4784, $1394 \mathrm{~K}$ liquidus). Prior to brazing, the inner facing surface of both plates was lapped to a flatness of $\pm 0.01 \mathrm{~mm}$. Figure 4 shows a sketch of an x-ray of the specimen after brazing. The figure shows that large braze fillets formed in 4 of the 12 channels, which partially occluded the flow passages.

The assembled specimen was brazed to slots in the inlet and outlet manifolds using a braze alloy of $82 \%$ gold and $18 \%$ nickel (AMS-4787, $1223 \mathrm{~K}$ liquidus). The pressure tap tubes were brazed to the specimen during the same braze cycle. We pressurized the manifold and specimen to $10.3 \mathrm{MPa}$ (1500 psi) prior to installing the instrumentation, and there were no leaks. We painted the top side of the specimen (the cover plate side) a flat black over the 15.2 $\mathrm{cm}$ length, to establish a uniform and highly absorptive surface over the heated area. The paint was rated to $1000^{\mathrm{K}}\left(1350^{\circ} \mathrm{F}\right)$.

\subsection{Instrumentation}

We measured the temperature of the gas in the inlet and outlet manifolds, gas pressure in the specimen, specimen temperatures, and the aforementioned gas flow rate. The measurement technique and uncertainties, along with the gas property uncertainties, are summarized in table 1.

We determined the distribution of heat flux on the specimen by calibrating the furnace prior to inserting the specimen. The heat flux distribution was defined as the local, normal (perpendicular) heat flux as a function of position over the furnace target. The heat flux was constant in the direction perpendicular to flow $(y)$, and varied by no more than $\pm 7 \%$ in the direction parallel to flow ( $x$ ) except within $6 \%$ of the end walls. Details of the furnace calibration are found in appendix $A$.

The gas inlet and outlet temperatures were measured with platinum resistance thermometers (PRTs), $4.8 \mathrm{~mm}$ diameter, inserted in the gas manifolds at locations $A$ and $B$ of figure 2. We measured the gas pressure at location 0 in the specimen with a variable-reluctance pressure transducer which had an output of $8.6 \mathrm{MPa}$ full scale. Difference in pressure between locations 0 and 1 in the specimen was measured with a differential pressure transducer, also a variable-reluctance type with an output of $140 \mathrm{kPa}$ (20 psi) full scale.

We measured specimen temperatures with thermocouples made from type-N wire, with a wire diameter of $0.25 \mathrm{~mm}$. We spot-welded 25 thermocouples to the side opposite the radiant heat flux (insulated-side). The heated-side temperature was measured at 7 locations with type-N thermocouples mounted as shown in figure 3. Two holes, $0.33 \mathrm{~mm}$ diameter, were drilled $1.27 \mathrm{~mm}$ on-center through the ridge between the channels. The holes were back-drilled to within $0.20 \mathrm{~mm}$ of the surface with a $0.57 \mathrm{~mm}$ diameter drill. We spot-welded each wire of the pair to the heated surface, with the lead extending out the hole on the insulated side. The thermocouple circuit was completed by the specimen material between the two wires. A quartz sleeve, $0.48 \mathrm{~mm}$ outer diameter, was 
inserted over the wire into the hole to electrically insulate the wire from the wall of the hole. Because a portion of the specimen was removed and replaced by wire plus quartz, each of which had a thermal conductivity lower than that of the specimen, mounting the thermocouple locally increased the specimen temperature. We estimated the magnitude of this temperature rise from a finite-element analysis as $2-5 \mathrm{~K}$ at a radiant heat flux of $50 \mathrm{~W} / \mathrm{cm}^{2}$. Temperatures measured with the insulated-side thermocouples were used to determine the heat transfer coefficient, as the installation technique did not disturb the specimen temperatures and conduction errors were insignificant.

All thermocouples were connected to an isothermal reference box. We measured the temperature of the reference box with a platinum resistance thermometer. Copper conductor wire connected the reference box to the data scanner. The connector box introduced negligible error in the temperature measurement (Olson, 1989).

All instrument signals were multiplexed through an automated scanner and measured with a digital voltmeter. The scanner and voltmeter were controlled with a personal computer through an IEEE-488 bus. Raw signals were stored on a hard disk and copied to floppy disk for backup. Signals were converted to SI units and the data analyzed at the completion of an experimental run. Some signal readings were converted immediately to SI units and displayed on the video terminal to assist in monitoring and operating the experiment. We have included the measurement uncertainties introduced by the data acquisition system in the stated uncertainties of each sensor.

\section{Description of experiments and analysis techniques}

\subsection{Experiments conducted}

A conditions for the nine experiments conducted with the channel specimen in the helium flow apparatus are summarized in table 2. Also listed are the values for the geometrical parameters required for the data analysis. Table 3 lists values for all the measured and calculated parameters at each data point for each experiment. Tests were conducted at system pressures of either 3.5 $\mathrm{MPa}$ (500 psi) or $7.0 \mathrm{MPa}(1000 \mathrm{psi})$. In experiments 1 and 2 , we tested a range of helium flow rates, without heating the specimen, to determine the friction factor. In experiments 3 to 9 , we varied the heater lamp voltage to vary the rate of specimen heating; at each heating rate a range of helium flow rates was tested up to $41 \mathrm{~kg} / \mathrm{h}$. The range in Reynolds number was 1400 to 28 000, while the range in normal heat flux was 0 to $77 \mathrm{~W} / \mathrm{cm}^{2}\left(68 \mathrm{Btu} /\left(\mathrm{s} \cdot \mathrm{ft}^{2}\right)\right)$. The minimum inlet gas temperature was $291 \mathrm{~K}\left(64^{\circ} \mathrm{F}\right)$, while the maximum gas outlet temperature was $710 \mathrm{~K}\left(818^{\circ} \mathrm{F}\right)$. The maximum specimen temperature we measured was $784 \mathrm{~K}\left(951^{\circ} \mathrm{F}\right)$.

Because of the high heat fluxes generated by the furnace, we carefully followed a procedure to prevent overheating the specimen during experimental set-up, run, and shut-down. With inadequate helium flow to cool the specimen, the furnace is capable of heating the specimen beyond the melting point of the brazing alloy and the nickel; with an internal pressure of $3.5 \mathrm{MPa}$ or greater this could easily rupture the specimen. We always started helium flow before 
turning on the furnace, and we maintained helium flow after the furnace was turned off. To set an experimental point, we closed valves 1 and 2 , set the control pressure on valve 3 , and cracked open valve 4 (see fig. 2). We opened valve 1 and verified that the tube trailer pressure was at least $25 \%$ above the desired system pressure. Then, we slowly opened valve 2 to full open to establish the helium flow. Valve 4 was adjusted to set a flow rate of at least $5 \mathrm{~kg} / \mathrm{h}$. Next, we turned on the furnace heater lamp to a low voltage (108) while monitoring temperatures. The lamp voltage was then turned up to the desired setting, and the helium flow was increased if necessary to provide sufficient cooling.

Before taking the first data point, we waited at least 15 minutes with the heater lamp at steady power to allow the specimen and manifolds to reach thermal steady-state. The reflective furnace reached thermal steady-state in about 5 min, but the outlet manifold had a longer time constant due to its large thermal capacitance. We scanned the sensors at least twice at each setting. After sampling all the sensors, we changed the helium flow rate by adjusting valve 4. At each new flow rate, we waited a minimum of 5 min to establish thermal steady-state before taking data, because a change of flow rate also affected gas, specimen, and manifold temperatures. After we finished taking data at one heater setting, we turned off the heater and reduced the helium flow to $5 \mathrm{~kg} / \mathrm{h}$ or less. We turned off the helium flow when the furnace had cooled sufficiently, usually after about $10 \mathrm{~min}$.

An unsteady experimental setting could translate into errors in the calculated performance parameters. In the data analysis to follow, we have assumed the settings were sufficiently steady to ignore thermal transients. steady setting was established by maintaining constant helium flow, gas pressure, furnace heating, and gas inlet temperature. All were held steady to within the uncertainty in the calibrations of the measurements. In the tube specimen tests (Olson and Glover, 1990), we observed the inlet gas temperature to decrease due to gas expansion. Because the present experiments were conducted in summer, the tubing between the helium supply trailer and the laboratory provided sufficient heat transfer to maintain a constant gas inlet temperature.

For the experiments conducted, we analyzed the data to determine the heat transfer coefficient, $h$, and the friction factor, $f$. The heat transfer coefficient was expressed as a dimensionless number, the Nusselt number, $\mathrm{Nu}$. A modified Nusselt number, $\mathrm{Nu}_{\mathrm{m}}$, was calculated to include the effects of variations in thermophysical properties, which we found to be significant in the tube specimen experiments. $\mathrm{Nu}$ and $\mathrm{Nu}_{\mathrm{m}}$ were correlated with the Reynolds number, $\mathrm{Re}$. The parameters $\mathrm{h}, \mathrm{Re}$, and $\mathrm{Nu}$ were calculated at each location of an insulated-side thermocouple.

\subsection{Friction factor}

The friction factor results from an integration of the one-dimensional momentum equation in the flow direction:

$$
P_{0}-P_{1}=G^{2}\left(1 / \rho_{1}-1 / \rho_{0}\right)+\left(2 G^{2} / D_{h}\right) \int_{0}^{1}(f / \rho) d x
$$




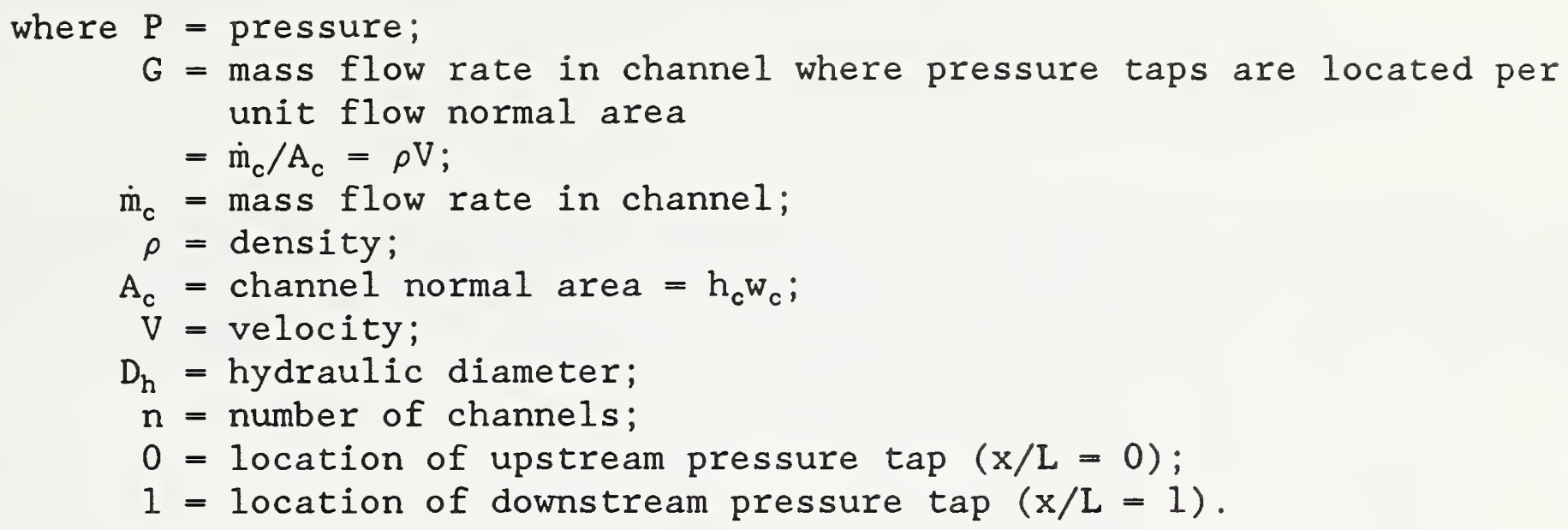

The first term on the right hand side of the equation is the pressure change due to flow acceleration, and the second term is the pressure drop due to frictional effects. Temperatures measured perpendicular to the flow direction for experiments with heating indicated that the flow was not evenly distributed in the 12 channels. The flow in the channel with the pressure taps was determined by the method described in appendix $B$.

If the change in density is small compared to the absolute density, and the pressure drop through the specimen is linear, then the integral can be approximated as a constant and the resulting equation for $f$ is

$$
f=\frac{P_{0}-P_{1}-G^{2}\left(1 / \rho_{1}-1 / \rho_{0}\right)}{2\left(G^{2} / \rho\right) \cdot\left(L / D_{h}\right)},
$$

with $\rho=\left(\rho_{0}+\rho_{1}\right) / 2$.

The density-change criterion was met when there was no heating, but when the specimen was heated the exit density was as small as half the entrance density, and eq (3) was not valid. Hence, the friction factor was determined for the tests with no heating as a function of Reynolds number, where

$$
\operatorname{Re}=\rho \mathrm{VD}_{\mathrm{h}} / \mu
$$

To determine whether specimen heating had an effect on the friction factor, eq (1) was integrated in a summation form from the inlet to outlet to predict the pressure drop $P_{0}-P_{1}$, for the experiments with specimen heating. The integral was evaluated at each location where wall temperature was measured, with $f$ found from the f-vs-Re correlation of experiments 1 and 2 and the local density found from the gas temperature and pressure. This predicted pressure drop was compared with the measured pressure drop.

\subsection{Heat transfer coefficient}

The heat transfer coefficient, $h$, is defined through the equation

$$
\mathrm{q}_{\mathrm{w}}=\mathrm{h} \cdot\left(\mathrm{T}_{\mathrm{w}}-\mathrm{T}_{\mathrm{aw}}\right)
$$

where $\mathrm{q}_{\mathrm{w}}=$ local heat flux (heat flow per unit area) into the cooling 
fluid based on total wetted-wall area of the specimen;

$\mathrm{h}=$ heat transfer coefficient;

$\mathrm{T}_{\mathrm{w}}=$ specimen wall temperature;

$\mathrm{T}_{\mathrm{aw}}=$ adiabatic wall temperature of the cooling fluid.

The adiabatic wall temperature is used in gas flows whenever the kinetic energy is significant compared to enthalpy changes (Rohsenow and Choi, 1961). Friction can cause the local wall temperature to exceed the bulk fluid temperature for an adiabatic specimen, and the adiabatic wall temperature approximates this effect. It is defined as

$$
\mathrm{T}_{\mathrm{aw}}=\mathrm{T}_{\mathrm{f}}+r \mathrm{~V}^{2} /\left(2 \mathrm{c}_{\mathrm{p}}\right)
$$

where $\mathrm{T}_{\mathrm{f}}=$ local bulk fluid temperature;

$r=$ recovery factor $=\operatorname{Pr}^{1 / 3}$ for turbulent flow.

Adiabatic heating was as much as $2 \mathrm{~K}$, and was greatest for the low pressure experiments at high flow rate and high heating rate. The local heat flux in eq (5) is expressed in terms of the total heat transfer to the specimen, $Q_{T}$, the total wetted wall area, and the furnace calibration function, $f_{q}$ (0lson, 1989), which is a dimensionless expression of the local normal heat flux:

$$
\begin{aligned}
\mathrm{q}_{\mathrm{w}} & =\left(\mathrm{Q}_{\mathrm{T}} / \mathrm{A}_{\mathrm{n}}\right) \cdot \mathrm{f}_{\mathrm{q}} \cdot\left(\mathrm{A}_{\mathrm{n}} / \mathrm{A}_{\mathrm{w}}\right), \\
\text { with } \mathrm{A}_{\mathrm{w}} & =\text { wetted wall area }=2 \mathrm{n}\left(\mathrm{w}_{\mathrm{c}}+\mathrm{h}_{\mathrm{c}}\right) \mathrm{L} ; \\
\mathrm{A}_{\mathrm{n}} & =\text { specimen heated normal area }=\mathrm{L} \cdot \mathrm{W} .
\end{aligned}
$$

The function $f_{q}$ is on the order of 1 , and if the heat flux were constant then $f_{q}$ would be 1 everywhere. The wall temperature used in eq (5) was measured with the thermocouples on the insulated side of the specimen. We have assumed that wall conduction was negligible both in the flow direction ( $x$ ) and perpendicular to the flow direction (y), and thus that at each position the heat incident on the specimen is all convected into the fluid.

The heat transfer coefficient, $h$, is defined in terms of the temperature of the insulated wall, because that temperature was measured with the least uncertainty. We desire to compare our results to the literature where $\mathrm{h}$ is defined in terms of a solid-fluid interface temperature. Because the specimen was heated from one side only and the solid thermal conductivity was finite, specimen temperatures will vary between the heated side, the insulated side, and the solid-fluid interface. However, because the Biot number (ratio of wall conduction resistance to fluid convection resistance) was less than 1 , temperature variations in the specimen should be much less than the temperature difference between the wall and the fluid. A finite-element conduction analysis using anticipated values of the heat transfer coefficient indicated that the wall temperature (at the solid-fluid interface) varied from $6.5 \mathrm{~K}$ greater than to $9.5 \mathrm{~K}$ less than the insulated side temperature (for 50 $\mathrm{W} / \mathrm{cm}^{2}$ hot side heat flux and $\left.\mathrm{h}=6400 \mathrm{~W} /\left(\mathrm{m}^{2} \cdot \mathrm{K}\right)\right)$. This compares with a temperature difference between the wall and bulk fluid of at least $67 \mathrm{~K}$ for the same conditions. No attempt was made to extrapolate the measured insulated-side temperature to a solid-fluid interface temperature. 
Combining eqs (5), (6) and (7) and rearranging, we get

$$
h=\frac{\left(Q_{T} / A_{w}\right) \cdot f_{q}}{\left\{T_{w}-\left[T_{f}+\left(r V^{2}\right) /\left(2 c_{p}\right)\right]\right\}} .
$$

The flow-direction energy equation was used to calculate $Q_{T}$ (to follow). Gas temperature $\mathrm{T}_{f}$ was calculated using the flow-direction energy equation along with the furnace calibration (also to follow).

The total heat absorbed by the specimen was calculated from the temperatures of the gas inlet and outlet, the helium flow rate, and the gas pressure drop. It was not necessary to adjust for a heat leak to or from the furnace, as the low temperatures of the reflective furnace made the term negligible. In our previous work with the tube specimen, the heat leak was 2 to 58 (Olson and Glover, 1990).

$$
\mathrm{Q}_{\mathrm{T}}=\dot{\mathrm{m}}\left(\mathrm{h}_{\mathrm{B}}-\mathrm{h}_{\mathrm{A}}\right) \text {, }
$$

where $\mathrm{h}=$ enthalpy;

$A=$ location in inlet manifold of PRT;

$B=$ location in outlet manifold of PRT.

We neglected kinetic energy changes from A to B because they were insignificant compared to the uncertainties of the temperature measurement. The change in enthalpy is given by

$$
h_{B}-h_{A}=c_{p} \cdot\left(T_{B}-T_{A}\right)+\int_{A}^{B}[(1-\beta T) / \rho] d P,
$$

where $\beta=$ coefficient of thermal expansion.

The specific heat is constant for helium for the range of conditions tested. The pressure term was included to account for the slight divergence from the ideal gas state for helium at these temperatures and pressures. The pressure at $A$ and $B$ is estimated by assuming a linear drop along the specimen and extrapolating the pressure from 0 and 1 . This assumption introduces less than 0.18 error in $\mathrm{Q}_{\mathrm{T}}$. The integral was evaluated using the virial equation of state for the gas (McCarty, 1973). Combining eqs (9) and (10) yields for $Q_{T}$ :

$$
Q_{T}=\dot{m} \cdot\left\{c_{p} \cdot\left(T_{B}-T_{A}\right)+\int_{A}^{B}[(1-\beta T) / \rho] d P\right\}
$$

The fluid temperature, $T_{f}$, was calculated by integrating the flow energy equation from the inlet manifold up to the location of interest (designated as $x)$, now including kinetic energy:

$$
\mathrm{T}_{\mathrm{fx}}=\mathrm{T}_{\mathrm{A}}+\frac{\mathrm{Q}_{\mathrm{T}} / \mathrm{n} \cdot \mathrm{Q}_{\mathrm{px}}}{\dot{\mathrm{m}}_{\mathrm{c}} \cdot \mathrm{c}_{\mathrm{p}}}+\frac{\int_{\mathrm{A}}^{\mathrm{x}}[(1-\beta \mathrm{T}) / \rho] \mathrm{dP}}{\mathrm{c}_{\mathrm{p}}}-\frac{\mathrm{V}_{\mathrm{x}}^{2}}{2 \mathrm{c}_{\mathrm{p}}}
$$




$$
\text { where } \begin{aligned}
\mathrm{Q}_{\mathrm{px}}= & \text { fraction of total heat flow on specimen added up to } \\
& \text { position } \mathrm{x} \text {; } \\
= & \text { integration of furnace calibration function } \mathrm{f}_{\mathrm{q}}, 0 \text { to } \mathrm{x} .
\end{aligned}
$$

Since the transverse temperature measurements for heated experiments indicate the flow has not split evenly into the 12 channels, we calculate a local channel flow rate according to appendix $B$. The fluid temperature requires the velocity at $x$, given by

$$
\mathrm{V}_{\mathrm{x}}=\dot{\mathrm{m}}_{\mathrm{c}} /\left(\mathrm{A}_{\mathrm{c}} \rho_{\mathrm{x}}\right)
$$

The density is given by the equation of state (McCarty, 1973) as

$$
\rho_{\mathrm{X}}=\rho_{\mathrm{X}}\left(\mathrm{T}_{\mathrm{fX}}, \mathrm{P}_{\mathrm{x}}\right)
$$

We assume the pressure varies linearly between 0 and 1:

$$
P_{x}=P_{0}-\left(P_{0}-P_{1}\right) \cdot x / L \text {. }
$$

The maximum error in $\mathrm{T}_{f}$ introduced by our assumption of a linear pressure variation is less than $0.02 \mathrm{~K}$.

With eq (15) substituted into eq (12) to evaluate the pressure term, eqs (12), (13), and (14) form a system of three equations in the unknowns of temperature, velocity, and density. They were solved through iteration.

With $\mathrm{T}_{f}$ and $\mathrm{V}$ determined at location $\mathrm{x}$, the heat transfer coefficient was calculated using eq (8). The Nusselt number, Prandtl number, and Reynolds number were then calculated, with the transport properties evaluated at the bulk fluid temperature, $\mathrm{T}_{\mathrm{f}}$ :

$$
\begin{aligned}
& \mathrm{Nu}=\mathrm{h} \cdot \mathrm{D}_{\mathrm{h}} / \mathrm{k}, \\
& \mathrm{Pr}=\mu \cdot \mathrm{c}_{\mathrm{p}} / \mathrm{k} .
\end{aligned}
$$

Transport properties were calculated from the functions given in McCarty (1972). The Nu, $\mathrm{Pr}$, and Re performance parameters assume constant fluid properties at the location $x$. Due to the large wall-to-fluid temperature difference, viscosity and thermal conductivity varied between the wall and the fluid (variation was as much as $26 \%$ for the lowest helium flow in experiment 8). We used the temperature ratio method of Rohsenow and Hartnett (1973) to correlate the data by calculating:

$$
N u_{m}=N u \cdot\left(T_{w} / T_{f}\right)^{0.55} \text {. }
$$

$\mathrm{Nu}_{\mathrm{m}}$ was then correlated with $\mathrm{Re}$.

3.4 Uncertainty analysis

Uncertainties for the calculated quantities were obtained by Taylor-series 
error propagation as described by ASME (1986). This technique generally produces the same level of confidence in a calculated result as the level of confidence in the measurements which contribute to the result (Kline and McClintock, 1953). A summary of the uncertainties in the data analysis parameters and in the calculated quantities is listed in table 4. Actual values at the experimental points are included in table 3 . The largest contributor to the uncertainties in $\mathrm{T}_{f}, \mathrm{~h}$, and $\mathrm{Nu}$ was the flow distribution uncertainty (that is, whether or not the flow had split evenly into the 12 channels), particularly near the exit of the channels. Due to the approximate nature of determining the channel-to-channel flow distribution (appendix $B$ ), we assume there is still an uncertainty of $\pm 5 \%$ after the adjustment is made.

\section{Results of experiments}

\subsection{Friction factor}

Experiments 1 and 2 (no heating) were conducted to determine the variation of the friction factor with Reynolds number (eq 3 ). Figure 5 shows the variation in $f$ with $R e$ along with a least-squares correlation of the data (for $\operatorname{Re}>5000)$. We have adjusted the flowrate in the channel where the pressure taps were located, according to the method described in appendix $B$. There was no dependence of $f$ on the pressure level. We show the Karman-Nikuradse relation for fully developed turbulent flow in a smooth tube, which according to Hartnett et al. (1962) is applicable to turbulent flow in rectangular channels. We also show the correlation for the results from the tube specimen (Olson and Glover, 1990). These channel specimen data are correlated with

$$
f=0.05058 \cdot R e^{-0.2397}
$$

The standard deviation of the difference between the measured and correlated values is 1.268 . The points for $R e<5000$ were not included in the correlation because the flow was either laminar or transitional.

Our measured values for $f$ were about 27-30\% lower than those of the accepted smooth-tube correlation. The estimated uncertainty in the measured friction factor was 17-18\% for $\operatorname{Re}>4000$. Below $\operatorname{Re}=4000$ the uncertainty increased due to the small pressure drop and large relative uncertainty of the pressure measurement. The are several possible explanations why our data is lower than the accepted correlation. The uncertainty analysis indicates that $f \propto h_{c}{ }^{3}$; hence the uncertainty in $f$ is proportional to 3 times the uncertainty in $h_{c}$. Without sectioning the channel specimen and rendering it unusable for future tests, it was not possible to measure the channel height after the specimen was brazed together. We have assumed an uncertainty in $h_{c}$ of 0.025 $\mathrm{mm}$; if $h_{c}$ was larger by twice the uncertainty, then $f$ would be 27-30\% greater.

Although a greater channel height could explain the discrepancy in $f$, it is more likely $f$ is in error due to the "shortness" of the specimen from a measurement point of view. For the channel, $L / D_{h}=160$, and $4 \mathrm{fL} / \mathrm{D}_{\mathrm{h}} \approx 3$. The dynamic head, $\rho V^{2} / 2$, is 23-36\% of the total pressure drop. If there were a slight burr or protrusion of the pressure tap tube into the channel, the measured pressure could easily be shifted by one or more times the dynamic 
head. This would explain the difference between our measured $f$ and the accepted value. For an accurate measurement of $f$, we would like $4 \mathrm{fL} / \mathrm{D}_{\mathrm{h}} \gg 1$. The uncertainty in $f$ due to the large relative dynamic head was not included in the uncertainty analysis.

Another possible explanation for our discrepancy in $f$ is that we have neglected an entrance length in calculating $f$. Hartnett et al. (1962) show that for turbulent flow in a rectangular channel, the entrance length is about 20 hydraulic diameters. In that region, $f$ is actually less than the fully developed $f$. Including this effect in our data analysis would tend to increase our predicted $f$ in the fully developed region $\left(L / D_{h}>20\right)$.

We used the friction factor correlation developed for the tests without heat transfer to predict the pressure drop when the specimen was heated. Figure 6 compares the error between the predicted pressure drop and the measured pressure drop, plotted as a function of helium flow rate. For low flow rates with $\mathrm{Re}<2000$, the laminar correlation of Rohsenow and Hartnett (1973) was used for $f$. The error is less than 108 for most of the points, and is evenly scattered about the $0 \%$ line. This is within the uncertainty of the measured friction factor.

\subsection{Temperature distributions and heat transfer}

In experiments 3 to 9 we heated the channel specimen to determine the heat transfer performance. A typical plot of temperatures in the helium gas and along the specimen is shown in figure 7. The data are from the lowest flow rate of experiment 8 , which corresponded to the largest inlet-to-outlet temperature rise in the helium. The measured specimen temperatures along the $y$ centerline $(y / W=0.08$ for the hot side, $y / W=-0.04$ for the insulated side), are shown from the inlet to the outlet. The calculated gas temperature is also plotted (eq 12) for the locations of an insulated-side thermocouple. The gas temperature increased approximately linearly from the inlet to the outlet. The heated-side temperatures were $38-44 \mathrm{~K}$ higher than the insulatedside temperatures over most of the specimen. Specimen temperatures on both sides increased steadily from the inlet to the outlet, except that the temperature decreased near the outlet.

The temperature difference between the heated and insulated sides correlates well with the incident normal heat flux. As the heat flux increases, the above temperature difference increases almost linearly. Higher helium flow reduces the temperature difference, because higher flows reduce specimen temperatures and therefore increase the nickel thermal conductivity. Compared to the tube specimen tested previously (Olson and Glover, 1990), this temperature difference was about 4 times greater for the same heat flux. The material thickress between the coolant channel and the heated surface was 3.8 times greater for the channel specimen, which produced the larger temperature difference.

Because the temperature increased from the inlet to the outlet, other fluid properties changed significantly also. Both thermal conductivity and dynamic viscosity increase with temperature, so they increased from the inlet to the outlet. Fluid density decreased from the inlet to the outlet, due primarily 
to the temperature increase but also to the pressure drop. Because density decreased, fluid velocity increased from the inlet to the outlet; for the conditions shown in the figure, the specimen inlet velocity was $18 \mathrm{~m} / \mathrm{s}$ and the specimen outlet velocity was $38 \mathrm{~m} / \mathrm{s}$.

Temperatures at locations perpendicular to the flow direction (y-variation) for the conditions of figure 7 are shown in figure 8 . Here, at each $x-$ location we have plotted temperature on the insulated side as a function of $y$ position. Temperatures were lowest near the middle of the specimen, and highest near both outer edges. The temperature at $\mathrm{x} / \mathrm{L}=0.5$ and $\mathrm{y} / \mathrm{W}=0.44$ was $67 \mathrm{~K}$ higher than the temperature at $\mathrm{x} / \mathrm{L}=0.5$ and $\mathrm{y} / \mathrm{W}=-0.04$. We believe maldistribution of flow (flow in the center channels greater than the flow in the outer channels) was the most likely cause of these variations in temperature. If the flow in a channel were less than the average, the fluid would heat up more as it flowed down the specimen. Also, the lower fluid velocity would produce a smaller heat transfer coefficient, and the wall-tofluid temperature difference would have to be greater to accommodate the heat flux. These two effects would cause higher wall temperatures for regions with flow lower than average; similarly, regions with flow higher than average would have lower wall temperatures.

Table 5 lists the results for the analysis which calculates the channel flow rate based on the measured wall temperatures. For the experiment shown in figure 8, we predict the helium flow in the middle channels is 7.38 greater than the average, while the helium flow in the outer channels is 8.28 less than the average $(y / W=0.363$ to 0.500$)$. The outer channels likely have lower helium flow due to the partial blockage of the channels shown in figure 4. Anomalies in the inlet and outlet manifolds could also cause non-uniform flow. The analysis to determine the flow distribution also indicated that flow maldistribution increased with mass flow, which is consistent with the nonlinearity of pressure drop vs flow rate. The relative helium flow in the middle channel ranged from 3.28 above the average for low flow, experiment 3 , to $11.8 \%$ above average for high flow, experiment 6 . The lowest relative flow was $19.0 \%$ below average for the outer channel in experiment 6 with a high average flow. If we had not used the wall temperature measurements to adjust for the channel flow, the gas temperatures would be calculated incorrectly, which would propagate as an error to both $\mathrm{Re}$ and $\mathrm{Nu}$. For example, for no flow adjustment, in the center channel of the specimen $\mathrm{T}_{f}$ and $\mathrm{Nu}$ would be too high, and $\operatorname{Re}$ would be too low.

In figure 9 we show the heat transfer coefficient and wall-to-fluid temperature difference for the same conditions as those for figure 7 (experiment $8,13.8 \mathrm{~kg} / \mathrm{h}$ helium flow). Shown are points along $\mathrm{y} / \mathrm{W}=-0.04$, from the inlet to the outlet. $h$ was calculated directly from the temperature difference, with the appropriate heat flux (eq 8); to first order the trends in $\mathrm{T}_{\mathrm{w}}-\mathrm{T}_{f}$ and $\mathrm{h}$ are mirrored. The temperature difference increases over the first $60 \%$ of the specimen and decreases over the last 40\%, with the largest percentage change near the furnace end walls ( $x / L=0$ and 1 ). We believe the cause of the drop-off near the end walls was heat conduction through the specimen wall to the inlet and outlet manifolds. The temperatures of the manifolds were the same as the inlet and outlet gas temperatures, which were lower than the specimen wall temperature at $\mathrm{x} / \mathrm{L}=0$ and 1 . We estimated the 
effect of wall conduction using a l-dimensional model of the specimen as a "fin" (Rohsenow and Choi, 1961) assuming: (1) constant heat flux from $0 \leq \mathrm{x} / \mathrm{L}$ $\leq 1$ with zero heat flux for $\mathrm{x} / \mathrm{L}<0$ and $\mathrm{x} / \mathrm{L}>1$; (2) constant fluid temperature for $\mathrm{x} / \mathrm{L}<0$, increasing linearly from $0 \leq \mathrm{x} / \mathrm{L} \leq 1$, and constant for $x / L>1$; and (3) constant heat transfer coefficient. The results showed that in the initial $20 \%$ and final $20 \%$ of the heated zone for this specimen, wall temperatures were significantly influenced by conduction to the manifolds. In these regions, $h$ and therefore $\mathrm{Nu}$ cannot be calculated from eq (8), because the amount of heat convected into the fluid was not the same as that incident on the specimen.

Figure 10 shows the variation of $\mathrm{Re}, \mathrm{Nu}_{\mathrm{m}}$, and $\mathrm{Nu}$ with $\mathrm{x}$ for the same experimental conditions as above. We have plotted $\mathrm{Nu}$ and $\mathrm{Nu}_{\mathrm{m}}$ along the entire heated length, although because of conduction effects the values are accurate only for $0.2<\mathrm{x} / \mathrm{L}<0.8$. The Reynolds number decreased from the inlet to the outlet, due to the increase in viscosity caused by the temperature increase. $\mathrm{Nu}$ and $\mathrm{Nu}_{\mathrm{m}}$ also decreased from the inlet to the outlet, within the $0.2<\mathrm{x} / \mathrm{L}<$ 0.8 region of accuracy. $\mathrm{Nu}_{\mathrm{m}}$ was $14-20 \%$ higher than $\mathrm{Nu}$ for experiment 8.

The trends in temperature distributions, Re, and $\mathrm{Nu}$ with position did not change qualitatively for the other helium flow rates for experiment 8 , nor did they change for the other heat flux rates tested. Figures 7 to 10 are representative of the variations for all runs.

Figure 11 shows the modified Nusselt number plotted against the Reynolds number for all experiments for data points at $\mathrm{y} / \mathrm{W}=-0.04$ and $0.2<\mathrm{x} / \mathrm{L}<0.8$. Also plotted is a correlation from the literature for fully developed turbulent flow in circular tubes (Rohsenow and Hartnett, 1973), along with the correlation for the tube specimen (Olson and Glover, 1990).

$$
\begin{array}{ll}
\text { Rohsenow and Hartnett (1973): } & \mathrm{Nu}=0.022 \cdot \operatorname{Re}^{0.8} \cdot \operatorname{Pr}^{0.6}, \\
\text { Olson and Glover }(1990): & \mathrm{Nu}=0.0420 \cdot \operatorname{Re}^{0.7385} \cdot \operatorname{Pr}^{0.6} .
\end{array}
$$

Rohsenow and Hartnett (1973) recommend using the circular tube correlation for turbulent flow in rectangular channels, with the appropriate hydraulic diameter. The data scatter for past investigators about the correlation curve is often $\pm 30 \%$ (e.g., see Ede, 1961). We have shown the correlation for our data for $\operatorname{Re}>10000$, which is the fully turbulent region. This is

$$
\mathrm{Nu}_{\mathrm{m}}=0.0298 \cdot \mathrm{Re}^{0.7685} \cdot \operatorname{Pr}^{0.6},
$$

or in terms of $\mathrm{Nu}$,

$$
\mathrm{Nu}=0.0298 \cdot \operatorname{Re}^{0.7685} \cdot \operatorname{Pr}^{0.6} \cdot\left(\mathrm{T}_{\mathrm{w}} / \mathrm{T}_{\mathrm{f}}\right)^{-0.55}
$$

The standard deviation between our data and correlation is 2.68 ( $\operatorname{Re}>10000)$. We have assumed a 0.6 power variation on $\mathrm{Pr}$, and the leading coefficient was calculated based on that variation. The Prandtl number was 0.662 to 0.666 . The Reynolds number power and the leading coefficient were calculated from a least-squares fit.

Our data agreed very well with the past correlations, when we accounted for 
the effect of variable properties in the temperature ratio as suggested by Rohsenow and Hartnett (1973). The temperature ratio for these experiments varied from 1.06 to 1.41 , producing a difference between $\mathrm{Nu}$ and $\mathrm{Nu}_{\mathrm{m}}$ of 38 to 218 ( $\mathrm{Nu}_{\mathrm{m}}$ being higher). Without accounting for the effect of variable properties in the temperature ratio, our data fell below the accepted correlations by $20 \%$ at the highest temperature ratio. The uncertainty in the measured $\mathrm{Nu}$ and $\mathrm{Nu}_{\mathrm{m}}$ was 7.68 to $13.8 \%$; the lower uncertainties occurred closer to the inlet, and the higher uncertainties occurred closer to the outlet (due to the contribution from the uncertainty in flow distribution). $\mathrm{Nu}_{\mathrm{m}}$ calculated from the channel specimen correlation is 7.38 lower than $\mathrm{Nu}_{\mathrm{m}}$ calculated from the tube specimen correlation at $\operatorname{Re}=10000$, and 4.38 lower at $\operatorname{Re}=30$ 000. However, data for the tube specimen was not adjusted for the effects of flow maldistribution as was the channel specimen data. We estimate $\mathrm{Nu}$ and $\mathrm{Nu}_{\mathrm{m}}$ for the tube specimen would be about $4 \%$ lower if flow

maldistribution were included. The two correlations therefore agree to within the uncertainty band, and both agree with the accepted smooth-tube correlation within the uncertainty band.

\section{Summary and conclusions}

We have constructed a thin, compact heat exchanger specimen consisting of parallel rectangular channels in a base plate with a cover plate brazed to it. The specimen was made of commercially pure nickel. The specimen was tested in an apparatus which radiatively heated it on one side at a heat flux of up to $77 \mathrm{~W} / \mathrm{cm}^{2}\left(68 \mathrm{Btu} /\left(\mathrm{s} \cdot \mathrm{ft}^{2}\right)\right)$, and cooled the specimen with helium gas at 3.5 to $7.0 \mathrm{MPa}$ (500 to $1000 \mathrm{psi}$ ) and $\mathrm{Re}$ of 1400 to 28000 . Helium gas temperatures ranged from $291 \mathrm{~K}\left(64^{\circ} \mathrm{F}\right)$ to $710 \mathrm{~K}\left(818^{\circ} \mathrm{F}\right)$; the peak specimen temperature was $784 \mathrm{~K}\left(951^{\circ} \mathrm{F}\right)$. Measurements showed the friction factor of the channel specimen was lower than that of a circular tube with fully developed turbulent flow. The discrepancy is attributed to measurement errors arising from the high ratio of dynamic head to frictional pressure drop of the specimen, and to neglecting the entrance region. The measured Nusselt number, when modified to account for the effects of variable properties, agreed with past correlations for fully developed turbulent flow in circular tubes. It also agreed within experimental uncertainty with the Nusselt number for a tube specimen tested earlier. At these temperatures and pressures, there were no unusual effects due to using helium as a heat transfer fluid. Conduction to the end manifolds was important in the first 20\% and last 20\% of the heated portion of the specimen. The flow in the outer channels was as much as $19 \%$ lower than the average flow in the specimen. We anticipate that a specimen which is optimized for heat transfer performance, by making the channels smaller in height and width, would produce a higher pressure drop and also more evenly distributed flow, assuming the brazing operation did not occlude the channels. 


\section{References}

ASME, 1986, "ASME Performance Test Codes Supplement on Instruments and Apparatus - Part 1 - Measurement Uncertainty," ANSI/ASME PTC 19.1-1985.

Ede, A. J., 1961, "The Heat Transfer Coefficient for Flow in a Pipe," Int. J. of Heat Mass Transfer, Vol. 4, pp. 105-110.

Hartnett, J. P., Koh, J. C. Y., and McComas, S. T., 1962, "A Comparison of Predicted and Measured Friction Factors for Turbulent Flow Through Rectangular Ducts," J. of Heat Transfer, Vol. 84, pp. 82-88.

Kline, S. J., and McClintock, F. A., 1953, "Describing Uncertainties in Single-Sample Experiments," Mechanical Engineering, Vol. 75, pp. 3-8.

McCarty, R. D., 1972, "Thermophysical Properties of Helium-4 from 2 to $1500 \mathrm{~K}$ with Pressures to 1000 Atmospheres," NBS-TN-631.

McCarty, R. D., 1973, "Thermodynamic Properties of Helium 4 from 2 to $1500 \mathrm{~K}$ at Pressures to $10^{8} \mathrm{~Pa}, " \mathrm{~J}$. Phys. Chem. Ref. Data, Vol. 2, no. 4, pp. 923 1042 .

Olson, D. A., 1989, "Apparatus for Measuring High-Flux Heat Transfer in Radiatively Heated Compact Exchangers," NISTIR 89-3926.

Olson, D. A., and Glover, M. P., 1990, "Heat Transfer in a Compact Tubular Heat Exchanger With Helium Gas at $3.5 \mathrm{MPa}, "$ NISTIR 3941.

Rohsenow, W. M., and Choi, H., 1961, Heat, Mass, and Momentum Transfer, Prentice-Hall, Inc., Englewood Cliffs.

Rohsenow, W. M., and Hartnett, J. P., 1973, Handbook of Heat Transfer, McGrawHill, Inc., New York.

Scotti, S. J., Martin, C. J., and Lucas, S. H., 1988, "Active Cooling Design for Scramjet Engines Using Optimization Methods," NASA TM-100581.

Shore, C. P., 1986, "Review of Convectively Cooled Structures for Hypersonic Flight," NASA TM-87740. 
A. Heat flux distribution in reflective furnace

A new furnace was installed in the flow apparatus for the experiments on the channel specimen. This furnace had reflective aluminum walls which were water-cooled. The heat flux distribution from the furnace on the target area occupied by the specimen was calibrated using the same procedure described in 0lson (1989). In this method, a calibration specimen was placed in the furnace target area. This specimen was a water-cooled copper plate with 3 heat flow meters $(1.59 \mathrm{~cm}$ by $1.59 \mathrm{~cm})$ soldered to it. With the infrared lamps at steady heating, the calibration specimen with the heat flow meters was traversed over the target area, and the relative heat flow through the meters was measured.

The data were analyzed to convert the measured heat flow to a heat flux function. This function, $f_{q}$, is defined such that when multiplied by the total incident heat flow and divided by the normal area, it gives the local normal heat flux. Or,

$$
\mathrm{q}_{\mathrm{n}}=\mathrm{f}_{\mathrm{q}} \cdot \mathrm{Q}_{\mathrm{T}} / A_{\mathrm{n}} \text {. }
$$

The method for determining $f_{q}$ are described in 01son (1989) and will not be repeated here. The method assumes that the heat flux from the furnace is not dependent on the specimen which is placed in the apparatus.

Calibration experiments were performed at furnace voltage settings of $21.0 \%, 35.5 \%, 50.5 \%, 62.7 \%$, and again at $36.0 \%$ of full scale. Except close to the furnace end walls $(x / L=0$ and $x / L=1)$, the meter heat flow, normalized by the heat flow at $x / L=0.5$, is very similar to the calibration function $f_{q}$. Therefore examining the raw data indicates very accurately the furnace performance. Figure A.l shows the normalized meter heat flow as a function of $\mathrm{x} / \mathrm{L}$ for a furnace voltage of $36 \%$. This is a scan at $\mathrm{y} / \mathrm{W}=0.12 \mathrm{x} / \mathrm{L}$ is the coordinate of the center of the meter. Because of the finite width of the meter, it begins to be shaded by the furnace walls when it is closer than $\mathrm{x} / \mathrm{L}$ $=0.052$ from the furnace walls (shown as the dashed line on the figure).

There are minima in the heat flow near $x / L=0.21$ and $x / L=0.79$. The heat flow reaches maxima at $\mathrm{x} / \mathrm{L}=0.08,0.50$, and 0.92 . The distribution is symmetric about the location $\mathrm{x} / \mathrm{L}=0.5$. This distribution with $\mathrm{x} / \mathrm{L}$ was the same for all heating levels tested. The previous furnace, which had refractory walls, had a symmetric distribution below 298 of full scale voltage, and an asymmetric distribution above 298 of full scale voltage. For the new reflective furnace, there was no variation in heat flux in the $y$ direction.

The uncertainty in the heat flux distribution is \pm 48 ; the major source of uncertainty is the heat flow meter uncertainty. 
B. Method of calculating flow distribution in specimen

For experiments where the specimen is heated, we found wall temperatures to vary in the $y$-direction, perpendicular to the helium flow. If the heat flux were constant over the specimen and the flow in each channel were the same, the temperatures should not vary in the $y$ direction. Since we know from the furnace calibration that heat flux is not a function of $y$, it is most likely that the flow varies from channel to channel. The $x$-ray (fig. 4) of the assembled specimen shows regions of partial flow blockage which could contribute to the non-uniform flow. To calculate fluid temperatures we need to know the flow per channel, and assuming uniform flow will produce errors in $\mathrm{Re}$ and $\mathrm{Nu}$. It is likely that the non-uniform channel flow also exists when the specimen is unheated, so assuming uniform flow will also produce an error in $f$.

We calculate a channel flow distribution by assuming the wall temperature variations in the $\mathrm{y}$ direction are due solely to variations in flow per channel. We neglect solid conduction in the y-direction, which is less than $3 \%$ of the incident heat flux in the worst case. We use the wall temperature measurements at $\mathrm{x} / \mathrm{L}=0.5$, because these probes extend furthest in the + and $y$ directions; there were 5 probes at this location. The identity for the wall temperature at $\mathrm{x}$ and $\mathrm{y}$ is

$$
T_{w x, y}=T_{0}+\left(T_{f x, y}-T_{0}\right)+\left(T_{w x, y}-T_{f x, y}\right) .
$$

The first term in parentheses on the right hand side is the temperature difference due to enthalpy rise of the fluid, while the second term is the temperature difference due to local heat transfer from the solid to the fluid.

We now define an "average" fluid temperature as the temperature of the fluid if the helium flow were equal in each channel. The actual channel fluid temperature and helium flow can be related to the average through the equation

$$
\dot{\mathrm{m}}_{\mathrm{c}} \mathrm{c}_{\mathrm{p}}\left(\mathrm{T}_{\mathrm{fx}, \mathrm{y}}-\mathrm{T}_{0}\right)=(\dot{\mathrm{m}} / \mathrm{n}) \mathrm{c}_{\mathrm{p}}\left(\mathrm{T}_{\mathrm{fx}, \mathrm{ave}}-\mathrm{T}_{0}\right) .
$$

Each side of the equation is obtained by setting it equal to the heat flux added by the furnace up to $x$. We have canceled out the kinetic energy term and the term due to deviations from the non-ideal gas, as both are small and insignificant in making the flow rate adjustment. Rearranging we get

$$
\left(\mathrm{T}_{\mathrm{fx}, \mathrm{y}}-\mathrm{T}_{0}\right)=(\dot{\mathrm{m}} / \mathrm{n}) /\left(\dot{\mathrm{m}}_{\mathrm{c}}\right) \cdot\left(\mathrm{T}_{\mathrm{fx}, \mathrm{ave}}-\mathrm{T}_{0}\right) .
$$

To find the wall-to-fluid temperature difference, we write the local heat transfer equation

$$
T_{w x, y}-T_{a w x, y}=q_{w} / h .
$$

We define an "average" wall temperature as that temperature the wall would attain if the flow were uniform:

$$
\mathrm{T}_{w x} \text {, ave }-\mathrm{T}_{\mathrm{awx}, \text { ave }}=\mathrm{q}_{w} / \mathrm{h}_{\text {ave }} \text {. }
$$


$h_{\text {ave }}$ is also the heat transfer coefficient for uniform flow. Because the heat flux does not depend on the channel flow, B.4 and B.5 can be combined:

$$
T_{w x, y}-T_{f x, y}=\left(T_{w x, a v e}-T_{f x, a v e}\right) \cdot\left(h_{a v e} / h\right) \text {. }
$$

Here we have neglected the kinetic energy term in the adiabatic wall temperature, as variations in it due to flow non-uniformity are not significant.

The heat transfer coefficient, $h$, can be related to the Nusselt number, which is correlated with the Reynolds number and Prandtl number, by

$$
\mathrm{h}=\mathrm{k} / \mathrm{D}_{\mathrm{h}} \cdot \mathrm{Nu}=\mathrm{k} / \mathrm{D}_{\mathrm{h}} \cdot \mathrm{c} \cdot \mathrm{Re}^{\mathrm{a}} \cdot \mathrm{Pr}^{\mathrm{b}}
$$

The ratio of heat transfer coefficients is

$$
\mathrm{h}_{\mathrm{ave}} / \mathrm{h}=\left(\mathrm{k}_{\mathrm{ave}} / \mathrm{k}\right) \cdot\left[(\dot{\mathrm{m}} / \mathrm{n}) /\left(\dot{\mathrm{m}}_{\mathrm{c}}\right)\right]^{\mathrm{a}} \cdot\left(\mu / \mu_{\mathrm{ave}}\right)^{\mathrm{a}} .
$$

We have expressed $R e$ in terms of the flow rate and viscosity. There are no variations in $\operatorname{Pr}$ with temperature, so it cancels. The geometry $\left(D_{h}\right.$ and $\left.A_{f}\right)$ cancels, since it does not depend on the flow distribution. Note also that the leading coefficient on the $\mathrm{Nu}$ vs Re correlation cancels; we do not need to know the magnitude of $\mathrm{Nu}$ for this correction, only the exponent of variation with $\operatorname{Re}$.

We now assume $a=1$. For turbulent flow in a tube, $a \approx 0.8$. Because $\mu$ increases with temperature, $\mu$ increases if $\dot{m}_{c}$ is less than $\dot{m} / n$, and including the viscosity ratio in the flow ratio effectively makes a $>0.8 . \mathrm{k}$ also increases with temperature, so including it with the flow ratio would decrease a again. Letting $a=1$ greatly simplifies the mathematics of the calculation. The error introduced in the adjusted $\mathrm{Nu}$ by using $\mathrm{a}=1.0$ instead of $\mathrm{a}=0.8$ is less than 18. Setting $a=1$ and including the $k$ and $\mu$ ratios in with the flow ratio, we find that

$$
\mathrm{T}_{w x, y}-\mathrm{T}_{f x, y}=\left(\mathrm{T}_{w x, a v e}-\mathrm{T}_{f x, a v e}\right) \cdot(\dot{\mathrm{m}} / \mathrm{n}) /\left(\dot{\mathrm{m}}_{\mathrm{c}}\right)
$$

Substituting eqs (B.9) and (B.3) into (B.I) and simplifying, we get

$$
\dot{\mathrm{m}}_{\mathrm{c}}=(\dot{\mathrm{m}} / \mathrm{n}) \cdot\left(\mathrm{T}_{\mathrm{wx}, \mathrm{ave}}-\mathrm{T}_{0}\right) /\left(\mathrm{T}_{\mathrm{wx}, \mathrm{y}}-\mathrm{T}_{0}\right) \text {. }
$$

The unknowns in this equation are $\mathrm{T}_{w x}$, ave and $\dot{\mathrm{m}}_{\mathrm{c}}$. We measured the total helium flow rate, wall temperatures at $\mathrm{x} / \mathrm{L}=0.5$, and helium inlet temperature. A second equation comes from integrating eq (B.10) across the specimen in the $y$ direction, as the sum of all flows in the individual channels must equal the total measured flow. Or, 


$$
W=\left(T_{w x, a v e}-T_{0}\right) \int_{-W / 2}^{+W / 2} \frac{d y}{\left(T_{w x, y}-T_{0}\right)} .
$$

The integral is approximated as a sum, using the wall temperatures measured at 5 different $\mathrm{y} / \mathrm{W}$ locations for $\mathrm{x} / \mathrm{L}=0.5$. Equation (B.11) is then solved for $\mathrm{T}_{\mathrm{wx} \text {, ave }}$ at $\mathrm{x} / \mathrm{L}=0.5$. The helium flows in the channels are then solved from eq (B.10). Because the wall temperatures are known only at five points, we split the specimen into five regions, with the boundaries between regions at the midpoints between the locations of the 5 temperature probes. Within each region, the flow per channel is considered to be the same.

Since flow from one channel could not penetrate into another channel, the flow per channel (calculated for $\mathrm{x} / \mathrm{L}=0.5$ ) is constant from $\mathrm{x} / \mathrm{L}=0$ to $\mathrm{x} / \mathrm{L}=$ 1. We performed the distribution calculations for each flow rate at each heat flux setting (except for zero heat flux). Table 5 lists the results. For experiment 8, the flow distribution is plotted vs $\mathrm{y} / \mathrm{W}$ in figure B.1. Lines connect the points where the wall temperature is measured and the channel flow is calculated; these are drawn for ease in seeing the trends of flow vs $\mathrm{y} / \mathrm{W}$ and do not imply continuous variation. The channel flow peaks near the center of the specimen and is lower at the outer edges. In addition, the maldistribution accentuates as the total flow increases. These trends were observed for all the heat flux levels tested.

We found the flow distribution was not a function of the level of heat flux. It is likely the same distribution was present when the heat flux was zero, although the wall temperatures were uniform and could not be used to calculate the distribution. To measure the friction factor, we need the flow in the channel near the middle of specimen (where the pressure taps are located), which exceeds $\dot{m} / n$. We correlated $\dot{m}_{c} /(\dot{m} / n)$ in the center channel vs $\dot{m}$ using the results from experiments 3-9, then used that correlation to find $\dot{m}_{c}$ for the friction factor experiments. The correlation had a standard deviation of $0.45 \%$. 
Table 1. Uncertainties in experimental measurements and gas properties at a $95 \%$ confidence interval

\begin{tabular}{|c|c|c|c|}
\hline $\begin{array}{l}\text { Measurement or } \\
\text { Property }\end{array}$ & Technique & $\begin{array}{l}\text { Major Source } \\
\text { of Uncertainty }\end{array}$ & $\begin{array}{l}\text { Magnitude of } \\
\text { Uncertainty }\end{array}$ \\
\hline Gas Flow Rate & $\begin{array}{l}\text { Thermal Mass } \\
\text { Flow Meter }\end{array}$ & Meter Calibration & \pm 18 \\
\hline Heat Flux & $\begin{array}{l}\text { Calibration of } \\
\text { Furnace }\end{array}$ & Heat Flow Meter & \pm 48 \\
\hline $\begin{array}{l}\text { Gas Inlet and } \\
\text { Outlet Temperatures }\end{array}$ & $\begin{array}{l}\text { Platinum Resis- } \\
\text { tance Thermometer }\end{array}$ & Radiation & $\pm 0.5 \mathrm{~K}$ \\
\hline Gas Pressure & $\begin{array}{l}\text { Pressure Trans - } \\
\text { ducer }\end{array}$ & Calibration & \pm 0.258 \\
\hline $\begin{array}{l}\text { Gas Differential } \\
\text { Pressure }\end{array}$ & $\begin{array}{l}\text { Pressure Trans- } \\
\text { ducer }\end{array}$ & Calibration & $\begin{array}{l}\text { greater of } \\
\pm 0.5 \% \text { or } \\
\pm 137 \mathrm{~Pa}\end{array}$ \\
\hline $\begin{array}{l}\text { Specimen } \\
\text { Temperature }\end{array}$ & $\begin{array}{l}\text { Type-N Thermo- } \\
\text { couple }\end{array}$ & $\begin{array}{l}\text { Wire Calibration, } \\
\text { Installation }\end{array}$ & $\begin{array}{l}\text { greater of } \\
\pm 0.4 \% \text { of } \mathrm{T}(\mathrm{C}) \\
\text { or } \pm 1.1 \mathrm{~K}\end{array}$ \\
\hline Gas Density & $\begin{array}{l}\text { Thermodynamic } \\
\text { Function }\end{array}$ & Function Accuracy & $\pm 0.1 \%$ \\
\hline Gas Enthalpy & $\begin{array}{l}\text { Thermodynamic } \\
\text { Function }\end{array}$ & Function Accuracy & \pm 0.28 \\
\hline Gas Specific Heat & $\begin{array}{l}\text { Thermodynamic } \\
\text { Function }\end{array}$ & Function Accuracy & \pm 5 웅 \\
\hline Gas Viscosity & $\begin{array}{l}\text { Thermodynamic } \\
\text { Function }\end{array}$ & Function Accuracy & $\pm 10 \%$ \\
\hline $\begin{array}{l}\text { Gas Thermal } \\
\text { Conductivity }\end{array}$ & $\begin{array}{l}\text { Thermodynamic } \\
\text { Function }\end{array}$ & Function Accuracy & \pm 38 \\
\hline
\end{tabular}


Table 2. Summary of geometrical parameters and experimental conditions for channel specimen

Number of Channels, $\mathrm{n}=20$

Channel Height, $h_{c}=0.559 \mathrm{~mm}$

Channel Width, $\mathrm{w}_{\mathrm{c}}=3.175 \mathrm{~mm}$

Channel Hydraulic Diameter, $D_{h}=0.9503 \mathrm{~mm}$

Specimen Heated Length, $\mathrm{L}=15.24 \mathrm{~cm}$

Specimen Width, $W=7.86 \mathrm{~cm}$

Specimen Heated Normal Area, $A_{n}=119.2 \mathrm{~cm}^{2}$

Specimen Wetted Wall Area, $A_{w}=136.6 \mathrm{~cm}^{2}$

Flow Normal Area, $A_{f}=0.2129 \mathrm{~cm}^{2}$

\begin{tabular}{|c|c|c|c|c|c|c|}
\hline $\begin{array}{c}\text { Expt. } \\
\#\end{array}$ & Date & $\begin{array}{c}\text { Inlet } \\
\text { Pressure } \\
(\mathrm{kPa})\end{array}$ & $\begin{array}{c}\text { Heater } \\
\text { Voltage } \\
(8)\end{array}$ & $\begin{array}{c}\text { Normal } \\
\text { Heat Flux } \\
\left(\mathrm{W} / \mathrm{cm}^{2}\right)\end{array}$ & $\begin{array}{c}\text { Helium } \\
\text { Flow Rate } \\
(\mathrm{kg} / \mathrm{h})\end{array}$ & $\begin{array}{c}\text { Reynolds } \\
\text { Number }\end{array}$ \\
\hline \hline 1 & $7 / 23 / 90$ & 3560 & 0.0 & 0.0 & $2.8-40.3$ & $1800-28000$ \\
2 & $8 / 06 / 90$ & 7250 & 0.0 & 0.0 & $2.4-39.9$ & $1500-27500$ \\
3 & $8 / 06 / 90$ & 3450 & 26.1 & 21.3 & $4.1-39.8$ & $1500-28000$ \\
4 & $7 / 20 / 90$ & 3580 & 50.0 & 42.6 & $10.2-40.0$ & $4000-28000$ \\
5 & $8 / 08 / 90$ & 3545 & 75.4 & 60.9 & $13.3-40.6$ & $5000-28000$ \\
6 & $8 / 10 / 90$ & 6950 & 25.3 & 20.3 & $3.8-41.0$ & $1400-28000$ \\
7 & $8 / 09 / 90$ & 6960 & 50.8 & 42.2 & $9.3-40.9$ & $3500-28000$ \\
8 & $8 / 13 / 90$ & 7000 & 76.3 & 63.9 & $13.8-40.9$ & $5100-28000$ \\
9 & $8 / 14 / 90$ & 7010 & 94.3 & 77.3 & 17.9 & $6800-12000$ \\
\hline
\end{tabular}




\section{Table 3. Data tables for all experiments}

Channel Specimen

Experiment i

Date: 23 July 1990

Time: 09:51:03

$\begin{array}{cccccccc}\text { TA } & T B & M & P O & P O-P 1 & V f & f & \text { Wf } \\ K & K & \mathrm{~kg} / \mathrm{h} & \mathrm{kPa} & \mathrm{kPa} & \boldsymbol{\psi} & & \% \\ 292.73 & 292.28 & 2.81 & 3581.4 & 0.75 & 0.00 & 0.00936 & 25.04\end{array}$

Hot-side Temperatures:

$\begin{array}{ccc}X & Y & T_{W} \\ \mathrm{~cm} & \mathrm{~cm} & K \\ 1.588 & 0.655 & 292.53 \\ 2.540 & 0.655 & 292.53 \\ 5.080 & 0.655 & 292.53 \\ 7.620 & 0.655 & 292.50 \\ 10.160 & 0.655 & 292.49 \\ 12.700 & 0.655 & 292.46 \\ 13.653 & 0.655 & 292.48\end{array}$

Insulated-Side Temperatures and Calculated Data:

\begin{tabular}{|c|c|c|c|c|c|c|c|c|c|c|}
\hline & & & & & & & & 0 & Cal & s. \\
\hline$x$ & $Y$ & $T_{H}$ & Tf & $p$ & v & RE & PR & Htw & Wtf & Wre \\
\hline $\mathrm{cm}$ & $\mathrm{cm}$ & $k$ & $k$ & $\mathrm{KPa}$ & $\mathrm{m} / \mathrm{s}$ & & & K & K & $x$ \\
\hline 0.000 & -0.965 & 92.64 & 292.73 & 3581.4 & 6.57 & 1832 & 0.665 & 1.10 & 0.50 & 1.2 \\
\hline 1.270 & -0.318 & 292.61 & 292.69 & 3581.4 & 6.57 & 1833 & 0.665 & 1.10 & 0.50 & 1.2 \\
\hline 2.527 & -0.330 & 292.57 & 292.65 & 3581.3 & 6.57 & 1833 & 0.665 & 1.10 & 0.50 & 1.27 \\
\hline 3.810 & -0.318 & 292.57 & 292.62 & 3581 & 6.57 & 1833 & 0.665 & 1.10 & 0.50 & 1.27 \\
\hline 5.080 & -0.318 & 292.57 & 292.58 & 358 & .57 & 1833 & 0.665 & 10 & 0.50 & 1.27 \\
\hline 6.363 & -0.318 & 292.53 & 292.55 & 35 & 7 & 1833 & 0.665 & 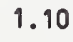 & 0.50 & 1.27 \\
\hline 7.620 & -0.330 & 92.54 & 292.51 & 358 & 6.57 & 1833 & 0.665 & 1.10 & 0.50 & 1.27 \\
\hline 8.903 & -0.305 & 292.53 & 292.47 & 35 & 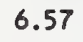 & 1833 & 0.665 & 110 & 0 & .27 \\
\hline .160 & -0.318 & 92.55 & 292.44 & 3580.9 & 6.56 & 1834 & .6 & & 0.50 & 1.27 \\
\hline 430 & 18 & 92 & 292.40 & 3580.9 & .5 & 1834 & .6 & & 0.5 & 1.27 \\
\hline .700 & -0.305 & 292.51 & 292.36 & 35 & 1. & 1834 & 0.6 & 1. & 0. & .27 \\
\hline 3.970 & -0.318 & 292.49 & 292.33 & 35 & 6 & 1834 & 0.6 & 1. & 0. & 1.27 \\
\hline 15.240 & -0.953 & 292.44 & 292.29 & 358 & 6.5 & 1834 & 0.664 & 1. & 0. & 1.27 \\
\hline 2.527 & -2.223 & 292.61 & 292.65 & 3581 & 6.57 & 1833 & 0.665 & 1. & 0.50 & 1.27 \\
\hline 5.080 & -2.235 & 292.51 & 292.58 & 3581 & 6.57 & 1833 & 0.665 & 1. & 0.50 & 1.27 \\
\hline 7.607 & -2.235 & 292.55 & 292.51 & 3581.1 & 6.57 & 1833 & 0.665 & 1. & 0.50 & 11.27 \\
\hline 10.173 & -2.223 & 292.56 & 292.44 & 3580.9 & 6.56 & 1834 & 0.665 & 1. & 0.50 & 1.27 \\
\hline 12.700 & -2.223 & 292.52 & 292.36 & 3580.8 & 6.56 & 1834 & 0.665 & 1.10 & 0.50 & 11.27 \\
\hline 2.540 & 2.197 & 292.57 & 292.65 & 3581.3 & 6.57 & 1833 & 0.665 & 1.10 & 0.50 & 11.27 \\
\hline 5.080 & 2.223 & 292.56 & 292.58 & 3581.2 & 6.57 & 1833 & 0.665 & 1.10 & 0.50 & 11.27 \\
\hline 7.620 & 2.223 & 292.53 & 292.51 & 3581. & 6.57 & 1833 & 0.665 & 1.10 & 0.50 & 1.27 \\
\hline 0.147 & 2.223 & 292.54 & 292.44 & 3580.9 & 6.56 & 1834 & 0.665 & 1.10 & 0.50 & 1.27 \\
\hline 12.713 & 223 & 292.52 & 292.36 & 3580.8 & 6.56 & 1834 & 0.664 & 1.10 & 0.50 & 1.27 \\
\hline 7.607 & -3.493 & 292.58 & 292.51 & 35 & 6.57 & 1833 & 0.665 & 1.10 & 0.50 & 11.27 \\
\hline 7.633 & 3.493 & 292.53 & 292.51 & 3581.1 & 6.57 & 1833 & 0.665 & 1.10 & 0.50 & 11.27 \\
\hline
\end{tabular}


Channel Specimen

Experiment 1

Date: 23 July 1990

Time: 09:55:51

$\begin{array}{cccccccc}T A & T B & M & P 0 & P 0-P 1 & V f & f & \text { Wf } \\ K & K & k g / h & k P a & k P a & \% & & \% \\ 293.26 & 292.88 & 6.07 & 3576.2 & 2.61 & 0.00 & 0.00683 & 17.90\end{array}$

Hot-side Temperatures:

$\begin{array}{ccc}X & Y & T_{H} \\ \mathrm{~cm} & \mathrm{~cm} & K \\ 1.588 & 0.655 & 293.11 \\ 2.540 & 0.655 & 293.11 \\ 5.080 & 0.655 & 293.12 \\ 7.620 & 0.655 & 293.10 \\ 10.160 & 0.655 & 293.11 \\ 12.700 & 0.655 & 293.09 \\ 13.653 & 0.655 & 293.08\end{array}$

Insulated-Side Temperatures and Calculated Data:

\begin{tabular}{|c|c|c|c|c|c|c|c|c|c|c|}
\hline & & & & & & & & \multicolumn{3}{|c|}{ 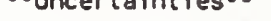 } \\
\hline$x$ & Y & Iw & If & $P$ & $v$ & RE & PR & Htw & Wtf & Hre \\
\hline $\mathrm{cm}$ & $\mathrm{cm}$ & $K$ & $k$ & $\mathrm{kPa}$ & $\mathrm{m} / \mathrm{s}$ & & & $x$ & $K$ & $x$ \\
\hline 0.000 & -0.965 & 293.25 & 293.24 & 3576.2 & 14.39 & 3995 & 0.665 & 1.10 & 0.50 & 11.27 \\
\hline 1.270 & -0.318 & 293.19 & 293.21 & 3575.9 & 14.39 & 3996 & 0.665 & 1.10 & 0.50 & 11.27 \\
\hline 2.527 & -0.330 & 293.20 & 293.18 & 3575.7 & 14.39 & 3996 & 0.665 & 1.10 & 0.50 & 11.27 \\
\hline 3.810 & $\cdot 0.318$ & 293.18 & 293.15 & 3575.5 & 14.38 & 3996 & 0.665 & 1.10 & 0.50 & 11.27 \\
\hline 5.080 & -0.318 & 293.17 & 293.12 & 3575.3 & 14.38 & 3996 & 0.665 & 1.10 & 0.50 & 11.27 \\
\hline 6.363 & -0.318 & 293.16 & 293.09 & 3575.1 & 14.38 & 3997 & 0.665 & 1.10 & 0.50 & 11.27 \\
\hline 7.620 & -0.330 & 293.17 & 293.06 & 3574.9 & 14.38 & 3997 & 0.665 & 1.10 & 0.50 & 11.27 \\
\hline 8.903 & -0.305 & 293.17 & 293.03 & 3574.6 & 14.38 & 3997 & 0.665 & 1.10 & 0.50 & 11.27 \\
\hline 10.160 & -0.318 & 293.17 & 293.00 & 3574.4 & 14.38 & 3998 & 0.665 & 1.10 & 0.50 & 11.27 \\
\hline 11.430 & -0.318 & 293.15 & 292.97 & 3574.2 & 14.38 & 3998 & 0.665 & 1.10 & 0.50 & 11.27 \\
\hline 12.700 & -0.305 & 293.15 & 292.94 & 3574.0 & 14.38 & 3998 & 0.665 & 1.10 & 0.50 & 11.27 \\
\hline 13.970 & -0.318 & 293.14 & 292.91 & 3573.8 & 14.38 & 3998 & 0.665 & 1.10 & 0.50 & 11.27 \\
\hline 15.240 & -0.953 & 293.13 & 292.88 & 3573.6 & 14.38 & 3999 & 0.665 & 1.10 & 0.50 & 11.27 \\
\hline 2.527 & -2.223 & 293.24 & 293.18 & 3575.7 & 14.39 & 3996 & 0.665 & 1.10 & 0.50 & 11.27 \\
\hline 5.080 & -2.235 & 293.09 & 293.12 & 3575.3 & 14.38 & 3996 & 0.665 & 1.10 & 0.50 & 11.27 \\
\hline 7.607 & -2.235 & 293.19 & 293.06 & 3574.9 & 14.38 & 3997 & 0.665 & 1.10 & 0.50 & 11.27 \\
\hline 10.173 & -2.223 & 293.20 & 293.00 & 3574.4 & 14.38 & 3998 & 0.665 & 1.10 & 0.50 & 11.27 \\
\hline 12.700 & -2.223 & 293.19 & 292.94 & 3574.0 & 14.38 & 3998 & 0.665 & 1.10 & 0.50 & 11.27 \\
\hline 2.540 & 2.197 & 293.15 & 293.18 & 3575.7 & 14.39 & 3996 & 0.665 & 1.10 & 0.50 & 11.27 \\
\hline 5.080 & 2.223 & 293.18 & 293.12 & 3575.3 & 14.38 & 3996 & 0.665 & 1.10 & 0.50 & 11.27 \\
\hline 7.620 & 2.223 & 293.14 & 293.06 & 3574.9 & 14.38 & 3997 & 0.665 & 1.10 & 0.50 & 11.27 \\
\hline 10.147 & 2.223 & 293.15 & 293.00 & 3574.4 & 14.38 & 3998 & 0.665 & 1.10 & 0.50 & 11.27 \\
\hline 12.713 & 2.223 & 293.12 & 292.94 & 3574.0 & 14.38 & 3998 & 0.665 & 1.10 & 0.50 & 11.27 \\
\hline 7.607 & -3.493 & 293.23 & 293.06 & 3574.9 & 14.38 & 3997 & 0.665 & 1.10 & 0.50 & 11.27 \\
\hline 7.633 & 3.493 & 293.14 & 293.06 & 3574.9 & 14.38 & 3997 & 0.665 & 1.10 & 0.50 & 11.27 \\
\hline
\end{tabular}




\section{Table 3 (continued)}

Channel Specimen

Experiment 1

Date: 23 July 1990

Time: 10:00:46

$\begin{array}{cccccccc}T A & T B & M & P O & P O-P 1 & V f & f & \text { Wf } \\ K & K & k g / h & K P a & k P a & \% & & \% \\ 293.60 & 293.47 & 9.78 & 3572.1 & 6.35 & 0.00 & 0.00623 & 17.25\end{array}$

Hot-side Temperatures:

$\begin{array}{ccc}X & Y & T H \\ \mathrm{~cm} & \mathrm{~cm} & K \\ 1.588 & 0.655 & 293.52 \\ 2.540 & 0.655 & 293.50 \\ 5.080 & 0.655 & 293.50 \\ 7.620 & 0.655 & 293.50 \\ 10.160 & 0.655 & 293.51 \\ 12.700 & 0.655 & 293.50 \\ 13.653 & 0.655 & 293.52\end{array}$

Insulated-Side Temperatures and Calculated Data:

$\begin{array}{rcccccccccc}X & Y & T H & T f & P & V & R E & P R & \text { Htw } & \text { Htf } & \text { Hre } \\ \mathrm{cm} & \mathrm{Cm} & K & K & k P a & \mathrm{~K} / \mathrm{S} & & & K & K & \text { K } \\ 0.000 & -0.965 & 293.58 & 293.54 & 3572.1 & 23.48 & 6502 & 0.665 & 1.10 & 0.50 & 11.27 \\ 1.270 & -0.318 & 293.53 & 293.53 & 3571.5 & 23.48 & 6502 & 0.665 & 1.10 & 0.50 & 11.27 \\ 2.527 & -0.330 & 293.52 & 293.52 & 3571.0 & 23.48 & 6502 & 0.665 & 1.10 & 0.50 & 11.27 \\ 3.810 & -0.318 & 293.51 & 293.51 & 3570.5 & 23.49 & 6502 & 0.665 & 1.10 & 0.50 & 11.27 \\ 5.080 & -0.318 & 293.53 & 293.50 & 3570.0 & 23.49 & 6502 & 0.665 & 1.10 & 0.50 & 11.27 \\ 6.363 & -0.318 & 293.52 & 293.49 & 3569.4 & 23.49 & 6503 & 0.665 & 1.10 & 0.50 & 11.27 \\ 7.620 & -0.330 & 293.53 & 293.48 & 3568.9 & 23.49 & 6503 & 0.665 & 1.10 & 0.50 & 11.27 \\ 8.903 & -0.305 & 293.51 & 293.47 & 3568.4 & 23.50 & 6503 & 0.665 & 1.10 & 0.50 & 11.27 \\ 10.160 & -0.318 & 293.53 & 293.46 & 3567.8 & 23.50 & 6503 & 0.665 & 1.10 & 0.50 & 11.27 \\ 11.430 & -0.318 & 293.52 & 293.45 & 3567.3 & 23.50 & 6503 & 0.665 & 1.10 & 0.50 & 11.27 \\ 12.700 & -0.305 & 293.52 & 293.45 & 3566.8 & 23.50 & 6503 & 0.665 & 1.10 & 0.50 & 11.27 \\ 13.970 & -0.318 & 293.51 & 293.44 & 3566.3 & 23.51 & 6503 & 0.665 & 1.10 & 0.50 & 11.27 \\ 15.240 & -0.953 & 293.52 & 293.43 & 3565.7 & 23.51 & 6504 & 0.665 & 1.10 & 0.50 & 11.27 \\ 2.527 & -2.223 & 293.54 & 293.52 & 3571.0 & 23.48 & 6502 & 0.665 & 1.10 & 0.50 & 11.27 \\ 5.080 & -2.235 & 293.43 & 293.50 & 3570.0 & 23.49 & 6502 & 0.665 & 1.10 & 0.50 & 11.27 \\ 7.607 & -2.235 & 293.51 & 293.48 & 3568.9 & 23.49 & 6503 & 0.665 & 1.10 & 0.50 & 11.27 \\ 10.173 & -2.223 & 293.52 & 293.46 & 3567.8 & 23.50 & 6503 & 0.665 & 1.10 & 0.50 & 11.27 \\ 12.700 & -2.223 & 293.50 & 293.45 & 3566.8 & 23.50 & 6503 & 0.665 & 1.10 & 0.50 & 11.27 \\ 2.540 & 2.197 & 293.47 & 293.52 & 3571.0 & 23.48 & 6502 & 0.665 & 1.10 & 0.50 & 11.27 \\ 5.080 & 2.223 & 293.47 & 293.50 & 3570.0 & 23.49 & 6502 & 0.665 & 1.10 & 0.50 & 11.27 \\ 7.620 & 2.223 & 293.47 & 293.48 & 3568.9 & 23.49 & 6503 & 0.665 & 1.10 & 0.50 & 11.27 \\ 10.147 & 2.223 & 293.46 & 293.46 & 3567.9 & 23.50 & 6503 & 0.665 & 1.10 & 0.50 & 11.27 \\ 12.713 & 2.223 & 293.47 & 293.45 & 3566.8 & 23.50 & 6503 & 0.665 & 1.10 & 0.50 & 11.27 \\ 7.607 & -3.493 & 293.54 & 293.48 & 3568.9 & 23.49 & 6503 & 0.665 & 1.10 & 0.50 & 11.27 \\ 7.633 & 3.493 & 293.46 & 293.48 & 3568.9 & 23.49 & 6503 & 0.665 & 1.10 & 0.50 & 11.27\end{array}$


Table 3 (continued)

Channel Specimen

Experiment 1

Date: 23 July 1990

rime: 10:05:29

$\begin{array}{cccccccc}\text { TA } & T B & M & P O & P 0-P 1 & V f & f & \text { Uf } \\ K & K & \mathrm{~kg} / \mathrm{h} & \mathrm{KPa} & \mathrm{KPa} & \% & & \text { X } \\ 292.76 & 292.94 & 15.39 & 3566.6 & 14.41 & 0.00 & 0.00554 & 17.14\end{array}$

Hot-side Temperatures:

$\begin{array}{ccc}X & Y & \text { TH } \\ \mathrm{cm} & \mathrm{cm} & K \\ 1.588 & 0.655 & 292.72 \\ 2.540 & 0.655 & 292.77 \\ 5.080 & 0.655 & 292.73 \\ 7.620 & 0.655 & 292.77 \\ 10.160 & 0.655 & 292.76 \\ 12.700 & 0.655 & 292.76 \\ 13.653 & 0.655 & 292.76\end{array}$

Insulated-Side Temperatures and Calculated Data:

\begin{tabular}{|c|c|c|c|c|c|c|c|c|c|c|}
\hline & & & & & & & & & & \\
\hline$x$ & $\gamma$ & $I_{H}$ & Tf & $\mathbf{P}$ & $v$ & RE & PR & Wew & Wef & Wre \\
\hline $\mathrm{cm}$ & $\mathrm{cm}$ & $K$ & $k$ & $\mathrm{kPa}$ & $\mathrm{m} / \mathrm{s}$ & & & $K$ & K & $x$ \\
\hline 0.000 & -0.965 & 292.77 & 292.63 & 3566.6 & 37.40 & 10395 & 0.665 & 1.10 & 0.50 & 11.27 \\
\hline 1.270 & -0.318 & 292.71 & 292.64 & 3565.4 & 1 & 10394 & 0.665 & 1.10 & 0.50 & 11.27 \\
\hline 2.527 & -0.330 & 292.71 & 292.66 & 3564.2 & 42 & 10394 & 0.665 & 1.10 & 0.50 & 1.27 \\
\hline 3.810 & -0.318 & 292.74 & 92.67 & 3563 & 37.44 & 10394 & 0.665 & 0 & 0.50 & 1.27 \\
\hline 5.080 & -0.3 & 92.73 & 92.68 & 356 & 5 & 10393 & 65 & 0 & 0 & .27 \\
\hline 363 & -0.3 & 292.72 & 292.70 & 35 & 7 & 10393 & 65 & 0 & 0 & 27 \\
\hline 7.620 & -0.330 & 292.73 & 292.71 & 355 & 8 & 10393 & 0.665 & 0 & 0 & 27 \\
\hline 8.903 & -0.305 & 292.76 & 292.73 & 3558.2 & 37.50 & 10393 & 0.665 & 0 & .50 & .27 \\
\hline 0.160 & -0.318 & 292.71 & 292.74 & 3557.0 & 37.51 & 10392 & 0.665 & .10 & .50 & .27 \\
\hline 11.430 & -0.318 & 292.73 & 292.75 & 3555.8 & 37.53 & 10392 & 0.665 & 1.10 & 0.50 & 11.27 \\
\hline 12.700 & -0.305 & 292.73 & 292.77 & 3554.6 & 37.54 & 10392 & 0.665 & 1.10 & 0.50 & 11.27 \\
\hline 13.970 & -0.318 & 292.75 & 292.78 & 3553.4 & 37.55 & 10391 & 0.665 & 1.10 & 0.50 & 11.27 \\
\hline 15.240 & -0.953 & 292.71 & 292.79 & 3552.2 & 37.57 & 10391 & 0.665 & 1.10 & 0.50 & 11.27 \\
\hline 2.527 & -2.223 & 292.67 & 292.66 & 3564.2 & 37.42 & 10394 & 0.665 & 1.10 & 0.50 & 11.27 \\
\hline 5.080 & -2.235 & 292.75 & 292.68 & 3561.8 & 37.45 & 10393 & 0.665 & 1.10 & 0.50 & 11.27 \\
\hline 7.607 & -2.235 & 292.71 & 292.71 & 3559.4 & 37.48 & 10393 & 0.665 & 1.10 & 0.50 & 11.27 \\
\hline 10.173 & -2.223 & 292.70 & 292.74 & 3557.0 & 37.51 & 10392 & 0.665 & 1.10 & 0.50 & 11.27 \\
\hline 12.700 & -2.223 & 292.67 & 292.77 & 3554.6 & 37.54 & 10392 & 0.665 & 1.10 & 0.50 & 11.27 \\
\hline 2.540 & 2.197 & 292.68 & 292.66 & 3564.2 & 37.43 & 10394 & 0.665 & 1.10 & 0.50 & 11.27 \\
\hline 5.080 & 2.223 & 292.72 & 292.68 & 3561.8 & 37.45 & 10393 & 0.665 & 1.10 & 0.50 & 11.27 \\
\hline 7.620 & 2.223 & 292.67 & 292.71 & 3559.4 & 37.48 & 10393 & 0.665 & 1.10 & 0.50 & 11.27 \\
\hline 10.147 & 2.223 & 292.70 & 292.74 & 3557.0 & 37.51 & 10392 & 0.665 & 1.10 & 0.50 & 11.27 \\
\hline 12.713 & 2.223 & 292.69 & 292.77 & 3554.6 & 37.54 & 10392 & 0.665 & 1.10 & 0.50 & 11.27 \\
\hline 7.607 & -3.493 & 292.65 & 292.71 & 3559.4 & 37.48 & 10393 & 0.665 & 1.10 & 0.50 & 11.27 \\
\hline 7.633 & 3.493 & 292.67 & 292.71 & 3559.4 & 37.48 & 10393 & 0.665 & 1.10 & 0.50 & 11.27 \\
\hline
\end{tabular}


Channel specimen

Experiment 1

Date: 23 July 1990

Time: 10:09:54

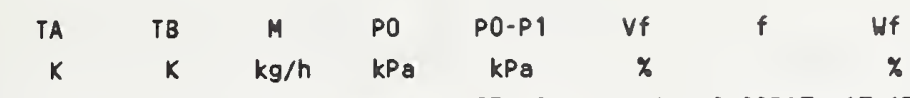

$\begin{array}{llllllll}292.06 & 292.21 & 20.17 & 3561.1 & 23.59 & 0.01 & 0.00517 & 17.13\end{array}$

Hot-side Temperatures:

$\begin{array}{ccc}X & Y & T_{H} \\ \mathrm{~cm} & \mathrm{~cm} & K \\ 1.588 & 0.655 & 292.06 \\ 2.540 & 0.655 & 292.08 \\ 5.080 & 0.655 & 292.12 \\ 7.620 & 0.655 & 292.10 \\ 10.160 & 0.655 & 292.11 \\ 12.700 & 0.655 & 292.11 \\ 13.653 & 0.655 & 292.11\end{array}$

Insulated-Side Temperatures and Calculated Data:

\begin{tabular}{|c|c|c|c|c|c|c|c|c|c|c|}
\hline \multirow[b]{2}{*}{$x$} & \multirow[b]{2}{*}{$Y$} & \multirow[b]{2}{*}{ Tн } & \multirow[b]{2}{*}{$T f$} & \multirow[b]{2}{*}{$\mathbf{P}$} & \multirow[b]{2}{*}{ v } & \multirow[b]{2}{*}{ RE } & \multirow[b]{2}{*}{ PR } & \multicolumn{3}{|c|}{--Uncertainties-- } \\
\hline & & & & & & & & Wew & Wtf & Wre \\
\hline $\mathrm{cm}$ & $\mathrm{cm}$ & K & K & $\mathrm{kPa}$ & $\mathrm{m} / \mathrm{s}$ & & & K & $k$ & $x$ \\
\hline 0.000 & -0.965 & 292.11 & 291.83 & 3561.1 & 49.44 & 13783 & 0.664 & 1.10 & 0.50 & 11.27 \\
\hline 1.270 & -0.318 & 292.08 & 291.84 & 3559.1 & 49.47 & 13783 & 0.664 & 1.10 & 0.50 & 11.27 \\
\hline 2.527 & -0.330 & 292.08 & 291.85 & 3557.2 & 49.50 & 13783 & 0.664 & 1.10 & 0.50 & 11.27 \\
\hline 3.810 & -0.318 & 292.09 & 291.86 & 3555.2 & 49.53 & 13783 & 0.664 & 1.10 & 0.50 & 11.27 \\
\hline 5.080 & -0.318 & 292.10 & 291.88 & 3553.2 & 49.56 & 13782 & 0.664 & 1.10 & 0.50 & 11.27 \\
\hline 6.363 & -0.318 & 292.07 & 291.89 & 3551.2 & 49.59 & 13782 & 0.665 & 1.10 & 0.50 & 11.27 \\
\hline 7.620 & -0.330 & 292.09 & 291.90 & 3549.3 & 49.62 & 13782 & 0.665 & 1.10 & 0.50 & 11.27 \\
\hline 8.903 & -0.305 & 292.10 & 291.91 & 3547.3 & 49.64 & 13781 & 0.665 & 1.10 & 0.50 & 11.27 \\
\hline 10.160 & -0.318 & 292.09 & 291.92 & 3545.4 & 49.67 & 13781 & 0.665 & 1.10 & 0.50 & 11.27 \\
\hline 11.430 & -0.318 & 292.08 & 291.93 & 3543.4 & 49.70 & 13781 & 0.665 & 1.10 & 0.50 & 11.27 \\
\hline 12.700 & -0.305 & 292.09 & 291.94 & 3541.4 & 49.73 & 13780 & 0.665 & 1.10 & 0.50 & 11.27 \\
\hline 13.970 & -0.318 & 292.10 & 291.95 & 3539.5 & 49.76 & 13780 & 0.665 & 1.10 & 0.50 & 11.27 \\
\hline 15.240 & -0.953 & 292.07 & 291.96 & 3537.5 & 49.79 & 13780 & 0.665 & 1.10 & 0.50 & 11.27 \\
\hline 2.527 & -2.223 & 292.08 & 291.85 & 3557.2 & 49.50 & 13783 & 0.664 & 1.10 & 0.50 & 11.27 \\
\hline 5.080 & -2.235 & 292.14 & 291.88 & 3553.2 & 49.56 & 13782 & 0.664 & 1.10 & 0.50 & 11.27 \\
\hline 7.607 & -2.235 & 292.08 & 291.90 & 3549.3 & 49.62 & 13782 & 0.665 & 1.10 & 0.50 & 11.27 \\
\hline 10.173 & -2.223 & 292.08 & 291.92 & 3545.3 & 49.67 & 13781 & 0.665 & 1.10 & 0.50 & 11.27 \\
\hline 12.700 & -2.223 & 292.08 & 291.94 & 3541.4 & 49.73 & 13780 & 0.665 & 1.10 & 0.50 & 11.27 \\
\hline 2.540 & 2.197 & 292.09 & 291.85 & 3557.1 & 49.50 & 13783 & 0.664 & 1.10 & 0.50 & 11.27 \\
\hline 5.080 & 2.223 & 292.10 & 291.88 & 3553.2 & 49.56 & 13782 & 0.664 & 1.10 & 0.50 & 11.27 \\
\hline 7.620 & 2.223 & 292.07 & 291.90 & 3549.3 & 49.62 & 13782 & 0.665 & 1.10 & 0.50 & 11.27 \\
\hline 10.147 & 2.223 & 292.06 & 291.92 & 3545.4 & 49.67 & 13781 & 0.665 & 1.10 & 0.50 & 11.27 \\
\hline 12.713 & 2.223 & 292.11 & 291.94 & 3541.4 & 49.73 & 13780 & 0.665 & 1.10 & 0.50 & 11.27 \\
\hline 7.607 & -3.493 & 292.05 & 291.90 & 3549.3 & 49.62 & 13782 & 0.665 & 1.10 & 0.50 & 11.27 \\
\hline 7.633 & 3.493 & 292.10 & 291.90 & 3549.3 & 49.62 & 13782 & 0.665 & 1.10 & 0.50 & 11.27 \\
\hline
\end{tabular}


Table 3 (continued)

Channel Specimen

Experiment 1

Date: 23 July 1990

Time: $10: 14: 27$

$\begin{array}{cccccccc}\text { TA } & \text { TB } & \text { M } & \text { PO } & \text { PO-P1 } & \text { Vf } & f & \text { Uf } \\ K & K & k g / h & \text { KPa } & \text { KPa } & \text { X } & & \text { \% } \\ 291.81 & 291.92 & 24.92 & 3554.3 & 34.74 & 0.00 & 0.00489 & 17.13\end{array}$

Hot-side Temperatures:

$\begin{array}{ccc}X & Y & T W \\ \mathrm{~cm} & \mathrm{~cm} & K \\ 1.588 & 0.655 & 291.81 \\ 2.540 & 0.655 & 291.79 \\ 5.080 & 0.655 & 291.81 \\ 7.620 & 0.655 & 291.80 \\ 10.160 & 0.655 & 291.81 \\ 12.700 & 0.655 & 291.82 \\ 13.653 & 0.655 & 291.81\end{array}$

Insulated-side Temperatures and Calculated Data:

\begin{tabular}{|c|c|c|c|c|c|c|c|c|c|c|}
\hline & & & & & & & & & 8 & es.- \\
\hline$x$ & $y$ & $T_{W}$ & If & $P$ & v & RE & PR & Wtw & Wtf & Wre \\
\hline $\mathrm{cm}$ & $\mathrm{cm}$ & K & K & $\mathrm{kPa}$ & $\mathrm{m} / \mathrm{s}$ & & & K & K & $x$ \\
\hline 0.000 & -0.965 & 291.86 & 291.45 & 3554.3 & 61.61 & 17180 & 0.664 & 1.10 & 0.50 & 11.27 \\
\hline 1.270 & -0.318 & 291.82 & 291.46 & 3551.4 & 61.66 & 17180 & 0.664 & 1.10 & 0.50 & 11.27 \\
\hline 2.527 & -0.330 & 291.80 & 291.46 & 3548.5 & 61.71 & 17179 & 0.664 & 1.10 & 0.50 & 11.27 \\
\hline 3.810 & -0.318 & 291.82 & 291.47 & 3545.6 & 61.76 & 17179 & 0.664 & 1.10 & 0.50 & 11.27 \\
\hline 5.080 & -0.318 & 291.82 & 291.48 & 3542.7 & 61.81 & 17179 & 0.664 & 1.10 & 0.50 & 11.27 \\
\hline 6.363 & -0.318 & 291.81 & 291.49 & 3539.8 & 61.87 & 17179 & 0.664 & 1.10 & 0.50 & 1.27 \\
\hline 7.620 & -0.330 & 291.81 & 291.49 & 3536.9 & 61.92 & 17179 & 0.664 & 1.10 & 0.50 & 1.27 \\
\hline 8.903 & -0.305 & 291.79 & 291.50 & 3534.0 & 61.97 & 17178 & 0.664 & 1.10 & 0.50 & 1.27 \\
\hline 10.160 & -0.318 & 291.82 & 291.51 & 3531.1 & 62.02 & 17178 & 0.665 & 1.10 & 0. & 11.27 \\
\hline 11.430 & -0.318 & 291.82 & 291.52 & 3528.2 & 62.07 & 17178 & 0.665 & 1.10 & 0.50 & 1.27 \\
\hline 12.700 & -0.305 & 291.83 & 291.53 & 3525.3 & 62.12 & 17178 & 0.665 & 1.10 & 0.50 & 1.27 \\
\hline 13.970 & -0.318 & 291.84 & 291.53 & 3522.4 & .17 & 17178 & 0.6 & 1.10 & 0. & 1.27 \\
\hline 15.240 & -0.953 & 291.82 & 291.54 & 351 & 3 & 17177 & 0.6 & 1.10 & 0 & 1.27 \\
\hline 2.527 & -2. & 291.80 & 291.46 & 354 & 71 & 17179 & 0.6 & 1.10 & 0. & 1.27 \\
\hline 5.080 & -2.235 & 291.87 & 291.48 & 3542 & 81 & 17179 & 0. & 1.10 & 0. & 11.27 \\
\hline 7.607 & -2.235 & 291.84 & 291.49 & 3536.9 & 61.92 & 17179 & 0.664 & 1.10 & 0.50 & 11.27 \\
\hline 10.173 & -2.223 & 291.80 & 291.51 & 3531.1 & 62.02 & 17178 & 0.665 & 1.10 & 0.50 & 11.27 \\
\hline 12.700 & -2.223 & 291.83 & 291.53 & 3525.3 & 62.12 & 17178 & 0.665 & 1.10 & 0.50 & 11.27 \\
\hline 2.540 & 2.197 & 291.83 & 291.46 & 3548.5 & 61.71 & 17179 & 0.664 & 1.10 & 0.50 & 11.27 \\
\hline 5.080 & 2.223 & 291.81 & 291.48 & 3542.7 & 61.81 & 17179 & 0.664 & 1.10 & 0.50 & 11.27 \\
\hline 7.620 & 2.223 & 291.82 & 291.49 & 3536.9 & 61.92 & 17179 & 0.664 & 1.10 & 0.50 & 11.27 \\
\hline 10.147 & 2.223 & 291.83 & 291.51 & 3531.1 & 62.02 & 17178 & 0.665 & 1.10 & 0.50 & 11.27 \\
\hline 12.713 & 2.223 & 291.85 & 291.53 & 3525.3 & 62.12 & 17178 & 0.665 & 1.10 & 0.50 & 11.27 \\
\hline 7.607 & -3.493 & 291.80 & 291.49 & 3536.9 & 61.92 & 17179 & 0.664 & 1.10 & 0.50 & 11.27 \\
\hline 7.633 & 3.493 & 291.87 & 291.49 & 3536.9 & 61.92 & 17179 & 0.664 & 1.10 & 0.50 & 11.27 \\
\hline
\end{tabular}




\section{Table 3 (continued)}

Channel specimen

Experiment 1

Date: 23 July 1990

Time: $10: 18: 44$

$\begin{array}{cccccccc}\text { TA } & T B & M & P O & P O-P 1 & V f & f & \text { Wf } \\ K & K & \mathrm{~kg} / \mathrm{h} & \mathrm{KPg} & \mathrm{kPa} & \% & & \times \\ 291.64 & 201.75 & 30.62 & 3544.0 & 51.14 & 0.00 & 0.00466 & 17.13\end{array}$

$\begin{array}{llllllll}291.64 & 291.75 & 30.62 & 3544.0 & 51.14 & 0.00 & 0.00466 & 17.13\end{array}$

Hot-side Temperatures:

$\begin{array}{ccc}X & Y & T w \\ \mathrm{~cm} & \mathrm{~cm} & K \\ 1.588 & 0.655 & 291.61 \\ 2.540 & 0.655 & 291.63 \\ 5.080 & 0.655 & 291.60 \\ 7.620 & 0.655 & 291.63 \\ 10.160 & 0.655 & 291.63 \\ 12.700 & 0.655 & 291.64 \\ 13.653 & 0.655 & 291.67\end{array}$

Insulated-side Temperatures and Calculated Data:

\begin{tabular}{|c|c|c|c|c|c|c|c|c|c|c|}
\hline \multirow[b]{2}{*}{$x$} & \multirow[b]{2}{*}{ Y } & \multirow[b]{2}{*}{$T w$} & \multirow[b]{2}{*}{ Tf } & \multirow[b]{2}{*}{$\mathbf{P}$} & \multirow[b]{2}{*}{$v$} & \multirow[b]{2}{*}{ RE } & \multirow[b]{2}{*}{ PR } & \multicolumn{3}{|c|}{--Uncertainties.- } \\
\hline & & & & & & & & Wtw & Wtf & Wre \\
\hline $\mathrm{cm}$ & $\mathrm{cm}$ & K & $k$ & $\mathrm{KPa}$ & $\mathrm{m} / \mathrm{s}$ & & & $k$ & K & $x$ \\
\hline 0.000 & -0.965 & 291.68 & 291.08 & 3544.0 & 76.38 & 21282 & 0.664 & 1.10 & 0.51 & 11.27 \\
\hline 1.270 & -0.318 & 291.63 & 291.09 & 3539.7 & 76.47 & 21282 & 0.664 & 1.10 & 0.51 & 11.27 \\
\hline 2.527 & -0.330 & 291.61 & 291.09 & 3535.5 & 76.56 & 21282 & 0.664 & 1.10 & 0.51 & 11.27 \\
\hline 3.810 & -0.318 & 291.66 & 291.10 & 3531.2 & 76.65 & 21282 & 0.664 & 1.10 & 0.51 & 11.27 \\
\hline 5.080 & -0.318 & 291.61 & 291.11 & 3527.0 & 76.75 & 21282 & 0.664 & 1.10 & 0.51 & 11.27 \\
\hline 6.363 & -0.318 & 291.64 & 291.11 & 3522.7 & 76.84 & 21281 & 0.664 & 1.10 & 0.51 & 11.27 \\
\hline 7.620 & -0.330 & 291.65 & 291.12 & 3518.4 & 76.93 & 21281 & 0.664 & 1.10 & 0.51 & 11.27 \\
\hline 8.903 & -0.305 & 291.64 & 291.13 & 3514.1 & 77.03 & 21281 & 0.664 & 1.10 & 0.51 & 11.27 \\
\hline 10.160 & -0.318 & 291.62 & 291.14 & 3509.9 & 77.12 & 21281 & 0.665 & 1.10 & 0.51 & 11.27 \\
\hline 11.430 & -0.318 & 291.63 & 291.14 & 3505.7 & 77.21 & 21281 & 0.665 & 1.10 & 0.51 & 11.27 \\
\hline 12.700 & -0.305 & 291.64 & 291.15 & 3501.4 & 77.31 & 21280 & 0.665 & 1.10 & 0.51 & 11.27 \\
\hline 13.970 & -0.318 & 291.67 & 291.16 & 3497.1 & 77.40 & 21280 & 0.665 & 1.10 & 0.51 & 11.27 \\
\hline 15.240 & -0.953 & 291.64 & 291.16 & 3492.9 & 77.50 & 21280 & 0.665 & 1.10 & 0.51 & 11.27 \\
\hline 2.527 & -2.223 & 291.62 & 291.09 & 3535.5 & 76.56 & 21282 & 0.664 & 1.10 & 0.51 & 11.27 \\
\hline 5.080 & -2.235 & 291.71 & 291.11 & 3527.0 & 76.75 & 21282 & 0.664 & 1.10 & 0.51 & 11.27 \\
\hline 7.607 & -2.235 & 291.65 & 291.12 & 3518.5 & 76.93 & 21281 & 0.664 & 1.10 & 0.51 & 11.27 \\
\hline 10.173 & -2.223 & 291.64 & 291.14 & 3509.9 & $\pi .12$ & 21281 & 0.665 & 1.10 & 0.51 & 11.27 \\
\hline 12.700 & -2.223 & 291.63 & 291.15 & 3501.4 & 77.31 & 21280 & 0.665 & 1.10 & 0.51 & 11.27 \\
\hline 2.540 & 2.197 & 291.65 & 291.09 & 3535.5 & 76.56 & 21282 & 0.664 & 1.10 & 0.51 & 11.27 \\
\hline 5.080 & 2.223 & 291.63 & 291.11 & 3527.0 & 76.75 & 21282 & 0.664 & 1.10 & 0.51 & 11.27 \\
\hline 7.620 & 2.223 & 291.66 & 291.12 & 3518.4 & 76.93 & 21281 & 0.664 & 1.10 & 0.51 & 11.27 \\
\hline 10.147 & 2.223 & 291.64 & 291.14 & 3510.0 & 77.12 & 21281 & 0.665 & 1.10 & 0.51 & 11.27 \\
\hline 12.713 & 2.223 & 291.68 & 291.15 & 3501.3 & 77.31 & 21280 & 0.665 & 1.10 & 0.51 & 11.27 \\
\hline 7.607 & -3.493 & 291.65 & 291.12 & 3518.5 & 76.93 & 21281 & 0.664 & 1.10 & 0.51 & 11.27 \\
\hline 7.633 & 3.493 & 291.65 & 291.12 & 3518.4 & 76.93 & 21281 & 0.664 & 1.10 & 0.51 & 11.27 \\
\hline
\end{tabular}




\section{Table 3 (continued)}

Channel specimen

Experiment 1

Date: 23 July 1990

Time: 10:23:06

$\begin{array}{cccccccc}T A & T B & M & P O & P O-P 1 & V F & f & H f \\ K & K & K g / h & K P a & K P g & \% & & \% \\ 291.46 & 291.61 & 40.31 & 3524.9 & 86.53 & 0.00 & 0.00442 & 17.13\end{array}$

Hot-side Temperatures:

$\begin{array}{ccc}X & Y & T_{H} \\ \mathrm{~cm} & \mathrm{~cm} & K \\ 1.588 & 0.655 & 291.38 \\ 2.540 & 0.655 & 291.41 \\ 5.080 & 0.655 & 291.61 \\ 7.620 & 0.655 & 291.42 \\ 10.160 & 0.655 & 291.38 \\ 12.700 & 0.655 & 291.44 \\ 13.653 & 0.655 & 291.46\end{array}$

Insulated-Side Temperatures and Calculated Dato:

\begin{tabular}{|c|c|c|c|c|c|c|c|c|c|c|}
\hline & & & & & & & & & & \\
\hline 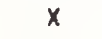 & $Y$ & & If & & v & RE & PR & $w$ & Wtf & Wre \\
\hline $\mathrm{cm}$ & $\mathrm{Cr}$ & 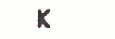 & K & $\mathrm{KPa}$ & $\mathrm{m} / \mathrm{s}$ & & & & K & $x$ \\
\hline 000 & -0.965 & 11.46 & 290.48 & 3524.9 & 101.57 & 28248 & 0.664 & 1.10 & 0.52 & 1.27 \\
\hline 1.270 & -0.318 & 81.40 & 290.48 & 3517.7 & 101.77 & 28248 & 0.664 & 1.10 & 0.52 & 1.27 \\
\hline 2.527 & -0.330 & 91.42 & 290.49 & 3510.6 & 101.98 & 28248 & 0.664 & 1.10 & 0.52 & 11.27 \\
\hline .810 & -0.318 & 291.41 & 290.50 & 3503.3 & 102.19 & 28248 & 0.664 & 9.10 & 0.52 & 11.27 \\
\hline .080 & -0.318 & 91.41 & 290.51 & 3496.1 & 102.40 & 28248 & 0.664 & 1.10 & .52 & 11.27 \\
\hline 6.363 & -0.318 & 291.42 & 290.51 & 3488.8 & 102.61 & 28248 & 0.664 & .10 & 0.52 & 11.27 \\
\hline 7.620 & -0.330 & 291.42 & 290.52 & 3481.7 & 102.82 & 28248 & 0.664 & 1.10 & 0.52 & 19.27 \\
\hline 8.903 & -0.305 & 291.44 & 290.52 & 347 & 103 & 28248 & 0.665 & 1.10 & .52 & 11.27 \\
\hline .160 & -0.318 & 39 & 290.53 & 3467.2 & 103.25 & 28248 & 0.6 & .10 & .52 & 11.27 \\
\hline .630 & -0.318 & 291.43 & 290.54 & 3460.0 & 103.46 & 28248 & 0.665 & .10 & .52 & 11.27 \\
\hline .700 & -0.305 & 291.43 & 290.54 & 3452.8 & 103.67 & 28248 & 0.665 & 1.10 & .52 & 11.27 \\
\hline 3.970 & -0.318 & 291.45 & 290.55 & 3445.6 & 103 & 28248 & 0.6 & 1.10 & .52 & 11.27 \\
\hline 15.260 & -0.953 & 291.45 & 290.56 & 3438.4 & 10 & 28248 & 0.6 & 1.10 & .52 & 11.27 \\
\hline 2.527 & -2.223 & 291.43 & 290.49 & 3510.6 & 101.98 & 28248 & 0.66 & 1.10 & .52 & 11.27 \\
\hline 5.080 & -2.235 & 291.67 & 290.51 & 3496.1 & 102.40 & 28248 & 0.664 & 1.10 & .52 & 11.27 \\
\hline 7.607 & -2.235 & 291.44 & 290.52 & 3481.7 & 10 & 28248 & 0.664 & 1.10 & .52 & 11.27 \\
\hline 10.173 & -2.223 & 291.63 & 290.53 & 3467.2 & 103.25 & 28248 & 0.665 & 1.10 & 0.52 & 11.27 \\
\hline 12.700 & $\cdot 2.223$ & 291.44 & 290.54 & 3452.8 & 103.67 & 28248 & 0.665 & 1.10 & 0.52 & 11.27 \\
\hline 2.540 & 2.197 & 291.43 & 290.49 & 3510.5 & 101.98 & 28248 & 0.664 & 1.10 & 0.52 & 11.27 \\
\hline 5.080 & 2.223 & 291.43 & 290.51 & 3496.1 & 102.40 & 28248 & 0.664 & 1.10 & 0.52 & 11.27 \\
\hline 7.620 & 2.223 & 291.41 & 290.52 & 3481.7 & 102.82 & 28248 & 0.664 & 1.10 & 0.52 & 11.27 \\
\hline 10.147 & 2.223 & 291.41 & 290.53 & 3467.3 & 103.24 & 28248 & 0.665 & 1.10 & 0.52 & 11.27 \\
\hline 12.713 & 223 & 91.43 & 290.54 & 3452.7 & 103.68 & 28248 & 0.665 & 1.10 & 0.52 & 11.27 \\
\hline 7.607 & $\cdot 3.493$ & 291.43 & 290.52 & 3481.7 & 102.82 & 28248 & 0.664 & 1.10 & 0.52 & 11.27 \\
\hline 7.633 & 3.493 & 91.42 & 290.52 & 3481.6 & 102.82 & 28248 & 0.664 & 1.10 & 0.52 & 11.2 \\
\hline
\end{tabular}


Table 3 (continued)

Channel Specimen

Experiment 1

Date: 23 July 1990

Time: 10:26:33

$\begin{array}{cccccccc}\text { TA } & \text { TB } & M & \text { PO } & \text { PO-P1 } & \text { Vf } & f & \text { Wf } \\ K & K & \mathrm{~kg} / \mathrm{h} & \mathrm{KPa} & \mathrm{KPa} & \text { X } & & \text { X } \\ 291.56 & 291.60 & 16.77 & 3586.9 & 16.84 & 0.00 & 0.00547 & 17.14\end{array}$

Hot-side Temperatures:

$\begin{array}{ccc}X & Y & T W \\ \mathrm{~cm} & \mathrm{~cm} & K \\ 1.588 & 0.655 & 291.57 \\ 2.540 & 0.655 & 291.59 \\ 5.080 & 0.655 & 291.58 \\ 7.620 & 0.655 & 291.58 \\ 10.160 & 0.655 & 291.58 \\ 12.700 & 0.655 & 291.60 \\ 13.653 & 0.655 & 291.61\end{array}$

Insulated-side Temperatures and Calculated Data:

\begin{tabular}{|c|c|c|c|c|c|c|c|c|c|c|}
\hline \multirow[b]{2}{*}{$x$} & \multirow[b]{2}{*}{$Y$} & \multirow[b]{2}{*}{$T H$} & \multirow[b]{2}{*}{ Tf } & \multirow[b]{2}{*}{$\mathbf{P}$} & \multirow[b]{2}{*}{ v } & \multirow[b]{2}{*}{ RE } & \multirow[b]{2}{*}{ PR } & \multicolumn{3}{|c|}{ - Uncertainties.- } \\
\hline & & & & & & & & Wtw & Wtf & Wre \\
\hline $\mathrm{cm}$ & $\mathrm{cm}$ & $\mathbf{k}$ & K & $\mathrm{KPa}$ & $\mathrm{m} / \mathrm{s}$ & & & K & K & $x$ \\
\hline 0.000 & -0.965 & 291.65 & 291.41 & 3586.9 & 40.48 & 11393 & 0.664 & 1.10 & 0.50 & 11.27 \\
\hline 1.270 & -0.318 & 291.59 & 291.41 & 3585.4 & 40.50 & 11392 & 0.664 & 1.10 & 0.50 & 11.27 \\
\hline 2.527 & -0.330 & 291.61 & 291.41 & 3584.1 & 40.51 & 11392 & 0.664 & 1.10 & 0.50 & 11.27 \\
\hline 3.810 & -0.318 & 291.61 & 291.42 & 3582.6 & 40.53 & 11392 & 0.664 & 1.10 & 0.50 & 11.27 \\
\hline 5.080 & -0.318 & 291.60 & 291.42 & 3581.2 & 40.55 & 11392 & 0.664 & 1.10 & 0.50 & 11.27 \\
\hline 6.363 & -0.318 & 291.61 & 291.42 & 3579.8 & 40.56 & 11392 & 0.664 & 1.10 & 0.50 & 11.27 \\
\hline 7.620 & -0.330 & 291.63 & 291.42 & 3578.4 & 40.58 & 11392 & 0.664 & 1.10 & 0.50 & 11.27 \\
\hline 8.903 & -0.305 & 291.61 & 291.43 & $35 \pi 7.0$ & 40.60 & 11392 & 0.664 & 1.10 & 0.50 & 11.27 \\
\hline 10.160 & -0.318 & 291.60 & 291.43 & 3575.6 & 40.61 & 11392 & 0.664 & 1.10 & 0.50 & 11.27 \\
\hline 11.430 & -0.318 & 291.60 & 291.43 & 3574.2 & 40.63 & 11392 & 0.664 & 1.10 & 0.50 & 11.27 \\
\hline 12.700 & -0.305 & 291.61 & 291.43 & 3572.8 & 40.64 & 11392 & 0.664 & 1.10 & 0.50 & 11.27 \\
\hline 13.970 & -0.318 & 291.64 & 291.44 & 3571.4 & 40.66 & 11392 & 0.664 & 1.10 & 0.50 & 11.27 \\
\hline 15.240 & -0.953 & 291.63 & 291.44 & 3570.0 & 40.67 & 11392 & 0.664 & 1.10 & 0.50 & 11.27 \\
\hline 2.527 & -2.223 & 291.61 & 291.41 & 3584.1 & 40.51 & 11392 & 0.664 & 1.10 & 0.50 & 11.27 \\
\hline 5.080 & -2.235 & 291.63 & 291.42 & 3581.2 & 40.55 & 11392 & 0.664 & 1.10 & 0.50 & 11.27 \\
\hline 7.607 & -2.235 & 291.64 & 291.42 & 3578.4 & 40.58 & 11392 & 0.664 & 1.10 & 0.50 & 11.27 \\
\hline 10.173 & -2.223 & 291.62 & 291.43 & 3575.6 & 40.61 & 11392 & 0.664 & 1.10 & 0.50 & 11.27 \\
\hline 12.700 & -2.223 & 291.62 & 291.43 & 3572.8 & 40.64 & 11392 & 0.664 & 1.10 & 0.50 & 11.27 \\
\hline 2.540 & 2.197 & 291.63 & 291.41 & 3584.0 & 40.51 & 11392 & 0.664 & 1.10 & 0.50 & 11.27 \\
\hline 5.080 & 2.223 & 291.64 & 291.42 & 3581.2 & 40.55 & 11392 & 0.664 & 1.10 & 0.50 & 11.27 \\
\hline 7.620 & 2.223 & 291.61 & 291.42 & 3578.4 & 40.58 & 11392 & 0.664 & 1.10 & 0.50 & 11.27 \\
\hline 10.147 & 2.223 & 291.61 & 291.43 & 3575.6 & 40.61 & 11392 & 0.664 & 1.10 & 0.50 & 11.27 \\
\hline 12.713 & 2.223 & 291.63 & 291.43 & 3572.8 & 40.64 & 11392 & 0.664 & 1.10 & 0.50 & 11.27 \\
\hline 7.607 & -3.493 & 291.63 & 291.42 & 3578.4 & 40.58 & 11392 & 0.664 & 1.10 & 0.50 & 11.27 \\
\hline 7.633 & 3.493 & 291.64 & 291.42 & 3578.4 & 40.58 & 11392 & 0.664 & 1.10 & 0.50 & 11.27 \\
\hline
\end{tabular}


Channel specimen

Experiment 2

Date: 6 August 1990

Time: 09:02:08

$\begin{array}{cccccccc}T A & T B & M & P O & P 0-P 1 & V f & f & \text { Hf } \\ K & K & k g / h & K P a & K P a & \% & & \% \\ 296.73 & 296.07 & 2.37 & 7219.3 & 0.33 & -0.02 & 0.01132 & 44.97\end{array}$

Hot-side Temperatures:

$\begin{array}{ccc}X & Y & T W \\ \mathrm{~cm} & \mathrm{~cm} & K \\ 1.588 & 0.655 & 296.40 \\ 2.540 & 0.655 & 296.41 \\ 5.080 & 0.655 & 296.37 \\ 7.620 & 0.655 & 296.32 \\ 10.160 & 0.655 & 296.29 \\ 12.700 & 0.655 & 296.27 \\ 13.653 & 0.655 & 296.25\end{array}$

Insulated-Side Temperatures and Calculated Dato:

\begin{tabular}{|c|c|c|c|c|c|c|c|c|c|c|}
\hline \multirow[b]{2}{*}{$x$} & \multirow[b]{2}{*}{ Y } & \multirow[b]{2}{*}{$T w$} & \multirow[b]{2}{*}{ Tf } & \multirow[b]{2}{*}{$P$} & \multirow[b]{2}{*}{ v } & \multirow[b]{2}{*}{ RE } & \multirow[b]{2}{*}{ PR } & \multicolumn{3}{|c|}{ - -Uncertainties-- } \\
\hline & & & & & & & & Wtw & Wtf & Wre \\
\hline $\mathrm{cm}$ & $\mathrm{cm}$ & $k$ & $k$ & $\mathrm{kPa}$ & $\mathrm{m} / \mathrm{s}$ & & & k & $k$ & $x$ \\
\hline 0.000 & -0.965 & 296.50 & 296.73 & 7219.3 & 2.83 & 1523 & 0.661 & 1.10 & 0.50 & 11.27 \\
\hline 1.270 & -0.318 & 296.44 & 296.68 & 7219.3 & 2.83 & 1523 & 0.661 & 1.10 & 0.50 & 11.27 \\
\hline 2.527 & -0.330 & 296.45 & 296.63 & 7219.2 & 2.83 & 1523 & 0.661 & 1.10 & 0.50 & 11.27 \\
\hline 3.810 & -0.318 & 296.40 & 296.57 & 7219.2 & 2.83 & 1523 & 0.661 & 1.10 & 0.50 & 11.27 \\
\hline 5.080 & -0.318 & 296.39 & 296.52 & 7219.2 & 2.83 & 1523 & 0.661 & 1.10 & 0.50 & 11.27 \\
\hline 6.363 & -0.318 & 296.36 & 296.47 & 7219.2 & 2.83 & 1523 & 0.661 & 1.10 & 0.50 & 11.27 \\
\hline 7.620 & -0.330 & 296.36 & 296.42 & 7219.1 & 2.83 & 1524 & 0.661 & 1.10 & 0.50 & 11.27 \\
\hline 8.903 & -0.305 & 296.33 & 296.36 & 7219.1 & 2.83 & 1524 & 0.661 & 1.10 & 0.50 & 11.27 \\
\hline 10.160 & -0.318 & 296.34 & 296.31 & 7219.1 & 2.83 & 1524 & 0.661 & 1.10 & 0.50 & 11.27 \\
\hline 11.430 & -0.318 & 296.31 & 296.26 & 7219.0 & 2.83 & 1524 & 0.661 & 1.10 & 0.50 & 11.27 \\
\hline 12.700 & -0.305 & 296.28 & 296.20 & 7219.0 & 2.83 & 1524 & 0.661 & 1.10 & 0.50 & 11.27 \\
\hline 13.970 & -0.318 & 296.26 & 296.15 & 7219.0 & 2.83 & 1524 & 0.661 & 1.10 & 0.50 & 11.27 \\
\hline 15.240 & -0.953 & 296.22 & 296.10 & 7219.0 & 2.83 & 1525 & 0.661 & 1.10 & 0.50 & 11.27 \\
\hline 2.527 & -2.223 & 296.48 & 296.63 & 7219.2 & 2.83 & 1523 & 0.661 & 1.10 & 0.50 & 11.27 \\
\hline 5.080 & -2.235 & 296.32 & 296.52 & 7219.2 & 2.83 & 1523 & 0.661 & 1.10 & 0.50 & 11.27 \\
\hline 7.607 & -2.235 & 296.37 & 296.42 & 7219.1 & 2.83 & 1524 & 0.661 & 1.10 & 0.50 & 11.27 \\
\hline 10.173 & -2.223 & 296.36 & 296.31 & 7219.1 & 2.83 & 1524 & 0.661 & 1.10 & 0.50 & 11.27 \\
\hline 12.700 & -2.223 & 296.29 & 296.20 & 7219.0 & 2.83 & 1524 & 0.661 & 1.10 & 0.50 & 11.27 \\
\hline 2.540 & 2.197 & 296.42 & 296.63 & 7219.2 & 2.83 & 1523 & 0.661 & 1.10 & 0.50 & 11.27 \\
\hline 5.080 & 2.223 & 296.37 & 296.52 & 7219.2 & 2.83 & 1523 & 0.661 & 1.10 & 0.50 & 11.27 \\
\hline 7.620 & 2.223 & 296.35 & 296.42 & 7219.1 & 2.83 & 1524 & 0.661 & 1.10 & 0.50 & 11.27 \\
\hline 10.147 & 2.223 & 296.33 & 296.31 & 7219.1 & 2.83 & 1524 & 0.661 & 1.10 & 0.50 & 11.27 \\
\hline 12.713 & 2.223 & 296.28 & 296.20 & 7219.0 & 2.83 & 1524 & 0.661 & 1.10 & 0.50 & 11.27 \\
\hline 7.607 & -3.493 & 296.40 & 296.42 & 7219.1 & 2.83 & 1524 & 0.661 & 1.10 & 0.50 & 11.27 \\
\hline 7.633 & 3.493 & 296.36 & 296.41 & 7219.1 & 2.83 & 1524 & 0.661 & 1.10 & 0.50 & 11.27 \\
\hline
\end{tabular}




\section{Table 3 (continued)}

Channel specimen

Experiment 2

Date: 6 August 1990

Time: 09:06:38

$\begin{array}{cccccccc}\text { TA } & \text { TB } & M & P O & P O-P 1 & V f & f & \text { Uf } \\ K & K & k g / h & K P \& & K P 8 & \% & & \% \\ 298.28 & 297.22 & 5.27 & 7210.7 & 1.02 & -0.02 & 0.00697 & 21.73\end{array}$

Hot-side Temperatures:

$\begin{array}{ccc}X & Y & T W \\ \mathrm{~cm} & \mathrm{~cm} & K \\ 1.588 & 0.655 & 297.83 \\ 2.540 & 0.655 & 297.82 \\ 5.080 & 0.655 & 297.78 \\ 7.620 & 0.655 & 297.76 \\ 10.160 & 0.655 & 297.73 \\ 12.700 & 0.655 & 297.68 \\ 13.653 & 0.655 & 297.67\end{array}$

Insulated-Side Temperatures and Calculated Data:

\begin{tabular}{|c|c|c|c|c|c|c|c|c|c|c|}
\hline & & & & & & & & & & \\
\hline$x$ & $Y$ & $T w$ & Tf & $P$ & v & RE & PR & Wtw & Wtf & Wre \\
\hline $\mathrm{cm}$ & $\mathrm{cm}$ & K & $K$ & $\mathrm{kPa}$ & $\mathrm{m} / \mathrm{s}$ & & & $K$ & K & $x$ \\
\hline 0.000 & -0.965 & 297.96 & 298.28 & 7210.7 & 6.38 & 3398 & 0.661 & 1.10 & 0.50 & 11.27 \\
\hline 1.270 & -0.318 & 297.98 & 298.20 & 7210.6 & 6.38 & 3399 & 0.661 & 1.10 & 0.50 & 11.27 \\
\hline 2.527 & -0.330 & 297.91 & 298.11 & 7210.5 & 6.38 & 3400 & 0.661 & 1.10 & 0.50 & 11.27 \\
\hline 3.810 & -0.318 & 297.85 & 298.03 & 7210.4 & 6.38 & 3400 & 0.661 & 1.10 & 0.50 & 11.27 \\
\hline 5.080 & -0.318 & 297.85 & 297.94 & 7210.3 & 6.38 & 3401 & 0.661 & 1.10 & 0.50 & 11.27 \\
\hline 6.363 & -0.318 & 297.86 & 297.86 & 7210.2 & 6.37 & 3401 & 0.661 & 1.10 & 0.50 & 11.27 \\
\hline 7.620 & -0.330 & 297.85 & 297.77 & 7210.2 & 6.37 & 3402 & 0.661 & 1.10 & 0.50 & 11.27 \\
\hline 8.903 & -0.305 & 297.81 & 297.69 & 7210.1 & 6.37 & 3403 & 0.661 & 1.10 & 0.50 & 11.27 \\
\hline 10.160 & -0.318 & 297.83 & 297.60 & 7210.0 & 6.37 & 3403 & 0.661 & 1.10 & 0.50 & 11.27 \\
\hline 11.430 & -0.318 & 297.81 & 297.52 & 7209.9 & 6.37 & 3404 & 0.661 & 1.10 & 0.50 & 11.27 \\
\hline 12.700 & -0.305 & 297.79 & 297.43 & 7209.8 & 6.36 & 3405 & 0.661 & 1.10 & 0.50 & 11.27 \\
\hline 13.970 & -0.318 & 297.74 & 297.35 & 7209.7 & 6.36 & 3405 & 0.661 & 1.10 & 0.50 & 11.27 \\
\hline 15.240 & -0.953 & 297.69 & 297.26 & 7209.6 & 6.36 & 3406 & 0.661 & 1.10 & 0.50 & 11.27 \\
\hline 2.527 & -2.223 & 298.04 & 298.11 & 7210.5 & 6.38 & 3400 & 0.661 & 1.10 & 0.50 & 11.27 \\
\hline 5.080 & -2.235 & 297.64 & 297.94 & 7210.3 & 6.38 & 3401 & 0.661 & 1.10 & 0.50 & 11.27 \\
\hline 7.607 & -2.235 & 297.86 & 297.77 & 7210.2 & 6.37 & 3402 & 0.661 & 1.10 & 0.50 & 11.27 \\
\hline 10.173 & -2.223 & 297.89 & 297.60 & 7210.0 & 6.37 & 3403 & 0.661 & 1.10 & 0.50 & 11.27 \\
\hline 12.700 & -2.223 & 297.86 & 297.43 & 7209.8 & 6.36 & 3405 & 0.661 & 1.10 & 0.50 & 11.27 \\
\hline 2.540 & 2.197 & 297.86 & 298.11 & 7210.5 & 6.38 & 3400 & 0.661 & 1.10 & 0.50 & 11.27 \\
\hline 5.080 & 2.223 & 297.86 & 297.94 & 7210.3 & 6.38 & 3401 & 0.661 & 1.10 & 0.50 & 11.27 \\
\hline 7.620 & 2.223 & 297.82 & 297.77 & 7210.2 & 6.37 & 3402 & 0.661 & 1.10 & 0.50 & 11.27 \\
\hline 10.147 & 2.223 & 297.81 & 297.60 & 7210.0 & 6.37 & 3403 & 0.661 & 1.10 & 0.50 & 11.27 \\
\hline 12.713 & 2.223 & 297.77 & 297.43 & 7209.8 & 6.36 & 3405 & 0.661 & 1.10 & 0.50 & 11.27 \\
\hline 7.607 & -3.493 & 297.98 & 297.77 & 7210.2 & 6.37 & 3402 & 0.661 & 1.10 & 0.50 & 11.27 \\
\hline 7.633 & 3.493 & 297.81 & 297.77 & 7210.2 & 6.37 & 3402 & 0.661 & 1.10 & 0.50 & 11.27 \\
\hline
\end{tabular}




\section{Table 3 (continued)}

Channel Specimen

Experiment 2

Date: 6 August 1990

Time: $09: 11: 11$

\begin{tabular}{|c|c|c|c|c|c|c|c|}
\hline TA & TB & H & PO & P0-P1 & $V f$ & $f$ & Wf \\
\hline K & K & $\mathrm{kg} / \mathrm{h}$ & $K P_{B}$ & $\mathrm{kPa}$ & $x$ & & \\
\hline .6 & 299.05 & 10.23 & 7212.6 & 3.51 & -0.01 & 0.00613 & 7. \\
\hline
\end{tabular}

Hot-side Temperatures:

$\begin{array}{ccc}X & Y & T H \\ \mathrm{~cm} & \mathrm{~cm} & K \\ 1.588 & 0.655 & 299.32 \\ 2.540 & 0.655 & 299.34 \\ 5.080 & 0.655 & 299.31 \\ 7.620 & 0.655 & 299.25 \\ 10.160 & 0.655 & 299.26 \\ 12.700 & 0.655 & 299.24 \\ 13.653 & 0.655 & 299.23\end{array}$

Insulated-Side Temperatures and Calculated Data:

\begin{tabular}{|c|c|c|c|c|c|c|c|c|c|c|}
\hline & & & & & & & & & & \\
\hline$x$ & 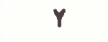 & TH & If & $\mathbf{P}$ & 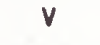 & RE & PR & WtH & Wef & Hre \\
\hline $\mathrm{cm}$ & $\mathrm{cm}$ & $\%$ & K & $\mathrm{kPa}$ & $\mathrm{m} / \mathrm{s}$ & & & $\%$ & K & $x$ \\
\hline .000 & .965 & 9.41 & 99.60 & 12.6 & 2.63 & 6678 & 0 & & 0.50 & 1.27 \\
\hline .27 & .3 & & - & & & & & & & \\
\hline 527 & 0 & 6 & 1 & & & 679 & . & & 50 & 1.27 \\
\hline 810 & .31 & 9.31 & 299.46 & & 2.62 & 680 & 0.661 & 10 & .50 & 1.27 \\
\hline .080 & -0.318 & 9.31 & 299.42 & & & 6680 & 0.6 & 10 & 50 & 1.27 \\
\hline 363 & $-c$ & 3 & 299 & & & 81 & & & & 1.27 \\
\hline 620 & 0.00 & 9.31 & 299.33 & & & 82 & & & 0 & 1.27 \\
\hline .903 & -0.305 & 99.29 & 299.29 & & 2 & 682 & 0.6 & 10 & .50 & 1.27 \\
\hline .160 & -0.318 & 99.30 & 299.24 & & & 6683 & 0. & & 30 & 1.27 \\
\hline .430 & -0.3 & 0 & 209 & & & 584 & & & & 1.27 \\
\hline .700 & -0.305 & 299.29 & 299. & & & 4 & & & & 1.27 \\
\hline .970 & -0.318 & 299.27 & 299. & 72 & 12. & 6685 & 0.6 & & 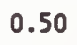 & 1.27 \\
\hline .240 & -0.953 & 299.26 & 299. & 72 & 2. & 6685 & 0. & & 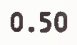 & 1.27 \\
\hline 2.527 & -2.223 & 209,40 & 99.51 & & & 79 & & & & 1.27 \\
\hline .080 & 2.2 & 299.04 & & & & & & & & 1.27 \\
\hline 7.607 & -2.235 & 299. & (7.0.0 & & & & & & & .27 \\
\hline .173 & -2.223 & 299.33 & 99.24 & & & & & & & 1.27 \\
\hline .700 & -2.223 & 299. & 9.16 & & & 84 & & & 0 & 1.27 \\
\hline 2.540 & 2.197 & 299.23 & 299.51 & & & 79 & & & 50 & 1.27 \\
\hline 5.080 & 2.223 & 299. & n & & & 6680 & & & 0 & 1.27 \\
\hline .620 & & & & & & 6682 & & & 50 & 11.27 \\
\hline 1.147 & 2.223 & 299.22 & & & & 6683 & & & 0.50 & 1.27 \\
\hline 2.713 & 2.223 & 299.17 & 299.16 & & & 6684 & 0.661 & 10 & 0.50 & 1.27 \\
\hline 7.607 & -3.493 & 299.36 & & & & 6682 & 0. & 0 & 0.50 & 1.27 \\
\hline & 5.495 & 99.15 & 99.33 & 7210.9 & 12.62 & 6682 & 0.661 & 1.16 & 0.50 & 11.8 \\
\hline
\end{tabular}




\section{Table 3 (continued)}

Channel Specimen

Experiment 2

Date: 6 August 1990

Time: 09:16:04

$\begin{array}{cccccccc}T A & T B & M & P O & P O-P 1 & V f & f & \text { Uf } \\ K & K & \mathrm{~kg} / \mathrm{h} & \mathrm{KPa} & \mathrm{kPa} & \mathrm{X} & & \% \\ 298.75 & 298.83 & 14.54 & 7224.6 & 6.57 & 0.00 & 0.00557 & 17.24\end{array}$

Hot-side Temperatures:

$\begin{array}{ccc}X & Y & T H \\ \mathrm{~cm} & \mathrm{~cm} & K \\ 1.588 & 0.655 & 298.65 \\ 2.540 & 0.655 & 298.66 \\ 5.080 & 0.655 & 298.64 \\ 7.620 & 0.655 & 298.62 \\ 10.160 & 0.655 & 298.62 \\ 12.700 & 0.655 & 298.61 \\ 13.653 & 0.655 & 298.61\end{array}$

Insulated-Side Temperatures and Calculated Data:

\begin{tabular}{|c|c|c|c|c|c|c|c|c|c|c|}
\hline & & & & & & & & & & \\
\hline$x$ & $Y$ & $T H$ & Tf & P & $v$ & RE & PR & HeH & Wtf & Wre \\
\hline $\mathrm{cm}$ & $\mathrm{Cr}$ & $K$ & $k$ & $\mathrm{KPa}$ & $\mathrm{m} / \mathrm{s}$ & & & K & K & $x$ \\
\hline 0.000 & -0.965 & 298.63 & 298.72 & 7224.6 & 18.06 & 9613 & 0.661 & 1.10 & 0.50 & 11.27 \\
\hline 1.270 & -0.318 & 298.61 & 298.73 & 7224.1 & 18.06 & 9612 & 0.661 & 1.10 & 0.50 & 11.27 \\
\hline 2.527 & -0.330 & 298.59 & 298.73 & 7223.5 & 18.06 & 9612 & 0.661 & 1.10 & 0.50 & 11.27 \\
\hline 3.810 & -0.318 & 298.58 & 298.74 & 7223.0 & 18.07 & 9612 & 0.661 & 1.10 & 0.50 & 11.27 \\
\hline 5.080 & -0.318 & 298.57 & 298.75 & 7222.4 & 18.07 & 9612 & 0.661 & 1.10 & 0.50 & 11.27 \\
\hline 6.363 & -0.318 & 298.58 & 298.75 & 7221.9 & 18.07 & 9612 & 0.661 & 1.10 & 0.50 & 11.27 \\
\hline 7.620 & -0.330 & 298.58 & 298.76 & 7221.4 & 18.07 & 9612 & 0.661 & 1.10 & 0.50 & 11.27 \\
\hline 8.903 & -0.305 & 298.55 & 298.76 & 7220.8 & 18.07 & 9612 & 0.661 & 1.10 & 0.50 & 11.27 \\
\hline 10.160 & -0.318 & 298.53 & 298.77 & 7220.3 & 18.07 & 9612 & 0.661 & 1.10 & 0.50 & 11.27 \\
\hline 11.430 & -0.318 & 298.53 & 298.77 & 7219.7 & 18.08 & 9612 & 0.661 & 1.10 & 0.50 & 11.27 \\
\hline 12.700 & -0.305 & 298.54 & 298.78 & 7219.2 & 18.08 & 9611 & 0.661 & 1.10 & 0.50 & 11.27 \\
\hline 13.970 & -0.318 & 298.53 & 298.79 & 7218.6 & 18.08 & 9611 & 0.661 & 1.10 & 0.50 & 11.27 \\
\hline 15.240 & -0.953 & 298.55 & 298.79 & 7218.1 & 18.08 & 9611 & 0.661 & 1.10 & 0.50 & 11.27 \\
\hline 2.527 & -2.223 & 298.52 & 298.73 & 7223.5 & 18.06 & 9612 & 0.661 & 1.10 & 0.50 & 11.27 \\
\hline 5.080 & -2.235 & 298.41 & 298.75 & 7222.4 & 18.07 & 9612 & 0.661 & 1.10 & 0.50 & 11.27 \\
\hline 7.607 & -2.235 & 298.51 & 298.76 & 7221.4 & 18.07 & 9612 & 0.661 & 1.10 & 0.50 & 11.27 \\
\hline 10.173 & -2.223 & 298.48 & 298.77 & 7220.3 & 18.07 & 9612 & 0.661 & 1.10 & 0.50 & 11.27 \\
\hline 12.700 & -2.223 & 298.48 & 298.78 & 7219.2 & 18.08 & 9611 & 0.661 & 1.10 & 0.50 & 11.27 \\
\hline 2.540 & 2.197 & 298.45 & 298.73 & 7223.5 & 18.06 & 9612 & 0.661 & 1.10 & 0.50 & 11.27 \\
\hline 5.080 & 2.223 & 298.45 & 298.75 & 7222.4 & 18.07 & 9612 & 0.661 & 1.10 & 0.50 & 11.27 \\
\hline 7.620 & 2.223 & 298.46 & 298.76 & 7221.4 & 18.07 & 9612 & 0.661 & 1.10 & 0.50 & 11.27 \\
\hline 10.147 & 2.223 & 298.47 & 298.77 & 7220.3 & 18.07 & 9612 & 0.661 & 1.10 & 0.50 & 11.27 \\
\hline 12.713 & 2.223 & 298.45 & 298.78 & 7219.2 & 18.08 & 9611 & 0.661 & 1.10 & 0.50 & 11.27 \\
\hline 7.607 & -3.493 & 298.45 & 298.76 & 7221.4 & 18.07 & 9612 & 0.661 & 1.10 & 0.50 & 11.27 \\
\hline 7.633 & 3.493 & 298.41 & 298.76 & 7221.3 & 18.07 & 9612 & 0.661 & 1.10 & 0.50 & 11.27 \\
\hline
\end{tabular}




\section{Table 3 (continued)}

Channel specimen

Experiment 2

Date: 6 August 1990

Time: 09:20:49

$\begin{array}{cccccccc}\text { TA } & T B & M & P O & P O-P 1 & V f & f & \text { Wf } \\ K & K & \mathrm{~kg} / \mathrm{h} & \mathrm{KPa} & \mathrm{KPa} & \mathrm{X} & & \% \\ 297.61 & 297.76 & 20.12 & 7244.1 & 11.74 & -0.02 & 0.00519 & 17.16\end{array}$

Hot-side Temperotures:

$\begin{array}{ccc}X & Y & T W \\ \mathrm{~cm} & \mathrm{~cm} & K \\ 1.588 & 0.655 & 297.63 \\ 2.540 & 0.655 & 297.61 \\ 5.080 & 0.655 & 297.60 \\ 7.620 & 0.655 & 297.59 \\ 10.160 & 0.655 & 297.58 \\ 12.700 & 0.655 & 297.60 \\ 13.653 & 0.655 & 297.58\end{array}$

Insulated-Side Temperatures and Calculated Data:

\begin{tabular}{|c|c|c|c|c|c|c|c|c|c|c|}
\hline & & & & & & & & \multicolumn{3}{|c|}{ - Uncertainties - } \\
\hline$x$ & r & TW & Tf & $\mathbf{P}$ & v & RE & PR & Wtw & Wef & Wre \\
\hline $\mathrm{cm}$ & $\mathrm{cm}$ & $\mathbf{R}$ & K & $\mathrm{kPa}$ & $\mathrm{m} / \mathrm{s}$ & & & $\mathbf{R}$ & $K$ & $x$ \\
\hline 0.000 & -0.965 & 97.58 & 297.55 & 7244.1 & 25.12 & 13487 & 0.661 & 1.10 & 0.50 & 11.27 \\
\hline 1.270 & -0.318 & 297.58 & 297.56 & 7243.2 & 25.12 & 13487 & 0.661 & 1.10 & 0.50 & 19.27 \\
\hline 2.527 & -0.330 & 297.54 & 297.57 & 7242.2 & 25.12 & 13486 & 0.661 & 1.10 & 0.50 & 11.27 \\
\hline 3.810 & -0.318 & 297.55 & 297.58 & 7241.2 & 25.13 & 13486 & 0.661 & 1.10 & 0.50 & 11.27 \\
\hline 5.080 & -0.318 & 297.54 & 297.60 & 7240.2 & 25.13 & 13486 & 0.661 & 1.10 & 0.50 & 11.27 \\
\hline 6.363 & -0.318 & 297.55 & 297.61 & 7239.2 & 25.14 & 13485 & 0.661 & 1.10 & 0.50 & 1.27 \\
\hline 7.620 & -0.330 & 297.55 & 297.62 & 7238.3 & 25.14 & 13485 & 0.661 & .10 & .50 & 1.27 \\
\hline 8.903 & -0.305 & 297.55 & 297.63 & 7237.3 & 25.15 & 13485 & 0.661 & 1.10 & .50 & 1.27 \\
\hline 10.160 & -0.318 & 297.53 & 297.64 & 7236.3 & 5 & 13484 & 0.661 & 0 & .50 & .27 \\
\hline 11.430 & $=0$ & 297.53 & 297. & 72 & 5 & 13484 & 0.6 & & .50 & .27 \\
\hline 12.700 & -0.305 & 297.52 & 67 & 7 & & 13484 & 1 & 1.10 & .50 & .27 \\
\hline 13.970 & -0. & 297.54 & 2 & 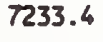 & 6 & 13483 & .6 & 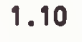 & .50 & .27 \\
\hline 15.240 & -0 . & 297 & 297 & 72 & 7 & 13483 & 0.661 & 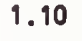 & .50 & 9.27 \\
\hline 2.527 & -2.223 & 297.53 & 297.57 & 72 & 2 & 13486 & 0.661 & 0 & .50 & 11.27 \\
\hline 5.080 & -2.235 & 297.49 & 297.60 & 724 & 25.13 & 13486 & 0.661 & 0 & 0.50 & 11.27 \\
\hline 7.607 & -2.235 & 297.51 & 297.62 & 7238.3 & 25.14 & 13485 & 0.661 & 1.10 & 0.50 & 11.27 \\
\hline 10.173 & -2.223 & 297.52 & 297.64 & 7236.3 & 25.15 & 13484 & 0.661 & 1.10 & 0.50 & 11.27 \\
\hline 12.700 & -2.223 & 297.49 & 297.67 & 7234.4 & 25.16 & 13484 & 0.661 & 1.10 & 0.50 & 11.27 \\
\hline 2.540 & 2.197 & 297.55 & 297.57 & 7242.2 & 25.12 & 13486 & 0.661 & 1.10 & 0.50 & 11.27 \\
\hline 5.080 & 2.223 & 297.54 & 297.60 & 7240.2 & 25.13 & 13486 & 0.661 & 1.10 & 0.50 & 11.27 \\
\hline 7.620 & 2.223 & 297.52 & 297.62 & 7238.3 & 25.14 & 13485 & 0.661 & 1.10 & 0.50 & 11.27 \\
\hline 10.147 & 2.223 & 297.53 & 297.64 & 7236.3 & 25.15 & 13484 & 0.661 & 1.10 & 0.50 & 11.27 \\
\hline 12.713 & 2.223 & 297.55 & 297.67 & 7234.3 & 25.16 & 13484 & 0.661 & 1.10 & 0.50 & 11.27 \\
\hline 7.607 & $\cdot 3.493$ & 297.49 & 297.62 & 7238.3 & 25.14 & 13485 & 0.661 & 1.10 & 0.50 & 11.27 \\
\hline 7.633 & 3.493 & 297.52 & 297.62 & 7238.3 & 25.14 & 13485 & 0.661 & 1.10 & 0.50 & 11.27 \\
\hline
\end{tabular}




\section{Table 3 (continued)}

Channel specimen

Experiment 2

Date: 6 August 1990

Time: $09: 24: 57$

$\begin{array}{cccccccc}\text { TA } & T B & M & P O & P 0 . P 1 & V f & f & \text { Hf } \\ K & K & \mathrm{~kg} / \mathrm{h} & \mathrm{kPa} & \mathrm{kPa} & \boldsymbol{K} & & \boldsymbol{K}\end{array}$

$\begin{array}{llllllll}297.14 & 297.26 & 25.60 & 7253.4 & 18.03 & .0 .01 & 0.00476 & 17.14\end{array}$

Hot-side Temperatures:

$\begin{array}{ccc}X & Y & T H \\ \mathrm{~cm} & \mathrm{~cm} & K \\ 1.588 & 0.655 & 297.10 \\ 2.540 & 0.655 & 297.07 \\ 5.080 & 0.655 & 297.10 \\ 7.620 & 0.655 & 297.09 \\ 10.160 & 0.655 & 297.07 \\ 12.700 & 0.655 & 297.09 \\ 13.653 & 0.655 & 297.08\end{array}$

Insulated-side Temperatures and Calculated Data:

\begin{tabular}{|c|c|c|c|c|c|c|c|c|c|c|}
\hline \multirow[b]{2}{*}{$x$} & & \multirow[b]{2}{*}{ Tw } & \multirow[b]{2}{*}{ Tf } & & \multirow[b]{2}{*}{ v } & \multirow[b]{2}{*}{ RE } & \multirow[b]{2}{*}{ PR } & \multicolumn{3}{|c|}{--Uncertainties.- } \\
\hline & & & & & & & & WEH & Wtf & Wre \\
\hline $\mathrm{cm}$ & $\mathrm{cm}$ & $x$ & 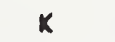 & $\mathrm{kPa}$ & $\mathrm{m} / \mathrm{s}$ & & & $k$ & $\boldsymbol{k}$ & \% \\
\hline 0.000 & 0.965 & 297.07 & 297.05 & 7253.4 & 32.17 & 17341 & 0.661 & 1.10 & 0.50 & 11.27 \\
\hline 1.270 & 0.318 & 97.04 & 97.05 & 7251.8 & 2.17 & 17340 & 0.661 & .10 & 0.50 & 11.27 \\
\hline 2.527 & -0.330 & 297.04 & 97.06 & 7250.4 & 2.18 & 17340 & 0.661 & .10 & 0.50 & 11.27 \\
\hline 3.810 & $\cdot 0.318$ & 297.04 & 97.07 & 7248.8 & 2.19 & 17340 & 0.661 & .10 & 0.50 & 11.27 \\
\hline 5.080 & -0.318 & 297.05 & 297.08 & 7247.3 & 32.19 & 17340 & 0.661 & .10 & 0.50 & 11.27 \\
\hline 6.363 & -0.318 & 297.02 & 297.09 & 7245.8 & 32.20 & 17339 & 0.661 & .10 & .50 & 11.27 \\
\hline 7.620 & -0.330 & 297.01 & 297.10 & 7244.3 & 32.21 & 17339 & 0.66 & 10 & .50 & 11.27 \\
\hline 8.903 & -0.305 & 297.05 & 297.11 & 7242.8 & 32.22 & 17339 & 0.66 & 10 & .50 & 11.27 \\
\hline .160 & -0.318 & 297.03 & 297.11 & 7241.3 & 32.22 & 17338 & 0.66 & 10 & 0.50 & 11.27 \\
\hline 1.430 & -0.318 & 297.03 & 297.12 & 723 & 32.23 & 17338 & 0.6 & .10 & 50 & 11.27 \\
\hline .700 & -0.305 & 297.02 & 297.13 & 7238.3 & 32.24 & 17338 & 0.66 & .10 & 0.50 & 11.27 \\
\hline .970 & $\cdot 0.318$ & 297.03 & 297.14 & 7236.8 & 32.25 & 17338 & 0.661 & 10 & 0.50 & 11.27 \\
\hline 15.240 & -0.953 & 297.04 & 297.15 & 7235.3 & 32.25 & 17337 & 0.661 & .10 & 0.50 & 11.27 \\
\hline 2.527 & $\cdot 2.223$ & 297.01 & 297.06 & 7250.4 & 32.18 & 17340 & 0.6 & 10 & .50 & 11.27 \\
\hline 5.080 & -2.235 & 297.01 & 297.08 & 7247.3 & 32.19 & 17340 & 0.66 & .10 & 0.50 & 11.27 \\
\hline 7.607 & -2.235 & 297.00 & 297.10 & 7244.4 & 32.21 & 17339 & 0.66 & .10 & 0.50 & 11.27 \\
\hline 0.173 & -2.223 & 296.99 & 297.12 & 72 & 32.22 & 17338 & 0.661 & .10 & 0.50 & 11.27 \\
\hline 12.700 & -2.223 & 296.97 & 297.13 & 7238.3 & 32.24 & 17338 & 0.661 & 1.10 & 0.50 & 11.27 \\
\hline 2.540 & 2.197 & 297.01 & 297.06 & 7250.3 & 32.18 & 17340 & 0.66 & 1.10 & 0.50 & 11.27 \\
\hline 5.080 & 2.223 & 296.99 & 97.08 & 7247.3 & 32.19 & 17340 & 0.661 & .10 & 0.50 & 11.27 \\
\hline 7.620 & 2.223 & 297.00 & 297.10 & 7244.3 & 32.21 & 17339 & 0.661 & .10 & 0.50 & 11.27 \\
\hline 10.147 & 2.223 & 296.98 & 297.11 & 7241.3 & 32.22 & 17338 & 0.661 & 1.10 & 0.50 & 11.27 \\
\hline 12.713 & 2.223 & 297.01 & 27.13 & 7238.3 & 32.24 & 17338 & 0.661 & 1.10 & 0.50 & 11.27 \\
\hline 7.607 & -3.493 & 296.96 & 7.10 & 7244.4 & 32.21 & 17339 & 0.661 & 1.10 & 0.50 & 11.27 \\
\hline 7.633 & 3.493 & 296.97 & 7.10 & 7244.3 & 32.21 & 17339 & 0.661 & 1.10 & 0.50 & 11.27 \\
\hline
\end{tabular}




\section{Table 3 (continued)}

Channel Specimen

Experiment 2

Date: 6 August 1990

rime: 09:29:52

$\begin{array}{cccccccc}T A & T B & M & P O & P O-P 1 & V f & f & \text { Wf } \\ K & K & k g / h & K P a & K P a & \boldsymbol{X} & & \text { \% } \\ 296.23 & 296.38 & 29.87 & 7262.5 & 24.85 & -0.01 & 0.00479 & 17.13\end{array}$

Hot-side Temperatures:

$\begin{array}{ccc}X & Y & T H \\ \mathrm{~cm} & \mathrm{~cm} & K \\ 1.588 & 0.655 & 296.18 \\ 2.540 & 0.655 & 296.18 \\ 5.080 & 0.655 & 296.17 \\ 7.620 & 0.655 & 296.18 \\ 10.160 & 0.655 & 296.18 \\ 12.700 & 0.655 & 296.18 \\ 13.653 & 0.655 & 296.19\end{array}$

Insulated-Side Temperatures and Calculated Data:

\begin{tabular}{|c|c|c|c|c|c|c|c|c|c|c|}
\hline & & & & & & & & & & \\
\hline$x$ & $Y$ & $T_{H}$ & If & $P$ & v & RE & PR & HEW & Wef & Hre \\
\hline $\mathrm{cm}$ & $\mathrm{Cm}$ & K & K & $\mathrm{KPa}$ & $\mathrm{m} / \mathrm{s}$ & & & K & K & $x$ \\
\hline 0.000 & -0.965 & 296.14 & 296.10 & 7262.5 & 37.57 & 20384 & 0.661 & 1.10 & 0.50 & 11.27 \\
\hline 1.270 & -0.318 & 296.11 & 296.11 & 7260.4 & .37 .58 & 20383 & 0.661 & 1.10 & 0.50 & 11.27 \\
\hline 2.527 & -0.330 & 296.12 & 296.12 & 7258.4 & 37.60 & 20383 & 0.661 & 1.10 & 0.50 & 11.27 \\
\hline 3.810 & -0.318 & 296.14 & 296.13 & 7256.3 & 37.61 & 20383 & 0.661 & 1.10 & 0.50 & 11.27 \\
\hline 5.080 & -0.318 & 296.13 & 296.14 & 7254.2 & 37.62 & 20382 & 0.661 & 1.10 & 0.50 & 11.27 \\
\hline 6.363 & -0.318 & 296.10 & 296.15 & 7252.1 & 37.63 & 20382 & 0.661 & 1.10 & 0.50 & 11.27 \\
\hline 7.620 & -0.330 & 296.10 & 296.16 & 7250.1 & 37.64 & 20381 & 0.661 & 1.10 & 0.50 & 11.27 \\
\hline 8.903 & -0.305 & 296.10 & 296.17 & 7248.0 & 37.65 & 20381 & 0.661 & 1.10 & 0.50 & 11.27 \\
\hline 10.160 & -0.318 & 296.09 & 296.18 & 7245.9 & 37.67 & 20381 & 0.661 & 1.10 & 0.50 & 11.27 \\
\hline 11.430 & -0.318 & 296.11 & 296.20 & 7243.8 & 37.68 & 20380 & 0.661 & 1.10 & 0.50 & 11.27 \\
\hline 12.700 & -0.305 & 296.10 & 296.21 & 7241.8 & 37.69 & 20380 & 0.661 & 1.10 & 0.50 & 11.27 \\
\hline 13.970 & -0.318 & 296.10 & 296.22 & 7239.7 & 37.70 & 20379 & 0.661 & 1.10 & 0.50 & 11.27 \\
\hline 15.240 & -0.953 & 296.09 & 296.23 & 7237.6 & 37.71 & 20379 & 0.661 & 1.10 & 0.50 & 11.27 \\
\hline 2.527 & -2.223 & 296.07 & 296.12 & 7258.4 & 37.60 & 20383 & 0.661 & 1.10 & 0.50 & 11.27 \\
\hline 5.080 & -2.235 & 296.13 & 296.14 & 7254.2 & 37.62 & 20382 & 0.661 & 1.10 & 0.50 & 11.27 \\
\hline 7.607 & -2.235 & 296.10 & 296.16 & 7250.1 & 37.64 & 20381 & 0.661 & 1.10 & 0.50 & 11.27 \\
\hline 10.173 & -2.223 & 296.06 & 296.18 & 7245.9 & 37.67 & 20381 & 0.661 & 1.10 & 0.50 & 11.27 \\
\hline 12.700 & -2.223 & 296.06 & 296.21 & 7241.8 & 37.69 & 20380 & 0.661 & 1.10 & 0.50 & 11.27 \\
\hline 2.540 & 2.197 & 296.09 & 296.12 & 7258.3 & 37.60 & 20383 & 0.661 & 1.10 & 0.50 & 11.27 \\
\hline 5.080 & 2.223 & 296.08 & 296.14 & 7254.2 & 37.62 & 20382 & 0.661 & 1.10 & 0.50 & 11.27 \\
\hline 7.620 & 2.223 & 296.07 & 296.16 & 7250.1 & 37.64 & 20381 & 0.661 & 1.10 & 0.50 & 11.27 \\
\hline 10.147 & 2.223 & 296.11 & 296.18 & 7245.9 & 37.67 & 20381 & 0.661 & 1.10 & 0.50 & 11.27 \\
\hline 12.713 & 2.223 & 296.07 & 296.21 & 7241.7 & 37.69 & 20380 & 0.661 & 1.10 & 0.50 & 11.27 \\
\hline 7.607 & -3.493 & 296.02 & 296.16 & 7250.1 & 37.64 & 20381 & 0.661 & 1.10 & 0.50 & 11.27 \\
\hline 7.633 & 3.493 & 296.06 & 296.16 & 7250.0 & 37.64 & 20381 & 0.661 & 1.10 & 0.50 & 11.27 \\
\hline
\end{tabular}




\section{Table 3 (continued)}

Channel specimen

Experiment 2

Date: 6 August 1990

Time: $09: 34: 35$

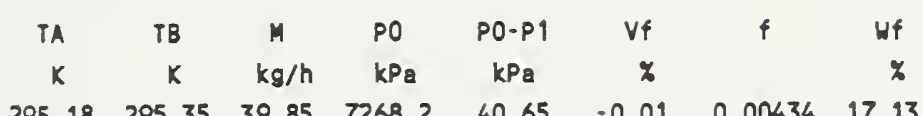

$\begin{array}{llllllll}295.18 & 295.35 & 39.85 & 7268.2 & 40.65 & -0.01 & 0.00434 & 17.13\end{array}$

Hot-side Temperatures:

$\begin{array}{ccc}X & Y & T H \\ \mathrm{~cm} & \mathrm{~cm} & K \\ 1.588 & 0.655 & 295.13 \\ 2.540 & 0.655 & 295.14 \\ 5.080 & 0.655 & 295.13 \\ 7.620 & 0.655 & 295.14 \\ 10.160 & 0.655 & 295.13 \\ 12.700 & 0.655 & 295.16 \\ 13.653 & 0.655 & 295.15\end{array}$

Insulated-Side Temperatures and Calculated Data:

\begin{tabular}{|c|c|c|c|c|c|c|c|c|c|c|}
\hline & & & & & & & & & & $e s=6$ \\
\hline$x$ & $Y$ & TH & Tf & $P$ & $v$ & $R E$ & $P R$ & Wtw & utf & Wre \\
\hline $\mathrm{cm}$ & $\mathrm{cm}$ & $K$ & $k$ & $\mathrm{KPa}$ & $\mathrm{m} / \mathrm{s}$ & & & $K$ & $K$ & $x$ \\
\hline 0.000 & -0.965 & 95.12 & 294.94 & 7268.2 & 50.27 & 27463 & 0.661 & 1.10 & 0.50 & 11.27 \\
\hline 1.270 & -0.318 & 295.07 & 294.95 & 7264.8 & 50.29 & 27462 & 0.661 & 1.10 & 0.50 & 11.27 \\
\hline 2.527 & -0.330 & 295.08 & 294.97 & 7261.5 & 50.31 & 27461 & 0.661 & 1.10 & 0.50 & 11.27 \\
\hline 3.810 & -0.318 & 295.11 & 294.98 & 7258.1 & 50.34 & 27461 & 0.661 & 1.10 & 0.50 & 19.27 \\
\hline 5.080 & -0.318 & 295.09 & 294.99 & 7254.7 & 50.37 & 27460 & 0.661 & 1.10 & 0.50 & 19.27 \\
\hline 6.363 & $\cdot 0.318$ & 295.08 & 295.00 & 7251.3 & 50.39 & 27460 & 0.661 & 1.10 & 0.50 & 19.27 \\
\hline 7.620 & -0.330 & 295.07 & 295.02 & 7247.9 & 50.41 & 27459 & 0.661 & 1.10 & 0.50 & 11.27 \\
\hline 8.903 & -0.305 & 295.09 & 295.03 & 7244.5 & 50.44 & 27459 & 0.661 & 1.10 & 0.50 & 19.27 \\
\hline 10.160 & -0.318 & 295.07 & 295.04 & 7241.1 & 50.46 & 27458 & 0.661 & 1.10 & 0.50 & 11.27 \\
\hline 11.430 & -0.318 & 295.06 & 295.05 & 7237.7 & 50.49 & 27458 & 0.661 & 1.10 & 0.50 & 11.27 \\
\hline 12.700 & -0.305 & 295.07 & 295.06 & 7234.4 & 50.51 & 27457 & 0.661 & 1.10 & 0.50 & 19.27 \\
\hline 13.970 & -0.318 & 295.08 & 295.08 & 7231.0 & 50.54 & 27456 & 0.661 & 1.10 & 0.50 & 11.27 \\
\hline 15.240 & -0.953 & 295.08 & 295.09 & 7227.6 & 50.56 & 27456 & 0.661 & 1.10 & 0.50 & 11.27 \\
\hline 2.527 & -2.223 & 295.04 & 294.97 & 7261.5 & 50.31 & 27461 & 0.661 & 1.10 & 0.50 & 19.27 \\
\hline 5.080 & -2.235 & 295.20 & 294.99 & 7254.7 & 50.37 & 27460 & 0.661 & 1.10 & 0.50 & 99.27 \\
\hline 7.607 & -2.235 & 295.10 & 295.02 & 7247.9 & 50.41 & 27459 & 0.661 & 1.10 & 0.50 & 11.27 \\
\hline 10.173 & -2.223 & 295.05 & 295.04 & 7241.1 & 50.46 & 27458 & 0.661 & 1.10 & 0.50 & 99.27 \\
\hline 12.700 & -2.223 & 295.04 & 295.06 & 7234.4 & 50.51 & 27457 & 0.661 & 1.10 & 0.50 & 11.27 \\
\hline 2.540 & 2.197 & 295.04 & 294.97 & 7261.5 & 50.31 & 27461 & 0.661 & 1.10 & 0.50 & 91.27 \\
\hline 5.080 & 2.223 & 295.07 & 294.99 & 7254.7 & 50.37 & 27460 & 0.661 & 1.10 & 0.50 & 19.27 \\
\hline 7.620 & 2.223 & 295.06 & 295.02 & 7247.9 & $50: 41$ & 27459 & 0.661 & 1.10 & 0.50 & 19.27 \\
\hline 10.147 & 2.223 & 295.06 & 295.04 & 7241.2 & 50.46 & 27458 & 0.661 & 1.10 & 0.50 & 19.27 \\
\hline 12.713 & 2.223 & 295.07 & 295.06 & 7234.3 & 50.51 & 27457 & 0.661 & 1.10 & 0.50 & 11.27 \\
\hline 7.607 & -3.493 & 295.01 & 295.02 & 7247.9 & 50.41 & 27459 & 0.661 & 1.10 & 0.50 & 19.27 \\
\hline 7.633 & 3.493 & 295.06 & 295.02 & 7247.9 & 50.42 & 27459 & 0.661 & 1.10 & 0.50 & 11.27 \\
\hline
\end{tabular}


Table 3 (continued)

Channel specimen

Experiment 2

Date: 6 August 1990

rime: $09: 39: 03$

$\begin{array}{cccccccc}\text { TA } & T B & M & P O & P O-P 1 & V f & f & \text { Wf } \\ K & K & \mathrm{~kg} / \mathrm{h} & \mathrm{kPQ} & \mathrm{kPa} & \boldsymbol{X} & & \%\end{array}$

$\begin{array}{llllllll}294.68 & 294.81 & 16.59 & 7326.4 & 8.10 & -0.01 & 0.00537 & 17.20\end{array}$

Hot-side Temperatures:

$\begin{array}{ccc}X & Y & T H \\ \mathrm{~cm} & \mathrm{~cm} & K \\ 1.588 & 0.655 & 294.74 \\ 2.540 & 0.655 & 294.75 \\ 5.080 & 0.655 & 294.72 \\ 7.620 & 0.655 & 294.74 \\ 10.160 & 0.655 & 294.73 \\ 12.700 & 0.655 & 294.75 \\ 13.653 & 0.655 & 294.78\end{array}$

Insulated-Side Temperatures and Calculated Data:

\begin{tabular}{|c|c|c|c|c|c|c|c|c|c|c|}
\hline \multirow[b]{2}{*}{$x$} & \multirow[b]{2}{*}{ Y } & \multirow[b]{2}{*}{$T W$} & \multirow[b]{2}{*}{ If } & \multirow[b]{2}{*}{$\mathbf{P}$} & \multirow[b]{2}{*}{ v } & \multirow[b]{2}{*}{ RE } & \multirow[b]{2}{*}{ PR } & \multicolumn{3}{|c|}{--Uncertainties.- } \\
\hline & & & & & & & & Hew & Wtf & Hre \\
\hline $\mathrm{cm}$ & $\mathrm{cm}$ & $\mathbf{k}$ & $k$ & $\mathrm{kPo}$ & $\mathrm{m} / \mathrm{s}$ & & & $k$ & K & $x$ \\
\hline 0.000 & -0.965 & 294.73 & 294.64 & 7326.4 & 20.15 & 11111 & 0.661 & 1.10 & 0.50 & 11.27 \\
\hline 1.270 & -0.318 & 294.71 & 294.65 & 7325.8 & 20.15 & 11111 & 0.661 & 1.10 & 0.50 & 11.27 \\
\hline 2.527 & -0.330 & 294.71 & 294.66 & 7325.1 & 20.15 & 11110 & 0.661 & 1.10 & 0.50 & 11.27 \\
\hline 3.810 & -0.318 & 294.73 & 294.67 & 7324.4 & 20.16 & 11110 & 0.661 & 1.10 & 0.50 & 11.27 \\
\hline 5.080 & -0.318 & 294.71 & 294.68 & 7323.7 & 20.16 & 11110 & 0.661 & 1.10 & 0.50 & 11.27 \\
\hline 6.363 & -0.318 & 294.71 & 294.69 & 7323.1 & 20.16 & 11110 & 0.661 & 1.10 & 0.50 & 11.27 \\
\hline 7.620 & -0.330 & 294.71 & 294.70 & 7322.4 & 20.16 & 11109 & 0.661 & 1.10 & 0.50 & 11.27 \\
\hline 8.903 & -0.305 & 294.73 & 294.71 & 7321.7 & 20.17 & 11109 & 0.661 & 1.10 & 0.50 & 11.27 \\
\hline 0.160 & -0.318 & 294.72 & 294.72 & 7321.0 & 20.17 & 11109 & 0.661 & 1.10 & 0.50 & 11.27 \\
\hline 11.430 & -0.318 & 294.71 & 294.73 & 7320.4 & 20.17 & 11109 & 0.661 & 1.10 & 0.50 & 11.27 \\
\hline 12.700 & -0.305 & 294.71 & 294.74 & 7319.7 & 20.17 & 11109 & 0.661 & 1.10 & 0.50 & 11.27 \\
\hline 13.970 & -0.318 & 294.73 & 294.75 & 7319.0 & 20.18 & 11108 & 0.661 & 1.10 & 0.50 & 11.27 \\
\hline 15.240 & -0.953 & 294.74 & 294.76 & 7318.3 & 20.18 & 11108 & 0.661 & 1.10 & 0.50 & 11.27 \\
\hline 2.527 & -2.223 & 294.70 & 294.66 & & 20.15 & 11110 & 0.661 & 1.10 & 0.50 & 11.27 \\
\hline 5.080 & -2.235 & 294.80 & 294.68 & 7323.7 & 20.16 & 11110 & 0.661 & 1.10 & 0.50 & 11.27 \\
\hline 7.607 & & 294.72 & 294.70 & 7322.4 & 20.16 & 11109 & 0.661 & 1.10 & 0.50 & 11.27 \\
\hline 10.173 & -2.223 & 294.72 & 294.72 & 7321.0 & 20.17 & 11109 & 0.661 & 1.10 & 0.50 & 19.27 \\
\hline 12.700 & -2.223 & 294.68 & 294.74 & 7319.7 & 20.17 & 11109 & 0.661 & 1.10 & 0.50 & 19.27 \\
\hline 2.540 & 2.197 & 294.76 & 294.66 & 7325.1 & 20.15 & 11110 & 0.661 & 1.10 & 0.50 & 11.27 \\
\hline 5.080 & 2.223 & 294.74 & 294.68 & 7323.7 & 20.16 & 11110 & 0.661 & 1.10 & 0.50 & 11.27 \\
\hline 7.620 & 2.223 & 294.74 & 294.70 & 7322.4 & 20.16 & 11109 & 0.661 & 1.10 & 0.50 & 11.27 \\
\hline 10.147 & 2.223 & 294.73 & 294.72 & 7321.0 & 20.17 & 11109 & 0.661 & 1.10 & 0.50 & 11.27 \\
\hline 12.713 & 2.223 & 294.75 & 294.74 & 7319.7 & 20.17 & 11109 & 0.661 & 1.10 & 0.50 & 11.27 \\
\hline 7.607 & -3.493 & 294.71 & 294.70 & 7322.4 & 20.16 & 11109 & 0.661 & 1.10 & 0.50 & 11.27 \\
\hline 7.633 & 3.493 & 294.76 & 294.70 & 7322.4 & 20.16 & 11109 & 0.661 & 1.10 & 0.50 & 19.27 \\
\hline
\end{tabular}




\section{Table 3 (continued)}

Channel Specimen

Experiment 2

Date: 6 August 1990

$T$ ime: $09: 42: 20$

$\begin{array}{cccccccc}T A & T B & M & P O & P 0-P 1 & V F & f & H f \\ K & K & k g / h & K P a & K P a & \% & & \% \\ 294.62 & 294.74 & 7.78 & 7343.6 & 2.03 & -0.01 & 0.00641 & 18.40\end{array}$

Hot-side Temperatures:

$\begin{array}{lll}x & Y & T H\end{array}$

cm cm $\quad K$

$\begin{array}{lll}1.588 & 0.655 & 294.67\end{array}$

$\begin{array}{llll}2.540 & 0.655 & 294.68\end{array}$

$\begin{array}{llll}5.080 & 0.655 & 294.68\end{array}$

$\begin{array}{llll}7.620 & 0.655 & 294.70\end{array}$

$\begin{array}{llll}10.160 & 0.655 & 294.68\end{array}$

$\begin{array}{llll}12.700 & 0.655 & 294.67\end{array}$

$\begin{array}{lll}13.653 & 0.655 \quad 294.69\end{array}$

Insulated-Side Temperatures and Calculated Data:

\begin{tabular}{|c|c|c|c|c|c|c|c|c|c|c|}
\hline & & & & & & & & & & \\
\hline$x$ & $Y$ & $\tau_{H}$ & If & 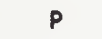 & v & RE & PR & tw & Hef & Wre \\
\hline $\mathrm{cm}$ & $\mathrm{cm}$ & 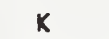 & $\mathrm{K}$ & $\mathrm{kPa}$ & $\mathrm{m} / \mathrm{s}$ & & & $\mathrm{K}$ & K & $x$ \\
\hline 0.000 & -0.965 & 294.65 & 294.61 & 7343.6 & 9.22 & 5098 & 0.661 & 1.10 & 0.50 & 11.27 \\
\hline 1.270 & -0.318 & 294.66 & 294.62 & 7343.4 & 9.22 & 5098 & 0.661 & 1.10 & 0.50 & 11.27 \\
\hline 2.527 & -0.330 & 294.66 & 294.63 & 7343.3 & 9.23 & 5098 & 0.661 & 1.10 & 0.50 & 11.27 \\
\hline 3.810 & -0.318 & 294.69 & 294.64 & 7343.1 & 9.23 & 5098 & 0.661 & 1.10 & 0.50 & 11.27 \\
\hline 5.080 & -0.318 & 294.66 & 294.65 & 7342.9 & 9.23 & 5098 & 0.661 & 1.10 & 0.50 & 11.27 \\
\hline 6.363 & -0.318 & 294.67 & 294.66 & 7342.8 & 9.23 & 5098 & 0.661 & 1.10 & 0.50 & 11.27 \\
\hline 7.620 & -0.330 & 294.66 & 294.67 & 7362.6 & 9.23 & 5098 & 0.661 & 1.10 & 0.50 & 11.27 \\
\hline 8.903 & -0.305 & 294.66 & 294.68 & 7342.4 & 9.23 & 5098 & 0.661 & 1.10 & 0.50 & 11.27 \\
\hline 0.160 & -0.318 & 294.67 & 294.69 & 7342.2 & 9.23 & 5098 & 0.661 & 1.10 & 0.50 & 11.27 \\
\hline 11.430 & -0.318 & 294.64 & 294.70 & 7342.1 & 9.23 & 5097 & 0.661 & 1.10 & 0.50 & 11.27 \\
\hline 12.700 & -0.305 & 294.66 & 294.71 & 7341.9 & 9.23 & 5097 & 0.661 & 1.10 & 0.50 & 11.27 \\
\hline 13.970 & -0.318 & 294.67 & 294.72 & 7341.7 & 9.23 & 5097 & 0.661 & 1.10 & 0.50 & 11.27 \\
\hline 15.240 & -0.953 & 294.67 & 294.73 & 7341.6 & 9.23 & 5097 & 0.661 & 1.10 & 0.50 & 11.27 \\
\hline 2.527 & -2.223 & 294.66 & 294.63 & 7343.3 & 9.23 & 5098 & 0.661 & 1.10 & 0.50 & 11.27 \\
\hline 5.080 & -2.235 & 294.71 & 294.65 & 7342.9 & 9.23 & 5098 & 0.661 & 1.10 & 0.50 & 11.27 \\
\hline 7.607 & -2.235 & 294.67 & 294.67 & 7342.6 & 9.23 & 5098 & 0.661 & 1.10 & 0.50 & 11.27 \\
\hline 10.173 & -2.223 & 294.67 & 294.69 & 7342.2 & 9.23 & 5098 & 0.661 & 1.10 & 0.50 & 11.27 \\
\hline 12.700 & -2.223 & 294.66 & 294.71 & 7341.9 & 9.23 & 5097 & 0.661 & 1.10 & 0.50 & 11.27 \\
\hline 2.540 & 2.197 & 294.68 & 294.63 & 7343.3 & 9.23 & 5098 & 0.661 & 9.10 & 0.50 & 11.27 \\
\hline 5.080 & 2.223 & 294.69 & 294.65 & 7342.9 & 9.23 & 5098 & 0.661 & 1.10 & 0.50 & 11.27 \\
\hline 7.620 & 2.223 & 294.70 & 294.67 & 7342.6 & 9.23 & 5098 & 0.661 & 1.10 & 0.50 & 11.27 \\
\hline 10.147 & 2.223 & 294.68 & 294.69 & 7342.3 & 9.23 & 5098 & 0.661 & 1.10 & 0.50 & 11.27 \\
\hline 12.713 & 2.223 & 294.70 & 294.71 & 7341.9 & 9.23 & 5097 & 0.661 & 1.10 & 0.50 & 11.27 \\
\hline 7.607 & -3.493 & 294.64 & 294.67 & 7342.6 & 9.23 & 5098 & 0.661 & 1.10 & 0.50 & 11.27 \\
\hline 7.633 & 3.493 & 294.70 & 294.67 & 7342.6 & 9.23 & 5098 & 0.661 & 1.10 & 0.50 & 11.27 \\
\hline
\end{tabular}


Table 3 (continued)

Channel specimen

Experiment 3

Date: 6 August 1990

Time: $15: 01: 28$

$\begin{array}{cccccccc}\text { TA } & T B & M & P O & P 0-P 1 & \text { Vf } & \text { Ot } & \text { Hqt } \\ K & K & \mathrm{~kg} / \mathrm{h} & \mathrm{KPa} & \mathrm{kPa} & \mathrm{X} & \mathrm{H} & \mathrm{X}\end{array}$

$\begin{array}{llllllll}299.70 & 699.35 & 4.06 & 3443.6 & 3.92 & 26.13 & 2342.0 & 1.04\end{array}$

$\begin{array}{ccc}X & Y & T W \\ \mathrm{~cm} & \mathrm{~cm} & K \\ 1.588 & 0.655 & 484.71 \\ 2.540 & 0.655 & 519.83 \\ 5.080 & 0.655 & 599.49 \\ 7.620 & 0.655 & 669.22 \\ 10.160 & 0.655 & 727.07 \\ 12.700 & 0.655 & 769.69 \\ 13.653 & 0.655 & 778.98\end{array}$

Insulated-side Temperatures and Calculated Data:

\begin{tabular}{|c|c|c|c|c|c|c|c|c|c|c|c|c|c|c|c|c|}
\hline$x$ & $Y$ & $T H$ & Tf & Taw. & P & $v$ & RE & $R$ & h & NU & NUm & tw & itf & Hre & Wh & Wnu \\
\hline $\mathrm{cm}$ & $\mathrm{cm}$ & $k$ & $k$ & $k$ & $\mathrm{kPa}$ & $\mathrm{m} / \mathrm{s}$ & & & $H /\left(m^{2} \cdot k\right)$ & & & K & $k$ & \% & $\%$ & $\%$ \\
\hline 0.000 & -0.965 & 87.89 & 299.70 & 299.70 & 3443.6 & 10.06 & 2597 & 0.665 & 1552 & 9.44 & 10.88 & 1.10 & 2.16 & 1.27 & 11.60 & 12.60 \\
\hline 1.270 & -0.318 & 55.51 & 333.01 & 333.02 & 3443.3 & 11.15 & 2418 & 0.665 & 1531 & 8.67 & 10.30 & 1.10 & 3.47 & 1.27 & 5.00 & 7.02 \\
\hline 3.810 & -0.318 & 47.83 & 397.94 & 397.95 & 3442.7 & 13.30 & 2141 & 0.666 & 1109 & 5.56 & 6.63 & 1.10 & 6.12 & 1.27 & 6.06 & 7.81 \\
\hline 5.080 & -0.318 & 585.29 & 429.63 & 429.65 & 3442.3 & 14.34 & 2031 & 0.666 & 1097 & 5.22 & 6.19 & 1.25 & 7.80 & 1.27 & 6.67 & 8.29 \\
\hline 6.363 & -0.318 & 621.45 & 462.33 & 462.35 & 3442.0 & 15.42 & 1931 & 0.666 & 1085 & 4.91 & 5.78 & 1.39 & 9.57 & 1.27 & 7.43 & 8.91 \\
\hline 8.903 & -0.305 & 685.24 & 527.18 & 527.21 & 3441.4 & 17.56 & 1763 & 0.666 & 1088 & 4.50 & 5.20 & 1.65 & 12.71 & 11.27 & 9.18 & 10.41 \\
\hline 10.160 & -0.318 & 713.25 & 559.03 & 559.05 & 3441.0 & 18.62 & 1693 & 0.666 & 1098 & 4.36 & 4.99 & 1.76 & 14.15 & 11.27 & 10.22 & 11.35 \\
\hline 11.430 & -0.318 & 736.90 & 590.41 & 590.44 & 3440.7 & 19.65 & 1630 & 0.666 & 1122 & 4.29 & 4.85 & 1.86 & 15.64 & 11.27 & 11.65 & 12.64 \\
\hline 12.700 & -0.305 & 757.29 & 621.38 & 621.42 & 3440.4 & 20.68 & 1573 & 0.666 & 1234 & 4.55 & 5.07 & 1.94 & 17.18 & 11.27 & 13.47 & 14.35 \\
\hline 13.970 & -0.318 & 767.11 & 654.51 & 654.55 & 3440.0 & 21.77 & 1517 & 0.666 & 1640 & 5.83 & 6.36 & 1.98 & 18.91 & 11.27 & 17.37 & 18.05 \\
\hline 15.240 & -0.953 & 754.41 & 686.86 & 686.90 & 3439.7 & 22.84 & 1466 & 0.666 & 1897 & 6.52 & 6.87 & 1.92 & 20.48 & 11.27 & 33.00 & 33.37 \\
\hline 7.607 & -2.235 & 664.29 & 499.68 & 499.70 & 3441.7 & 16.19 & 1779 & 0.666 & 1047 & 4.49 & 5.25 & 1.57 & 11.30 & 11.27 & 8.15 & 9.52 \\
\hline 10.173 & -2.223 & 727.11 & 566.78 & 566.80 & 3441.0 & 18.35 & 1630 & 0.666 & 1056 & 4.15 & 4.76 & 1.82 & 14.17 & 11.27 & 9.92 & 11.07 \\
\hline 12.700 & -2.223 & 770.75 & 630.58 & 630.62 & 3440.4 & 20.40 & 1513 & 0.666 & 1196 & 4.36 & 4.87 & 1.99 & 17.18 & 1.27 & 13.11 & 14.01 \\
\hline 2.540 & 2.197 & 513.52 & 369.02 & 369.03 & 3443.0 & 11.91 & $21 \pi$ & 0.666 & 1175 & 6.20 & 7.44 & 1.10 & 4.60 & 11.27 & 5.47 & 7.36 \\
\hline 5.080 & 2.223 & 595.89 & 434.37 & 434.39 & 3442.3 & 13.89 & 1945 & 0.666 & 1057 & 4.99 & 5.94 & 1.29 & 7.80 & 11.27 & 6.53 & 8.18 \\
\hline 7.620 & 2.223 & 667.40 & 501.55 & 501.58 & 3441.7 & 16.13 & 1761 & 0.666 & 1039 & 4.45 & 5.21 & 1.58 & 11.32 & 11.27 & 8.12 & 9.49 \\
\hline 10.147 & 2.223 & 725.17 & 568.14 & 568.17 & 3441.0 & 18.25 & 1615 & 0.666 & 1079 & 4.23 & 4.84 & 1.81 & 14.14 & 11.27 & 10.07 & 11.21 \\
\hline 12.713 & 2.223 & 766.30 & 633.45 & 633.48 & 3440.4 & 20.33 & 1497 & 0.666 & 1263 & 4.59 & 5.10 & 1.97 & 17.20 & 11.27 & 13.77 & 14.62 \\
\hline 7.607 & -3.493 & 672.38 & 504.12 & 504.14 & 3441.7 & 15.98 & 1730 & 0.666 & 1024 & 4.37 & 5.12 & 1.60 & 11.30 & 11.27 & 8.02 & 9.41 \\
\hline 7.633 & 3.493 & 683.86 & 510.78 & 510.80 & 3441.7 & 15.73 & 1666 & 0.666 & 996 & 4.21 & 4.94 & 1.64 & 11.34 & 11.27 & 7.88 & 9.29 \\
\hline
\end{tabular}


Channel specimen

Experiment 3

Date: 6 August 1990

rime: 15:10:52

\begin{tabular}{|c|c|c|c|c|c|c|c|}
\hline TA & TB & M & PO & $P 0=P 1$ & Vf & at & Hat \\
\hline $\mathbf{K}$ & $\mathbf{K}$ & $\mathrm{kg} / \mathrm{h}$ & KPa & KPo & $x$ & $H$ & $x$ \\
\hline 298.61 & 513.76 & 8.41 & 3437.8 & 8.59 & 26.13 & 2608.0 & 1.07 \\
\hline
\end{tabular}

Hot-side Temperatures:

$\begin{array}{ccc}X & Y & T W \\ \mathrm{CD} & \mathrm{CN} & K \\ 1.588 & 0.655 & 404.89 \\ 2.540 & 0.655 & 420.92 \\ 5.080 & 0.655 & 458.73 \\ 7.620 & 0.655 & 497.67 \\ 10.160 & 0.655 & 534.74 \\ 12.700 & 0.655 & 551.69 \\ 13.653 & 0.655 & 557.13\end{array}$

Insulated-side Temperatures and Calculated Dota:

\begin{tabular}{|c|c|c|c|c|c|c|c|c|c|c|c|c|c|c|c|c|}
\hline $\begin{array}{r}x \\
\mathrm{~cm}\end{array}$ & $\begin{array}{r}Y \\
-\quad c m\end{array}$ & $\begin{array}{r}T H \\
K\end{array}$ & $\begin{array}{l}\text { if } \\
k\end{array}$ & $\begin{array}{c}\text { Taw } \\
K\end{array}$ & $\begin{array}{c}P \\
k P o\end{array}$ & $\begin{array}{c}v \\
m / s\end{array}$ & $R E$ & PR & $\begin{array}{c}h \\
H /\left(m^{2} \cdot K\right)\end{array}$ & MU & MUm & $\begin{array}{c}\text { Wtw } \\
K\end{array}$ & $\begin{array}{l}\text { Wtf } \\
K\end{array}$ & $\begin{array}{c}\text { Wre } \\
x\end{array}$ & $\begin{array}{l}\text { Wh } \\
x\end{array}$ & $\begin{array}{c}\text { Whu } \\
\%\end{array}$ \\
\hline 0.000 & -0.965 & 44.89 & 298.57 & 298.61 & 3437.8 & 21.26 & 5515 & 0.665 & 3295 & 20.08 & 21.74 & 1.10 & 1.24 & 11.27 & 11.83 & 12.81 \\
\hline 1.270 & -0.318 & 84.19 & 316.08 & 16.12 & 3437.1 & 22.49 & 5307 & 0.665 & 3070 & 18.01 & 20.05 & 1.10 & 1.91 & 11.27 & 5.17 & 7.14 \\
\hline 2.527 & -0.330 & 408.25 & 333.56 & 333.61 & 3436.4 & 23.72 & 5117 & 0.665 & 2535 & 14.34 & 16.03 & 1.10 & 2.49 & 11.27 & 5.71 & 7.54 \\
\hline 3.810 & -0.318 & 428.63 & 350.21 & 350.27 & 3435.7 & 24.89 & 4950 & 0.665 & 2363 & 12.93 & 14.45 & 1.10 & 3.31 & 11.27 & 6.28 & 7.98 \\
\hline 5.080 & -0.318 & 446.82 & 366.88 & 366.93 & 3434.9 & 26.06 & 4795 & 0.666 & 2380 & 12.62 & 14.07 & 1.10 & 4.21 & 11.27 & 6.96 & 8.53 \\
\hline 6.363 & -0.318 & 465.42 & 384.06 & 384.12 & 3434.2 & 27.26 & 4648 & 0.666 & 2365 & 12.16 & 13.52 & 1.10 & 5.16 & 1.27 & 7.78 & 9.20 \\
\hline 7.620 & -0.330 & 484.05 & 400.95 & 401.02 & 3433.5 & 28.45 & 4513 & 0.666 & 23 & 11.54 & 12.80 & 1.10 & 6.10 & & 8.61 & 9.92 \\
\hline 8.903 & -0.305 & 503.79 & 18.15 & 418.23 & & & 4384 & 0.666 & 22 & 10.86 & 12.03 & 1.10 & 6.85 & & & .41 \\
\hline 0.160 & -0.318 & 519.72 & 434.89 & 434.97 & & & 6267 & 0.666 & & 10.51 & 11.59 & & 7.63 & & & 1.22 \\
\hline 11.430 & -0.318 & 532.90 & 451.39 & 451.47 & & & 4159 & 0.666 & 22 & 10.34 & 11.33 & & 8.43 & & 36 & 12.38 \\
\hline 12.700 & -0.305 & 542.24 & 467.67 & 467.76 & & & 4059 & 0.666 & & 11.26 & 12.21 & & 9.26 & & & 14.16 \\
\hline 13.970 & -0.318 & 47.46 & 485.08 & 485.18 & & & 3958 & 0.666 & & 14.46 & 15.45 & & & & & .65 \\
\hline 15.240 & -0.953 & 536.48 & 502.08 & 502.19 & 342 & & 3864 & 0.666 & 4160 & 17.79 & 18.45 & & .03 & 27 & 73 & 35.08 \\
\hline 5.080 & -2.235 & 459.30 & 370.36 & 370.41 & 34 & 25.03 & 4534 & 0.666 & & 11.27 & 12.69 & & 4.21 & & 6.54 & 8.18 \\
\hline 7.607 & -2.235 & 493.32 & 405.99 & 406.05 & $34 ?$ & 27.41 & 4257 & 0.666 & 2199 & 10.89 & 12.12 & 0 & 6.09 & & 8.29 & 9.64 \\
\hline 10.173 & -2.223 & 527.78 & 442.02 & 442.10 & 343 & 29.8 & 4015 & 0.666 & 2201 & 10.28 & 11.33 & & 7.63 & & 00 & 11.15 \\
\hline 12.700 & -2.223 & 551.80 & 476.29 & 476.38 & 3430.6 & 32.1 & 3814 & 0.666 & 24 & 10.98 & 11.91 & & 9.26 & & & 14.03 \\
\hline 2.540 & 2.197 & 414.37 & 336.04 & 336.08 & 34 & 22.42 & 4778 & 0.665 & & 13.59 & & & 2.50 & & & 7.46 \\
\hline 5.080 & 2.223 & 454.92 & 371.36 & 371.41 & & 24.75 & 4463 & 0.666 & & & & & & & 6.78 & 8.38 \\
\hline 7.620 & 2.223 & 496.21 & 407.67 & 407.73 & & & 4187 & 0.666 & & 10.71 & 11.93 & & 6.10 & & 8.22 & 9.58 \\
\hline 10.147 & 2.223 & 530.94 & 443.66 & 443.73 & 3432.1 & 29.52 & 3950 & 0.666 & 2164 & 10.08 & 11.13 & 1.10 & 7.62 & 11.27 & 9.84 & 11.01 \\
\hline 12.713 & 2.223 & 550.08 & 478.95 & 479.03 & 3430.6 & 31.86 & 3747 & 0.666 & 2630 & 11.62 & 12.54 & 1.11 & 9.27 & 11.27 & 13.86 & 14.71 \\
\hline 7.607 & -3.493 & 501.51 & 410.50 & 410.56 & 3433.5 & 26.59 & 4055 & 0.666 & 2111 & 10.37 & 11.58 & 1.10 & 6.09 & 11.27 & 8.05 & 9.43 \\
\hline 7.633 & 3.493 & 510.99 & 415.94 & 415.99 & 3433.5 & 25.78 & 3845 & 0.666 & 2021 & 9.84 & 11.02 & 1.10 & 6.11 & 11.27 & 7.82 & 9.24 \\
\hline
\end{tabular}




\section{Table 3 (continued)}

Channel Specimen

Experiment 3

Date: 6 August 1990

Time: 15:17:37

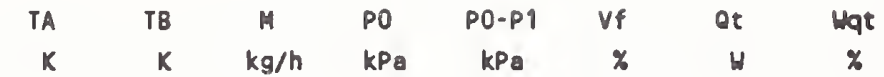

$\begin{array}{llllllll}297.87 & 419.79 & 14.94 & 3441.3 & 20.86 & 26.14 & 2625.0 & 1.17\end{array}$

Hot-side Temperatures:

$\begin{array}{ccc}X & Y & T H \\ \mathrm{~cm} & \mathrm{~cm} & K \\ 1.588 & 0.655 & 364.10 \\ 2.540 & 0.655 & 372.15 \\ 5.080 & 0.655 & 392.15 \\ 7.620 & 0.655 & 413.26 \\ 10.160 & 0.655 & 429.28 \\ 12.700 & 0.655 & 443.74 \\ 13.653 & 0.655 & 450.48\end{array}$

Insulated-Side Temperatures and Calculated Data:

\begin{tabular}{|c|c|c|c|c|c|c|c|c|c|c|c|c|c|c|c|c|}
\hline$x$ & $Y$ & $T w$ & Tf & Taw & $\mathbf{P}$ & $v$ & $\mathbf{F}$ & PR & h & NU & ML & Wtw & Wtf & Wre & Wh & Wnu \\
\hline $\mathrm{cm}$ & $\mathrm{cm}$ & K & K & K & $\mathrm{KPa}$ & $\mathrm{m} / \mathrm{s}$ & & & $H /\left(\mathbb{A}^{2} \cdot\right.$ & & & K & K & $x$ & $x$ & $\%$ \\
\hline 0.000 & -0.965 & 22.86 & 297.73 & 97.86 & 3441.3 & 3.31 & 9995 & .665 & 6140 & 37.48 & 39.19 & 1.10 & 0.81 & 11.27 & .54 & .47 \\
\hline 1.270 & -0.318 & 347.35 & 307.47 & 07.60 & 3439.5 & .56 & 9782 & .665 & 291 & 31.62 & 3.81 & 0 & 1.15 & 7 & 5.70 & .53 \\
\hline 2.527 & -0.330 & 360.31 & 317.19 & 317.33 & 3437.8 & 0.81 & 9578 & .665 & 431 & 25.93 & 7.81 & 10 & 1.46 & 7 & 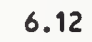 & 7.86 \\
\hline 3.810 & -0.318 & 71.12 & 26.45 & 26.60 & 43 & 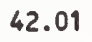 & 393 & .665 & 186 & 24.03 & 5.79 & 0 & 1.90 & 7 & 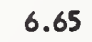 & 8.27 \\
\hline .080 & -0.318 & 30.62 & 5.71 & 35.87 & 3434.3 & 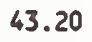 & 216 & .665 & 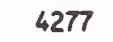 & 27.0 & 81 & 0 & .41 & 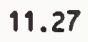 & & 8.84 \\
\hline 363 & 318 & 57 & .27 & 45.43 & 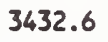 & $\checkmark$ & 342 & 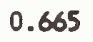 & . & 2 & 36 & 0 & .94 & 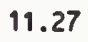 & & 9.55 \\
\hline 620 & 0 & 7 & 84.66 & 83 & 1308 & 565 & 378 & 5 & 4225 & 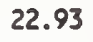 & ? & & 7 & & & .31 \\
\hline 903 & 5 & 6 & 34 & 1 & acy. & 898 & 719 & 6 & 4142 & 22.07 & & & 3.89 & & & .88 \\
\hline 0 & 8 & 83 & 3 & 2 & & & 570 & 6 & 4305 & 0 & 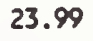 & & 3 & & & .09 \\
\hline .430 & 18 & 34 & 70 & 82.90 & & & ? & • & 4342 & 22.37 & 1 & & 9 & & & 36 \\
\hline .700 & -0.305 & 26 & 1.75 & 91.97 & & & 5 & 66 & & 08 & 24.07 & & 6 & & & .60 \\
\hline .970 & -0.318 & 40.02 & 01.43 & 01.66 & 3422.1 & 31.8 & 8157 & 66 & 5396 & 2 & 28.30 & & 8 & & & 6.63 \\
\hline .240 & -0.953 & 95 & 10.89 & 11.12 & 3420.4 & .14 & 8028 & .666 & 2081 & 30. & 6 & & 6 & & & 31.01 \\
\hline 2.527 & -2 & 38 & 18.65 & 18.77 & 3437.8 & 3.18 & 891 & .665 & in & 6 & s. & & 6 & & & 7.73 \\
\hline 5.080 & -2.2 & 60 & 38.55 & 38.68 & 3434.3 & 40.30 & 533 & 665 & 3549 & 0 & 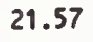 & 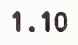 & 1 & & & 8.20 \\
\hline 7.607 & -2.235 & 28.07 & 58.79 & 58.95 & 430. & .9 & 8202 & .665 & 3933 & 8 & 2. & 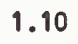 & .46 & & 7 & 9.88 \\
\hline 0.173 & -2.223 & 425.35 & 379.27 & 379.44 & 3427. & 5.46 & 7897 & 0.666 & 13 & 21.44 & 2.84 & 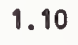 & .34 & 27 & & 11.77 \\
\hline 2.700 & -2.223 & 441.04 & 398.74 & 398.93 & 3423.9 & 47.80 & 7631 & 0.666 & 4463 & 22.36 & 3.63 & 0 & 5.26 & 27 & & 14.38 \\
\hline 2.540 & 2.197 & 364.73 & 318.75 & 18.87 & 3437.8 & 8.19 & 8889 & 0.665 & 4149 & 24.20 & 26.06 & 1.10 & 1.46 & 11.27 & & 7.72 \\
\hline 5.080 & 2.223 & 386.84 & 338.54 & 338.68 & 3434.3 & 40.56 & 8533 & 0.665 & 3974 & 22.26 & 23.95 & 1.10 & 2.41 & 11.27 & 7.01 & 8.57 \\
\hline 7.620 & 2.223 & 408.15 & 358.89 & 359.05 & 3430.8 & 3.01 & 8201 & 0.665 & 3934 & 21.18 & 2.73 & 10 & 3.47 & 11.27 & 58 & 9.89 \\
\hline 0.147 & 2.223 & 424.62 & 379.06 & 79.23 & 3427.4 & 5.43 & 7900 & 0.666 & 184 & 21.70 & 3.10 & 0 & .33 & 27 & 77 & 11.84 \\
\hline 2.713 & 2.223 & 440.54 & 398.83 & $\$ .02$ & 3423 & 2 & 7630 & 0.666 & 530 & 69 & 3.97 & 0 & 26 & 11.27 & 13.70 & 14.55 \\
\hline 7.607 & -3.493 & 417 & 364.10 & 64.23 & 3430.9 & 5 & 7473 & 0.665 & 0 & 1 & .81 & & 3.46 & 11.27 & 8.06 & 9.45 \\
\hline 7.633 & .493 & 19.11 & 5.06 & 65.19 & 430.8 & 39.81 & 7379 & .666 & 3583 & 07 & 0.57 & 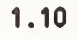 & 3.47 & 11.27 & 8.02 & 9.41 \\
\hline
\end{tabular}




\section{Table 3 (continued)}

Channel Specimen

Experiment 3

Date: 6 August 1990

rime: 15:22:37

$\begin{array}{cccccccc}T A & T B & M & P O & P O-P 1 & V f & \text { at } & \text { Wqt } \\ K & K & k g / h & K P B & K P g & * & H & \% \\ 297.80 & 387.89 & 20.00 & 3447.3 & 33.13 & 26.15 & 2597.0 & 1.29\end{array}$

$\begin{array}{ccc}X & Y & T W \\ \mathrm{~cm} & \mathrm{~cm} & K \\ 1.588 & 0.655 & 351.35 \\ 2.540 & 0.655 & 356.94 \\ 5.080 & 0.655 & 371.58 \\ 7.620 & 0.655 & 387.22 \\ 10.160 & 0.655 & 399.16 \\ 12.700 & 0.655 & 410.28 \\ 13.653 & 0.655 & 415.78\end{array}$

Insulated-Side Temperatures and Calculated Data:

\begin{tabular}{|c|c|c|c|c|c|c|c|c|c|c|c|c|c|c|c|c|}
\hline $\begin{array}{c}x \\
c m\end{array}$ & $\begin{array}{c}Y \\
\mathrm{~cm}\end{array}$ & $\begin{array}{l}\text { Tw } \\
K\end{array}$ & $\begin{array}{r}T f \\
K\end{array}$ & $\begin{array}{l}\text { Taw } \\
K\end{array}$ & ${ }_{\mathrm{KPa}}^{\mathrm{P}}$ & $\begin{array}{c}v \\
\mathrm{~m} / \mathrm{s}\end{array}$ & RE & PR & $\begin{array}{c}h \\
H /\left(m^{2} \cdot k\right)\end{array}$ & NU & $\lim$ & $\begin{array}{c}\text { Wtw } \\
K\end{array}$ & $\begin{array}{c}\text { Wtf } \\
K\end{array}$ & $\begin{array}{c}\text { Wre } \\
x\end{array}$ & th & $\begin{array}{l}\text { Wnu } \\
\%\end{array}$ \\
\hline 0.000 & .965 & 16.30 & 97.55 & 297.77 & 647.3 & 51.66 & 13514 & 0.665 & 8194 & 50.06 & 1.75 & 1.10 & 0.69 & 11.27 & 13.29 & 14.17 \\
\hline .270 & .318 & 5.98 & 24.67 & 24.91 & 44.5 & 2.92 & 13301 & .665 & 6695 & 40.26 & 2.49 & .10 & 0.90 & .27 & 6.14 & 7.87 \\
\hline 2.527 & .330 & 5.50 & 1.78 & 2.03 & 41.8 & . 17 & 13096 & .665 & 228 & .32 & 5.26 & .10 & 1.11 & .27 & 5.45 & 8.11 \\
\hline .810 & .318 & 3.49 & 18.55 & 8.81 & 39.0 & 37 & 12907 & .665 & 17 & .03 & 2.86 & 10 & .43 & 1.27 & 5.87 & 8.45 \\
\hline 5.080 & -0.318 & 60.42 & 325.33 & 325.60 & 36.2 & 6.58 & 12724 & .665 & 36 & 31.28 & 3.09 & 1.10 & 1.80 & 1.27 & 7.47 & 8.95 \\
\hline .363 & -0.318 & 367.73 & 332.32 & 332.60 & 33.4 & 82 & 12542 & .665 & 50 & 30.91 & 2.68 & 1.10 & 2.18 & .27 & 8.21 & 9.57 \\
\hline 7.620 & -0.330 & 375.12 & 339.18 & 339.48 & 30.7 & 9.04 & 12369 & 0.665 & 363 & 30.00 & 1.71 & 1.10 & 2.58 & 1.27 & 3.98 & 0.24 \\
\hline 3.903 & -0.305 & 82.95 & 346.18 & 46.49 & .9 & 60.29 & 12198 & .665 & 30 & 28.85 & 0.50 & 10 & 2.89 & 1.27 & 9.53 & 10.72 \\
\hline .160 & -0.318 & 387.99 & 352.99 & 353.31 & 2 & 69.50 & 12037 & 0.665 & 15 & 29.48 & 31.05 & 1.10 & 3.21 & 1.27 & 10.74 & 11.81 \\
\hline .430 & -0.318 & 393.77 & 359.70 & 360.03 & 22.4 & .71 & 11884 & .665 & 5400 & 29.03 & 0.51 & 1.10 & 3.55 & .27 & .90 & 2.88 \\
\hline .700 & -0.305 & 399.84 & 366.32 & 66.66 & 419.7 & 63.89 & 11737 & 0.666 & 302 & 29.74 & 21 & 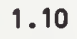 & 3.89 & .27 & .00 & 13.90 \\
\hline .970 & -0.318 & 05.37 & 373.39 & 373.75 & y & 16 & 11585 & 66 & 78 & 33.95 & 52 & 0 & 4.28 & 27 & .59 & 5.40 \\
\hline 15.240 & -0.953 & 400.15 & 380.31 & 380.68 & .1 & 41 & 11440 & 66 & 95 & 37.76 & 8.83 & 10 & 4.64 & 27 & .59 & 28.03 \\
\hline 2.527 & -2.223 & 348.65 & 313.06 & 313.27 & 441.8 & 03 & 12012 & 0.665 & 25 & 4 & 36 & 0 & 1.11 & 27 & .27 & 7.97 \\
\hline 5.080 & -2.235 & 11.72 & 327.80 & 328.03 & 436.2 & .43 & 11643 & .665 & 4334 & 24.81 & 26.59 & 1.10 & 1.80 & .27 & .52 & 8.17 \\
\hline 7.607 & -2.235 & 381.79 & 342.79 & 343.05 & .7 & .87 & 11295 & 0.665 & 4933 & 27.40 & 29.07 & 1.1 & 2.57 & .27 & 8.43 & 9.76 \\
\hline 0.173 & -2.223 & 394.29 & 357.95 & 358.23 & .2 & 35 & 10967 & 0.665 & 07 & 28.08 & 61 & 1.10 & 3.21 & .27 & 0.41 & 11.51 \\
\hline 12.700 & -2.223 & 406.38 & 372.37 & 372.67 & 419.7 & 59.73 & $106 \sqrt{5}$ & 0.666 & 15 & 28.96 & 39 & 1.10 & 3.89 & .27 & 12.82 & 13.73 \\
\hline 2.540 & 2.197 & 348.98 & 312.95 & 313.17 & 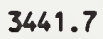 & 61 & 12159 & 0.665 & 5256 & 31 & 96 & 1.10 & 1.12 & 1.27 & 6.24 & 7.94 \\
\hline 5.080 & 2.223 & 365.33 & 327.43 & 32 & .2 & .00 & 11792 & .665 & 5027 & 28.80 & .59 & 1.10 & 1.80 & .27 & 7.11 & 8.65 \\
\hline 7.620 & 2.223 & 380.86 & 342.33 & 342.58 & & 55.46 & 11441 & 0.665 & 3 & 27.76 & 29.44 & 1.10 & 2.58 & .27 & .51 & 9.83 \\
\hline 0.147 & 2.223 & 393.73 & 357.08 & 357.36 & 425.2 & 57.90 & 11117 & 0.665 & 67 & 27.91 & 29.45 & 1.10 & 3.21 & 1.27 & 0.32 & 11.43 \\
\hline 2.713 & 2.223 & 405.93 & 371.55 & 371.86 & 419.6 & 60.31 & 10820 & 0.666 & 5460 & 28.71 & 30.14 & 1.10 & 3.90 & 11.27 & 12.71 & 13.63 \\
\hline 7.607 & -3.493 & & 348.16 & & & & 10002 & 665 & & 24.26 & & . & 2.57 & 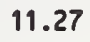 & 7.79 & 9.21 \\
\hline 7.633 & 3.493 & 390.45 & 347.60 & 347.81 & 3430.7 & 50.51 & 10158 & 0.665 & 4482 & 24.66 & 26.29 & 1.10 & 2.58 & 11.27 & 7.88 & 9.29 \\
\hline
\end{tabular}


Table 3 (continued)

Channel specimen

Experiment 3

Date: 6 August 1990

Time: $15: 27: 56$

$\begin{array}{cccccccc}T A & T B & M & P O & P O-P 1 & V F & \text { Ot } & \text { Wqt } \\ K & K & \mathrm{Kg} / \mathrm{h} & \mathrm{KPa} & \mathrm{KPa} & \mathrm{K} & \mathrm{H} & \mathbf{X} \\ 297.58 & 358.53 & 29.39 & 3449.5 & 62.41 & 26.17 & 2580.0 & 1.55\end{array}$

Hot-side Temperatures:

$\begin{array}{ccc}X & Y & T w \\ \mathrm{~cm} & \mathrm{~cm} & K \\ 1.588 & 0.655 & 338.81 \\ 2.540 & 0.655 & 342.26 \\ 5.080 & 0.655 & 352.06 \\ 7.620 & 0.655 & 362.66 \\ 10.160 & 0.655 & 370.67 \\ 12.700 & 0.655 & 378.30 \\ 13.653 & 0.655 & 382.52\end{array}$

Insulated-Side Temperatures and Calculated Data:

\begin{tabular}{|c|c|c|c|c|c|c|c|c|c|c|c|c|c|c|c|c|}
\hline $\begin{array}{c}x \\
c m\end{array}$ & $\begin{array}{c}Y \\
\mathrm{~cm}\end{array}$ & $\begin{array}{l}\text { Tw } \\
K\end{array}$ & $\begin{array}{r}T f \\
k\end{array}$ & $\begin{array}{c}\text { Taw } \\
K\end{array}$ & $\begin{array}{l}P \\
k P g\end{array}$ & $\begin{array}{c}v \\
\mathrm{~m} / \mathrm{s}\end{array}$ & RE & PR & h & NU & NUm & $\begin{array}{l}\text { Wew } \\
k\end{array}$ & $\begin{array}{l}\text { def } \\
K\end{array}$ & $\begin{array}{c}\text { Wre } \\
\%\end{array}$ & $\begin{array}{l}\text { Wh } \\
\%\end{array}$ & $\%$ \\
\hline 000 & & 10.12 & 7.01 & 97.51 & & $76 . \pi 7$ & 155 & & 11959 & 2 & .88 & & 0.59 & 1.27 & 5.05 & \\
\hline 70 & & & & & & & 43 & & & & 9 & & & & 12 & \\
\hline .527 & .330 & 1.26 & 6.49 & 7.02 & 39 & .41 & 9735 & 65 & & .24 & 8.26 & & .81 & 27 & 24 & .7 \\
\hline 810 & .31 & 9 & 9 & & & & 441 & & & & & & & & & \\
\hline 080 & .318 & .27 & .51 & .07 & & 95 & 352 & 65 & 164 & 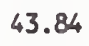 & 7 & & 25 & 27 & 97 & \\
\hline 363 & 18 & 15 & 6 & & & & 61 & & & & & & & & & 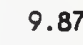 \\
\hline .620 & 0.33 & & & & & & & & & & & & 76 & & 18 & 0.4 \\
\hline .903 & .30 & 6.68 & 8 & 0. & & & 18796 & & & 2 & 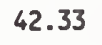 & & 97 & & 55 & 0.7 \\
\hline 160 & 318 & 11 & 1 & & & & 23 & & & & & & & & & 1. \\
\hline 430 & 318 & 3 & 38.38 & & & & 456 & & & & & & & & 66 & 2. \\
\hline .700 & 305 & 92 & 342.78 & & & & 3294 & & & & 0 & & & & 60 & 3. \\
\hline .970 & .318 & 2.16 & 7.49 & 8.19 & & 8 & 8125 & 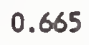 & & & 2 & & 92 & & 68 & 4.5 \\
\hline .240 & .953 & & & & & & 17963 & & & & & & & & & 5. \\
\hline 2.527 & & & 307. & 12 & & & 822 & & & & & & & & &. \\
\hline 5.080 & 2.235 & 2.09 & 17.56 & 8.03 & & .66 & 7442 & 66 & & 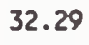 & .18 & & 25 & & 62 & .2 \\
\hline 7.607 & 2.235 & 5.70 & 327. & 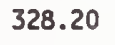 & & & 75 & & & 5 & 7 & & 76 & & 52 & \\
\hline .173 & 2.223 & & & & & & & & & & & & & & & 1. \\
\hline 2.700 & -2.223 & 2.83 & 7.71 & 8.28 & & & 16401 & & & & 10 & & & & 12.56 & 13. \\
\hline 2.540 & 2.1 & & & & & & 3270 & & & & & & & & & \\
\hline 5.080 & 2.223 & & & & & & 391 & & & & 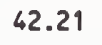 & & & & 7.55 & \\
\hline 7.620 & & & & & & & & & & & & & & & & \\
\hline .147 & 2.223 & 4.18 & 36.85 & 37.41 & & & 17171 & & & 3 & .91 & & .19 & 27 & .19 & 11. \\
\hline .713 & & & & & & & 44 & & & & & & 65 & & 12.14 & 13. \\
\hline & & & & & & & & & & & & & & & 66 & \\
\hline & & & & & & & & & & & & & 76 & 27 & .93 & \\
\hline
\end{tabular}


Table 3 (continued)

Channel specimen

Experiment 3

Date: 6 August 1990

Time: 15:32:39

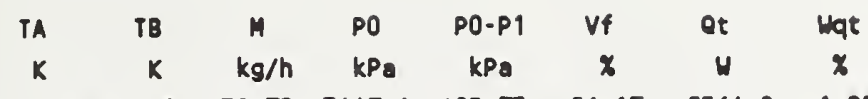

$\begin{array}{llllllll}297.08 & 341.84 & 39.79 & 3447.6 & 105.75 & 26.17 & 2564.0 & 1.88\end{array}$

Hot-side Temperatures:

$\begin{array}{ccc}X & Y & T W \\ \mathrm{~cm} & \mathrm{~cm} & K \\ 1.588 & 0.655 & 331.08 \\ 2.540 & 0.655 & 333.33 \\ 5.080 & 0.655 & 340.55 \\ 7.620 & 0.655 & 348.32 \\ 10.160 & 0.655 & 354.08 \\ 12.700 & 0.655 & 359.61 \\ 13.653 & 0.655 & 363.11\end{array}$

Insulated-side Temperatures and Calculated Data:

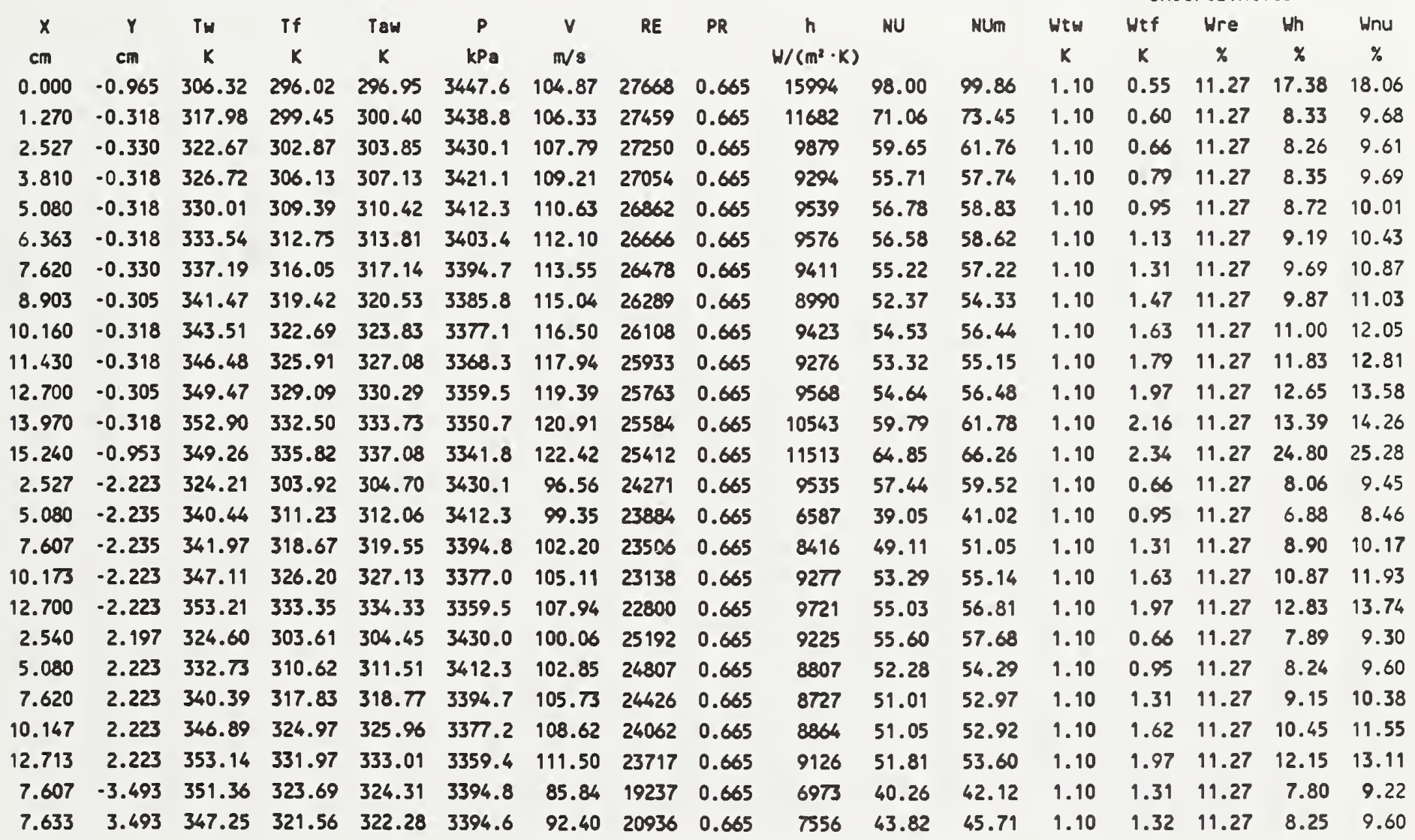


Table 3 (continued)

Channel specimen

Experiment 4

Date: 20 July 1990

rime: 15:04:08

$\begin{array}{cccccccc}T A & T B & M & P O & P 0-P 1 & V f & \text { Ot } & \text { Hat } \\ K & K & K g / h & K P a & K P a & \% & W & \% \\ 295.67 & 634.88 & 10.16 & 3595.6 & 14.28 & 49.93 & 4970.0 & 1.04\end{array}$

Hot-side Temperatures:

$\begin{array}{ccc}x & Y & T H \\ \mathrm{~cm} & \mathrm{~cm} & K \\ 1.588 & 0.655 & 477.48 \\ 2.540 & 0.655 & 504.02 \\ 5.080 & 0.655 & 564.52 \\ 7.620 & 0.655 & 629.08 \\ 10.160 & 0.655 & 693.42 \\ 12.700 & 0.655 & 726.55 \\ 13.653 & 0.655 & 732.35\end{array}$

Insulated-Side Temperatures and Calculated Data:

\begin{tabular}{|c|c|c|c|c|c|c|c|c|c|c|c|c|c|c|c|c|}
\hline $\begin{array}{l}x \\
\mathrm{~cm}\end{array}$ & $\begin{array}{c}r \\
\mathrm{~cm}\end{array}$ & Tw & $\begin{array}{r}\text { Tf } \\
K\end{array}$ & $\begin{array}{l}\text { Taw } \\
K\end{array}$ & $\begin{array}{c}P \\
k P a\end{array}$ & $\begin{array}{c}V \\
m / 8\end{array}$ & RE & PR & $\begin{array}{c}h \\
W /\left(m^{2} \cdot K\right)\end{array}$ & NU & Im & Wtw & $\begin{array}{l}\text { Wtf } \\
K\end{array}$ & $\begin{array}{l}\text { Hre } \\
x\end{array}$ & Wh & $\begin{array}{l}\text { Hnu } \\
\%\end{array}$ \\
\hline .000 & .965 & 70.84 & 95.61 & 95.66 & 595.6 & 24.60 & 6779 & 665 & 3865 & 23.69 & . & 1.10 & 1.86 & .27 & 1.63 & 12.63 \\
\hline 0 & 18 & 77 & 2.93 & 2.99 & 44 & 84 & 3387 & 665 & 39 & .87 & .52 & .10 & .96 & & 4.86 & 6.92 \\
\hline 2.527 & 0.330 & 9.63 & 50.19 & 350.26 & 993.2 & 29.08 & 6045 & 0.665 & 2787 & 15.25 & 18.13 & 1.10 & 3.90 & 1.27 & 5.38 & 7.29 \\
\hline 3.810 & -0.318 & 11.87 & 376.17 & 376.25 & 992.0 & .21 & 5757 & 0.665 & 2602 & 13.56 & 6.06 & 1.10 & 5.20 & 27 & 5.91 & 7.69 \\
\hline 5.080 & -0.318 & 0.81 & 402.15 & 402.25 & 9 & 35 & 5500 & 666 & 2615 & 02 & .32 & & 6.63 & & 6.50 & 8.15 \\
\hline 6.363 & -0.318 & 570.66 & 428.96 & 429.07 & 89.6 & .55 & 5261 & .666 & 2587 & 12.33 & .43 & .19 & 8.13 & 27 & 7.21 & 8.73 \\
\hline 7.620 & -0.330 & 601.10 & 55.30 & 455.42 & 388.5 & .72 & 5050 & 0.666 & 2511 & 11.48 & 13.38 & 1.31 & 9.61 & .27 & 7.92 & 9.32 \\
\hline 8.903 & -0.305 & 633.89 & 482.14 & 482.27 & 887.3 & 39.93 & 4854 & 0.666 & 407 & 10.58 & 12.30 & 1.44 & 10.79 & 1.27 & 8.37 & 9.70 \\
\hline .160 & -0.318 & 662.23 & 508.24 & 508.39 & 1 & 42.08 & 4680 & 0.666 & 2337 & 9.90 & 11.45 & 1.56 & 2.02 & 27 & 9.00 & 10.26 \\
\hline .430 & -0.318 & 86.38 & 533.97 & 34.13 & .9 & .20 & 4522 & 0.666 & 2291 & 38 & .77 & 5 & 3.28 & 27 & 9.86 & 11.02 \\
\hline 2.700 & -0.305 & 4.59 & 59.36 & 559.54 & .7 & 46.30 & 4379 & 0.666 & 2453 & 9 & 11.05 & 1.73 & 14.59 & .27 & 11.05 & 12.10 \\
\hline 3.970 & -0.318 & 12.79 & 586.51 & 586.71 & 882.5 & 48.54 & 4237 & 0.666 & 3108 & 11.93 & 13.28 & 1.76 & 16.05 & 1.27 & 13.44 & 14.31 \\
\hline 15.240 & -0.953 & 689.08 & 613.03 & 613.25 & 3 & & 4108 & & 3585 & 13.34 & 14.23 & & 17.38 & & 26.29 & 26.74 \\
\hline 2.527 & -2.223 & 493.96 & 354.07 & 354.13 & 3593 & 27 & 5603 & 0.665 & & 14 & 16.81 & & 3.90 & & 5.25 & 7.20 \\
\hline 5.080 & -2.235 & 560.70 & 409.72 & 409.80 & 590 & 31.72 & 5071 & 0.666 & 24 & 1.8 & 14.02 & 1. & 6.63 & $? 7$ & 6.21 & 7.92 \\
\hline 7.607 & -2.235 & 622.45 & 466.34 & 466.45 & .5 & 36.07 & 4638 & 0.666 & 2345 & 10.55 & 12.37 & 1.40 & 9.60 & 27 & 7.55 & 9.01 \\
\hline 10.173 & -2.223 & 680.92 & 523.61 & $523 . \pi 3$ & 886.1 & 40.47 & 4281 & 0.666 & 2287 & 9.49 & 10.97 & 1.63 & 12.03 & 27 & 8.87 & 10.14 \\
\hline 12.700 & -2.223 & 726.45 & 578.07 & 578.24 & 3583.7 & 44.67 & 3996 & 0.666 & 2400 & 9.30 & 10.55 & 1.81 & 14.59 & 11.27 & 10.86 & 11.92 \\
\hline 2.540 & 2.197 & 488.17 & 354.01 & 354.08 & 3593. & 27.61 & 5636 & 0.665 & 2687 & 14.59 & 17.41 & 1. & 3.91 & 27 & 5.33 & 7.25 \\
\hline 5.080 & 2.223 & 551.94 & 409.06 & 409.15 & r & 31 & 5106 & 0.666 & 2537 & 12.49 & 14.73 & 1. & 6.63 & 27 & 6.39 & 8.07 \\
\hline 7.620 & 2.223 & 620.86 & 465.65 & 465.76 & 3588.5 & 36.22 & 4670 & 0.666 & 2358 & 10.62 & 12.44 & 1.39 & 9.61 & 11.27 & 7.59 & 9.04 \\
\hline 10.147 & 2.223 & 683.42 & 521.73 & 521.87 & 3586.1 & 40.56 & 4316 & 0.666 & 2226 & 9.26 & 10.74 & 1.64 & 12.00 & 11.27 & 8.67 & 9.97 \\
\hline 12.713 & 2.223 & 720.26 & 576.73 & 576.90 & 3583 & 44.82 & 4026 & 0.666 & 483 & 9.64 & 10.89 & 1.79 & 14.60 & 11.27 & 11.17 & 12.21 \\
\hline 7.607 & -3.493 & 43.02 & 78.14 & 478.24 & & & 4265 & 0.000 & 21 & 9. & 4 & & 9.60 & & 7.23 & 8.74 \\
\hline 7.633 & 3.493 & 648.99 & 480.53 & 480.63 & 3588.5 & 34.42 & 4209 & 0.666 & 2172 & 9.57 & 11.29 & 1.50 & 9.62 & 11.27 & 7.20 & 8.72 \\
\hline
\end{tabular}


Channel Specimen

Experiment 4

Date: 20 July 1990

Time: $15: 11: 13$

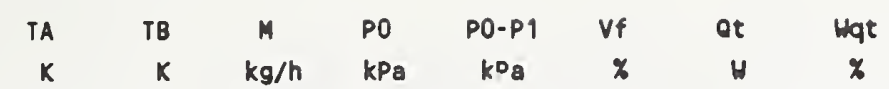

$\begin{array}{llllllll}295.25 & 533.98 & 14.90 & 3591.4 & 24.47 & 49.94 & 5128.0 & 1.06\end{array}$

Hot-side Temperatures:

$\begin{array}{ccc}X & Y & T H \\ \mathrm{~cm} & \mathrm{~cm} & K \\ 1.588 & 0.655 & 428.33 \\ 2.540 & 0.655 & 444.66 \\ 5.080 & 0.655 & 484.10 \\ 7.620 & 0.655 & 526.70 \\ 10.160 & 0.655 & 558.49 \\ 12.700 & 0.655 & 586.03 \\ 13.653 & 0.655 & 600.64\end{array}$

Insulated-side Temperatures and Calculated Data:

\begin{tabular}{|c|c|c|c|c|c|c|c|c|c|c|c|c|c|c|c|c|}
\hline & & & & & & & & & & & & & & & & \\
\hline$x$ & $Y$ & $T H$ & Tf & Тан & $P$ & $v$ & RE & $R$ & h & NU & NUm & Hew & Hef & Wre & Wh & Wnu \\
\hline $\mathrm{cm}$ & $\mathrm{cm}$ & $K$ & K & K & kPa & $\mathrm{m} / \mathrm{s}$ & & & $H /\left(m^{2} \cdot K\right)$ & & & K & $\mathbf{K}$ & $x$ & \% & $\%$ \\
\hline 0.000 & -0.965 & 45.55 & 95.13 & 75.24 & 3591.4 & 36.22 & 9995 & 0.665 & 5960 & 36.57 & 39.88 & 1.10 & 1.35 & 11.27 & 11.79 & 12.78 \\
\hline 1.270 & -0.318 & 94.50 & 14.26 & 314.38 & 3589.4 & 38.55 & 583 & .665 & 5127 & 30.18 & 34.20 & .10 & 2.11 & 1.27 & 5.00 & 7.02 \\
\hline 2.527 & -0.330 & 420.84 & 333.35 & 333.49 & 3587.3 & 40.87 & 9207 & 0.665 & 4259 & 24.09 & 27.38 & 1.10 & 2.76 & 1.27 & 5.55 & 7.42 \\
\hline 3.810 & -0.318 & 441.74 & 351.54 & 351.69 & 3585.3 & 43.09 & 8881 & .665 & 4043 & 22.07 & 25.02 & .10 & 3.67 & 11.27 & 6.14 & 7.87 \\
\hline 5.080 & -0.318 & 460.55 & 369.73 & 369.90 & 3583.3 & 45.31 & 8580 & .665 & 4124 & 21.7 & 24.54 & .10 & 4.67 & 1.27 & 6.84 & 8.42 \\
\hline 6.363 & -0.318 & 480.14 & 388.50 & 388.69 & 3581.2 & 47.60 & 3295 & .665 & 134 & 21.08 & 3.68 & - & 5.72 & 27 & 7.68 & 9.12 \\
\hline 7.620 & -0.330 & 00.16 & 406.94 & 407.15 & 3579.2 & 49.8 & 3035 & .666 & 4058 & 20 & 2.45 & 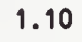 & 6.77 & 7 & 8.53 & 9.85 \\
\hline 8.903 & -0.305 & 19.96 & 425.73 & 425.96 & 3577.1 & 2.1 & 790 & .666 & 006 & ? & 21.42 & 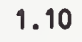 & 7.60 & 27 & .23 & 0.46 \\
\hline 0.160 & -0.318 & 34.08 & 444.01 & 444.26 & 35 & 54. & 7568 & .666 & 4129 & 19.21 & 2 & 1.10 & 8.46 & 27 & .45 & 1.55 \\
\hline 11.430 & -0.318 & 47.98 & 462.02 & 462.29 & 3573.1 & 56. & 7363 & .666 & 4200 & 1 & 20.88 & 0 & 9.35 & .27 & 11.86 & 12.84 \\
\hline 12.700 & -0.305 & 563.60 & 479.80 & 480.09 & 3571.0 & 58.7 & 7174 & 0.666 & 4396 & 19 & 21.19 & 1.16 & 10.27 & 11.27 & 13.15 & 14.04 \\
\hline 13.970 & -0.318 & 577.54 & 498.81 & 499.12 & 3569.0 & 61.13 & 6983 & 0.666 & 5157 & 22.14 & 24.00 & 1.22 & 11.30 & 11.27 & 15.05 & 15.84 \\
\hline 15.240 & -0.953 & 565.42 & 517.38 & 517.71 & 3566.9 & 63.42 & 6809 & 0.666 & 5879 & 24.61 & 25.84 & 1.17 & 12.24 & 11.27 & 28.72 & 29.14 \\
\hline 5.080 & -2.235 & 477.31 & 374.77 & 374.92 & 3583.3 & 43.03 & 7967 & 0.665 & 3651 & 19.08 & 21.79 & 1.10 & 4.67 & 11.27 & 6.38 & 8.06 \\
\hline 7.607 & -2.235 & 513.74 & 414.28 & 414.47 & 3579.2 & 47.55 & 7438 & 0.666 & 3802 & 18.55 & 20.88 & 1.10 & 6.76 & 11.27 & 8.12 & 9.50 \\
\hline 10.173 & -2.223 & 550.71 & 454.24 & 454.47 & 3575.1 & 52.14 & 6981 & 0.666 & 3853 & 17.65 & 19.62 & 1.11 & 8.47 & 11.27 & 9.89 & 11.04 \\
\hline 12.700 & -2.223 & 580.20 & 492.24 & 492.51 & 3571.0 & 56.51 & 6604 & 0.666 & 4186 & 18.14 & 19.86 & 1.23 & 10.27 & 11.27 & 12.60 & 13.53 \\
\hline 2.540 & 2.197 & 429.06 & 336.56 & 336.68 & 3587.3 & 38.26 & 8484 & 0.665 & 4024 & 22.62 & 25.85 & 1.10 & 2.77 & 11.27 & 5.44 & 7.34 \\
\hline 5.080 & 2.223 & 471.91 & 375.60 & 375.75 & 3583.3 & 42.68 & 7873 & 0.665 & 3888 & 20.28 & 22.99 & 1.10 & 4.67 & 11.27 & 6.61 & 8.24 \\
\hline 7.620 & 2.223 & 516.19 & 415.72 & 415.91 & 3579.2 & 47.22 & 7344 & 0.666 & 3764 & 18.32 & 20.64 & 1.10 & 6.77 & 11.27 & 8.07 & 9.45 \\
\hline 10.147 & 2.223 & 546.75 & 455.49 & 455.71 & $35 \sqrt{5} .1$ & 51.74 & 6896 & 0.666 & 4075 & 18.63 & 20.60 & 1.10 & 8.45 & 11.27 & 10.33 & 11.44 \\
\hline 12.713 & 2.223 & 578.00 & 494.49 & 494.75 & 3571.0 & 56.18 & 6516 & 0.666 & 4413 & 19.06 & 20.77 & 1.22 & 10.28 & 11.27 & 13.20 & 14.09 \\
\hline 7.607 & $\cdot 3.493$ & 527.91 & 422.02 & 422.20 & 3579.2 & 45.48 & 6897 & 0.666 & 3570 & 17.20 & 19.45 & 1.10 & 6.76 & 1.27 & 7.77 & 9.20 \\
\hline 7.633 & 3.493 & 538.30 & 427.95 & 428.12 & 3579.1 & 44.21 & 6549 & 0.666 & 3425 & 16.35 & 18.55 & 1.10 & 6.78 & 11.27 & 7.57 & 9.02 \\
\hline
\end{tabular}


Channel Specimen

Experiment 4

Date: 20 July 1990

Time: $15: 16: 44$

$\begin{array}{cccccccc}\text { TA } & T B & M & P O & P 0-P 1 & V f & \text { Qt } & \text { Wat } \\ K & K & \mathrm{~kg} / \mathrm{h} & \mathrm{kPa} & \mathrm{kPa} & \mathrm{X} & \mathrm{H} & \mathrm{X} \\ 294.88 & 470.79 & 20.34 & 3585.6 & 38.35 & 49.96 & 5158.0 & 1.10\end{array}$

Hot-side Temperatures:

$\begin{array}{ccc}X & Y & T H \\ \mathrm{~cm} & \mathrm{~cm} & K \\ 1.588 & 0.655 & 400.79 \\ 2.540 & 0.655 & 411.90 \\ 5.080 & 0.655 & 440.42 \\ 7.620 & 0.655 & 471.15 \\ 10.160 & 0.655 & 494.35 \\ 12.700 & 0.655 & 516.23 \\ 13.653 & 0.655 & 527.78\end{array}$

Insulated-Side Temperatures and Calculated Data:

\begin{tabular}{|c|c|c|c|c|c|c|c|c|c|c|c|c|c|c|c|c|}
\hline & & & & & & & & & & & & & & & & \\
\hline$x$ & $Y$ & $T_{H}$ & $f$ & aw & $\mathbf{P}$ & $v$ & E & $R$ & " & IU & Num & $w$ & itf & Wre & Wh & Whu \\
\hline $\mathrm{cm}$ & $\mathrm{cm}$ & $K$ & $k$ & $K$ & $\mathrm{kPa}$ & $\mathrm{n} / \mathrm{s}$ & & & $/\left(m^{2} \cdot k\right)$ & & & $x$ & $k$ & $x$ & $x$ & $\%$ \\
\hline 0.000 & 0.965 & 31.79 & 94.64 & 94.85 & 585.6 & 49.84 & 3769 & .665 & 8163 & 0.15 & 3.53 & 1.10 & 1.05 & 1.27 & 12.00 & 12.97 \\
\hline 1.270 & 0.318 & 70.04 & 08.61 & 08.84 & 582.4 & 2.20 & 13348 & .665 & 751 & 0.23 & 4.45 & 1.10 & 1.58 & 1.27 & 5.12 & 7.10 \\
\hline 2.527 & 0.330 & 88.94 & 22.55 & 322.80 & 3579.3 & 4.57 & 2955 & .665 & 657 & 32.73 & 36.28 & 1.10 & 2.05 & 1.27 & 5.63 & 7.48 \\
\hline 3.810 & 0.318 & 04.09 & 35.84 & 336.11 & 576.0 & 6.83 & 12605 & .665 & 387 & 0.33 & 3.58 & 1.10 & 2.72 & 1.27 & 6.19 & 7.90 \\
\hline 5.080 & 0.318 & 17.68 & 49.13 & 349.42 & 572.8 & 9.09 & $122 \pi$ & 0.665 & 509 & 0.21 & 3.34 & .10 & 3.45 & 1.27 & 6.85 & 8.44 \\
\hline 6.363 & -0.318 & 31.88 & 362.84 & 363.16 & 569.6 & 1.43 & 11959 & 0.665 & 533 & 9.56 & 32.53 & .10 & 4.22 & 1.27 & 7.67 & 9.11 \\
\hline 7.620 & -0.330 & 46.21 & 376.31 & 376.65 & 566.5 & .73 & 11665 & 0.665 & 457 & 28.44 & 31.23 & 1.10 & 4.99 & 1.27 & 8.52 & 9.83 \\
\hline 8.903 & -0.305 & 60.51 & 390.03 & 390.40 & 563.2 & 6.08 & 11383 & 0.665 & 403 & 27.47 & 30.10 & .10 & 5.60 & .27 & 9.21 & 10.44 \\
\hline .160 & -0.318 & 471.05 & 403.38 & 403.77 & 3560.1 & 8.37 & 11124 & 0.666 & 5545 & 7.56 & 30.01 & .10 & 6.24 & .27 & 0.38 & 11.49 \\
\hline .430 & -0.318 & 482.22 & 416.53 & 416.95 & 3556.9 & 0.63 & 10881 & 0.666 & 5546 & 26.96 & 29.22 & .10 & 6.89 & .27 & 1.60 & 12.60 \\
\hline .700 & -0.305 & 494.29 & 429.52 & 429.96 & 3553.7 & 2.87 & 10654 & 0.666 & 739 & 7.32 & 29.51 & & 7.57 & & 2.70 & 13.62 \\
\hline .970 & -0.318 & 505.54 & 443.40 & 443.88 & 3550.5 & .26 & 10423 & 0.666 & 26 & 30.72 & 33.02 & & 8.33 & & 14.23 & 15.05 \\
\hline 5.240 & .0 .953 & 95.29 & 456.96 & 457.46 & & .61 & 10209 & 0.666 & & 34.02 & 35.56 & & 9.02 & & 27.17 & 27.61 \\
\hline 2.527 & -2.223 & 96.32 & 324.88 & 325.10 & 9.3 & .79 & 11916 & 0.665 & & 30.24 & 33.73 & & 2.05 & 27 & 5.48 & 7.36 \\
\hline 5.080 & -2.235 & 433.96 & 353.64 & 353.90 & 象 & .31 & 11249 & 0.665 & 7 & 25.53 & 28.57 & 10 & 3.45 & 27 & 6.27 & 7.97 \\
\hline 7.607 & -2.235 & 58.48 & 382.90 & 383.20 & 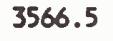 & .92 & 10655 & 0.665 & 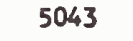 & 20.81 & 28.67 & 10 & 4.99 & 11.27 & 8.03 & 9.42 \\
\hline .173 & -2.223 & 84.44 & 412.50 & 412.85 & 0.0 & .61 & 10125 & 0.666 & 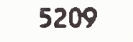 & 25.50 & 27.86 & & 6.24 & 11.27 & 9.88 & 11.03 \\
\hline 12.700 & -2.223 & 508.39 & 440.63 & 441.04 & (1) & 9.07 & 9676 & 0.666 & & 25.64 & 27.74 & & 7.57 & 11.27 & 12.20 & 13.15 \\
\hline 2.540 & 2.197 & 395.75 & 324.81 & 325.03 & (2) & 1.14 & 12003 & 0.665 & & 30.44 & 33.93 & 10 & 2.06 & 11.27 & 5.50 & 7.38 \\
\hline 5.080 & 2.223 & 427.08 & 353.22 & 353.48 & 20.0. & 5.64 & 11338 & 0.665 & > & 28.78 & 30.85 & 1.0 & 3.45 & 11.27 & 6.56 & 8.20 \\
\hline 7.620 & 2.223 & 457.46 & 382.43 & 382.73 & 3566.5 & 60.28 & 10740 & 0.665 & 280 & 26.18 & 28.89 & 1.10 & 4.99 & 11.27 & 8.08 & 9.46 \\
\hline 0.147 & 2.223 & 482.54 & 411.37 & 411.72 & 3560.1 & 64.89 & 10216 & 0.666 & 3209 & 25.84 & 28.21 & 10 & 6.23 & 11.27 & 9.95 & 11.10 \\
\hline 12.713 & 2.223 & 506.78 & 439.75 & 440.15 & 3553.6 & 69.43 & 9758 & 0.666 & 5545 & 25.97 & 28.08 & 1.10 & 7.58 & 11.27 & 12.32 & 13.27 \\
\hline 7.607 & -3.493 & 473.50 & 391.06 & 391.32 & 3566.5 & 56.04 & 9619 & 0.665 & 4619 & 23.45 & 26.05 & 1.10 & 4.99 & 11.27 & 7.55 & 9.02 \\
\hline 7.633 & 3.493 & 476.21 & 392.71 & 392.97 & 3566.4 & 55.52 & 9463 & 0.666 & 4560 & 23.08 & 25.66 & 1.10 & 5.00 & 11.27 & 7.50 & 8.97 \\
\hline
\end{tabular}




\section{Table 3 (continued)}

Chamel specimen

Experiment 4

Date: 20 July 1990

Time: $15: 21: 44$

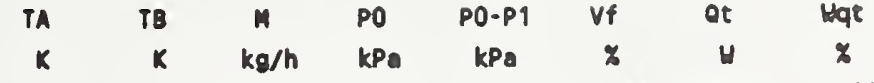

$\begin{array}{llllllll}294.49 & 412.50 & 30.30 & 3567.6 & 70.45 & 49.98 & 5152.0 & 1.18\end{array}$

Hot-side Temperatures:

$\begin{array}{ccc}X & Y & T w \\ \mathrm{~cm} & \mathrm{~cm} & K \\ 1.588 & 0.655 & 375.14 \\ 2.540 & 0.655 & 381.82 \\ 5.080 & 0.655 & 400.85 \\ 7.620 & 0.655 & 421.43 \\ 10.160 & 0.655 & 437.13 \\ 12.700 & 0.655 & 452.10 \\ 13.653 & 0.655 & 460.76\end{array}$

Insulated-Side Temperatures and Calculated Data:

\begin{tabular}{|c|c|c|c|c|c|c|c|c|c|c|c|c|c|c|c|c|}
\hline $\mathrm{cm}$ & $\mathrm{cm}$ & $\begin{array}{c}T_{W} \\
K\end{array}$ & $k$ & $\begin{array}{c}\text { Taw } \\
\text { K }\end{array}$ & $\mathrm{kPa}$ & $\mathrm{m} / \mathrm{s}$ & & & $H /\left(m^{2} \cdot k\right)$ & 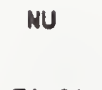 & & K & $\mathrm{K}$ & $\begin{array}{c}\text { Wre } \\
x\end{array}$ & $x$ & $\%$ \\
\hline 000 & .965 & 9.46 & 3.95 & 4.42 & 67.6 & 31 & 20784 & .665 & 12034 & 74.04 & 7. & 1.10 & 0.80 & .27 & 2.52 & 5.4 \\
\hline 270 & 318 & 7.51 & 3.19 & 3.70 & .8 & & 0360 & 665 & & .80 & & & & & 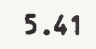 & 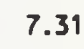 \\
\hline 27 & & 9.99 & & 95 & & & 9952 & 665 & & .96 & 7 & & 1 & & & \\
\hline & & & & & & & & & & & & & & & & .5 \\
\hline .080 & .3 & 2 & 9.99 & 9 & 9 & & 19227 & .665 & & . & 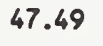 & & .33 & .27 & 86 & .4 \\
\hline 363 & & 0 & 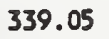 & & & & 18876 & 665 & & & & & & & & . $C$ \\
\hline 20 & & 8.14 & 96 & & & & 8546 & 65 & & & & & 36 & & & . \\
\hline & & & & & & & & & & & & & & & & .1 \\
\hline 160 & & & 6 & & & & & & & & & & 19 & & & 1.1 \\
\hline 43 & & 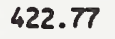 & 4 & & & & 17638 & & & & 1 & & 63 & & & 2.0 \\
\hline 700 & & 4 & 14 & & & & 17 & & & & & & & & & 3.9 \\
\hline 97 & & 9.49 & 2. & & & & 7088 & .006 & & & & & 60 & & & 3.8 \\
\hline .24 & .953 & 0.90 & 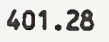 & & & & 16825 & 0.666 & & 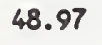 & & & 06 & & & 5.4 \\
\hline .527 & .223 & 2 & & & & & 18094 & & & & & & 11 & & & .5 \\
\hline 08 & & 4.89 & 54 & & & & 17 & & & & & & & & & . \\
\hline 607 & & 8.2 & 3.29 & & & & 16715 & 0.665 & & & & & 35 & & & 9.2 \\
\hline .173 & 223 & 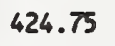 & 3.16 & & & & 1690 & (65 & & & & & 20 & & & .7 \\
\hline .700 & & & & & & & & & & & & & 09 & & & 12.6 \\
\hline .540 & 2.197 & 5.08 & 314.05 & & & & 18424 & 0.665 & & & & & 42 & & & 7.5 \\
\hline .080 & 2.223 & 6. & 2. & & & & 10 & 0.665 & & & & & & & & . \\
\hline 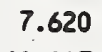 & & & 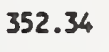 & & & & 17041 & 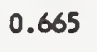 & & & & & 3.36 & & -5 & 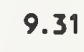 \\
\hline .14 & & & & & & & & & & & & & 4.19 & & & 0. \\
\hline .713 & 223 & 440.56 & & & & & 15888 & & & & & & .09 & 27 & 11.45 & 12. \\
\hline 7.607 & .493 & 1.45 & 360.31 & 360 & & & 14776 & 0.665 & & & .58 & & .35 & & 25 & 8. \\
\hline .633 & 3.493 & 421.97 & 60.72 & 361.20 & 3532.3 & 75.67 & 14724 & 0.665 & 6239 & 33.46 & 36.47 & 1.10 & 3.36 & 1.27 & 7.25 & \\
\hline
\end{tabular}


Table 3 (continued)

Channel specimen

Experiment 4

Date: 20 July 1990

Time: $15: 26: 54$

$\begin{array}{cccccccc}T A & T B & M & P O & P 0-P 1 & V f & \text { ot } & \text { Hqt } \\ K & K & \mathrm{~kg} / \mathrm{h} & \mathrm{kPa} & \mathrm{kPa} & \mathrm{\gamma} & \mathrm{H} & \mathrm{x}\end{array}$

$\begin{array}{llllllll}294.23 & 382.70 & 40.04 & 3550.7 & 111.52 & 49.98 & 5103.0 & 1.30\end{array}$
Hot-side Temperatures:

$\begin{array}{ccc}X & Y & T H \\ \mathrm{~cm} & \mathrm{~cm} & K \\ 1.588 & 0.655 & 361.63 \\ 2.540 & 0.655 & 366.18 \\ 5.080 & 0.655 & 380.59 \\ 7.620 & 0.655 & 396.23 \\ 10.160 & 0.655 & 407.95 \\ 12.700 & 0.655 & 419.17 \\ 13.653 & 0.655 & 426.33\end{array}$

Insulated-side Temperatures and Calculated Data:

\begin{tabular}{|c|c|c|c|c|c|c|c|c|c|c|c|c|c|c|c|c|}
\hline $\mathrm{cm}$ & $\mathrm{cm}$ & $\begin{array}{l}T w \\
K\end{array}$ & K & $\begin{array}{l}\text { Taw } \\
\text { K }\end{array}$ & $\begin{array}{c}\mathrm{P} \\
\mathrm{KPa}\end{array}$ & $\mathrm{m} / \mathrm{s}$ & & R & $\mathrm{H} /\left(\mathrm{m}^{2} \cdot \mathrm{K}\right)$ & U U & $19 \mathrm{~m}$ & Kew & $\begin{array}{l}\text { tf } \\
K\end{array}$ & $\begin{array}{c}\text { Wre } \\
\%\end{array}$ & $\begin{array}{l}\text { wh } \\
\text { x }\end{array}$ & $\%$ \\
\hline 000 & .965 & 3.23 & 3.2 & 1 & .7 & 0.62 & 747 & 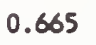 & 15606 & 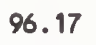 & 99.72 & 1.10 & 0.68 & & 3.17 & \\
\hline 70 & & .73 & .11 & & & & 328 & 665 & & & .03 & & .89 & 27 & 5.78 & \\
\hline & & 4 & & & & & & & & & & & & & & \\
\hline 810 & & 6 & & & & & 537 & & & & & & .41 & .27 & .46 & \\
\hline .080 & .318 & 9.50 & 19.95 & 0.99 & & 5 & 6171 & 665 & 9659 & & 1 & & .76 & & 6.96 & \\
\hline 363 & & 6.53 & 7 & & & & 806 & & & & & & 14 & & 7.59 & \\
\hline 320 & & .73 & .26 & & & & 688 & 35 & & & 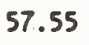 & & 53 & 27 & 8.25 & \\
\hline 90 & & & & & & & & & & & & & & & 8.69 & \\
\hline 16 & 8 & 6. & 1 & & & & 793 & & & & & & 15 & & 70 & . \\
\hline 43 & & 2.38 & $?$ & & & & 4485 & & & & & & & & & 1. \\
\hline 70 & & 9 & 0 & & & & & & & & & & & & & 2.6 \\
\hline 970 & & 5. & 6.10 & & & & 882 & & & & & & & & & 3. \\
\hline .24 & 0 & 8 & 2.73 & & & & 1 & & & & & & 56 & & & 3.8 \\
\hline 527 & & & 3 & & & & & & & & & & & & 5.94 & \\
\hline .08 & & & & & & & & & & & & & & & & 7.7 \\
\hline .607 & & & & & & & 22 & & & & & & & & & .1 \\
\hline 173 & 0 & 4.05 & 2.67 & & & & 5 & & & & & & & & 39 & 0.6 \\
\hline .700 & & & & & & & & & & & & & & & & 3 \\
\hline 2.540 & & & & & & & & & & & & & & & & 7.6 \\
\hline 5.08 & & & & & & & 22 & & & & & & & & 32 & 8.2 \\
\hline 62 & & & & & & & & & & & & & & & 30 & .2 \\
\hline .14 & & & & & & & & & & & & & & & 17 & 0.4 \\
\hline 2.713 & & & & & & & & & & & & & & & & 9 \\
\hline 7.607 & .493 & & 3.93 & & & & 19833 & & & & & & 52 & & 7.08 & 8. \\
\hline 7.633 & 3.493 & 93.99 & 44.00 & 344.76 & 3494.8 & 5.35 & 19869 & 0.665 & 7629 & 42.26 & 45.53 & 1.10 & 2.53 & 11.27 & 7.10 & 3.6 \\
\hline
\end{tabular}


Channel Specimen

Experiment 5

Date: 8 August 1990

Time: 15:05:29

$\begin{array}{cccccccc}T A & T B & M & P O & P 0 . P 1 & V f & \text { Ot } & \text { Hqt } \\ K & K & k g / h & k P g & k P a & \% & W & x \\ 300.34 & 672.82 & 13.33 & 3525.0 & 26.79 & 75.33 & 7160.0 & 1.04\end{array}$

Hot-side Temperatures:

$\begin{array}{ccc}X & Y & T w \\ \mathrm{~cm} & \mathrm{~cm} & K \\ 1.588 & 0.655 & 512.20 \\ 2.540 & 0.655 & 540.21 \\ 5.080 & 0.655 & 605.45 \\ 7.620 & 0.655 & 675.81 \\ 10.160 & 0.655 & 734.67 \\ 12.700 & 0.655 & 756.64 \\ 13.653 & 0.655 & T 77.11\end{array}$

Insulated-Side Temperatures and Calculated Data:

\begin{tabular}{|c|c|c|c|c|c|c|c|c|c|c|c|c|c|c|c|c|}
\hline \multirow[b]{2}{*}{$x$} & \multirow[b]{2}{*}{ y } & \multirow[b]{2}{*}{$T w$} & \multirow[b]{2}{*}{ Tf } & \multirow[b]{2}{*}{ Taw } & \multirow[b]{2}{*}{$\mathbf{P}$} & \multirow[b]{2}{*}{$v$} & \multirow[b]{2}{*}{$\mathbf{E}$} & \multirow[b]{2}{*}{ PR } & \multirow{2}{*}{\multicolumn{2}{|c|}{ h NU }} & \multirow[b]{2}{*}{ NUm } & \\
\hline & & & & & & & & & & & & Itw & itf & Ire & Wh & Hnu \\
\hline $\mathrm{cm}$ & $\mathrm{cm}$ & $K$ & $K$ & $K$ & $\mathrm{kPa}$ & $\mathrm{m} / \mathrm{s}$ & & & $H /\left(m^{2} \cdot k\right)$ & & & $\%$ & $K$ & $\%$ & $x$ & $\%$ \\
\hline 0.000 & 0.965 & 79.29 & 00.23 & 00.33 & 525.0 & 33.68 & 873 & .665 & 5301 & 32.19 & 36.61 & & 2.03 & 1.27 & 1.64 & \\
\hline 1.270 & & 59.42 & 29.98 & 30.10 & 522.7 & 6.98 & 323 & .665 & 435 & 5.27 & 30.31 & 1.10 & 3.24 & 1.27 & 4.81 & 6.88 \\
\hline 2.527 & 0.330 & 03.36 & 59.67 & 59.81 & 520.5 & 0.28 & 850 & 0.665 & 618 & 19.44 & 23.39 & .10 & 4.28 & 1.27 & & 7.27 \\
\hline 3.810 & 0.318 & 36.95 & 87.96 & 88.12 & 518.3 & 3.43 & 454 & 0.666 & & 17.43 & 20.84 & & 5.70 & & & 7.68 \\
\hline 5.080 & 0.318 & 67.53 & 16.26 & 16.45 & 3516.1 & 46.58 & 102 & 0.666 & 3455 & 16.80 & 19.92 & & 7.28 & & & 8.17 \\
\hline 7.620 & 0.330 & 32.49 & 74.14 & 474.37 & 3511.6 & $53 \Omega 4$ & 6493 & 0.666 & & 14.82 & 17.37 & & 10.55 & & & 9.38 \\
\hline 8.903 & 0.305 & 66.52 & 03.36 & 503.63 & 3509.3 & 56.31 & 3230 & 0.666 & & 13.77 & 16.07 & & 1.85 & & & 9.82 \\
\hline 0.160 & 0.318 & 591.69 & 31.78 & 32.08 & & & 997 & 0.666 & & & & & & & & .61 \\
\hline & 0.318 & 09.75 & 59.79 & 560.13 & & & 5787 & 0.666 & & & & & & & & \\
\hline & & & 87.44 & & & & 596 & 0.666 & & 14.08 & 15.84 & & 16.02 & & & \\
\hline .970 & & & 17.01 & & & & 408 & 0.666 & & 16.31 & 18.10 & & 17.62 & & & 5.22 \\
\hline 15.240 & 0.953 & 27.34 & 45.88 & 646.32 & 3498.2 & 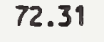 & 5238 & 0.666 & & 17.34 & 18.51 & & 19.09 & & & 27.29 \\
\hline 5.080 & 2.235 & 93.93 & 24.26 & 424.43 & & 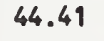 & 559 & 0.666 & & 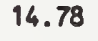 & 17.78 & & 7.28 & & & 7.87 \\
\hline 7.607 & 2.05 & 55.02 & 85.80 & 486.02 & & - & 974 & 0.666 & & 13.64 & 16.08 & & 10.54 & & & 9.07 \\
\hline 10.173 & -2.223 & 16.34 & 48.04 & 548.32 & & & 95 & 0.666 & & 12.39 & 14.36 & & 13.21 & & & 0.30 \\
\hline 12.700 & -2.223 & 747.44 & 00 & 07.56 & & & 117 & 0.666 & & 13.72 & 15.38 & & 16.02 & & & 13.29 \\
\hline 2.540 & 2.197 & 16.70 & 365.52 & 365.64 & 3520.5 & 37.46 & 7105 & 0.665 & & 18.26 & 22.09 & & 4.29 & & & 7.21 \\
\hline 5.080 & 2.223 & 585.99 & 427.05 & 427.21 & & & 6387 & 0.666 & & 15.71 & 18.70 & & 7.28 & & & 8.03 \\
\hline 7.620 & 2.223 & 663.26 & 490.29 & 490.50 & 3511.6 & 50.18 & 5806 & 0.666 & & 13.25 & 15.65 & & 10.55 & & .52 & 8.98 \\
\hline 10.147 & 2.223 & 716.84 & 552.97 & 553.24 & & & 5342 & 0.666 & & 12.66 & 14.60 & & 13.18 & & .22 & 10.45 \\
\hline 12.713 & 2.223 & 747.15 & 614.43 & & & 62.90 & 4964 & 0.666 & & 14.39 & 16.02 & & 16.03 & & & 13.87 \\
\hline 7.607 & -3.493 & 670.50 & 493.91 & 494.12 & & & 5660 & 0.666 & & 12.92 & 15.29 & & 10.54 & & & 8.89 \\
\hline 7.633 & 3.493 & 701.50 & 510.56 & 510.75 & 3511.6 & 47.28 & 5111 & 0.666 & 2762 & 11.67 & 13.90 & 1.71 & 10.57 & 11.27 & 7.06 & 8.61 \\
\hline
\end{tabular}


Table 3 (continued)

Channel specimen

Experiment 5

Date: 8 August 1990

Time: $15: 13: 22$

\begin{tabular}{|c|c|c|c|c|c|c|c|}
\hline TA & TB & M & PO & P0-P1 & $v f$ & $Q t$ & Wat \\
\hline$K$ & $\mathbf{K}$ & $\mathrm{kg} / \mathrm{h}$ & $\mathrm{KPB}$ & $\mathrm{KPa}$ & $x$ & $H$ & $x$ \\
\hline 0.08 & 592.34 & 17.31 & 3535.4 & 38.03 & 75.43 & 7291.0 & .05 \\
\hline
\end{tabular}

Hot-side Temperatures:

$\begin{array}{ccc}X & Y & T H \\ \mathrm{~cm} & \mathrm{~cm} & K \\ 1.588 & 0.655 & 473.94 \\ 2.540 & 0.655 & 493.94 \\ 5.080 & 0.655 & 542.82 \\ 7.620 & 0.655 & 595.95 \\ 10.160 & 0.655 & 633.09 \\ 12.700 & 0.655 & 664.45 \\ 13.653 & 0.655 & 682.45\end{array}$

Insulated-Side Temperatures and Calculated Data:

\begin{tabular}{|c|c|c|c|c|c|c|c|c|c|c|c|c|c|c|c|c|}
\hline$x$ & $Y$ & $H$ & $f$ & 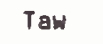 & $\mathbf{P}$ & v & RE & $\mathbf{R}$ & h & NU & "I & $t w$ & tf & re & Wh & Inu \\
\hline $\mathrm{cm}$ & $\mathrm{cr}$ & $K$ & K & $k$ & $k$ & $\mathrm{~m} / \mathrm{s}$ & & & $\cos$ & & & $K$ & K & $x$ & $x$ & $\%$ \\
\hline 0.000 & .965 & 50.80 & $\$ 9.90$ & 6 & 3535.4 & 5 & 1580 & 665 & 19 & 42.64 & 7.20 & 0 & 1.62 & 7 & 2 & .7 \\
\hline 1.2 & 8 & 3 & 2 & 0 & $32 ?$ & 7.12 & 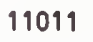 & 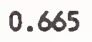 & 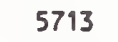 & 801 & i & 0 & 6 & 7 & 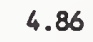 & 6.9 \\
\hline 2.52 & .330 & 8.30 & 6.29 & 66.50 & 20 & .49 & S & 5 & 4731 & 60 & 4 & 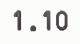 & 7 & & 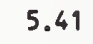 & 7.3 \\
\hline 3.81 & .318 & 3.60 & .37 & 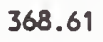 & & 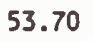 & 72 & & & & & & 8 & & & 7.7 \\
\hline 5.080 & 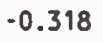 & 4 & 5 & 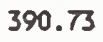 & & .92 & 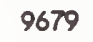 & & & & & & 1 & & & 2 \\
\hline 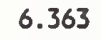 & & 2 & & & & & 9310 & & & & & & 7.00 & & & 8.9 \\
\hline 7.62 & & 0 & 1 & 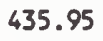 & & & 8 & & & & & & 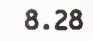 & & & 6 \\
\hline .90 & 5 & 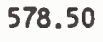 & 2 & 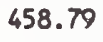 & & $0 . \infty 0$ & 68 & & & & & & 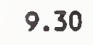 & & & 2 \\
\hline 0 & 8 & 3 & 0.60 & 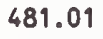 & & 0.14 & 889 & & & & & & 5 & & & 2 \\
\hline .430 & 18 & 5 & 02.45 & 1 & & 36 & 35 & & & & & & + & & & 5 \\
\hline 2.700 & 0.305 & 629.81 & 24.03 & 24. & & 6.54 & 201 & 66 & & & 6 & & 7 & & & .7 \\
\hline 3.970 & 0.318 & 647.75 & 47.09 & 47.63 & 0.5 & .95 & 7669 & .666 & & & 8 & & 3 & & & .2 \\
\hline 5.240 & 0.953 & 632.35 & 69.62 & 70.21 & 7.4 & 3.28 & 7456 & .666 & & & 6.62 & & 14.98 & & & 27.7 \\
\hline 2.527 & 2.223 & 471.27 & 49.65 & 49.85 & 9.1 & 7.55 & 9736 & 0.665 & 4356 & & 8.12 & & 3.37 & & & 7.2 \\
\hline 5.080 & 2.235 & 529.00 & 97.01 & 97.25 & 2.7 & 3.99 & 8927 & 0.666 & 4034 & 20.27 & 3.74 & & 5.71 & $?$ & & 7.9 \\
\hline 7.607 & -2.235 & 572.69 & 45.18 & 45.48 & .4 & 0.55 & 8251 & .666 & 4218 & 59 & 2.50 & 9 & 8.27 & 7 & 4 & 9.2 \\
\hline .173 & -2.223 & 616.32 & 93.89 & 94.27 & .0 & .22 & 7680 & .666 & 0 & 68 & 1.10 & 7 & 10.36 & 7 & 1 & 10.8 \\
\hline 12.700 & -2.223 & 650.78 & 40.21 & 540.67 & 7 & .59 & 7217 & 66 & 4740 & 26 & 1.34 & 1.51 & 12.57 & 7 & 12 & 13.2 \\
\hline 2.540 & 2.197 & 470.00 & 71 & 0 & 1 & .93 & 9561 & .665 & 7 & 26 & 8.50 & 1.10 & 3.38 & 11 & 5.30 & 7.2 \\
\hline 5.080 & 2.223 & 523.02 & 98.58 & 398.82 & 3522.7 & 3.33 & 8761 & 66 & 4280 & 6 & 0 & 1.10 & 5.71 & 7 & 8 & 8.0 \\
\hline 7.620 & 2.223 & 577.33 & 47.79 & 448.09 & & & 9097 & & 4152 & 19.21 & 9 & $?$ & 8 & $?$ & 7 & 2 \\
\hline & 2.223 & & & & & & & & 4539 & 19.55 & ; & 1.36 & 4 & 7 & 8 & 11.1 \\
\hline 797 & .223 & 650.3 & 44.36 & & & 2 & 70 & 6 & & & 7 & & 8 & 7 & 2.80 & 13.7 \\
\hline 7.607 & -3.493 & 592.41 & 455.72 & 456.00 & & & & 6 & & & 0.77 & & 7 & 7 & 8 & 8.9 \\
\hline 7.633 & 3.493 & 610.14 & 465.52 & 465.78 & 3516.4 & 55.73 & 7045 & 0.666 & 3717 & 16.74 & 19.43 & 1.35 & 8.29 & 11.27 & 7.23 & 0. \\
\hline
\end{tabular}




\section{Table 3 (continued)}

Chamel specimen

Experiment 5

Date: 8 August 1990

Time: 15:20:16

$\begin{array}{cccccccc}\text { TA } & T B & M & P O & P 0-P 1 & V f & \text { Ot } & \text { Wqt } \\ K & K & k g / h & K P a & K P a & X & W & X \\ 299.87 & 513.32 & 23.81 & 3547.9 & 59.13 & 73.48 & 7326.0 & 1.07\end{array}$

Hot-side Temperatures:

$\begin{array}{ccc}X & Y & T W \\ \mathrm{~cm} & \mathrm{Cm} & K \\ 1.588 & 0.655 & 438.77 \\ 2.540 & 0.655 & 452.11 \\ 5.080 & 0.655 & 487.20 \\ 7.620 & 0.655 & 525.03 \\ 10.160 & 0.655 & 552.10 \\ 12.700 & 0.655 & 576.65 \\ 13.653 & 0.655 & 591.26\end{array}$

Insulated-Side Temperatures and Calculated Data:

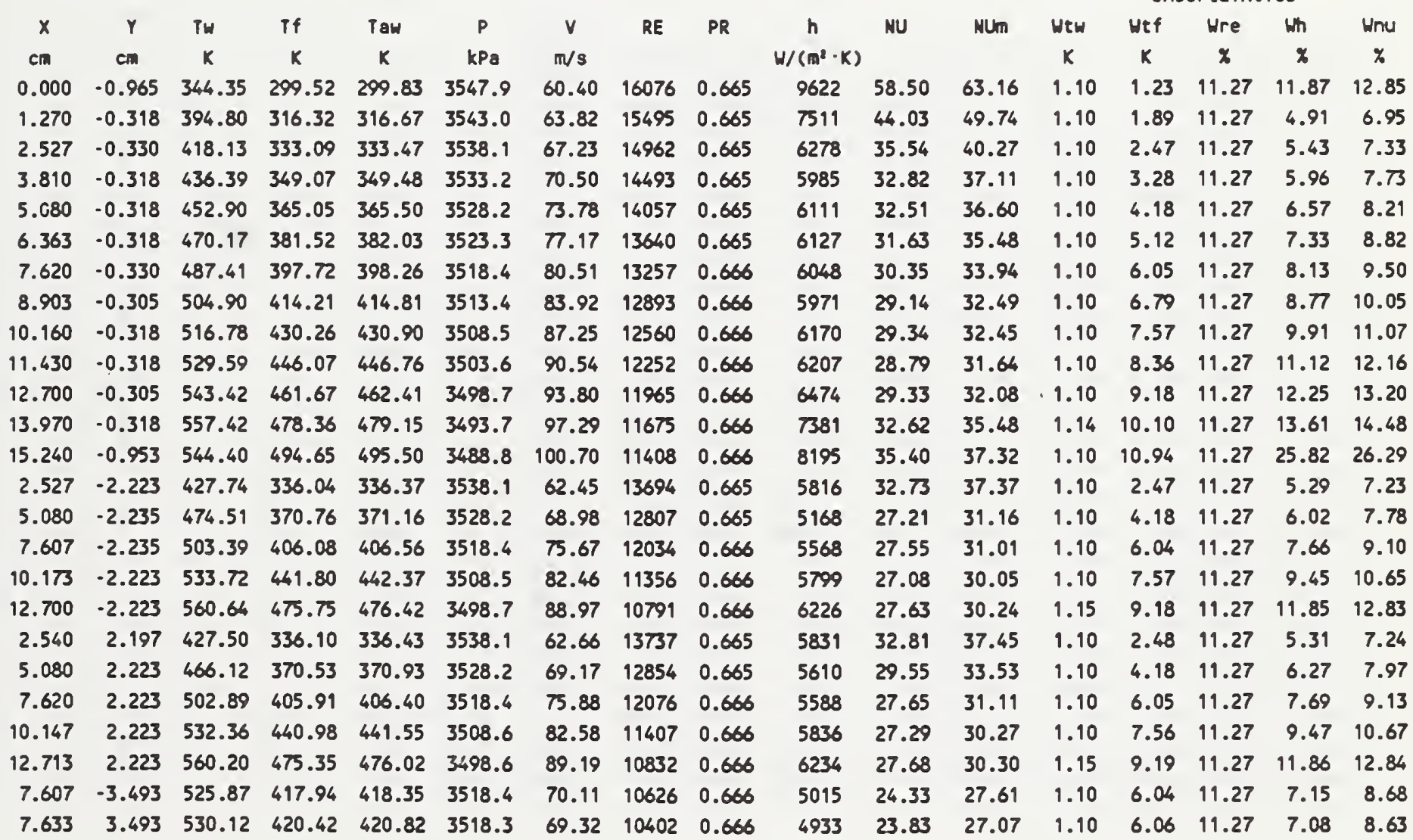


Channel Specimen

Experiment 5

Date: 8 August 1990

Time: 15:26:11

$\begin{array}{cccccccc}T A & T B & M & P O & P 0-P 1 & V f & \text { Ot } & \text { Wat } \\ K & K & \mathrm{~kg} / \mathrm{h} & \mathrm{KPa} & \mathrm{kPa} & \chi & W & \chi \\ 299.66 & 463.95 & 31.08 & 3554.0 & 87.01 & 75.49 & 7358.0 & 1.11\end{array}$

Hot-side Temperatures:

$\begin{array}{ccc}X & Y & T H \\ \mathrm{~cm} & \mathrm{~cm} & K \\ 1.588 & 0.655 & 416.43 \\ 2.540 & 0.655 & 425.88 \\ 5.080 & 0.655 & 452.84 \\ 7.620 & 0.655 & 481.88 \\ 10.160 & 0.655 & 502.86 \\ 12.700 & 0.655 & 521.93 \\ 13.653 & 0.655 & 533.78\end{array}$

Insulated-Side Temperatures and Calculated Data:

\begin{tabular}{|c|c|c|c|c|c|c|c|c|c|c|c|c|c|c|c|c|}
\hline$x$ & $Y$ & $T_{H}$ & Tf & Тан & $P$ & $v$ & RE & $\mathbf{R}$ & h & U & m & Wtw & Wtf & Wre & Wh & Wnu \\
\hline $\mathrm{cm}$ & $\mathrm{cm}$ & K & K & K & $\mathrm{kPa}$ & m & & & $W /\left(m^{2} \cdot k\right)$ & & & $k$ & K & $x$ & $x$ & $x$ \\
\hline .000 & .965 & 34.05 & $\$ 9.06$ & 99.59 & 54.0 & 4 & 205 & .665 & 12483 & 75.97 & 0.74 & 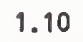 & 00 & 27 & 2.07 & 107 \\
\hline .270 & .318 & 6 & 1.84 & 2 & 6.8 & 3 & 0615 & .665 & 379 & 1 & 6 & 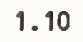 & 49 & & .00 & .01 \\
\hline 2.527 & 0.330 & 22.95 & 24.60 & 25.23 & 6 & 3 & 20064 & 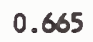 & 881 & 0 & 3 & 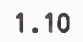 & 92 & 7 & .48 & 7.36 \\
\hline .810 & -0.318 & 13 & 36.76 & 37.43 & 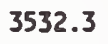 & 70 & 2569 & 政 & 695 & 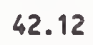 & 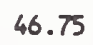 & 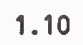 & 54 & 7 & 5.96 & 7.73 \\
\hline .080 & -0.318 & 19.67 & 48.91 & 49.64 & 5.0 & 07 & 19103 & .665 & 7660 & 42.03 & 6.52 & 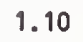 & .22 & 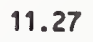 & 6.52 & 8.17 \\
\hline .363 & 0.318 & 32.91 & 61.45 & 52.23 & 7.7 & 56 & 18649 & .665 & 574 & 8 & 40 & 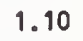 & .95 & 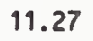 & 7.21 & 8.73 \\
\hline .620 & -0.330 & 46.18 & $73 . \pi$ & 74.61 & 0.5 & 10 & 18228 & .665 & 566 & 1 & 6 & 1.10 & .66 & 7 & 7.96 & 9.36 \\
\hline .903 & -0.305 & 60.18 & 86.31 & 87.21 & 3.2 & 3 & 17821 & .666 & $7 ٪ 05$ & 1 & 4 & 1.10 & .23 & 7 & 3.50 & 9.82 \\
\hline .160 & -0.318 & 05 & 98.52 & 8 & 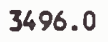 & 7 & 7445 & .666 & 7650 & 4 & & 1.10 & 3 & 7 & .58 & 0.77 \\
\hline .430 & -0.318 & 17 & 10.54 & 411.57 & 0 & 8 & 17093 & .666 & 7639 & 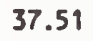 & 4 & 1.10 & .44 & 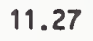 & .65 & 1.73 \\
\hline .700 & -0.305 & 489.92 & 22.41 & 423.50 & 3481.5 & 6 & 16762 & .666 & 93 & 8. & 7 & 1.0 & 7.07 & 7 & .66 & 2.65 \\
\hline .970 & -0.318 & 01.57 & 35.10 & 36.26 & 74.3 & .38 & 16424 & .666 & 888 & 6 & S.2. & & 7.78 & 7 & .71 & 3.63 \\
\hline .240 & -0. & 489 & 47.48 & 1 & 0 & 3 & 16110 & 0 & קנדי & 45.17 & 47.48 & & 3 & & 20 & 24.70 \\
\hline 2.527 & -2.2 & 7 & 27.20 & 3 & & & 18187 & 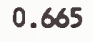 & & & & & 2 & & 5 & 7.27 \\
\hline 5.080 & . & 1 & 20 & & & & 17 & & & & & & & & & 7.64 \\
\hline 7.607 & & & & & & & & & & & & & & & & 8.94 \\
\hline 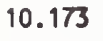 & & & & & & & & & & & & & & & & 0.42 \\
\hline .700 & -2.223 & & & & 3 & & & & & 20 & 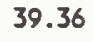 & & 7 & & 11.35 & 12.37 \\
\hline 2.540 & 2.197 & 4 & 327.02 & & 39.5 & & 1 & & & & & & 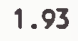 & & 5. & 7.26 \\
\hline 5.080 & 2.223 & 8 & 0.68 & 353 & 25.0 & & 11 & & 7 & 38.12 & 42 & 1.0 & 6 & & 2 & 7.93 \\
\hline 7.620 & 2.223 & 458 & 380.28 & 381 & & & & & 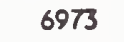 & 36.08 & & & 4.66 & & 7.52 & 8.99 \\
\hline .147 & 2.223 & 481 & 407.03 & 407 & .1 & .64 & 15843 & 0.666 & حل & 35.49 & 38. & 10 & 5.82 & & 9.11 & 10.35 \\
\hline 12.713 & 2.223 & 503.74 & 433.25 & 434.22 & 3481.5 & 107.48 & 15178 & 0.666 & 7582 & 35.88 & 38.98 & 1.10 & 7.08 & 11.27 & 11.22 & 12.25 \\
\hline 7.607 & -3.493 & 483.63 & 393.14 & 393.72 & 3510.6 & 83.66 & 14015 & 0.666 & 6023 & 30.47 & 34.15 & 1.10 & 4.66 & 11.27 & 6.84 & 8.43 \\
\hline 7.633 & 3.493 & 482.50 & 392.74 & 393.34 & 3510.5 & 84.21 & 14130 & 0.666 & 6073 & 30.74 & 34.42 & 1.10 & 4.67 & 11.27 & 6.89 & 8.47 \\
\hline
\end{tabular}


Table 3 (continued)

Channel Specimen

Experiment 5

Date: 8 August 1990

Time: 15:32:04

$\begin{array}{cccccccc}T A & T B & M & P O & P O-P 1 & V f & \text { ot } & \text { Hqt } \\ K & K & \mathrm{~kg} / \mathrm{h} & \mathrm{KPa} & \mathrm{KPa} & \boldsymbol{Z} & \mathrm{H} & \%\end{array}$

$\begin{array}{llllllll}299.19 & 424.49 & 40.64 & 3561.3 & 131.64 & 75.55 & 7335.0 & 1.17\end{array}$

Hot-side Temperatures:

$\begin{array}{ccc}X & Y & T w \\ c m & c m & K \\ 1.588 & 0.655 & 397.64 \\ 2.540 & 0.655 & 404.16 \\ 5.080 & 0.655 & 424.73 \\ 7.620 & 0.655 & 446.98 \\ 10.160 & 0.655 & 462.97 \\ 12.700 & 0.655 & 477.46 \\ 13.653 & 0.655 & 487.30\end{array}$

Insulated-side Temperatures and Calculated Data:

\begin{tabular}{|c|c|c|c|c|c|c|c|c|c|c|c|c|c|c|c|c|}
\hline $\begin{array}{r}x \\
c m\end{array}$ & $\begin{array}{c}Y \\
\mathrm{~cm}\end{array}$ & $\begin{array}{l}T w \\
K\end{array}$ & $\begin{array}{r}\text { if } \\
k\end{array}$ & $\begin{array}{c}\text { Taw } \\
K\end{array}$ & $\begin{array}{c}\mathrm{P} \\
\mathrm{KPa}\end{array}$ & $\begin{array}{c}v \\
\mathrm{~m} / \mathrm{s}\end{array}$ & RE & PR & $\stackrel{h}{h /\left(m^{2} \cdot K\right)}$ & MU & 的 & $\begin{array}{l}\text { WEW } \\
K\end{array}$ & $\begin{array}{l}\text { wtf } \\
k\end{array}$ & $\begin{array}{l}\text { Wre } \\
x\end{array}$ & $\begin{array}{l}\text { Wh } \\
x\end{array}$ & $\begin{array}{l}\text { Hnu } \\
\%\end{array}$ \\
\hline 0.000 & .965 & 325.62 & 298.15 & 299.07 & 3561.3 & 104.26 & 28062 & 0.665 & 16154 & 98.50 & 103.39 & 1.10 & 0.83 & 11.27 & 12.42 & 13.36 \\
\hline 1.270 & .318 & 59.04 & 307.77 & 308.75 & 3550.4 & 07.89 & 27470 & 0.665 & 11685 & 69.77 & 75.94 & .10 & 1.17 & 11.27 & 5.17 & 7.13 \\
\hline 2.527 & .330 & 72.40 & 17.37 & 318.42 & 3539.5 & 1.53 & 26906 & 0.665 & 9856 & 57.65 & 62.95 & 1.10 & 1.49 & 11.27 & 5.59 & 7.45 \\
\hline 3.810 & .318 & 83.37 & 326.52 & 327.63 & 528.4 & 15.04 & 26394 & 0.665 & 9342 & 53.60 & 58.55 & 1.10 & 1.95 & 11.27 & 6.00 & 7.76 \\
\hline 5.080 & 0.318 & 22.82 & 335.66 & 336.84 & 3517.4 & 8.58 & 25904 & 0.665 & 53 & 53.80 & 58.66 & 10 & 2.47 & .27 & 6.50 & 8.16 \\
\hline 6.363 & -0.318 & 02.83 & 345.09 & 346.35 & 3506.4 & 122.24 & 25421 & 0.665 & 9573 & 52.92 & 57.62 & 1.10 & 3.02 & 11.27 & 7.14 & 8.67 \\
\hline 7.620 & 0.330 & 412.98 & 354.35 & 355.69 & 3495.5 & 125.85 & 24967 & 0.665 & 9423 & 51.16 & 55.65 & 1.10 & 3.56 & 11.27 & 7.81 & 9.23 \\
\hline 8.903 & .305 & 424.22 & 363.79 & 365.20 & 3484.4 & 129.56 & 24524 & 0.665 & 9127 & 48.68 & 52.97 & 1.10 & 4.00 & 11.27 & 8.25 & 9.60 \\
\hline .160 & 0.318 & 430.53 & 372.96 & 374.46 & 3473.6 & 133.19 & 24110 & 0.666 & 9461 & 49.61 & 53.69 & 1.10 & 4.45 & 11.27 & 9.27 & 10.50 \\
\hline 1.430 & -0.318 & 438.47 & 382.00 & 383.58 & 3462.6 & 136.80 & 23719 & 0.666 & 9378 & 48.38 & 52.19 & 1.10 & 4.91 & 11.27 & 10.22 & 11.34 \\
\hline 2.700 & .305 & 446.66 & 390.92 & 392.58 & 3451.6 & 140.38 & 23347 & 0.666 & 9709 & 49.31 & 53.06 & 1.10 & 5.40 & 11.27 & 1.12 & 12.16 \\
\hline .970 & 0.318 & 56.38 & 400.46 & 402.21 & 440.7 & 44.22 & 22965 & 0.666 & $106 \pi 7$ & 53.34 & .32 & 1.10 & 5.94 & 11.27 & 11.88 & 12.86 \\
\hline 15.240 & -0.953 & 6.19 & 409.77 & 411.61 & 3429.7 & 147.99 & 22606 & 0.666 & 11602 & 57.05 & .79 & 1.10 & 6.43 & 11.27 & 22.74 & 23.27 \\
\hline 2.527 & -2.223 & 378.07 & 319.69 & 320.55 & 3539.5 & 101.29 & 24142 & 0.665 & 9251 & 53.84 & 59.04 & 1.10 & 1.49 & 11.27 & 5.46 & 7.35 \\
\hline 5.080 & -2.235 & 414.02 & 340.01 & 340.99 & 3517.4 & 108.28 & 23154 & 0.665 & 7322 & 40.88 & 45.56 & 0 & 2.47 & 11.27 & 5.72 & 7.54 \\
\hline 7.607 & -2.235 & 425.29 & 360.66 & 361.79 & 3495.6 & 115.46 & 22243 & 0.665 & 8501 & 45.61 & 14 & 1.10 & 3.56 & 11.27 & 7.28 & 8.79 \\
\hline .173 & -2.223 & 441.40 & 381.55 & 382.82 & 3473.5 & 122.82 & 21405 & 0.666 & 9053 & 46.74 & 50.64 & 1.10 & 4.45 & 11.27 & 8.98 & 10.24 \\
\hline 12.700 & -2.223 & 457.96 & 401.40 & 402.82 & 3451.6 & 129.93 & 20674 & 0.666 & 9521 & 47.48 & 51.05 & 1.10 & 5.40 & 11.27 & 10.94 & 12.00 \\
\hline 2.540 & 2.197 & 378.80 & 319.36 & 320.26 & 3539.4 & 103.09 & 24613 & 0.665 & 9083 & 52.90 & 58.11 & 1.10 & 1.50 & 11.27 & 5.43 & 7.33 \\
\hline 5.080 & 2.223 & 401.64 & 339.19 & 340.21 & 3517.4 & 110.06 & 23628 & 0.665 & 8705 & 48.68 & 53.42 & 1.10 & 2.47 & 11.27 & 6.19 & 7.91 \\
\hline 7.620 & 2.223 & 423.05 & 359.57 & 360.73 & 3495.5 & 117.29 & 22708 & 0.665 & 8662 & 46.56 & 50.92 & 1.10 & 3.56 & 11.27 & 7.38 & 8.87 \\
\hline 10.147 & 2.223 & 441.08 & 379.75 & 381.06 & 3473.7 & 124.54 & 21878 & 0.666 & 8841 & 45.80 & 49.73 & 1.10 & 4.44 & 11.27 & 8.80 & 10.08 \\
\hline 12.713 & 2.223 & 457.79 & 399.54 & 401.00 & 3451.5 & 131.77 & 21130 & 0.666 & 9252 & 46.29 & 49.89 & 1.10 & 5.40 & 11.27 & 10.68 & 11.76 \\
\hline 7.607 & -3.493 & 448.18 & 372.34 & 373.20 & 3495.6 & 100.85 & 18423 & 0.665 & 7200 & 37.80 & 41.86 & 1.10 & 3.56 & 11.27 & 6.58 & 8.22 \\
\hline 7.633 & 3.493 & 443.77 & 370.25 & 371.15 & 3495.4 & 103.50 & 19083 & 0.665 & 7434 & 39.18 & 43.28 & 1.10 & 3.57 & 11.27 & 6.71 & 8.32 \\
\hline
\end{tabular}




\section{Table 3 (continued)}

Channel Specimen

Experiment 6

Date: 10 August 1990

Time: 14:04:43

$\begin{array}{cccccccc}T A & T B & H & P O & P O-P 1 & V f & \text { ot } & \text { Hat } \\ K & K & \mathrm{~kg} / \mathrm{h} & \mathrm{KPa} & \mathrm{KPa} & \boldsymbol{K} & \mathrm{H} & \% \\ 300.87 & 709.80 & 3.78 & 6942.2 & 1.88 & 25.24 & 2226.0 & 1.03\end{array}$

Hot-side Temperatures:

$\begin{array}{ccc}X & Y & T W \\ c m & c m & K \\ 1.588 & 0.655 & 486.16 \\ 2.540 & 0.655 & 521.26 \\ 5.080 & 0.655 & 601.04 \\ 7.620 & 0.655 & 671.32 \\ 10.160 & 0.655 & 729.72 \\ 12.700 & 0.655 & 772.87 \\ 13.653 & 0.655 & 782.61\end{array}$

Insulated-Side Temperatures and Calculated Data:

\begin{tabular}{|c|c|c|c|c|c|c|c|c|c|c|c|c|c|c|c|c|}
\hline $\begin{array}{c}x \\
c m\end{array}$ & $\begin{array}{c}Y \\
\mathrm{~cm}\end{array}$ & $\begin{array}{r}T H \\
K\end{array}$ & If & $\begin{array}{c}\text { Taw } \\
\text { K }\end{array}$ & $\stackrel{\mathrm{P}}{\mathrm{KPa}}$ & $\begin{array}{c}\mathrm{V} \\
\mathrm{m} / \mathrm{s}\end{array}$ & RE & R & $w /\left(m^{2} \cdot k\right)$ & NU & Um & $\begin{array}{l}\text { Ntw } \\
\text { K }\end{array}$ & $\begin{array}{c}\text { Htf } \\
\text { K }\end{array}$ & $\begin{array}{c}\text { Wre } \\
*\end{array}$ & $\begin{array}{l}\text { th } \\
\%\end{array}$ & $\begin{array}{l}\text { Wnu } \\
\%\end{array}$ \\
\hline 0.000 & 0.965 & 90.13 & 0.8 & 00.87 & 442.2 & 4.73 & 2394 & Sc & 1458 & 8. & 10.08 & 1.10 & 2.21 & 1.27 & 1.60 & 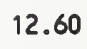 \\
\hline 270 & 318 & 7.45 & 4.94 & & & 24 & 8 & 662 & & & & & 55 & .27 & .04 & \\
\hline .527 & & 6.94 & .93 & & & & 87 & 663 & & & & & 69 & .27 & .60 & 2.8 \\
\hline 3.810 & .318 & 9.63 & 01.34 & 1.34 & 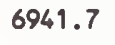 & 5.25 & 972 & 0.663 & 666 & 5.28 & 6.28 & 11 & 6.26 & 1.27 & .16 & 7.8 \\
\hline .080 & .318 & 37.29 & 33.75 & - & & 74 & 870 & .664 & & & & & 7.99 & .27 & & \\
\hline .363 & .318 & 23.88 & 57.18 & 37.19 & .4 & .25 & 777 & .664 & 1 & .68 & & 0 & 9.79 & 11.27 & .63 & \\
\hline .620 & .330 & 7.24 & 0.04 & 0.04 & & 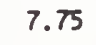 & 696 & .664 & & & & 34 & .59 & .27 & .58 & \\
\hline .903 & .305 & 8.29 & 3.51 & 32 & & 26 & 522 & 0.664 & & & & 66 & .01 & .27 & .50 & 0. \\
\hline .160 & .318 & 6.85 & 66.08 & 566.08 & & .75 & 1557 & 0.665 & 68 & 4. & 4.76 & 77 & 14.48 & 11.27 & 0.61 & . \\
\hline .430 & .318 & 40.95 & 98.17 & 598 & & .24 & 1498 & 65 & & & & & 16.00 & 27 & 14 & د. \\
\hline .700 & 305 & 1.79 & 29.85 & 29.86 & & .72 & 446 & 0.665 & 1 & o. & & 6 & 17.58 & 27 & .12 & 4. \\
\hline .970 & .318 & 72.04 & 63.72 & 63.7 & .5 & .24 & 394 & 0.665 & 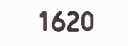 & 5. & & 2.00 & 19.35 & .27 & .41 & 9. \\
\hline .240 & 0.953 & 30.84 & 96.81 & 96.82 & 3 & .74 & 347 & 0.665 & & 6.45 & 7 & 95 & 20.95 & .27 & 5.23 & 5. \\
\hline 2.527 & 2.223 & 4.23 & 70.81 & & & 63 & 2025 & 63 & & & & & 4.69 & 27 & 52 & 7 \\
\hline 5.080 & 2.235 & 91.54 & 37. & & & .62 & 1809 & 34 & & & & & 7.99 & 27 & 30 & 1.0 \\
\hline 7.607 & -2.235 & 666.71 & 05.17 & & & 62 & 1639 & 0.664 & & 4. & & 7 & 11.57 & .27 & .40 & . \\
\hline .173 & 2.223 & 730.84 & 73.71 & & & .63 & 1501 & 65 & & 18 & & & 14.50 & .27 & .27 & 1. \\
\hline .700 & 2.223 & 3.44 & & & & & 1393 & & & & & 2.01 & 17.58 & 9.27 & 3.70 & 4. \\
\hline 2.540 & 2.197 & 515.65 & 71.96 & & & 5.59 & 1997 & 0.663 & & 5. & 7.00 & 1. & 4.71 & .27 & 5.52 & 7 \\
\hline 5.080 & 2.223 & 8.6 & 38. & & & & 1785 & & & & & & 7.99 & 27 & 36 & . \\
\hline 7.620 & 2.223 & 671.2 & $0 ? .87$ & & & & 1615 & 0 & & & & & 11.59 & .27 & .34 & 9. \\
\hline .147 & 2.223 & 729.88 & 76.16 & & & & 1480 & & & & & & 14.47 & 1.27 & 0.44 & 1.5 \\
\hline 12.713 & 2.223 & 771.80 & 643.13 & 643.14 & & 9.55 & 1371 & 0.665 & 1239 & 4.45 & 4.92 & 2.00 & 17.60 & 1.27 & 14.46 & 15.2 \\
\hline 7.607 & -3.493 & 674.40 & 509.45 & 509.46 & 6941.3 & 7.53 & 1596 & 0.664 & $\infty$ & 4.18 & 4.88 & 1.61 & 11.57 & 1.27 & 8.27 & \\
\hline 7.633 & 3.493 & 688.21 & 517.53 & 517.54 & 6941.3 & 7.38 & 1525 & 0.664 & 960 & 4.00 & 4.68 & 1.66 & 11.60 & 19.27 & 8.09 & \\
\hline
\end{tabular}


Channel Specimen

Experiment 6

Date: 10 August 1990

Time: $14: 16: 47$

$\begin{array}{cccccccc}\text { TA } & \text { TB } & M & P O & P 0-P 1 & V F & \text { Ot } & \text { Wat } \\ K & K & \mathrm{~kg} / \mathrm{h} & \mathrm{KPa} & \mathrm{KPa} & \% & \mathrm{H} & \%\end{array}$

Hot-side Temperatures:

$\begin{array}{ccc}X & Y & T H \\ \mathrm{~cm} & \mathrm{Cm} & K \\ 1.588 & 0.655 & 419.18 \\ 2.540 & 0.655 & 438.39 \\ 5.080 & 0.655 & 483.43 \\ 7.620 & 0.655 & 527.77 \\ 10.160 & 0.655 & 570.34 \\ 12.700 & 0.655 & 599.82 \\ 13.653 & 0.655 & 604.14\end{array}$

Insulated-Side Temperatures and Calculated Data:

\begin{tabular}{|c|c|c|c|c|c|c|c|c|c|c|c|c|c|c|c|c|}
\hline$x$ & $Y$ & TH & Tf & Taw & $P$ & $v$ & RE & PR & $h$ & NU & NUm & Wtw & Wtf & Ure & Wh & Hnu \\
\hline $\mathrm{cm}$ & $\mathrm{cm}$ & $k$ & $k$ & $k$ & $\mathrm{kPa}$ & $\mathbb{N} / \mathrm{s}$ & & & $H /\left(m^{2} \cdot k\right)$ & & & $k$ & $k$ & $x$ & $x$ & $\%$ \\
\hline 0.000 & -0.965 & 352.77 & 299.11 & 299.11 & 6944.5 & 9.00 & 4602 & 0.661 & 2651 & 15.96 & 17.48 & 1.10 & 1.35 & 11.27 & 11.73 & 12.72 \\
\hline 1.270 & -0.318 & 397.34 & 318.55 & 318.55 & 6944.2 & 9.56 & 4413 & 0.662 & 2473 & 14.29 & 16.14 & 1.10 & 2.10 & 11.27 & 5.03 & 7.04 \\
\hline 2.527 & -0.330 & 425.83 & 337.95 & 337.95 & 6943.9 & 10.13 & 4241 & 0.662 & 2008 & 11.16 & 12.67 & 1.10 & 2.76 & 11.27 & 5.53 & 7.40 \\
\hline 3.810 & -0.318 & 450.08 & 356.44 & 356.45 & 6943.6 & 10.67 & 4092 & 0.663 & 1844 & 9.89 & 11.24 & 1.10 & 3.66 & 11.27 & 6.02 & 7.78 \\
\hline 5.080 & -0.318 & 471.89 & 374.93 & 374.94 & 6943.3 & 11.20 & 3954 & 0.663 & 1829 & 9.48 & 10.76 & 1.10 & 4.66 & 11.27 & 6.57 & 8.21 \\
\hline 6.363 & -0.318 & 493.82 & 394.01 & 394.02 & 6943.0 & 11.76 & 3824 & 0.663 & 1797 & 9.01 & 10.20 & 1.10 & 5.71 & 11.27 & 7.24 & 8.75 \\
\hline 7.620 & -0.330 & 515.12 & 412.76 & 412.77 & 6942.8 & 12.31 & 3704 & 0.664 & 1749 & 8.50 & 9.60 & 1.10 & 6.76 & 11.27 & 7.95 & 9.35 \\
\hline 8.903 & -0.305 & 536.99 & 431.86 & 431.88 & 6942.5 & 12.86 & 3592 & 0.664 & 1699 & 8.01 & 9.03 & 1.10 & 7.58 & 11.27 & 8.46 & 9.79 \\
\hline 10.160 & -0.318 & 555.95 & 450.44 & 450.46 & 6942.2 & 13.40 & 3490 & 0.664 & 1668 & 7.64 & 8.58 & 1.13 & 8.44 & 11.27 & 9.18 & 10.41 \\
\hline 11.430 & -0.318 & 572.49 & 468.76 & 468.77 & 6941.9 & 13.93 & 3396 & 0.664 & 1646 & 7.34 & 8.19 & 1.20 & 9.33 & 11.27 & 10.11 & 11.25 \\
\hline 12.700 & -0.305 & 585.96 & 486.83 & 486.85 & 6941.6 & 14.46 & 3309 & 0.664 & 1757 & 7.63 & 8.45 & 1.25 & 10.25 & 11.27 & 11.32 & 12.35 \\
\hline 13.970 & -0.318 & 591.16 & 506.17 & 506.18 & 6941.3 & 15.02 & 3221 & 0.664 & 2257 & 9.55 & 10.40 & 1.27 & 11.28 & 11.27 & 13.97 & 14.81 \\
\hline 15.240 & -0.953 & 574.90 & 525.04 & 525.07 & 6941.0 & 15.57 & 3141 & 0.664 & 2670 & 11.02 & 11.58 & 1.21 & 12.22 & 11.27 & 27.71 & 28.14 \\
\hline 5.080 & -2.235 & 483.26 & 379.29 & 379.30 & 6943.3 & 10.72 & 3710 & 0.663 & 1706 & 8.78 & 10.03 & 1.10 & 4.56 & 11.27 & 6.32 & 8.01 \\
\hline 7.607 & -2.235 & 527.31 & 419.08 & 419.10 & 6942.8 & 11.81 & 3467 & 0.664 & 1654 & 7.96 & 9.03 & 1.10 & 6.75 & 11.27 & 7.64 & 9.08 \\
\hline 10.173 & -2.223 & 563.78 & 459.34 & 459.35 & 6942.2 & 12.92 & 3256 & 0.664 & 1684 & 7.62 & 8.53 & 1.16 & 8.45 & 11.27 & 9.26 & 10.49 \\
\hline 12.700 & -2.223 & 594.62 & 497.62 & 497.64 & 6941.6 & 13.97 & 3082 & 0.664 & 1795 & 7.69 & 8.48 & 1.29 & 10.25 & 11.27 & 11.54 & 12.54 \\
\hline 2.540 & 2.197 & 432.49 & 340.18 & 340.19 & 6943.9 & 9.69 & 4012 & 0.663 & 1910 & 10.57 & 12.06 & 1.10 & 2.76 & 11.27 & 5.44 & 7.34 \\
\hline 5.080 & 2.223 & 480.28 & 378.91 & 378.92 & 6943.3 & 10.76 & 3731 & 0.663 & 1749 & 9.01 & 10.26 & 1.10 & 4.66 & 11.27 & 6.41 & 8.08 \\
\hline 7.620 & 2.223 & 526.43 & 418.71 & 418.73 & 6942.8 & 11.86 & 3486 & 0.664 & 1662 & 8.00 & 9.07 & 1.10 & 6.76 & 11.27 & 7.67 & 9.11 \\
\hline 10.147 & 2.223 & 566.98 & 458.17 & 458.19 & 6942.2 & 12.95 & 3278 & 0.664 & 1618 & 7.33 & 8.24 & 1.17 & 8.44 & 11.27 & 8.96 & 10.22 \\
\hline 12.713 & 2.223 & 595.26 & 496.87 & 496.88 & 6941.6 & 14.02 & 3100 & 0.664 & 1771 & 7.59 & 8.38 & 1.29 & 10.26 & 11.27 & 11.41 & 12.43 \\
\hline 7.607 & -3.493 & 539.80 & 425.65 & 425.66 & 6942.8 & 11.37 & 3253 & 0.664 & 1568 & 7.47 & 8.51 & 1.10 & 6.75 & 11.27 & 7.37 & 8.86 \\
\hline 7.633 & 3.493 & 541.48 & 426.74 & 426.75 & 6942.8 & 11.34 & 3230 & 0.664 & 1560 & 7.42 & 8.46 & 1.10 & 6.76 & 11.27 & 7.36 & 8.85 \\
\hline
\end{tabular}


Table 3 (continued)

Channel Specimen

Experiment 6

Date: 10 August 1990

Time: 14:25:15

$\begin{array}{cccccccc}\text { TA } & T 8 & M & P O & P 0-P 1 & V f & \text { Ot } & \text { Hqt } \\ K & K & \mathrm{~kg} / \mathrm{h} & \mathrm{KPa} & \mathrm{kPa} & \% & W & \% \\ 298.22 & 425.81 & 13.61 & 6950.0 & 9.28 & 25.27 & 2503.0 & 1.16\end{array}$

Hot-side Temperatures:

$\begin{array}{ccc}X & Y & T H \\ \mathrm{~cm} & \mathrm{~cm} & K \\ 1.588 & 0.655 & 367.00 \\ 2.540 & 0.655 & 375.62 \\ 5.080 & 0.655 & 396.82 \\ 7.620 & 0.655 & 419.16 \\ 10.160 & 0.655 & 435.80 \\ 12.700 & 0.655 & 450.41 \\ 13.653 & 0.655 & 457.23\end{array}$

Insulated-Side Temperatures and Calculated Data:

\begin{tabular}{|c|c|c|c|c|c|c|c|c|c|c|c|c|c|c|c|c|}
\hline $\begin{array}{c}\mathrm{x} \\
\mathrm{cm}\end{array}$ & $\begin{array}{c}Y \\
\mathrm{~cm}\end{array}$ & $\begin{array}{l}\text { TH } \\
K\end{array}$ & if & $\begin{array}{l}\text { Taw } \\
K\end{array}$ & $\begin{array}{l}P \\
k P a\end{array}$ & $\begin{array}{c}\mathrm{v} \\
\mathrm{m} / \mathrm{s}\end{array}$ & RE & PR & $\begin{array}{c}h \\
W /\left(m^{2} \cdot k\right)\end{array}$ & NU & Jm & $\begin{array}{l}\text { Jtw } \\
K\end{array}$ & $\begin{array}{l}\text { Wef } \\
k\end{array}$ & $\begin{array}{l}\text { Jre } \\
x\end{array}$ & th & $\%$ \\
\hline 00 & .965 & 4.71 & 8. & 21 & 50.0 & .53 & 9017 & 861 & 5524 & 33.32 & 2 & 1.10 & 0.84 & .27 & 43 & \\
\hline & & .24 & & & & & 18 & & & & & & 19 & 27 & 61 & \\
\hline 2.527 & 0 & 3.93 & 8.62 & 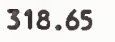 & 48.5 & .70 & 628 & 662 & 10 & & & 10 & .52 & .27 & .05 & \\
\hline .810 & 318 & 5.34 & 35 & & 4 & 5 & 56 & 662 & & & & & & & 58 & \\
\hline .080 & .318 & 5.49 & 8.08 & 11 & 466.9 & 1 & 92 & .662 & - & 0 & 0 & 0 & 52 & 27 & 7.25 & \\
\hline 363 & 18 & .06 & 2 & & & & & & & .98 & & & & 27 & 06 & \\
\hline .620 & & 3.6 & 8 & & & & 9 & & & & & & 63 & 27 & 91 & o. \\
\hline 8.903 & 305 & 3 & 8. & 07 & & & 331 & 3 & & & & & 07 & 27 & .57 & . \\
\hline 160 & 2 & 3 & 7 & & & & 693 & & & & & & & & 87 & 1. \\
\hline 3 & 8 & 35 & & & & & 63 & & & & & & & 27 & 28 & 3 . \\
\hline 700 & 35 & 0.41 & 6.95 & & & & 40 & & & & & & & 27 & .67 & \\
\hline .97 & 0.318 & 7 & 17.12 & & & 5 & 13 & 4 & & & & & & 27 & .90 & \\
\hline .240 & .953 & 1.26 & 05 & & & & 193 & & & 6 & & & & 27 & 30 & J. \\
\hline 2.52 & & - & 03 & & & & & & & & & & & & 18 & ?. \\
\hline .080 & 2.235 & 6.99 & 0.82 & & & . & 18 & 3 & & .96 & & & & 27 & 6.55 & . \\
\hline 7.607 & 2, 235 & 6.02 & 8 & & & & 1 & & & 0 & & & & 27 & 47 & \\
\hline 173 & & & & & & & 28 & & & & & & & & 51 & 1.6 \\
\hline 2.700 & -2.223 & 448.32 & 03.7 & & & & 382 & & & & & & 50 & 27 & 13.36 & 14.0 \\
\hline 2.540 & & 0.4 & 20.23 & & & & & & & & & & & & 5.89 & $.62-8$ \\
\hline 080 & 3 & & & & & & 880 & & & & & & & & 6.94 & 1 \\
\hline 620 & & & & & & & 7372 & & & & & & & & 8.45 & 9. \\
\hline .147 & & & 83.55 & & & & 194 & & & & & & & .27 & .69 & 1.7 \\
\hline .713 & 223 & 7.68 & 04.32 & & & & 844 & & & 1 & 59 & & .51 & .27 & 13.71 & 14.9 \\
\hline & & & & & & & & & & & & & 62 & & 8.00 & \\
\hline 633 & 3.493 & 25.86 & 68.64 & 368.66 & 0943.4 & 0.016 & 6652 & 0 & ket & 16.89 & 18.29 & 政 & .63 & .27 & 7.92 & 9. \\
\hline
\end{tabular}


Channel Specimen

Experiment 6

Date: 10 August 1990

Time: $14: 31: 20$

$\begin{array}{cccccccc}\text { TA } & \text { TB } & M & P O & P O-P 1 & V f & Q t & \text { Wqt } \\ K & K & \mathrm{~kg} / \mathrm{h} & \mathrm{KPa} & \mathrm{kPa} & \% & H & \% \\ 298.45 & 385.31 & 19.90 & 6956.2 & 16.79 & 25.27 & 2492.0 & 1.30\end{array}$

Hot-side Temperatures:

$\begin{array}{ccc}X & Y & T H \\ \mathrm{~cm} & \mathrm{~cm} & K \\ 1.588 & 0.655 & 350.58 \\ 2.540 & 0.655 & 355.99 \\ 5.080 & 0.655 & 370.11 \\ 7.620 & 0.655 & 385.14 \\ 10.160 & 0.655 & 396.47 \\ 12.700 & 0.655 & 406.89 \\ 13.653 & 0.655 & 412.13\end{array}$

Insulated-Side Temperatures and Calculated Data:

\begin{tabular}{|c|c|c|c|c|c|c|c|c|c|c|c|c|c|c|c|c|}
\hline$x$ & $Y$ & $T H$ & Tf & Taw & $\mathbf{P}$ & v & RE & PR & h & NU & Num & HtH & Wtf & Hre & Wh & Wnu \\
\hline $\mathrm{cm}$ & $\mathrm{cm}$ & $k$ & $k$ & $K$ & $\mathrm{kPa}$ & $\mathrm{m} / \mathrm{s}$ & & & $H /\left(m^{2} \cdot k\right)$ & & & $k$ & $k$ & $x$ & $x$ & $\%$ \\
\hline 0.000 & -0.965 & 16.43 & 298.38 & 298.44 & 6956.2 & 25.96 & 13351 & 0.661 & 8095 & 48.80 & 50.40 & 1.10 & 0.68 & 11.27 & 13.39 & 14.26 \\
\hline 1.270 & -0.318 & 335.60 & 305.25 & 305.31 & 6954.8 & 26.55 & 13152 & 0.662 & 6589 & 39.15 & 41.24 & 1.10 & 0.88 & 1.27 & 6.20 & 7.91 \\
\hline 2.527 & -0.330 & 344.78 & 312.11 & 312.17 & 6953.4 & 27.13 & 12958 & 0.662 & 5542 & 32.45 & 34.28 & 1.10 & 1.08 & 11.27 & 6.49 & 8.14 \\
\hline 3.810 & -0.318 & 352.46 & 318.65 & 318.71 & 6952.0 & 27.68 & 12780 & 0.662 & 5241 & 30.27 & 32.00 & 1.10 & 1.38 & 11.27 & 6.90 & 8.48 \\
\hline 5.080 & -0.318 & 359.22 & 325.19 & 325.25 & 6950.6 & 28.24 & 12608 & 0.662 & 5348 & 30.48 & 32.20 & 1.10 & 1.73 & 11.27 & 7.48 & 8.95 \\
\hline 6.363 & -0.318 & 366.29 & 331.93 & 332.00 & 6949.2 & 28.81 & 12435 & 0.662 & 5357 & 30.12 & 31.80 & 1.10 & 2.11 & 11.27 & 8.19 & 9.55 \\
\hline 7.620 & -0.330 & 373.40 & 338.56 & 338.63 & 6947.8 & 29.38 & 12271 & 0.662 & 5274 & 29.27 & 30.89 & 1.10 & 2.48 & 11.27 & 8.95 & 10.21 \\
\hline 8.903 & -0.305 & 380.94 & 345.31 & 345.39 & 6946.4 & 29.95 & 12109 & 0.663 & 5146 & 28.19 & 29.75 & 1.10 & 2.78 & 11.27 & 9.48 & 10.68 \\
\hline 10.160 & $\cdot 0.318$ & 385.76 & 351.88 & 351.96 & 6945.0 & 30.51 & 11957 & 0.663 & 5331 & 28.84 & 30.34 & 1.10 & 3.10 & 11.27 & 10.68 & 11.76 \\
\hline 1.430 & -0.318 & 391.18 & 358.35 & 358.43 & 6943.6 & 31.06 & 11811 & 0.663 & 5340 & 28.54 & 29.95 & 1.10 & 3.42 & 11.27 & 11.88 & 12.85 \\
\hline 2.700 & -0.305 & 396.88 & 364.74 & 364.83 & 6942.2 & 31.60 & 11670 & 0.663 & 5563 & 29.39 & 30.79 & 1.10 & 3.76 & 11.27 & 13.01 & 13.91 \\
\hline 3.970 & -0.318 & 402.21 & 371.57 & 371.66 & 6940.8 & 32.19 & 11525 & 0.663 & 6432 & 33.55 & 35.04 & 1.10 & 4.13 & 11.27 & 14.60 & 15.41 \\
\hline 15.240 & -0.953 & 397.33 & 378.25 & 378.34 & 6939.4 & 32.75 & 11387 & 0.663 & 7174 & 36.98 & 37.99 & 1.10 & 4.48 & 11.27 & 27.39 & 27.83 \\
\hline 5.080 & -2.235 & 370.28 & 327.54 & 327.59 & 6950.6 & 26.15 & 11540 & 0.662 & 4255 & 24.14 & 25.82 & 1.10 & 1.73 & 11.27 & 6.52 & 8.17 \\
\hline 7.607 & -2.235 & 379.87 & 342.00 & 342.06 & 6947.8 & 27.28 & 11210 & 0.663 & 4850 & 26.74 & 28.33 & 1.10 & 2.48 & 11.27 & 8.40 & 9.73 \\
\hline 10.173 & -2.223 & 391.80 & 356.63 & 356.70 & 6945.0 & 28.43 & 10898 & 0.663 & 5133 & 27.52 & 28.98 & 1.10 & 3.10 & 11.27 & 10.37 & 11.47 \\
\hline 12.700 & -2.223 & 403.26 & 370.55 & 370.62 & 6942.2 & 29.52 & 10620 & 0.663 & 5464 & 28.56 & 29.92 & 1.10 & 3.76 & 11.27 & 12.81 & 13.72 \\
\hline 2.540 & 2.197 & 348.15 & 313.21 & 313.26 & 6953.4 & 25.34 & 12038 & 0.662 & 5176 & 30.24 & 32.05 & 1.10 & 1.08 & 11.27 & 6.27 & 7.97 \\
\hline 5.080 & 2.223 & 363.98 & 327.18 & 327.24 & 6950.6 & 26.45 & 11691 & 0.662 & 4944 & 28.07 & 29.76 & 1.10 & 1.73 & 11.27 & 7.11 & 8.65 \\
\hline 7.620 & 2.223 & 378.94 & 341.54 & 341.60 & 6947.8 & 27.58 & 11359 & 0.663 & 4911 & 27.10 & 28.69 & 1.10 & 2.48 & 11.27 & 8.48 & 9.81 \\
\hline 10.147 & 2.223 & 391.13 & 355.77 & 355.84 & 6945.0 & 28.71 & 11051 & 0.663 & 5109 & 27.44 & 28.91 & 1.10 & 3.09 & 11.27 & 10.31 & 11.42 \\
\hline 12.713 & 2.223 & 402.78 & 369.73 & 369.81 & 6942.2 & 29.82 & 10767 & 0.663 & 5413 & 28.33 & 29.70 & 1.10 & 3.76 & 11.27 & 12.71 & 13.63 \\
\hline 7.607 & -3.493 & 389.89 & 347.38 & 347.43 & 6947.8 & 24.67 & 9879 & 0.663 & 4319 & 23.56 & 25.10 & 1.10 & 2.48 & 11.27 & 7.74 & 9.17 \\
\hline 7.633 & 3.493 & 388.35 & 346.64 & 346.70 & 6947.8 & 25.08 & 10076 & 0.663 & 4402 & 24.05 & 25.60 & 1.10 & 2.49 & 11.27 & 7.85 & 9.26 \\
\hline
\end{tabular}


Channel specimen

Experiment 6

Date: 10 August 1990

Time: 14:36:47

$\begin{array}{cccccccc}T A & T B & M & P O & P O-P q & V f & \text { ot } & \text { Wqt } \\ K & K & \mathrm{~kg} / \mathrm{h} & \mathrm{kPa} & \mathrm{kPg} & \boldsymbol{X} & \mathrm{H} & \mathrm{X}\end{array}$

$\begin{array}{llllllll}298.61 & 358.80 & 28.62 & 6961.4 & 30.17 & 25.28 & 2482.0 & 1.56\end{array}$

Hot-side Temperatures:

$\begin{array}{ccc}X & Y & T W \\ \mathrm{~cm} & \mathrm{~cm} & K \\ 1.588 & 0.655 & 339.50 \\ 2.540 & 0.655 & 342.89 \\ 5.080 & 0.655 & 352.57 \\ 7.620 & 0.655 & 362.95 \\ 10.160 & 0.655 & 370.69 \\ 12.700 & 0.655 & 377.89 \\ 13.653 & 0.655 & 382.00\end{array}$

Insulated-Side Temperatures and Calculated Data:

\begin{tabular}{|c|c|c|c|c|c|c|c|c|c|c|c|c|c|c|c|c|}
\hline$x$ & $Y$ & Tw & Tf & Taw & P & v & RE & PR & h & WU & Num & Htw & Wtf & Wre & Wh & Unu \\
\hline $\mathrm{cm}$ & $\mathrm{cm}$ & K & $K$ & $K$ & $\mathrm{kPa}$ & $\mathrm{m} / \mathrm{s}$ & & & $H /\left(m^{2} \cdot K\right)$ & & & $k$ & $k$ & $x$ & $x$ & $\%$ \\
\hline 0.000 & -0.965 & 311.15 & 298.48 & 298.60 & 6961.4 & 37.81 & 19448 & 0.661 & 11560 & 69.68 & 71.29 & 1.10 & 0.59 & 11.27 & 15.08 & 15.86 \\
\hline 1.270 & -0.318 & 325.78 & 03.18 & 303.30 & 6958.9 & 38.40 & 19249 & 0.662 & 8845 & 52.78 & 54.91 & 1.10 & 0.69 & 11.27 & 7.14 & 8.67 \\
\hline 2.527 & -0.330 & 332.08 & 307.86 & 307.99 & 6956.4 & 38.99 & 19053 & 0.662 & 7474 & 44.16 & 46.04 & 1.10 & 0.81 & 11.27 & 7.25 & 8.76 \\
\hline 3.810 & -0.318 & 337.42 & 312.33 & 12.46 & 6953.9 & 39.55 & 18871 & 0.662 & 7062 & 41.33 & 43.12 & 1.10 & 1.00 & 11.27 & 7.51 & 8.98 \\
\hline 5.080 & -0.318 & 341.99 & 316.80 & 316.94 & 6951.4 & 40.11 & 18693 & 0.662 & 7221 & 41.87 & 43.67 & 1.10 & 1.23 & 11.27 & 7.97 & 9.36 \\
\hline 6.363 & -0.318 & 346.84 & 321.41 & 321.55 & 6948.8 & 40.69 & 18513 & 0.662 & 7236 & 41.56 & 43.34 & 1.10 & 1.48 & 11.27 & 8.54 & 9.86 \\
\hline 7.620 & -0.330 & 351.71 & 325.94 & 326.08 & 6946.3 & 41.26 & 18340 & 0.662 & 7128 & 40.56 & 42.29 & 1.10 & 1.74 & 11.27 & 9.18 & 10.41 \\
\hline 8.903 & -0.305 & 357.19 & 330.56 & 330.70 & 6943.8 & 41.84 & 18168 & 0.662 & 6882 & 38.81 & 40.50 & 1.10 & 1.95 & 11.27 & 9.54 & 10.73 \\
\hline 10.160 & -0.318 & 360.20 & 335.05 & 335.20 & 6941.3 & 42.41 & 18005 & 0.662 & 7180 & 40.13 & 41.76 & 1.10 & 2.16 & 11.27 & 10.70 & 11.78 \\
\hline 11.430 & -0.318 & 364.05 & 339.47 & 339.63 & 6938.8 & 42.97 & 17847 & 0.662 & 7132 & 39.51 & 41.06 & 1.10 & 2.39 & 11.27 & 11.71 & 12.70 \\
\hline 12.700 & -0.305 & 368.03 & 343.84 & 344.00 & 6936.3 & 43.52 & 17694 & 0.663 & 7394 & 40.62 & 42.17 & 1.10 & 2.62 & 11.27 & 12.68 & 13.60 \\
\hline 13.970 & -0.318 & 372.15 & 348.51 & 348.67 & 6933.8 & 44.10 & 17534 & 0.663 & 8338 & 45.40 & 47.07 & 1.10 & 2.88 & 11.27 & 13.81 & 14.66 \\
\hline 15.240 & -0.953 & 368.09 & 353.07 & 353.24 & 6931.3 & 44.68 & 17381 & 0.663 & 9141 & 49.34 & 50.48 & 1.10 & 3.12 & 11.27 & 25.67 & 26.14 \\
\hline 5.080 & -2.235 & 352.74 & 318.75 & 318.86 & 6951.4 & 36.53 & 16853 & 0.662 & 5340 & 30.84 & 32.61 & 1.10 & 1.23 & 11.27 & 6.62 & 8.25 \\
\hline 7.607 & -2.235 & 357.22 & 328.79 & 328.91 & 6946.4 & 37.67 & 16506 & 0.662 & 6452 & 36.51 & 38.21 & 1.10 & 1.74 & 11.27 & 8.51 & 9.83 \\
\hline 10.173 & -2.223 & 364.77 & 338.95 & 339.08 & 6941.3 & 38.82 & 16173 & 0.662 & 6986 & 38.74 & 40.34 & 1.10 & 2.16 & 11.27 & 10.47 & 11.57 \\
\hline 12.700 & -2.223 & 372.83 & 348.62 & 348.75 & 6936.3 & 39.92 & 15870 & 0.663 & 7377 & 40.16 & 41.67 & 1.10 & 2.62 & 11.27 & 12.66 & 13.58 \\
\hline 2.540 & 2.197 & 334.57 & 308.65 & 308.77 & 6956.4 & 36.30 & 17666 & 0.662 & 6974 & 41.13 & 42.99 & 1.10 & 0.81 & 11.27 & 6.97 & 8.53 \\
\hline 5.080 & 2.223 & 345.49 & 318.23 & 318.35 & 6951.4 & .42 & 17309 & 0.662 & 6666 & 38.54 & 40.32 & 1.10 & 1.23 & 11.27 & 7.55 & 9.01 \\
\hline 7.620 & 2.223 & 355.79 & 328.07 & .20 & 6946.3 & 38.56 & 16960 & 0.662 & 6621 & 37.52 & 39.23 & 1.10 & 9.74 & 11.27 & 8.68 & 9.98 \\
\hline 10.147 & 2.223 & 364.29 & 337.83 & 337.96 & 6941.3 & 39.70 & 16630 & 0.662 & 6819 & 37.90 & 39.51 & 1.10 & 2.16 & 11.27 & 10.25 & 11.37 \\
\hline 12.713 & 2.223 & 372.51 & 347.39 & 347.53 & 6936.3 & 40.82 & 16320 & 0.663 & 7119 & 38.84 & 40.36 & 1.10 & 2.62 & 11.27 & 12.27 & 13.22 \\
\hline 7.607 & -3.493 & 367.14 & 333.95 & 334.04 & 6946.4 & 32.71 & 13972 & 0.662 & 5519 & 30.91 & 32.56 & 1.10 & 1.74 & 11.27 & 7.63 & 9.08 \\
\hline 7.633 & 3.493 & 363.66 & 332.21 & 332.31 & 6946.3 & 34.33 & 14793 & 0.662 & 5827 & 32.75 & 34.42 & 1.10 & 1.74 & 11.27 & 7.93 & 9.33 \\
\hline
\end{tabular}


Channel specimen

Experiment 6

Date: 10 August 1990

Time: $14: 41: 44$

$\begin{array}{cccccccc}\text { TA } & T 8 & M & P O & P 0-P 1 & V f & \text { Ot } & \text { Wqt } \\ K & K & \mathrm{~kg} / \mathrm{h} & \mathrm{KPa} & \mathrm{kPa} & \mathrm{X} & \mathrm{H} & \mathrm{K} \\ 298.58 & 340.38 & 40.98 & 6966.2 & 55.92 & 25.30 & 2467.0 & 1.98\end{array}$

Hot-side Temperatures:

$\begin{array}{ccc}X & Y & T H \\ \mathrm{~cm} & \mathrm{~cm} & K \\ 1.588 & 0.655 & 331.19 \\ 2.540 & 0.655 & 333.25 \\ 5.080 & 0.655 & 339.95 \\ 7.620 & 0.655 & 347.15 \\ 10.160 & 0.655 & 352.33 \\ 12.700 & 0.655 & 357.29 \\ 13.653 & 0.655 & 360.51\end{array}$

Insulated-Side Temperatures and Calculated Data:

\begin{tabular}{|c|c|c|c|c|c|c|c|c|c|c|c|c|c|c|c|c|}
\hline & & & & & & & & & & & & & & & & \\
\hline$x$ & $Y$ & Tw & Tf & Tan & $P$ & $v$ & RE & PR & h & NU & NUm & $H t w$ & Wtf & Wre & Wh & Unu \\
\hline $\mathrm{cm}$ & $\mathrm{cm}$ & K & K & K & kPa & $\mathrm{m} / \mathrm{s}$ & & & $H /\left(m^{2} \cdot K\right)$ & & & $k$ & $k$ & $x$ & $x$ & $x$ \\
\hline 0.000 & -0.965 & 307.38 & 298.30 & 298.55 & 6966.2 & 54.89 & 28276 & 0.661 & 16335 & 98.49 & 100.13 & 1.10 & 0.55 & 11.27 & 17.98 & 18.64 \\
\hline 1.270 & -0.318 & 318.49 & 01.51 & 1.77 & 6961.6 & .49 & 28080 & 662 & 18 & .78 & 72.95 & .10 & 0.58 & .27 & 8.63 & 9.93 \\
\hline 2.527 & -0.330 & 322.84 & 304.71 & 304.98 & 6957.0 & .10 & 27883 & 0.662 & 10018 & .59 & 61.51 & 1.10 & 0.64 & 27 & 8.52 & 9.84 \\
\hline 3.810 & -0.318 & 326.64 & 307.77 & 308.04 & 6952.3 & 6.68 & 27698 & 0.662 & 9416 & .64 & 57.49 & 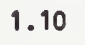 & $0 . \pi 3$ & 7 & 8.58 & 9.89 \\
\hline 5.080 & -0.318 & 329.71 & 10.82 & 311.10 & 6947.6 & .26 & 27517 & 0.662 & 9661 & 2 & 9 & 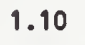 & .90 & 7 & 8.92 & 0.19 \\
\hline 7.620 & -0.330 & 336.38 & 17.07 & 317.35 & 6938.3 & .45 & 27154 & 0.662 & 9545 & 32 & 5 & ) & .23 & 7 & 9.83 & 1.00 \\
\hline 8.903 & -0.305 & 340.39 & 320.22 & 320.51 & 6933.6 & .05 & 26975 & 0.662 & 9114 & .48 & 7 & & .38 & 7 & 9.99 & 1.13 \\
\hline 0.160 & -0.318 & 342.21 & 323.29 & 323.59 & 6929.0 & 59.64 & 26803 & 0.662 & 9584 & 84 & 8 & & 1.52 & 7 & 1.13 & 12.16 \\
\hline 1.430 & -0.318 & 344.89 & 326.31 & 326.62 & 6924.3 & 60.22 & 26636 & 0.662 & 9477 & 53.90 & 55.57 & 1 . & 1.68 & 27 & 11.99 & 12.96 \\
\hline 12.700 & -0.305 & 347.61 & 329.30 & 329.61 & 6919.6 & 60.79 & 26475 & 0.662 & 9809 & 55.45 & 57.13 & 1.10 & 1.84 & 27 & 12.83 & 13.75 \\
\hline 13.970 & -0.318 & 350.81 & 332.49 & 332.81 & 6915.0 & 61.40 & 26304 & 0.662 & 10807 & 60.70 & 62.52 & 1.10 & 2.03 & 27 & 13.55 & 14.42 \\
\hline 15.240 & -0.953 & 347.48 & 335.61 & 335.93 & 6910.3 & 62.00 & 26140 & 0.662 & 11680 & 65.21 & 66.47 & 1.10 & 2.20 & 27 & 24.82 & 25.30 \\
\hline 5.080 & -2.235 & 340.22 & 312.45 & 312.67 & 6947.6 & 51.16 & 24375 & 0.662 & 6528 & 38.20 & 40.03 & 1.10 & 0.90 & .27 & 6.93 & 8.50 \\
\hline 7.607 & -2.235 & 341.07 & 319.44 & 319.67 & 6938.3 & 52.33 & 24017 & 0.662 & 8487 & 48.95 & 50.75 & 1.10 & 1.23 & 11.27 & 8.99 & 10.25 \\
\hline 10.173 & -2.223 & 345.55 & 326.52 & 326.76 & 6928.9 & 53.53 & 23668 & 0.662 & 9494 & 53.97 & 55.68 & 1.10 & 1.53 & .27 & 11.05 & 12.09 \\
\hline 12.700 & -2.223 & 351.12 & 333.25 & 333.50 & 6919.6 & 54. & 23346 & 0.662 & 10 & 56.21 & 57.85 & 1. & 1.84 & .27 & 13.08 & 13.97 \\
\hline 2.540 & 2.197 & 324.69 & 305.30 & 305.53 & 6956.9 & & 25772 & 0.662 & & & & & .64 & 1.27 & 8.13 & 9.50 \\
\hline 5.080 & 2.223 & 332.32 & 311.87 & 312.11 & 6947.6 & 53 & 25 & 0.662 & 88 & 13 & & & .90 & 1.27 & 8.41 & 9.75 \\
\hline 7.620 & 2.223 & 339.42 & 318.63 & 318.87 & 6938.3 & 54.35 & 25048 & 0.662 & 88 & 05 & 52.86 & 1.10 & .23 & 1.27 & 9.27 & 10.49 \\
\hline 10.147 & 2.223 & 345.28 & 325.32 & 325.58 & 6929.0 & & 24702 & 0.662 & 90 & 61 & 53.33 & 1.10 & .52 & 1.27 & 10.62 & 1.70 \\
\hline 12.713 & 2.223 & 351.01 & 331.88 & 332.15 & 6919.6 & 56.69 & 24374 & 0.662 & 9368 & 52.69 & 54.34 & 1.10 & 1.85 & 1.27 & 12.34 & 13.28 \\
\hline 7.607 & -3.493 & 350.68 & 324.30 & 324.46 & 6938.3 & 43.31 & 19391 & 0.662 & 6924 & 39.53 & & 1.10 & 1.23 & 11.27 & 7.80 & 9.22 \\
\hline 7.633 & 3.493 & 345.96 & 321.97 & 322.16 & 6938.2 & 47.35 & 21452 & 0.662 & 7628 & 43.76 & 45.52 & 1.10 & 1.24 & 11.27 & 8.34 & 9.68 \\
\hline
\end{tabular}


Table 3 (continued)

Channel Specinen

Experiment 7

Date: 9 August 1990

Time: $14: 34: 42$

$\begin{array}{cccccccc}\text { TA } & T B & \mathrm{M} & \mathrm{PO} & \mathrm{PO}-\mathrm{P1} & \mathrm{Vf} & \text { ot } & \text { Wqt } \\ \mathrm{K} & \mathrm{K} & \mathrm{kg} / \mathrm{h} & \mathrm{KPa} & \mathrm{KPa} & \mathrm{X} & \mathrm{H} & \mathrm{X} \\ 299.11 & 665.42 & 9.27 & 6956.4 & 6.81 & 50.71 & 4898.0 & 1.04\end{array}$

Hot-side Temperatures:

$\begin{array}{ccc}X & Y & T H \\ \mathrm{~cm} & \mathrm{~cm} & K \\ 1.588 & 0.655 & 495.77 \\ 2.540 & 0.655 & 525.72 \\ 5.080 & 0.655 & 593.97 \\ 7.620 & 0.655 & 664.19 \\ 10.160 & 0.655 & 732.79 \\ 12.700 & 0.655 & 778.08 \\ 13.653 & 0.655 & 783.58\end{array}$

Insulated-Side Temperatures and Calculated Data:

\begin{tabular}{|c|c|c|c|c|c|c|c|c|c|c|c|c|c|c|c|c|}
\hline & & & & & & & & & & & & & & . & 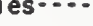 & \\
\hline$x$ & $Y$ & $w$ & Tf & Taw & $P$ & $v$ & RE & PR & h & IU & NUm & Wtw & Hef & Wre & Wh & Wnu \\
\hline $\mathrm{cm}$ & $\mathrm{cm}$ & $k$ & $k$ & $k$ & $\mathrm{kPa}$ & & & & $\mathrm{H} /\left(\mathrm{m}^{2}\right.$. & & & $k$ & $x$ & $x$ & $x$ & $\%$ \\
\hline 0.000 & .965 & 31.04 & $\times 9.10$ & 9.11 & 956.4 & .87 & 078 & .661 & 3495 & 1.04 & & 1.10 & 1.99 & 1.27 & 1.61 & 6.01 \\
\hline 1.270 & 0.318 & 5.45 & 28.73 & 8.74 & 955.8 & & 707 & .662 & 3096 & .52 & 0.96 & 1.10 & 3.19 & & & .8 \\
\hline 2.527 & -0.330 & א & 8.30 & 58.32 & 5.3 & & 86 & .663 & 88 & 3.30 & 6.00 & .10 & 4.21 & 27 & & .2 \\
\hline 3.810 & -0.318 & 7.67 & 6.48 & 36.50 & 4.7 & 2 & 17 & .663 & 00 & 9 & 4.02 & .10 & 5.61 & 27 & & .62 \\
\hline 5.080 & -0.318 & 70.24 & 14.68 & 4.70 & 954.1 & .30 & 178 & .664 & 5 & 2 & 3.25 & 19 & 7.16 & 27 & 36 & .04 \\
\hline 6.363 & -0.318 & 03.71 & 3.76 & 3.78 & 6953.6 & .42 & 57 & 0.664 & 257 & 0.45 & 38 & .32 & 8.77 & 27 & & 8.56 \\
\hline 7.620 & -0.330 & 37.71 & 472.33 & .36 & 3.0 & 1 & 62 & 0.664 & 180 & 9.67 & 1.41 & 1.46 & 10.38 & & & 9.10 \\
\hline 8.903 & -0.305 & 72.79 & 501.45 & 01.48 & 6052 & 7 & 82 & 0.664 & 099 & 8.94 & 0.51 & 1.60 & 1.65 & & & .47 \\
\hline .160 & -0.318 & 03.37 & 29.77 & 9.80 & 0 & 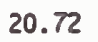 & 23 & 0.664 & 2041 & 837 & 9.78 & 1.72 & 2.97 & 7 & 1 & 0.00 \\
\hline .430 & -0.318 & 29.94 & 557.68 & $?$ & 10 & 1.79 & 9 & 0.664 & 26 & 7.90 & 9.16 & 3 & 4.34 & & & 0.71 \\
\hline .700 & -0.305 & 751.34 & 585.23 & 5.28 & 60 & 85 & 849 & 0.665 & 11 & 8.08 & 9.27 & 1 & 5.75 & & & 1.62 \\
\hline .970 & -0.318 & 758.92 & 614.70 & 14.74 & 6950.2 & .98 & 720 & 0.665 & 2679 & 9.91 & 1.13 & 1.94 & 17.33 & & & 3.68 \\
\hline .240 & -0.953 & 28.54 & 643.47 & 43.53 & 6949.6 & .09 & 603 & 0.665 & & 11.30 & 2.10 & .82 & 18.77 & & & 26.02 \\
\hline 2.527 & -2.223 & 17.10 & 62.92 & 62.93 & 6955.3 & .28 & 953 & 0.663 & 14 & 12.21 & 4.83 & & 4.21 & & & 7.16 \\
\hline 5.080 & -2.235 & 92.53 & 423.69 & 23.71 & 6954.1 & .44 & 459 & 0.664 & 15 & 10.10 & 12.15 & & 7.16 & & & 7.84 \\
\hline 7.607 & -2.235 & 63.79 & 485.52 & 85.55 & 6953.0 & .64 & 062 & 0.664 & 2022 & 8.80 & 10.45 & & 0.36 & & & 8.78 \\
\hline .173 & -2.223 & 22.25 & 548.07 & 48.10 & 6951.9 & .87 & 736 & 0.664 & & 8.15 & 9.49 & 1.80 & 2.99 & & & .99 \\
\hline 2.700 & -2.223 & 71.36 & 607.55 & 07.59 & & & 3479 & 0.665 & & 7.99 & 9.11 & & 15.75 & & 6 & 11.74 \\
\hline 2.540 & 2.197 & 09.39 & & & & & 084 & 0.663 & & 2.77 & .42 & & 4.22 & & & 7.22 \\
\hline 5.080 & 2.223 & 80.47 & 420.76 & 20.78 & 6954.1 & & 588 & 0.664 & 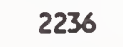 & 10.73 & .81 & & 7.16 & & 7 & .97 \\
\hline 7.620 & 2.223 & 55.52 & 481.44 & 81.47 & & & 184 & 0.664 & & & & & 0.38 & & & .88 \\
\hline .147 & & 22.61 & 541.59 & & & & 8858 & 0.664 & & & & & 12.96 & & & 9.77 \\
\hline 12.713 & 2.223 & 767.47 & 600.59 & 600.63 & & & 3591 & 0.665 & & & 9.05 & & 15.77 & & & 11.60 \\
\hline 7.607 & -3.493 & 685.30 & 496.52 & 496.54 & & & $3 m 7$ & & & & & & 10.36 & & & 8.57 \\
\hline 7.633 & 3.493 & 683.69 & 496.03 & 496.05 & 6953.0 & 17.11 & 3802 & 0.664 & 1921 & 8.24 & 9.83 & 1.64 & 10.39 & 11.27 & 7.06 & 8.60 \\
\hline
\end{tabular}


Channel Specimen

Experiment 7

Date: 9 August 1990

Time: $14: 42: 56$

$\begin{array}{cccccccc}T A & T B & M & P O & P O-P 1 & V f & \text { Qt } & \text { Wat } \\ K & K & \mathrm{~kg} / \mathrm{h} & \mathrm{KPa} & \mathrm{KPa} & \boldsymbol{*} & W & \% \\ 299.44 & 521.66 & 15.90 & 6950.9 & 14.27 & 50.78 & 5094.0 & 1.07\end{array}$

Hot-side Temperatures:

$\begin{array}{ccc}X & Y & T w \\ \mathrm{~cm} & \mathrm{~cm} & K \\ 1.588 & 0.655 & 426.44 \\ 2.540 & 0.655 & 441.36 \\ 5.080 & 0.655 & 477.89 \\ 7.620 & 0.655 & 517.14 \\ 10.160 & 0.655 & 545.65 \\ 12.700 & 0.655 & 570.98 \\ 13.653 & 0.655 & 584.19\end{array}$

Insulated-Side Temperatures and Calculated Data:

\begin{tabular}{|c|c|c|c|c|c|c|c|c|c|c|c|c|c|c|c|c|}
\hline$x$ & $Y$ & $T_{H}$ & $T f$ & Taw & $P$ & v & RE & PR & h & NU & Num & Het & Utf & Hre & the & Unu \\
\hline $\mathrm{cm}$ & $\mathrm{cm}$ & K & K & $K$ & $\mathrm{kPa}$ & $\mathrm{m} / \mathrm{s}$ & & & $H /\left(m^{2} \cdot k\right)$ & & & $k$ & K & $x$ & $x$ & $\%$ \\
\hline 0.000 & 0.965 & 5.77 & 99.40 & 99.44 & 6950.9 & 20.58 & 10515 & 662 & 6429 & 38.68 & 1.87 & 1.10 & 1.27 & 1.27 & 1.84 & 2.82 \\
\hline 1.270 & 18 & 2.93 & 7.21 & 7.25 & 7 & .76 & 118 & 662 & 392 & 1.24 & 5.14 & 10 & 1.97 & & & 7.02 \\
\hline 2.527 & 0.330 & 7.38 & 34.97 & 35.02 & 6948.5 & 2.94 & 9755 & .662 & 487 & 5.08 & 8.31 & 1.10 & 2.57 & & 55 & 7.42 \\
\hline 3.810 & 0.318 & 36.77 & 51.91 & 51.96 & 6947.3 & 24.07 & 9436 & .663 & 4264 & 23.07 & 25.98 & 1.10 & 3.42 & .27 & 13 & 7.86 \\
\hline 5.080 & 0.318 & 54.27 & 368.85 & 68.90 & 46.1 & 25.20 & 9142 & 0.663 & 4350 & 22.81 & 25.58 & 0 & 4.35 & 7 & & 8.40 \\
\hline 6.363 & -0.318 & 472.43 & 386.32 & 386.37 & 6944.9 & 26.37 & 8860 & 0.663 & 4363 & 22.18 & 24.78 & .10 & 5.33 & 7 & 4 & .09 \\
\hline 7.620 & -0.330 & 490.79 & 03.49 & 403.55 & 6943.7 & 27.51 & 8602 & 0.663 & 4297 & 21.21 & 23.62 & .10 & 6.30 & 7 & 50 & 9.82 \\
\hline 8.903 & -0.305 & 509.12 & 420.98 & 421.05 & 6942.5 & 28.68 & 8356 & 0.664 & 4247 & 20.37 & 22.62 & 1.10 & 7.07 & 27 & 9.19 & 0.43 \\
\hline 0.160 & -0.318 & 521.69 & 437.99 & 438.07 & 6941.4 & 29.82 & 8133 & 0.664 & 4406 & 20.58 & 22.66 & 1.10 & 7.88 & 1.27 & 0.46 & 11.56 \\
\hline 1.430 & -0.318 & 534.64 & 454.76 & 454.85 & 0.2 & 30.93 & 7927 & 0.664 & 4481 & 20.40 & 22.30 & 1.10 & 8.70 & 27 & 87 & 12.85 \\
\hline 12.700 & -0.305 & 548.83 & 471.32 & 471.40 & 6939.0 & 32.04 & 7735 & 0.664 & 1700 & 20.92 & 22.75 & 1.10 & 9.56 & 27 & & 4.08 \\
\hline 3.970 & -0.318 & 561.58 & 489.02 & 489.11 & 78 & 33.22 & 7541 & 0.664 & 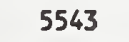 & 24.02 & 25.92 & 1.15 & 10.52 & 27 & & 5.94 \\
\hline 15.240 & -0.953 & 0.64 & 506.31 & 41 & 6 & 3 & 63 & 0.664 & 6300 & .65 & .91 & 1.11 & 11.39 & & 28 & 29.24 \\
\hline 5.080 & -2.235 & 470 & 73.79 & 34 & 6 & & 58 & 33 & 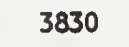 & 0 & 22.59 & 1.10 & 4.35 & & & 8.02 \\
\hline 7.607 & -2.235 & 3 & 1 & & & & 34 & 54 & & & 21.90 & & 6.29 & & 7 & 9.45 \\
\hline 10.173 & -2.223 & & & & & & 6 & & & & 20.99 & & 7.88 & & & 11.08 \\
\hline 12.700 & -2.223 & & & & & & 5 & 54 & & 19.64 & 21.39 & & 9.56 & & & 13.60 \\
\hline 2.540 & 2.197 & 425.17 & 337.79 & & 6948.5 & & 9033 & 0.662 & & 23.50 & 26.67 & & 2.58 & & & 7.33 \\
\hline 5.080 & 2.223 & 465.13 & 373.98 & 374.03 & 6946.1 & 23.79 & 8434 & 0.663 & 6 & 21.17 & 23.87 & & 4.35 & 1.27 & 36 & 8.20 \\
\hline 7.620 & 2.223 & 504.92 & 411.18 & 411.24 & 6943.7 & 26.10 & 7908 & 0.664 & 002 & 19.50 & 21.83 & & 6.30 & 7 & .07 & 9.45 \\
\hline 10.147 & 2.223 & 533.47 & 448.05 & 448.12 & 6941.4 & 28.39 & 7457 & 0.664 & 4318 & 19.86 & 21.86 & 1.10 & 7.87 & 11.27 & 10.28 & 11.40 \\
\hline 12.713 & 2.223 & 562.00 & 484.21 & 484.29 & 6939.0 & 30.64 & 7071 & 0.664 & 4696 & 20.48 & 22.23 & 1.15 & 9.57 & 11.27 & 13.17 & 14.06 \\
\hline 7.607 & -3.493 & 518.68 & 418.56 & 418.61 & 6943.8 & 24.87 & 7317 & 0.664 & 3747 & 18.04 & 20.30 & 1.10 & 6.29 & 11.27 & 7.69 & 9.13 \\
\hline 7.633 & 3.493 & 525.98 & 422.77 & 422.82 & 6943.7 & 24.34 & 7043 & 0.664 & 3634 & 17.38 & 19.60 & 1.10 & 6.31 & 11.27 & 7.55 & 9.01 \\
\hline
\end{tabular}


Table 3 (continued)

Channel specimen

Experiment 7

Date: 9 August 1990

Time: 14:49:58

$\begin{array}{cccccccc}\text { TA } & T B & \mathrm{M} & \mathrm{PO} & \mathrm{PO} P 1 & V f & \text { Qt } & \text { Hqt } \\ \mathrm{K} & \mathrm{K} & \mathrm{kg} / \mathrm{h} & \mathrm{KPa} & \mathrm{KPa} & \times & W & X \\ 299.11 & 460.12 & 21.95 & 6963.6 & 23.25 & 50.80 & 5095.0 & 1.11\end{array}$

Hot-side Temperatures:

$\begin{array}{ccc}X & Y & T w \\ c m & c m & K \\ 1.588 & 0.655 & 400.02 \\ 2.540 & 0.655 & 410.01 \\ 5.080 & 0.655 & 436.21 \\ 7.620 & 0.655 & 464.28 \\ 10.160 & 0.655 & 485.08 \\ 12.700 & 0.655 & 504.25 \\ 13.653 & 0.655 & 514.57\end{array}$

Insulated-Side Temperatures and Calculated Data:

\begin{tabular}{|c|c|c|c|c|c|c|c|c|c|c|c|c|c|c|c|c|}
\hline & & & & & & & & & & & & & & . & 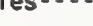 & \\
\hline$x$ & $Y$ & 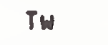 & If & $w$ & $P$ & v & RE & R & $h$ & MU & $1 \mathrm{~m}$ & WeW & Wtf & Wre & Wh & Wnu \\
\hline $\mathrm{cm}$ & $\mathrm{cn}$ & $k$ & K & $K$ & $\mathrm{kPa}$ & $\mathrm{m} / \mathrm{s}$ & & & 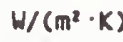 & & & K & $k$ & $x$ & $\%$ & $\%$ \\
\hline .000 & 5 & 2.73 & 99.03 & 9.10 & .6 & 0 & 6669 & .661 & 8856 & 53.32 & 6.55 & 10 & 0.99 & 1.27 & 2.10 & . \\
\hline 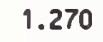 & 8 & 9.50 & 10 & 1.87 & 7 & & .67 & .662 & 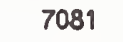 & & 55 & & 1.46 & 27 & & $\cdot$ \\
\hline 527 & 0 & 6.89 & 4.54 & 2 & 9.7 & 7 & 3890 & .662 & 5 & 37 & 7.31 & & 1.89 & 27 & 22 & 7.4 \\
\hline 81 & 18 & 6 & 6.69 & .77 & 8 & .10 & 3552 & .662 & 2 & 88 & 6.65 & & 2.49 & 87 & & .88 \\
\hline 5.080 & 0.318 & 13.28 & 8.84 & 93 & .8 & .23 & 3232 & .663 & 771 & 1.40 & 4.47 & 0 & 3.16 & 7 & & 3.37 \\
\hline 6.363 & 0.318 & 37 & 61.37 & 7 & .9 & .40 & 12921 & 0.663 & 786 & 0.75 & 3.68 & & 3.87 & 27 & & .00 \\
\hline 7.68 & 0.330 & .47 & 3.68 & 9 & .0 & .55 & 12632 & 0.663 & 708 & .67 & 2.44 & & 4.57 & 7 & & .70 \\
\hline 8.903 & -0.305 & 2.88 & 86.23 & 4 & .0 & .72 & 12352 & 0.663 & 5622 & 8.58 & 1.20 & & 5.13 & & & .25 \\
\hline .160 & -0.318 & 62.02 & 98.43 & 398.55 & 6948.1 & .86 & 12094 & 0.663 & 5805 & 28.90 & 1.35 & & 5.71 & & & 1.28 \\
\hline 1.430 & -0.318 & 72.04 & 410.46 & 10.59 & 6946.2 & .99 & 11852 & 0.664 & 18 & 28.39 & 0.66 & & 6.31 & & &. .37 \\
\hline .700 & -0.305 & 82.65 & 422.33 & 22.47 & 6944.2 & .10 & 11623 & 0.664 & 6059 & 29.00 & 1.21 & & 6.93 & & & 3.42 \\
\hline .970 & -0.318 & 92.82 & 435.03 & 35.17 & 2.3 & .28 & 11391 & 0.664 & 6968 & 32.69 & 5.01 & & 7.63 & & & 4.82 \\
\hline .240 & -0.953 & 83.54 & 447.43 & 47.58 & 6940.3 &. .44 & 11174 & 0.664 & 7748 & 35.66 & 37.22 & & 8.26 & & & 6.88 \\
\hline 2.527 & -2.223 & 93.63 & 26.71 & 26.78 & 6959.7 & 28.74 & 12750 & 0.662 & 5528 & 31.41 & 34.80 & & 1.89 & & & 7.36 \\
\hline 5.080 & -2.235 & 29.41 & 53.06 & 53.14 & .8 & .00 & 12 & 0.663 & 4870 & & 29.27 & & 3.16 & & & 7.90 \\
\hline 7.607 & -2.235 & 51.22 & 379.87 & 379.97 & 6952.0 & .31 & 11 & 0.663 & 5262 & 27.05 & 9.74 & & 4.57 & & & .28 \\
\hline .173 & -2.223 & 474.54 & 406.99 & 407.10 & 6948.1 & 35.64 & 10991 & 0.663 & 5462 & 26.81 & 29.17 & & 5.72 & & & 10.86 \\
\hline 2.700 & -2.223 & 95.69 & 432.78 & 32.90 & & & & 0.664 & & & & & 6.93 & & & 13.00 \\
\hline 2.540 & 2.197 & & 326.54 & 26.61 & & & & 0.662 & & & & & 1.89 & & & 7.37 \\
\hline 5.080 & 2.223 & 22.10 & 352.46 & 352.55 & & & & 0.663 & & & & & 316 & & & 3.14 \\
\hline 7.620 & & & 379.11 & & & & & & & & & & 4.57 & & & 9.34 \\
\hline 0.147 & 2.223 & & 405.53 & & & & & & & & & & 5.71 & & & 0.92 \\
\hline 2.713 & 2.223 & 493.80 & & & & & 10681 & & & & & & 6.94 & & & 3.09 \\
\hline 7.607 & -3.493 & 467.61 & 388.59 & 388.67 & & & & & & & & & 4.57 & & & 8.84 \\
\hline 7.633 & 3.493 & 467.88 & 388.92 & 389.00 & 6951.9 & 30.76 & 10232 & 0.663 & 4753 & 24.05 & 26.62 & 1.10 & 4.58 & 11.27 & 7.36 & 8.85 \\
\hline
\end{tabular}


Channel Specimen

Experiment 7

Date: 9 August 1990

Time: $14: 56: 11$

$\begin{array}{cccccccc}T A & T B & M & P O & P 0-P 1 & V f & a t & \text { Hqt } \\ K & K & \mathrm{~kg} / \mathrm{h} & \mathrm{KPa} & \mathrm{KPa} & \mathrm{X} & W & X \\ 299.45 & 412.15 & 31.35 & 6951.1 & 40.17 & 50.81 & 5092.0 & 1.20\end{array}$

Hot-side Temperatures:

$\begin{array}{ccc}X & Y & T H \\ \mathrm{~cm} & \mathrm{~cm} & K \\ 1.588 & 0.655 & 379.09 \\ 2.540 & 0.655 & 385.42 \\ 5.080 & 0.655 & 403.69 \\ 7.620 & 0.655 & 423.38 \\ 10.160 & 0.655 & 437.96 \\ 12.700 & 0.655 & 451.40 \\ 13.653 & 0.655 & 459.46\end{array}$

Insulated-Side Temperatures and Colculated Data:

\begin{tabular}{|c|c|c|c|c|c|c|c|c|c|c|c|c|c|c|c|c|}
\hline $\begin{array}{r}x \\
\mathrm{~cm}\end{array}$ & $\begin{array}{r}\mathrm{Y} \\
\mathrm{Cm}\end{array}$ & $\begin{array}{l}T H \\
K\end{array}$ & $\begin{array}{r}\text { Tf } \\
k\end{array}$ & $\begin{array}{c}\text { Taw } \\
K\end{array}$ & $\begin{array}{c}\mathrm{P} \\
\mathrm{kPa}\end{array}$ & $\begin{array}{c}v \\
\mathrm{~m} / \mathrm{s}\end{array}$ & E & PR & $\begin{array}{c}h \\
H /\left(m^{2} \cdot K\right)\end{array}$ & NU & NUm & $\begin{array}{l}\text { Hew } \\
K\end{array}$ & $\begin{array}{c}\text { Utf } \\
K\end{array}$ & $\begin{array}{c}\text { Ure } \\
x\end{array}$ & th & $\begin{array}{l}\text { Hnu } \\
\%\end{array}$ \\
\hline 0.000 & 0.965 & 23.05 & 29.29 & 99.43 & 6951.1 & 41.47 & 21205 & 0.662 & 12609 & $\pi 5.88$ & 79.14 & 1.10 & 0.78 & 1.27 & 12.64 & 13.57 \\
\hline 1.270 & 0.318 & 51.15 & 08.11 & 08.26 & 947.7 & 2.67 & 20800 & 0.662 & 9511 & 56.16 & 60.35 & .10 & 1.07 & 1.27 & 5.42 & 7.32 \\
\hline 2.527 & 0.330 & 63.15 & 16.91 & 17.07 & 944.4 & 3.87 & 0412 & 0.662 & 8017 & 46.48 & 50.10 & .10 & .36 & 1.27 & 5.82 & 7.62 \\
\hline 3.810 & 0.318 & 72.95 & 25.30 & 25.47 & 41.0 & 5.01 & 0059 & 0.662 & 7615 & 43.39 & 6.78 & .10 & .77 & 1.27 & 6.25 & 7.96 \\
\hline 5.080 & 0.318 & 81.62 & 33.69 & 33.87 & 937.7 & .96 & 9719 & 0.662 & $\pi 75$ & 43.57 & 6.91 & .10 & 2.23 & 1.27 & 6.79 & 8.39 \\
\hline 6.363 & 0.318 & 90.69 & 42.35 & 42.54 & 3 & 7.34 & 9383 & 0.663 & 7796 & 42.95 & 6.19 & .10 & 2.72 & 1.27 & 7.47 & 8.94 \\
\hline 7.620 & -0.330 & 99.78 & 50.86 & 51.05 & & & 9065 & .663 & & 41.69 & & & 5.21 & 1.27 & 8.20 & 9.56 \\
\hline 8.903 & -0.305 & 09.77 & 59.52 & 59.73 & & & $18 \pi 34$ & 0.663 & & 39.85 & 42.82 & & 3.60 & 11.27 & 8.68 & 9.98 \\
\hline .160 & -0.318 & 15.69 & & 68.17 & & & 18462 & 0.663 & & 40.70 & & & 4.01 & & 9.79 & 10.95 \\
\hline .430 & -0.318 & 22.89 & 376.26 & 76.49 & & & & 0.663 & & & & & 4.43 & & 10.81 & 11.88 \\
\hline .700 & -0.305 & 30.33 & 384.46 & 384.70 & & & & 0.663 & & & & & 4.86 & & 11.80 & 12.79 \\
\hline .970 & -0.318 & 38.35 & 393.23 & 393.48 & & & & 0.663 & & 44.96 & & & 5.35 & 11.27 & 12.85 & 13.76 \\
\hline .240 & .0 .953 & 30.53 & 401.80 & 402.05 & & & 17393 & 0.663 & & 48.42 & 50.30 & & 5.79 & 11.27 & 24.29 & 24.78 \\
\hline 2.527 & -2.223 & 67.92 & 18.68 & 318.82 & & & & 0.662 & & 43.45 & & & 1.36 & 11.27 & 5.67 & 7.51 \\
\hline 5.080 & -2.235 & & 337.13 & 337.28 & & & & 0.662 & & 34.67 & & & 2.23 & & 6.04 & 7.79 \\
\hline 7.607 & -2.235 & 409.60 & 355.89 & 356.06 & & & & 0.663 & & 37.58 & & & 3.20 & 11.27 & 7.67 & 9.11 \\
\hline 0.173 & -2.223 & 25.03 & & 375.05 & & & & 0.663 & & 38.21 & & & 4.01 & 11.27 & 9.41 & 10.62 \\
\hline 12.700 & -2.223 & 40.17 & 392.92 & 393.12 & & & & & & 38.93 & & & 4.86 & 11.27 & 1.50 & 12.51 \\
\hline 2.540 & 2.197 & 368.06 & & & & & 18868 & 0.662 & & 43.09 & & & 1.36 & 11.27 & 5.66 & 7.50 \\
\hline 5.080 & 2.223 & 388.47 & 336.42 & 336.58 & & & 18185 & 0.662 & & 39.87 & & & 2.23 & 11.27 & 6.48 & 8.14 \\
\hline 7.620 & 2.223 & 407.66 & 354.93 & 355.11 & & & 17541 & 0.663 & & 38.36 & & & 3.21 & 11.27 & 7.7 & 9.20 \\
\hline 10.147 & 2.223 & 423.98 & 373.28 & 373.47 & 6924.3 & 47.81 & 16954 & 0.663 & 7294 & 37.94 & 40.69 & 1.10 & 4.00 & 11.27 & 9.32 & 10.54 \\
\hline 12.713 & 2.223 & 439.25 & 391.27 & 391.48 & 6917.6 & 50.10 & 16421 & 0.663 & 7637 & 38.49 & 41.02 & 1.10 & 4.87 & 11.27 & 11.36 & 12.38 \\
\hline 7.607 & -3.493 & 425.51 & 364.10 & 364.23 & 6931.0 & 39.99 & 14790 & 0.663 & 6116 & 32.34 & 35.23 & 1.10 & 3.20 & 11.27 & 7.02 & 8.57 \\
\hline 7.633 & 3.493 & 423.16 & 363.01 & 363.15 & 6931.0 & 40.69 & 15122 & 0.663 & 6246 & 33.09 & 36.00 & 1.10 & 3.21 & 11.27 & 7.12 & 8.66 \\
\hline
\end{tabular}


Channel Specimen

Experiment 7

Oate: 9 August 1990

Time: 15:01:22

$\begin{array}{cccccccc}\text { TA } & T B & M & P O & P 0-P 1 & V f & \text { ot } & \text { Hqt } \\ K & K & \mathrm{~kg} / \mathrm{h} & \mathrm{KPa} & \mathrm{KPB} & \% & W & \% \\ 298.73 & 385.01 & 40.86 & 6972.9 & 61.06 & 50.85 & 5080.0 & 1.31\end{array}$

Hot-side Temperatures:

$\begin{array}{ccc}X & Y & T W \\ \mathrm{~cm} & \mathrm{~cm} & K \\ 1.588 & 0.655 & 366.17 \\ 2.540 & 0.655 & 370.55 \\ 5.080 & 0.655 & 384.48 \\ 7.620 & 0.655 & 399.61 \\ 10.160 & 0.655 & 410.57 \\ 12.700 & 0.655 & 420.71 \\ 13.653 & 0.655 & 427.46\end{array}$

Insulated-Side Temperatures and Calculated Data:

\begin{tabular}{|c|c|c|c|c|c|c|c|c|c|c|c|c|c|c|c|c|}
\hline$x$ & $Y$ & $T_{H}$ & Tf & $a w$ & P & v & RE & $R$ & h & MU & $\mathbf{w}$ & New & Wef & Wre & Wh & Unu \\
\hline $\mathrm{cm}$ & (iv & $\mathbf{K}$ & K & $k$ & $\mathrm{kPo}$ & $\mathrm{m} / \mathrm{s}$ & & & $H /\left(m^{2} \cdot k\right)$ & & & a & K & $x$ & $x$ & $x$ \\
\hline 0.000 & 0.965 & 16.89 & 98.45 & 98.70 & 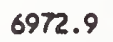 & .24 & 7946 & .661 & 25 & 0 & 101.70 & r & 68 & 1.27 & . & 4.22 \\
\hline 1.270 & .318 & 39.79 & 35.14 & 05.40 & 7.8 & .45 & 7540 & .662 & & & & & .87 & & & 7.60 \\
\hline 2.527 & .330 & 8.89 & & 12.08 & & & 146 & .662 & & & & & .07 & & . & 7.84 \\
\hline .810 & .318 & 1 & 7 & 18.45 & & & 83 & .662 & & & 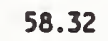 & & .38 & & & 8.11 \\
\hline 5.080 & 8 & 63.07 & 24.53 & 24.82 & $\cdots$ & $m$ & 30 & .662 & 7001 & 5 & 8.77 & 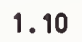 & .72 & 27 & 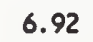 & 8.49 \\
\hline .363 & 8 & 7 & 8 & 39 & & & 78 & .662 & 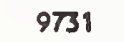 & 1 & .25 & 1.10 & .09 & 7 & 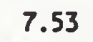 & .00 \\
\hline .6 & 0 & 1 & 3 & 5 & & & 3 & 62 & 9 & 6 & 7 & 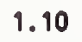 & .47 & 7 & & 55 \\
\hline 9 & .305 & 8 & 0 & 3 & & & & 63 & 9 & & 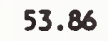 & 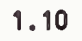 & 2.77 & 7 & & .87 \\
\hline .1 & .318 & 96 & 9 & 33 & & & 9 & 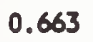 & & & 34 & 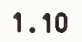 & 3.08 & 7 & 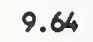 & .83 \\
\hline .430 & .318 & 4.57 & 8 & 57.14 & & & 00 & 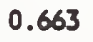 & 200 & & 6 & 1. & 3.40 & 7 & 6 & .65 \\
\hline .700 & .305 & 00.15 & 9 & 63.36 & & 24 & 12 & 63 & 383 & 7 & .2 & 1.9 & .73 & 7 & 9 & 2.50 \\
\hline .970 & .318 & 06.79 & 84 & 70.02 & & & 214 & 663 & & & 60.11 & & .11 & & & 3.23 \\
\hline •c & 3 & 8 & 3 & 2 & & & 30 & 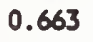 & & & .40 & & 5 & & & 81 \\
\hline 2.5 & ? & 52.45 & & & & & 90 & 2 & & & 9.03 & & .07 & & & \\
\hline 5.080 & .2 & 55 & 4 & 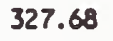 & & & 82 & $=$ & & & & & .72 & & & \\
\hline 7.60 & -2.2 & 29 & 30 & 16 & & & 09 & 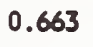 & 0 & & & & 6 & & & 6 \\
\hline - 1 & -2.2 & 26 & & 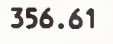 & & & & 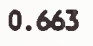 & & & & & 8 & & & \\
\hline 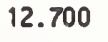 & -2.2 & 3 & 3 & & & & & 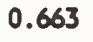 & & & & & .13 & & & \\
\hline 2.5 & & & & & & & & & & & & & 8 & & & \\
\hline 5.080 & 2 & 0 & & & & & & & & & & & 1.72 & & & \\
\hline 7.620 & 3 & 383.30 & 340.83 & & & & & 05 & & & & & 2.47 & & & 8 \\
\hline .147 & 2.223 & 395.84 & 354.80 & & & & & 0.063 & & & & & 3.07 & & & 10.39 \\
\hline 12.713 & 2.223 & 407.50 & 368.49 & 368.81 & & 6 & 22403 & 0.663 & 06 & & & 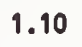 & 3.73 & .27 & 11.02 & 12.07 \\
\hline 7.607 & -3.493 & 400.25 & 349.36 & 349.56 & 6942.4 & 48.77 & 19334 & 0.663 & & & & 0 & 2.46 & 11.27 & 6.88 & 8.46 \\
\hline .633 & 3.493 & 396.87 & 347.76 & 347.97 & 6942.3 & 50.29 & 20086 & 0.663 & 7645 & 41.68 & 44.82 & 1.10 & 2.47 & 11.27 & 7.04 & 8.59 \\
\hline
\end{tabular}


Channel Specimen

Experiment 8

Date: 13 August 1990

Time: $15: 46: 12$

$\begin{array}{cccccccc}\text { TA } & T B & M & P O & P 0-P 1 & V f & \text { Qt } & \text { Jqt } \\ K & K & \mathrm{~kg} / \mathrm{h} & \mathrm{KPa} & \mathrm{KPa} & \times & W & \times \\ 296.57 & 676.53 & 13.84 & 6984.0 & 13.66 & 76.15 & 7579.0 & 1.04\end{array}$

Hot-side Temperatures:

$\begin{array}{ccc}X & Y & T H \\ \mathrm{~cm} & \mathrm{~cm} & K \\ 1.588 & 0.655 & 513.79 \\ 2.540 & 0.655 & 542.00 \\ 5.080 & 0.655 & 607.21 \\ 7.620 & 0.655 & 677.95 \\ 10.160 & 0.655 & 737.99 \\ 12.700 & 0.655 & 762.47 \\ 13.653 & 0.655 & 784.03\end{array}$

Insulated-Side Temperatures and Calculated Data:

\begin{tabular}{|c|c|c|c|c|c|c|c|c|c|c|c|c|c|c|c|c|}
\hline $\begin{array}{c}x \\
\mathrm{~cm}\end{array}$ & $\begin{array}{c}Y \\
\mathrm{~cm}\end{array}$ & TH & $\begin{array}{r}\text { Tf } \\
k\end{array}$ & $\begin{array}{c}\text { Taw } \\
K\end{array}$ & $\underset{K P O}{P}$ & $\begin{array}{c}\mathrm{V} \\
\mathrm{m} / \mathrm{s}\end{array}$ & 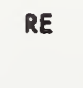 & PR & $\begin{array}{c}h \\
W /\left(m^{2} \cdot k\right)\end{array}$ & NU & m & $\begin{array}{c}\text { Htw } \\
K\end{array}$ & $\begin{array}{l}\text { Wtf } \\
k\end{array}$ & $\begin{array}{c}\text { Wre } \\
\%\end{array}$ & $\begin{array}{l}\text { Wh } \\
\%\end{array}$ & $\begin{array}{l}\text { Wnu } \\
x\end{array}$ \\
\hline 0.000 & -0.965 & 77.50 & 296.54 & 296.56 & 6984.0 & 17.65 & 9201 & 0.661 & 5475 & 33.13 & 37.83 & 1.10 & 2.06 & 11.27 & 11.63 & 12.63 \\
\hline 1.270 & -0.318 & 459.61 & 326.99 & 327.02 & 6982.8 & 19.40 & 8622 & 0.662 & 4579 & 26.00 & 31.35 & 1.10 & 3.30 & 11.27 & 4.80 & 6.88 \\
\hline 2.527 & -0.330 & 504.26 & 357.39 & 357.42 & 6981.7 & 21.15 & 8122 & 0.663 & 3744 & 20.05 & 24.23 & 1.10 & 4.36 & 11.27 & 5.34 & 7.26 \\
\hline 3.810 & -0.318 & 537.86 & 386.36 & 386.40 & 6980.6 & 22.82 & 7704 & 0.663 & 3553 & 18.05 & 21.65 & 1.10 & 5.82 & 11.27 & 5.90 & 7.68 \\
\hline 5.080 & -0.318 & 568.43 & 415.33 & 415.38 & 6979.4 & 24.49 & 7335 & 0.664 & 3610 & 17.47 & 20.76 & 1.18 & 7.42 & 11.27 & 6.54 & 8.19 \\
\hline 6.363 & -0.318 & 600.29 & 445.22 & 445.28 & 6978.3 & 26.21 & 6995 & 0.664 & 3604 & 16.64 & 19.61 & 1.31 & 9.10 & 11.27 & 7.31 & 8.81 \\
\hline 7.620 & -0.330 & 633.84 & 474.59 & 474.66 & 6977.1 & 27.91 & 6695 & 0.664 & 3504 & 15.49 & 18.16 & 1.44 & 10.77 & 11.27 & 8.06 & 9.44 \\
\hline 8.903 & -0.305 & 668.24 & 504.51 & 504.59 & 6976.0 & 29.63 & 6419 & 0.664 & 3401 & 14.42 & 16.83 & 1.58 & 12.08 & 11.27 & 8.60 & 9.90 \\
\hline 10.160 & -0.318 & 694.02 & 533.62 & 533.70 & 6974.9 & 31.31 & 6176 & 0.664 & 3419 & 13.95 & 16.12 & 1.68 & 13.46 & 11.27 & 9.51 & 10.71 \\
\hline 1.430 & -0.318 & 712.80 & 562.31 & 562.40 & 6973.7 & 32.97 & 5956 & 0.664 & 3537 & 13.92 & 15.86 & 1.76 & 14.87 & 11.27 & 10.91 & 11.97 \\
\hline 2.700 & -0.305 & 731.82 & 590.63 & 590.73 & 6972.6 & 34.60 & 5757 & 0.665 & 3845 & 14.63 & 16.46 & 1.84 & 16.34 & 11.27 & 12.46 & 13.40 \\
\hline 3.970 & -0.318 & 751.17 & 620.91 & 621.02 & 6971.5 & 36.35 & 5560 & 0.665 & 4592 & 16.88 & 18.74 & 1.91 & 17.98 & 11.27 & 14.47 & 15.29 \\
\hline 15.240 & -0.953 & 732.40 & 650.48 & 650.60 & 6970.3 & 38.06 & 5383 & 0.665 & 5068 & 18.04 & 19.26 & 1.84 & 19.47 & 11.27 & 27.06 & 27.51 \\
\hline 5.080 & -2.235 & 593.98 & 423.13 & 423.18 & 6979.4 & 23.40 & 6796 & 0.664 & 3235 & 15.46 & 18.63 & 1.28 & 7.42 & 11.27 & 6.17 & 7.90 \\
\hline 7.607 & -2.235 & 655.67 & 485.96 & 486.02 & 6977.2 & 26.80 & 6182 & 0.664 & 3288 & 14.31 & 16.87 & 1.53 & 10.75 & 11.27 & 7.70 & 9.14 \\
\hline 10.173 & -2.223 & 718.38 & 549.51 & 549.58 & 6974.9 & 30.24 & 5679 & 0.664 & 3247 & 12.98 & 15.04 & 1.78 & 13.47 & 11.27 & 9.15 & 10.39 \\
\hline 12.700 & -2.223 & 753.05 & 609.94 & 610.03 & 6972.6 & 33.51 & 5283 & 0.665 & 3793 & 14.12 & 15.86 & 1.92 & 16.34 & 11.27 & 12.32 & 13.27 \\
\hline 2.540 & 2.197 & 517.19 & 363.06 & 363.09 & 6981.7 & 19.75 & 7387 & 0.663 & 3565 & 18.89 & 22.95 & 1.10 & 4.38 & 11.27 & 5.27 & 7.21 \\
\hline 5.080 & 2.223 & 585.97 & 425.77 & 425.81 & 6979.4 & 23.07 & 6630 & 0.664 & 3450 & 16.42 & 19.57 & 1.25 & 7.42 & 11.27 & 6.38 & 8.06 \\
\hline 7.620 & 2.223 & 663.44 & 490.23 & 490.29 & 6977.1 & 26.48 & 6019 & 0.664 & 3221 & 13.93 & 16.45 & 1.56 & 10.77 & 11.27 & 7.60 & 9.06 \\
\hline 10.147 & 2.223 & 717.93 & 554.12 & 554.20 & 6974.9 & 29.87 & 5531 & 0.664 & 3349 & 13.32 & 15.36 & 1.78 & 13.44 & 11.27 & 9.36 & 10.57 \\
\hline 12.713 & 2.223 & 751.76 & 616.78 & 616.87 & 6972.6 & 33.19 & 5136 & 0.665 & 4025 & 14.86 & 16.57 & 1.91 & 16.35 & 11.27 & 12.98 & 13.89 \\
\hline 7.607 & -3.493 & 670.86 & 493.97 & 494.03 & 6977.2 & 26.13 & 5864 & 0.664 & 3154 & 13.57 & 16.06 & 1.59 & 10.75 & 11.27 & 7.49 & 8.96 \\
\hline 7.633 & 3.493 & 701.26 & 510.41 & 510.46 & 6977.1 & 24.99 & 5311 & 0.664 & 2923 & 12.30 & 14.65 & 1.71 & 10.78 & 11.27 & 7.15 & 8.68 \\
\hline
\end{tabular}


Table 3 (continued)

Channel specimen

Experiment 8

Date: 13 August 1990

Time: $15: 53: 27$

$\begin{array}{cccccccc}\text { TA } & T B & M & P O & P 0-P 1 & V f & \text { Qt } & \text { Hqt } \\ K & K & \mathrm{~kg} / \mathrm{h} & \mathrm{KPa} & \mathrm{KPa} & \mathrm{X} & \mathbf{H} & \mathbf{X} \\ 296.56 & 585.58 & 18.46 & 6984.7 & 20.05 & 76.24 & 7690.0 & 1.05\end{array}$

Hot-side Temperatures:

$\begin{array}{ccc}X & Y & T H \\ \mathrm{~cm} & \mathrm{~cm} & K \\ 1.588 & 0.655 & 470.10 \\ 2.540 & 0.655 & 489.31 \\ 5.080 & 0.655 & 536.85 \\ 7.620 & 0.655 & 588.64 \\ 10.160 & 0.655 & 625.27 \\ 12.700 & 0.655 & 657.67 \\ 13.653 & 0.655 & 676.21\end{array}$

Insulated-Side Temperatures and Calculated DEta:

\begin{tabular}{|c|c|c|c|c|c|c|c|c|c|c|c|c|c|c|c|c|}
\hline$x$ & $Y$ & TH & Tf & 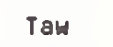 & 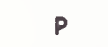 & $v$ & 5 & $R$ & $n$ & NU & & $t w$ & $f$ & Jre & th & Anu \\
\hline $\mathrm{cm}$ & $\mathrm{cm}$ & 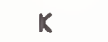 & K & $\mathbf{K}$ & $\mathrm{KPa}$ & $\mathrm{m} / \mathrm{s}$ & & & $H /\left(m^{2}\right.$ & & & $K$ & $K$ & $x$ & $x$ & x \\
\hline 000 & 5 & 6.84 & 6.51 & 6.55 & 7 & 0 & 52 & . & 7458 & 13 & . & & & & & 2.71 \\
\hline & & 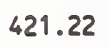 & & 8 & & & & & 6060 & & & & & & & 91 \\
\hline .527 & & 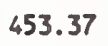 & 9 & 6 & 781 & & 20 & 63 & 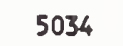 & & 44 & & 33 & & & 7.31 \\
\hline 810 & & . & 4.39 & 6 & & 4 & 761 & 663 & 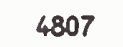 & 1 & 0 & & 3 & 1.27 & & 7.74 \\
\hline .080 & & 0. & 86.29 & 6 & 3.1 & .64 & 10344 & 663 & & 00 & 32 & & 5.65 & 27 & 6.65 & 8.27 \\
\hline 6.363 & & 3. & 08.87 & 18.96 & .4 & 39 & 52 & 54 & & 20 & 73 & & 5.93 & 7 & 7.47 & 8.94 \\
\hline 7.620 & & 7. & 31.07 & 1.17 & .7 & 11 & 9600 & .664 & 1 & .04 & 7 & & 3.19 & $? 7$ & 8.32 & 9.66 \\
\hline 8.903 & & 0.50 & 53.68 & 3.79 & .0 & 5.87 & 9270 & 0.664 & & 2.06 & 5.02 & & 9.19 & 1.27 & 9.03 & 0.28 \\
\hline 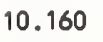 & & 36.54 & $\sqrt{5} .68$ & 5.80 & 1.4 & .58 & 8973 & 0.664 & & 2.18 & & & 0.24 & & & 1.40 \\
\hline 430 & & 3.05 & 97.36 & & & .26 & 8703 & 0.664 & & & & & 1.31 & & & 2.68 \\
\hline & & 1.98 & 18.76 & & & 92 & 8454 & 44 & & & & & 2.43 & & & 3.82 \\
\hline & & 0.34 & 41.65 & 0 & & 70 & 8205 & & & & & & 3.68 & & & 5.35 \\
\hline & & 3.8 & 64.00 & & & 44 & 79 & & & & & & 31 & & & 8.03 \\
\hline & & & 7 & & & 68 & $\infty 9$ & & & & & & 3.33 & & & 7.20 \\
\hline 5.080 & 2.235 & & 2.67 & 4 & & .07 & 52 & & & & & & 5.65 & & & 7.93 \\
\hline 7.607 & 2.235 & 4.69 & 0.39 & 8 & 7 & .52 & 33 & & & 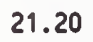 & 1 & & 8.18 & 7 & & 9.32 \\
\hline .173 & 2.223 & 07.22 & 88.66 & 38.77 & .4 & .02 & 8225 & at & & 35 & 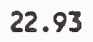 & & 0.25 & & & .92 \\
\hline 2.700 & -2.223 & 643.41 & 34.56 & 34.69 & 6968.0 & .35 & $\pi / 32$ & 0.664 & 63 & 64 & .85 & & 12.43 & & & 3.28 \\
\hline 2.540 & 2.197 & 64.77 & 46.66 & 46.72 & 69814 & 25.41 & 10256 & & & & .31 & & 3.34 & & & 7.23 \\
\hline 5.080 & & 16.06 & 93.94 & .01 & 6078 & 28.78 & 07 & & & & & & 5.65 & & & 8.07 \\
\hline 7.620 & 2.223 & 68.43 & 442.53 & 442.62 & & 32.25 & 8690 & & & & 3.94 & & 8.19 & & & 9.27 \\
\hline 0.147 & 2.223 & 604.85 & 490.70 & & & 35.70 & 8095 & & & & & & 10.23 & & & 11.18 \\
\hline 12.713 & 2.223 & 43.13 & 537.93 & 538.06 & & 39.08 & 7598 & & 243 & & 23.48 & 1.48 & 12.44 & & 12.72 & 13.64 \\
\hline 7.607 & -3.493 & 584.31 & 450.92 & & & & 8099 & & 4246 & 19.44 & 22.42 & 25 & 8.18 & & 7.54 & 9.01 \\
\hline 7.633 & 3.493 & 600.74 & 460.07 & 460.14 & 6974.7 & 29.97 & 7568 & 0.664 & 4026 & 18.18 & 21.05 & 1.31 & 8.20 & 11.27 & 7.30 & 8.80 \\
\hline
\end{tabular}


Channel Specimen

Experiment 8

Date: 13 August 1990

Time: $15: 59: 43$

$\begin{array}{cccccccc}\text { TA } & \text { TB } & \mathrm{M} & \mathrm{PO} & \mathrm{PO}-\mathrm{P1} & \mathrm{Vf} & \mathrm{Ot} & \text { Wqt } \\ \mathrm{K} & \mathrm{K} & \mathrm{kg} / \mathrm{h} & \mathrm{kPa} & \mathrm{kPa} & \mathrm{x} & \mathrm{H} & \mathrm{X}\end{array}$

$\begin{array}{llllllll}296.23 & 507.94 & 25.23 & 6985.1 & 30.76 & 76.28 & 7699.0 & 1.07\end{array}$

Hot-side Temperatures:

$\begin{array}{ccc}X & Y & T w \\ c m & c m & K \\ 1.588 & 0.655 & 435.27 \\ 2.540 & 0.655 & 448.08 \\ 5.080 & 0.655 & 482.55 \\ 7.620 & 0.655 & 519.60 \\ 10.160 & 0.655 & 546.40 \\ 12.700 & 0.655 & 571.06 \\ 13.653 & 0.655 & 585.94\end{array}$

Insulated-Side Temperatures and Calculated Data:

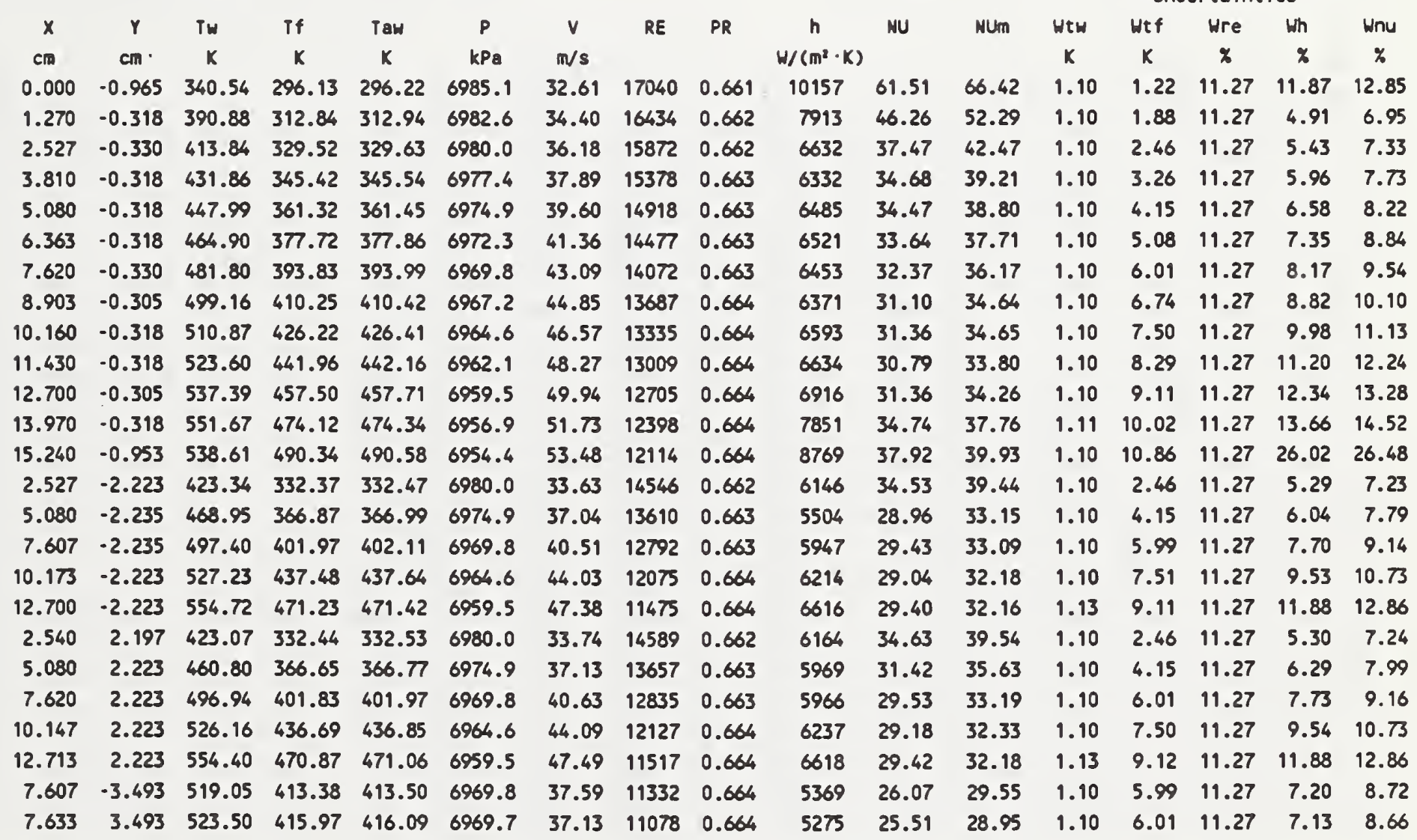


Channel Specimen

Experiment 8

Date: 13 August 1990

Time: 16:05:21

$\begin{array}{cccccccc}\text { TA } & T B & M & P O & P O-P 1 & V f & \text { Qt } & \text { Wqt } \\ K & K & \mathrm{~kg} / \mathrm{h} & \mathrm{KPa} & \mathrm{KPa} & \boldsymbol{X} & H & \mathrm{X} \\ 296.08 & 459.15 & 32.68 & 6987.0 & 44.67 & 76.33 & 7682.0 & 1.11\end{array}$

Hot-side Temperatures:

$\begin{array}{ccc}X & Y & T_{W} \\ \mathrm{~cm} & \mathrm{~cm} & K \\ 1.588 & 0.655 & 413.10 \\ 2.540 & 0.655 & 422.19 \\ 5.080 & 0.655 & 448.85 \\ 7.620 & 0.655 & 477.41 \\ 10.160 & 0.655 & 498.13 \\ 12.700 & 0.655 & 517.01 \\ 13.653 & 0.655 & 529.13\end{array}$

Insulated-Side Temperatures and Calculated Data:

\begin{tabular}{|c|c|c|c|c|c|c|c|c|c|c|c|c|c|c|c|c|}
\hline & & & & & & & & & & & & & & & 5 & \\
\hline$x$ & Y & Tw & Tf & Taw & $\mathbf{P}$ & $v$ & RE & PR & $n$ & NU & NUm & Wtw & Wtf & Wre & Wh & Wnu \\
\hline $\mathrm{cm}$ & $\mathrm{cm}$ & K & K & K & $\mathrm{kPa}$ & $\mathrm{m} / \mathrm{s}$ & & & $w /\left(m^{2} \cdot\right.$ & & & $k$ & K & $x$ & $x$ & $x$ \\
\hline 0.000 & .965 & 30.45 & 75.91 & 96.06 & 6987.0 & 2.61 & 22299 & 0.661 & 13060 & 79.13 & 84.08 & 1.10 & 0.99 & 1.27 & 2.07 & 3.04 \\
\hline 1.270 & -0.318 & 71.63 & 308.65 & 08.82 & 6983.3 & 4.40 & 21689 & 0.662 & 9796 & 57.78 & 3.99 & 1.10 & 1.48 & 1.27 & 9 & 7.01 \\
\hline 2.527 & -0.330 & 39.15 & 21.37 & 21.55 & 6979.6 & .19 & 21111 & 0.662 & 8244 & 47.35 & 2.61 & 1.10 & 1.91 & .27 & .47 & 7.36 \\
\hline 3.810 & -0.318 & 3.17 & 3.49 & 33.69 & 6975.8 & .91 & 20594 & 0.662 & 7849 & 43.99 & 8.83 & .10 & 2.52 & 7 & 5 & 7.72 \\
\hline 5.080 & -0.318 & 5.59 & 5.62 & 5.82 & 72. & .62 & 20106 & 0.663 & 8027 & .94 & .63 & 1 & 3.20 & 7 & 1 & 8.16 \\
\hline 6.363 & 8 & 28.60 & 2 & 8.34 & 6 & 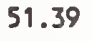 & 630 & 663 & 806 & 0 & 3 & 1.0 & 2 & 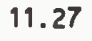 & & .73 \\
\hline 7.620 & 0 & 64 & 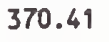 & 70.65 & 6 & 3 & 187 & 6 & 7 & 3 & ; & 10.6 & 3 & 7 & & .36 \\
\hline 8.903 & .0 .305 & 55.62 & 32.93 & 33.18 & 6 & 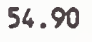 & 3760 & 0.663 & 778 & 39.82 & 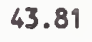 & 1.10 & 5.20 & & & .83 \\
\hline 60 & -0.318 & 64.26 & 25.11 & 75.38 & 6 & .63 & 18366 & 0.663 & 806 & .38 & 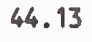 & 1.1 & 5.78 & 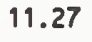 & 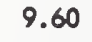 & .79 \\
\hline 30 & -0.318 & 74.29 & 407.11 & 7.40 & 6 & .34 & 996 & 0.663 & 805 & 4 & 1 & 1.1 & 6.39 & 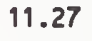 & 8 & .76 \\
\hline 00 & -0.305 & 84.98 & 418.96 & 19.26 & 6949 & .03 & 17648 & 0.664 & 836 & 40.27 & 3. & 1.10 & 7.02 & 7 & 9 & 2.68 \\
\hline 13.970 & -0.318 & 96.83 & 431.62 & 431.95 & 46. & .83 & 17293 & 0.664 & 933 & 44.03 & 7.57 & $\cdot 1$ & 7.72 & 7 & & 13.62 \\
\hline 15.240 & -0.953 & 85.36 & 443.99 & 44.33 & 6942. & .59 & 16962 & 0.664 & 024 & 47.38 & 6 & $\cdot 1$ & 8.37 & 7 & 6 & 24.66 \\
\hline 27 & -2.223 & 96.61 & 323.84 & 79 & 6 & 3 & 2 & 0.662 & 7674 & 5 & 2 & 1.10 & 1.91 & 7 & 4 & 7.26 \\
\hline 5.080 & -2.235 & 36.13 & 350.40 & 50.58 & 68 & 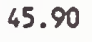 & 18183 & 0.6 & 6546 & 1 & 5 & 1.10 & 3.20 & 7 & 7 & 7.66 \\
\hline 7.607 & -2.235 & 55.42 & 377.43 & 377.64 & 6964.7 & .39 & 17293 & 0.663 & 726 & 37.52 & 41.60 & 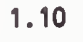 & 4.62 & 7 & 7 & 8.95 \\
\hline 10.173 & -2.223 & 477.65 & 404.77 & 405.01 & 6957.2 & 2.93 & 16491 & 0.663 & 7646 & 37.66 & 41.25 & 1.10 & 5.79 & 27 & 2 & 10.45 \\
\hline 12.700 & -2.223 & 499.26 & 430.77 & 431.03 & 6949.8 & 56.30 & 15806 & 0.664 & 8059 & 38.06 & 41.28 & 1.10 & 7.02 & 27 & 11.32 & 12.35 \\
\hline 2.540 & 2.197 & 396.86 & 323.71 & 323.86 & 6979.5 & 42.86 & 19358 & 0.662 & 7628 & 43.61 & 48.78 & .10 & 1.92 & 11.27 & .34 & 7.26 \\
\hline 5.080 & 2.223 & 426.21 & 349.89 & 350.07 & 6972.1 & 46.27 & 18372 & 0.663 & 7355 & 39.94 & 44.52 & .10 & 3.20 & 11.27 & .21 & 7.93 \\
\hline 7.620 & 2.223 & 454.06 & 376.81 & 377.02 & 6964.6 & 49.78 & 17475 & 0.663 & 7339 & 37.93 & 42.03 & 1.10 & 4.63 & 11.27 & 7.53 & 8.99 \\
\hline 10.147 & 2.223 & 477.06 & 403.48 & 403.72 & 6957.2 & 53.26 & 16683 & 0.663 & 7578 & 37.40 & 41.01 & .10 & 5.78 & 11.27 & .13 & 10.37 \\
\hline 12.713 & 2.223 & 499.01 & 429.64 & 429.91 & 6949.7 & 56.68 & 15983 & 0.664 & 7963 & 37.68 & 40.91 & 10 & 7.03 & 11.27 & 11.21 & 12.24 \\
\hline 307 & -3.493 & 477.53 & 388.79 & 388.95 & 6964.7 & 44.65 & 14885 & 0.663 & 6383 & 32.30 & 36.17 & 0 & 4.62 & 11 & .88 & 8.46 \\
\hline 7.633 & 3.493 & 477.56 & 388.99 & 389.16 & 6964.6 & 44.72 & 14897 & 0.663 & 6396 & 32.35 & 36.21 & 1.10 & 4.64 & 11.27 & 6.90 & 8.47 \\
\hline
\end{tabular}


Table 3 (continued)

Channel Specimen

Experiment 8

Date: 13 August 1990

Time: 16:11:03

$\begin{array}{cccccccc}T A & T 8 & M & P O & P O-P 1 & V f & \text { Qt } & \text { HqR } \\ K & K & \text { Kg/h } & \text { KPa } & \text { KPa } & \text { X } & \text { H } & \text { X } \\ 295.76 & 425.69 & 40.88 & 6991.9 & 63.11 & 76.23 & 7654.0 & 1.16\end{array}$

Hot-side Temperatures:

$\begin{array}{ccc}X & Y & T w \\ \mathrm{~cm} & \mathrm{~cm} & K \\ 1.588 & 0.655 & 397.39 \\ 2.540 & 0.655 & 403.94 \\ 5.080 & 0.655 & 425.32 \\ 7.620 & 0.655 & 448.20 \\ 10.160 & 0.655 & 464.66 \\ 12.700 & 0.655 & 479.60 \\ 13.653 & 0.655 & 489.94\end{array}$

Insulated-Side Temperatures and Calculated Data:

\begin{tabular}{|c|c|c|c|c|c|c|c|c|c|c|c|c|c|c|c|c|}
\hline $\begin{array}{c}x \\
c m\end{array}$ & $\mathrm{~cm}$ & $\begin{array}{l}T H \\
K\end{array}$ & k & $\begin{array}{l}\text { law } \\
K\end{array}$ & $\mathrm{KPa}$ & $\mathrm{m} / \mathrm{s}$ & $E$ & $P R$ & $H /\left(m^{2} \cdot k\right)$ & U & $\mathrm{m}$ & $\begin{array}{l}\text { Ktw } \\
\text { Nat }\end{array}$ & $\begin{array}{l}\text { Atf } \\
\mathrm{K}\end{array}$ & $x$ & $\%$ & Hnu \\
\hline 000 & .965 & 3.2 & 5. & D. & 6991.9 & 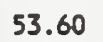 & 8133 & 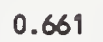 & & 8.18 & $0 \geq$ & & 0.85 & .27 & .35 & \\
\hline 270 & 318 & .98 & .56 & 32 & & & 23 & & & & & & 1 & & & \\
\hline 27 & & & & & & & 35 & 2 & & & & & & & 20 & \\
\hline 310 & & & & 48 & & & 02 & & & & & & 02 & 27 & 98 & \\
\hline 080 & & 2.97 & 4.76 & 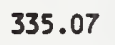 & & & 5893 & 0.662 & & & & & .56 & .27 & .49 & \\
\hline 363 & & & & & & & 92 & 3 & 30 & & & & & & & \\
\hline 620 & & 63 & 4.35 & 4.69 & & & 21 & 63 & 58 & 46 & & & 69 & & 34 & \\
\hline 903 & & & 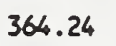 & . & & & & .005 & & & & & & & .29 & \\
\hline 160 & 18 & 1.71 & 3.86 & 4.24 & & & 35 & 0.663 & 333 & & & & .61 & 27 & .35 & 10. \\
\hline 430 & & & 334 & & & & & & & & & & & & 31 & 1. \\
\hline 700 & & & 2.70 & 2 & & & & 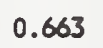 & & & & & & & 25 & 2. \\
\hline 970 & & 8.44 & 12.70 & 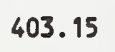 & & & 353 & 0.663 & & & & & .16 & & 2.04 & 3. \\
\hline 240 & .953 & 8.21 & 0 & 4 & & 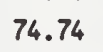 & 2483 & 0.664 & & & & & .67 & & 2.99 & 23. \\
\hline 527 & & & & & & & & & & & & & & & & \\
\hline .080 & & & 0. & & & & & 0.662 & & & & & & & 7 & \\
\hline .607 & .235 & 5.84 & 0.45 & 30 & & .09 & 07 & 0.663 & & 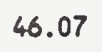 & & & .69 & & .32 & \\
\hline 173 & 23 & 2.63 & 23 & - & & & 39 & $4<3$ & & & & & .62 & & 04 & 0. \\
\hline .700 & 2.223 & 50.04 & 12.93 & & & & & & & & & & & 27 & 11.00 & 12. \\
\hline .540 & & & & & & & & & & & & & & & 5.41 & \\
\hline 080 & & & 7 & & & & & & & & & & & & & \\
\hline .620 & & & & & & & & & & & & & 3.69 & & .40 & \\
\hline 147 & & & & & & & & & & & & & 4.61 & 27 & .86 & (t) \\
\hline .713 & 2.223 & 460.06 & .43 & & & 6.80 & 21049 & 0.663 & & .63 & .26 & & .61 & .27 & 10.77 & 1. \\
\hline 307 & 93 & & & & & & & & & & & & 69 & 27 & 6.66 & \\
\hline & 3.493 & 5.11 & 0.30 & 0.54 & 60.3 & 12.91 & 9104 & .663 & 7553 & 39.49 & 43.70 & .10 & .70 & 1.27 & 0.74 & \\
\hline
\end{tabular}


Table 3 (continued)

Channel Specimen

Experiment 8

Date: 13 August 1990

Time: $16: 20: 46$

$\begin{array}{cccccccc}\text { TA } & T B & M & P O & P 0-P 1 & V f & \text { Ot } & \text { Wqt } \\ K & K & \mathrm{Kg} / \mathrm{h} & \mathrm{KPa} & \mathrm{KPa} & \% & W & \% \\ 295.73 & 582.37 & 18.38 & 7035.5 & 19.84 & 76.31 & 7596.0 & 1.05\end{array}$

Hot-side Temperatures:

$\begin{array}{ccc}X & Y & T H \\ \mathrm{~cm} & \mathrm{~cm} & K \\ 1.588 & 0.655 & 469.16 \\ 2.540 & 0.655 & 488.41 \\ 5.080 & 0.655 & 536.05 \\ 7.620 & 0.655 & 587.93 \\ 10.160 & 0.655 & 624.52 \\ 12.700 & 0.655 & 657.03 \\ 13.653 & 0.655 & 675.56\end{array}$

Insulated-Side Temperatures and Calculated Data:

\begin{tabular}{|c|c|c|c|c|c|c|c|c|c|c|c|c|c|c|c|c|}
\hline$x$ & $Y$ & TH & If & H & P & $v$ & RE & PR & II & IU & Jm & $t$ & tf & Ure & Wh & $u$ \\
\hline $\mathrm{cm}$ & $\mathrm{cm}$ & K & $K$ & K & $\mathrm{KPa}$ & $\mathrm{m} / \mathrm{s}$ & & & $\left(m^{2} \cdot k\right)$ & & & K & $K$ & \% & $x$ & $\%$ \\
\hline 0.000 & .965 & 5.88 & 95.68 & 5.73 & 7035.5 & 23.34 & 12311 & 0.661 & 7384 & 44.75 & 49.5 & 1.10 & 1.59 & 1.27 & 1.72 & 2.71 \\
\hline 1.270 & .318 & 0.37 & 8.53 & 8.58 & 033.8 & .09 & 11719 & 0.662 & 5978 & 4.53 & 40.22 & .10 & 2.51 & 1.27 & 4.84 & .90 \\
\hline 2.527 & 0.330 & 2.69 & 1.33 & 1.39 & 7032.2 & 83 & 11188 & 0.662 & 4951 & .32 & 1.91 & 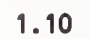 & 3.30 & 1.27 & 5.38 & 7.29 \\
\hline 3.810 & .318 & 7.22 & 33.07 & 63.14 & 030.5 & .49 & 10733 & 0.663 & 4727 & .04 & .10 & 1.10 & 4.40 & 11.27 & 5.95 & 7.72 \\
\hline 5.080 & .318 & .68 & 34.81 & 34.88 & 8.8 & .16 & 10318 & .063 & 824 & 30 & 38 & . 10 & 5.60 & .27 & 6.59 & .23 \\
\hline 6.363 & 0.318 & 3.01 & 07.23 & 07.32 & 327.2 & .88 & 9929 & 0.663 & 4840 & 3.74 & 7.24 & 1.10 & 6.87 & 1.27 & 7.38 & 8.87 \\
\hline 7.620 & .330 & 6.67 & 29.27 & 429.36 & 7025.5 & 56 & 9579 & 0.664 & 6766 & 22.56 & 5.77 & 10 & 8.12 & .27 & 8.20 & 9.56 \\
\hline 8.903 & 0.305 & 669.77 & 451.71 & 451.82 & 7023.9 & .28 & 9250 & 0.664 & 4729 & 21.62 & 24.56 & 1.19 & 9.12 & 11.27 & 8.90 & 10.17 \\
\hline .160 & .318 & 36.23 & 73.55 & 73.67 & 022.2 & .96 & 8956 & 0.664 & 4881 & 21.61 & .30 & 1. & 0.15 & .27 & .08 & 1.22 \\
\hline .430 & 0.318 & 2.72 & 5.08 & 0 & 020.6 & .61 & 8686 & 0.664 & 958 & 1.30 & 3.73 & 2 & 1.22 & .27 & 1.42 & 2.44 \\
\hline 2.700 & 0.305 & 621.64 & 516.32 & 516.46 & 7018.9 & 40.24 & 8439 & 0.664 & 169 & 21.58 & 23.90 & 1.39 & 12.33 & 11.27 & 12.60 & 13.53 \\
\hline 3.970 & 0.318 & 9.94 & 539.04 & 539.19 & 701 & 99 & 8191 & 0.664 & 5945 & 2 & 26.47 & 7 & 13.56 & .27. & 4.14 & 4.97 \\
\hline 5.240 & 0.953 & 24.93 & 61.22 & 61.39 & 7015.6 & 43.69 & 7966 & 0.664 & 38 & 25.7 & 27.34 & 1.41 & 14.69 & 11.27 & 26.46 & 26.92 \\
\hline 2.527 & 2.223 & .69 & 4.54 & 14.59 & 2.2 & 25.31 & 10392 & 0.6 & 50 & 24.96 & .46 & 1.10 & 3.30 & 11.27 & 5.24 & 7.19 \\
\hline 5.080 & 2.235 & 520.26 & 391.05 & 12 & 8 & 63 & 9539 & 0.663 & 88 & 21.61 & .28 & 10 & 5.60 & 11.27 & 6.19 & 7.90 \\
\hline 7.607 & -2.235 & 564.00 & 38.38 & 38.46 & 7025.6 & 32 & 8825 & 0.664 & 4453 & 20 & 23.87 & 1.16 & 8.11 & 27 & 7.81 & 9.23 \\
\hline .173 & -2.223 & 07.11 & 486.24 & 86.35 & 7022.2 & .45 & 8220 & 0.664 & 4548 & 19. & 22.35 & 1.34 & 0.16 & 11.27 & 9.54 & 10.74 \\
\hline 12.700 & -2.223 & 643.17 & 531.76 & 31.89 & 7018.9 & .71 & 7728 & 0.664 & 4886 & 19.99 & 22.19 & 1.48 & 12.33 & 11.27 & 12.00 & 12.97 \\
\hline 2.540 & 2.197 & 54.03 & 5.46 & 345.52 & .2 & 25.02 & 10230 & 0.663 & 4646 & 25 & .92 & 1.10 & 3.31 & 11.27 & 5.28 & 7.22 \\
\hline 5.080 & 2.223 & 515.47 & 392.39 & 392.45 & 7028.8 & 28.33 & 9386 & 0.663 & 4501 & 22.64 & 26.31 & 1.10 & 5.60 & 11.27 & 6.35 & 8.03 \\
\hline 7.620 & 2.223 & 567.97 & 440.62 & 440.70 & 7025.5 & 31.74 & 8673 & 0.664 & 4393 & 20.43 & 23.49 & 1.18 & 8.12 & 11.27 & 7.75 & 9.18 \\
\hline .147 & 2.223 & 604.12 & 488.43 & 488.53 & 022.3 & 1 & 8082 & 0.664 & 6754 & 20.61 & 23.17 & 1.32 & 10.14 & 1.27 & 9.86 & 11.02 \\
\hline 2.713 & 2.223 & & 35.31 & & 7018.9 & 38 & 7587 & 0.664 & 50 & 20 & 22.94 & 1.48 & 12.34 & 11.27 & 12.45 & 13.39 \\
\hline 7.607 & -3.493 & 583.17 & 448.58 & 448.66 & 7025.6 & 30.56 & 8108 & 0.664 & 156 & 19.09 & 22.05 & 1.24 & 8.11 & 11.27 & 7.46 & 8.93 \\
\hline 7.633 & 3.493 & 599.39 & 457.53 & 457.61 & 7025.5 & 29.54 & 7383 & 0.664 & 3943 & 17.87 & 20.73 & 1.30 & 8.13 & 11.27 & 7.22 & 8.74 \\
\hline
\end{tabular}


Channel specinen

Experiment 9

Date: 14 August 1990

Time: $14: 02: 28$

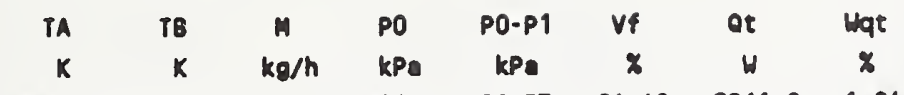

$\begin{array}{llllllll}296.79 & 654.80 & 17.94 & 7006.7 & 20.53 & 94.40 & 9261.0 & 1.04\end{array}$

Hot-side Temperatures:

$\begin{array}{ccc}X & Y & T W \\ \mathrm{Cm} & \mathrm{Cm} & K \\ 1.588 & 0.655 & 513.66 \\ 2.540 & 0.655 & 538.75 \\ 5.080 & 0.655 & 597.78 \\ 7.620 & 0.655 & 662.71 \\ 10.160 & 0.655 & 706.98 \\ 12.700 & 0.655 & 743.45 \\ 13.653 & 0.655 & 766.01\end{array}$

Insulated-Side Temperatures and Calculated Data:

\begin{tabular}{|c|c|c|c|c|c|c|c|c|c|c|c|c|c|c|c|c|}
\hline & & & & & & & & & & & & & & & & \\
\hline$x$ & $Y$ & $T_{H}$ & Tf & Тан & $\mathbf{P}$ & v & RE & PR & h & NU & NUm & Wtw & Wtf & Wre & Wh & Wnu \\
\hline $\mathrm{CNT}$ & $\mathrm{cm}$ & K & K & K & KPa & $\mathrm{m} / \mathrm{s}$ & & & $H /\left(m^{2} \cdot k\right)$ & & & K & K & $x$ & $x$ & $\%$ \\
\hline 0.000 & -0.965 & 71.99 & 96.73 & 96.78 & 006.7 & 23.02 & 12029 & 0.661 & 7199 & 43.54 & 9.30 & 1.10 & 1.95 & 11.27 & 11.66 & 2.65 \\
\hline 1.270 & 0.318 & 53.04 & 25.18 & 25.24 & 005.0 & .16 & 11319 & 0.662 & 5805 & 33.08 & 9.70 & 1.10 & 3.12 & 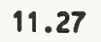 & 4.78 & 6.86 \\
\hline 2.527 & 0.330 & 93.98 & 53.58 & 53.64 & 7003.3 & .30 & 10700 & 0.663 & 787 & 25.81 & 1.02 & .10 & 4.11 & 7 & 5.33 & 7.25 \\
\hline 3.810 & 0.318 & 24.17 & 80.64 & 80.71 & 001.6 & .33 & 10180 & 0.663 & 583 & 23.52 & 28.05 & r & 5.48 & 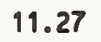 & 5.90 & 7.68 \\
\hline 5.080 & 0.318 & 51.68 & 17.70 & 07.78 & 6999.8 & .2 & 9716 & .66 & 4692 & .00 & 8 & 11 & 6.99 & & .55 & 8.19 \\
\hline 7.620 & 0.330 & 10.22 & 463.05 & 463.16 & 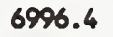 & 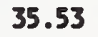 & 8906 & .664 & 4634 & 20. & 24.26 & .35 & 0.14 & 27 & 8.17 & 9.54 \\
\hline 8.903 & 0.305 & 40.54 & 91.00 & 91.12 & 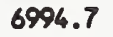 & .64 & 8555 & 0.664 & 4551 & 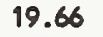 & .76 & .47 & 1.39 & 27 & 8.80 & 0.08 \\
\hline .160 & 0.318 & 61.29 & 19 & 18.32 & 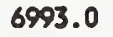 & 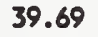 & 8243 & .664 & 4685 & 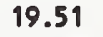 & .31 & .55 & 12.68 & 27 & 9.94 & 09 \\
\hline .430 & 0.318 & 80.71 & 44.98 & 45.13 & 69 & .71 & 7961 & 0.664 & 41 & 8 & 79 & .63 & 14.01 & 27 & 1.32 & 2.35 \\
\hline 700 & -0.305 & 703.72 & 71.43 & 71.59 & 6989.0 & .81 & 7704 & .665 & 0 & 3 & 21.90 & .72 & 15.39 & 27 & 2.53 & 13.46 \\
\hline 3.970 & -0.318 & 725.46 & 599.71 & 399.89 & 6987.9 & .84 & 7450 & 0.665 & 581 & 21.90 & 24.32 & 1.81 & 16.94 & .27 & 14.16 & 14.99 \\
\hline 15.240 & -0.953 & 707.29 & 627.34 & 627.53 & 6986.2 & 7.93 & 7221 & 0.665 & 6350 & 23.18 & 24.76 & 1.74 & 18.35 & 11.27 & 26.35 & 26.81 \\
\hline 5.080 & -2.235 & 76.67 & 415.39 & 415.46 & 6999.8 & 9.88 & 8972 & 0.664 & 4188 & 20.27 & 24.28 & 1.21 & 6.99 & .27 & 6.17 & 7.89 \\
\hline 7.607 & -2.235 & 631.65 & 474.27 & 474.37 & 6996.4 & 34.02 & 8194 & 0.664 & 4334 & 19.17 & 22.44 & 1.43 & 10.13 & 11.27 & 7.79 & 9.21 \\
\hline 10.173 & -2.223 & 87.59 & 533.83 & 533.95 & 6993.0 & 38.22 & 7553 & 0.664 & 4358 & 17.78 & 20.44 & 1.66 & 12.69 & 1.27 & 9.40 & 10.61 \\
\hline 12.700 & -2.223 & 728.98 & 590.46 & 590.61 & 6989 & 42.22 & 7044 & 0.665 & 4791 & 18.23 & 20.47 & 1.82 & 15.39 & .27 & 12.05 & 13.01 \\
\hline 2.540 & 2.197 & 08.84 & 359.52 & 359.57 & 7003 & 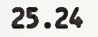 & 9627 & 0.663 & 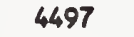 & 23.98 & 29.03 & 1.10 & 4.13 & 27 & 5.23 & 7.18 \\
\hline 5.080 & 2.223 & 72.16 & 418.70 & $418 . \pi 7$ & 69 & 9 & 8682 & 0.664 & 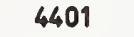 & 9 & 25.16 & 1.20 & 6.99 & 27 & 6.33 & 8.02 \\
\hline 7.620 & 2.223 & 641.25 & 479.53 & 479.63 & 6996 & .46 & 7912 & 0.664 & 42 & 18.52 & 21.73 & 1.47 & 10.14 & 27 & 7.65 & 9.10 \\
\hline 0.147 & 2.223 & 683.22 & 539.82 & 539.94 & 6993 & 37.59 & 7292 & 0.664 & 46 & 18.93 & 21.55 & 1.64 & 12.67 & 27 & 9.92 & 11.07 \\
\hline 12.713 & 2.223 & 728.19 & 598.95 & 599.10 & 6989.6 & 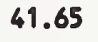 & 6785 & 0. & 5 & 1 & 57 & 32 & 15.41 & 27 & 12.81 & 13.72 \\
\hline 7.607 & -3.493 & 651.10 & 484.59 & 484.68 & 6996.4 & 32 & 7631 & 0.664 & 40 & 1 & & .51 & 0.13 & 27 & 7.50 & 8.97 \\
\hline 7.633 & 3.493 & 682.24 & 501.51 & 501.60 & 6996.4 & 31.26 & 6861 & 0.664 & 3773 & 16.07 & 19.03 & 1.64 & 10.16 & 11.27 & 7.13 & 8.66 \\
\hline
\end{tabular}


Table 4. Uncertainties in data analysis parameters and calculated quantities

\begin{tabular}{|c|c|c|c|}
\hline $\begin{array}{l}\text { Uncertainty } \\
\text { Parameter }\end{array}$ & $\begin{array}{l}\text { Major Source } \\
\text { of Uncertainty }\end{array}$ & $\begin{array}{l}\text { Magnitude of } \\
\text { Uncertainty }\end{array}$ & $\begin{array}{c}\text { Estimated or } \\
\text { Calculated }\end{array}$ \\
\hline $\begin{array}{l}\text { Channel Width } \\
\text { and Height }\end{array}$ & Measurement & $0.025 \mathrm{~mm}$ & Estimated \\
\hline $\begin{array}{l}\text { Length of } \\
\text { Heated Zone }\end{array}$ & Measurement & $1 \mathrm{~mm}$ & Estimated \\
\hline $\begin{array}{l}\text { Location of } \\
\text { Temperature Probe }\end{array}$ & Measurement & $1 \mathrm{~mm}$ & Estimated \\
\hline Channel Flow Rate & $\begin{array}{l}\text { Specimen Uni- } \\
\text { formity }\end{array}$ & 58 & Estimated \\
\hline Fluid Temperature & Channel Flow Rate & $0.5-21.0 \mathrm{~K}$ & Calculated \\
\hline Total Heat Flow & $\begin{array}{l}\text { Inlet and Outlet } \\
\text { Temperature }\end{array}$ & $1.0-2.08$ & Calculated \\
\hline Fluid Velocity & Channel Flow Rate & $6.9-7.58$ & Calculated \\
\hline Friction Factor & $\begin{array}{l}\text { Channel Height, } \\
\text { Pressure Taps }\end{array}$ & $\begin{array}{l}17-188 \text { for } \\
\operatorname{Re}>4000\end{array}$ & Calculated \\
\hline $\begin{array}{l}\text { Heat Transfer } \\
\text { Coefficient }\end{array}$ & Channel Flow Rate & $\begin{array}{l}5.9-12.98 \\
0.2<x / L<0.8\end{array}$ & Calculated \\
\hline Reynolds Number & $\begin{array}{l}\text { Viscosity Function, } \\
\text { Channel Flow Rate }\end{array}$ & 11.38 & Calculated \\
\hline Nusselt Number & Channel Flow Rate & $\begin{array}{l}7.6-13.88 \\
0.2<x / L<0.8\end{array}$ & Calculated \\
\hline
\end{tabular}


Table 5. Predicted flow distribution in heat transfer experiments using method of Appendix B

\begin{tabular}{|c|c|c|}
\hline Region & $\begin{array}{c}\text { Boundaries } \\
(\mathrm{y} / \mathrm{W})\end{array}$ & $\begin{array}{c}\text { Location of } \\
\text { Temp. Probe } \\
(\mathrm{y} / \mathrm{W})\end{array}$ \\
\hline \hline 1 & $-0.500,-0.364$ & 0.4442 \\
2 & $-0.364,-0.163$ & 0.2842 \\
3 & $-0.163,+0.120$ & 0.0420 \\
4 & $+0.120,+0.363$ & +0.2827 \\
5 & $+0.363,+0.500$ & +0.4442 \\
\hline
\end{tabular}

\begin{tabular}{|c|c|ccccc|}
\hline $\begin{array}{c}\text { Expt. } \\
\#\end{array}$ & $\begin{array}{c}\dot{\mathrm{m}} \\
(\mathrm{kg} / \mathrm{h})\end{array}$ & 1 & $\dot{\mathrm{m}}_{\mathrm{c}} \cdot \mathrm{n} / \dot{\mathrm{m}}$ for each region & \\
\hline \hline 3 & 4.06 & 0.9817 & 1.0034 & 1.0321 & 0.9958 & 0.9539 \\
3 & 8.41 & 0.9650 & 1.0055 & 1.0568 & 0.9917 & 0.9235 \\
3 & 14.94 & 0.9223 & 1.0021 & 1.0762 & 1.0022 & 0.9123 \\
3 & 20.00 & 0.8942 & 0.9992 & 1.0864 & 1.0112 & 0.9072 \\
3 & 29.39 & 0.8562 & 0.9971 & 1.1015 & 1.0217 & 0.8981 \\
3 & 39.79 & 0.8226 & 0.9946 & 1.1141 & 1.0316 & 0.8913 \\
\hline 4 & 10.16 & 0.9329 & 0.9973 & 1.0680 & 1.0031 & 0.9240 \\
4 & 14.90 & 0.9442 & 1.0054 & 1.0729 & 0.9950 & 0.9052 \\
4 & 20.34 & 0.9156 & 0.9996 & 1.0815 & 1.0067 & 0.9033 \\
4 & 30.30 & 0.8929 & 0.9966 & 1.0944 & 1.0142 & 0.8904 \\
4 & 40.04 & 0.8785 & 0.9951 & 1.1037 & 1.0183 & 0.8802 \\
\hline 5 & 13.33 & 0.9651 & 1.0072 & 1.0764 & 0.9851 & 0.8919 \\
5 & 17.31 & 0.9410 & 1.0090 & 1.0816 & 0.9930 & 0.8885 \\
5 & 23.81 & 0.9042 & 1.0040 & 1.0904 & 1.0073 & 0.8888 \\
5 & 31.08 & 0.8763 & 1.0034 & 1.1009 & 1.0144 & 0.8828 \\
5 & 40.64 & 0.8487 & 1.0027 & 1.1120 & 1.0215 & 0.8757 \\
\hline 6 & 3.78 & 0.9845 & 1.0051 & 1.0328 & 0.9937 & 0.9510 \\
6 & 7.08 & 0.9456 & 0.9974 & 1.0546 & 1.0021 & 0.9407 \\
6 & 13.61 & 0.9295 & 1.0042 & 1.0732 & 0.9997 & 0.9126 \\
6 & 19.90 & 0.8904 & 0.9998 & 1.0870 & 1.0122 & 0.9069 \\
6 & 28.62 & 0.8528 & 0.9970 & 1.1013 & 1.0229 & 0.8997 \\
\hline & 40.98 & 0.8104 & 0.9939 & 1.1178 & 1.0345 & 0.8922 \\
\hline
\end{tabular}


Table 5. (Continued)

\begin{tabular}{|c|c|cccccc|}
\hline $\begin{array}{c}\text { Expt. } \\
\#\end{array}$ & $\begin{array}{c}\dot{\mathrm{m}} \\
(\mathrm{kg} / \mathrm{h})\end{array}$ & 1 & $\begin{array}{c}\dot{\mathrm{m}}_{\mathrm{c}} \cdot \mathrm{n} / \dot{\mathrm{m}} \text { for each region } \\
2\end{array}$ & 3 & 4 & 5 \\
\hline \hline 7 & 9.27 & 0.9317 & 0.9866 & 1.0635 & 1.0104 & 0.9372 \\
7 & 15.90 & 0.9363 & 1.0023 & 1.0736 & 0.9998 & 0.9075 \\
7 & 21.95 & 0.9026 & 0.9998 & 1.0843 & 1.0110 & 0.9025 \\
7 & 31.35 & 0.8733 & 0.9994 & 1.0980 & 1.0181 & 0.8912 \\
7 & 40.86 & 0.8519 & 0.9990 & 1.1083 & 1.0233 & 0.8823 \\
\hline 8 & 13.84 & 0.9663 & 1.0071 & 1.0732 & 0.9866 & 0.8951 \\
8 & 18.46 & 0.9397 & 1.0084 & 1.0799 & 0.9953 & 0.8902 \\
8 & 25.23 & 0.9064 & 1.0039 & 1.0891 & 1.0070 & 0.8899 \\
8 & 32.68 & 0.8815 & 1.0037 & 1.0996 & 1.0131 & 0.8825 \\
8 & 40.88 & 0.8614 & 1.0034 & 1.1082 & 1.0185 & 0.8752 \\
8 & 18.38 & 0.9412 & 1.0084 & 1.0789 & 0.9945 & 0.8922 \\
\hline 9 & 17.94 & 0.9568 & 1.0123 & 1.0824 & 0.9849 & 0.8808 \\
\hline
\end{tabular}




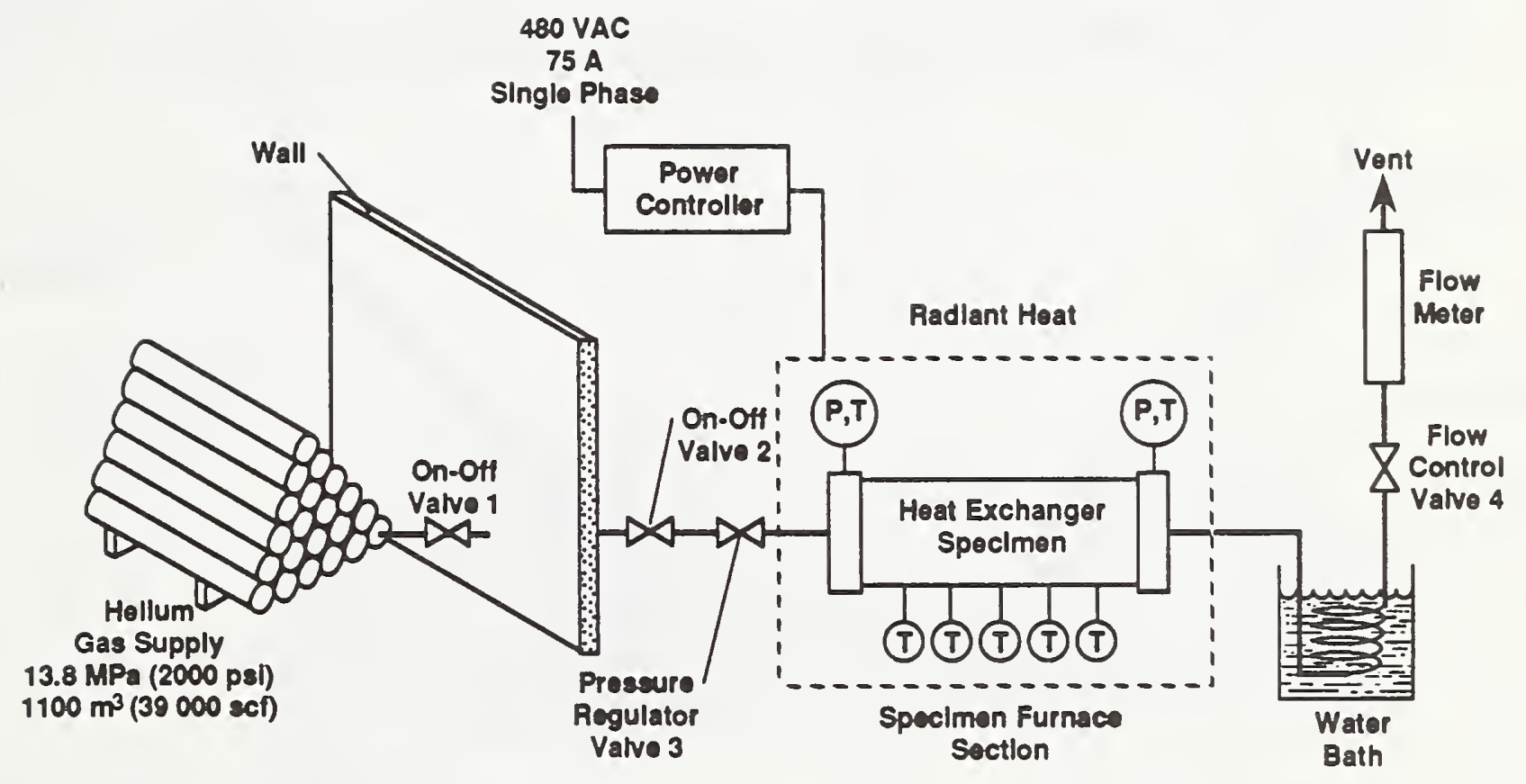

Figure 1. Helium flow apparatus. 


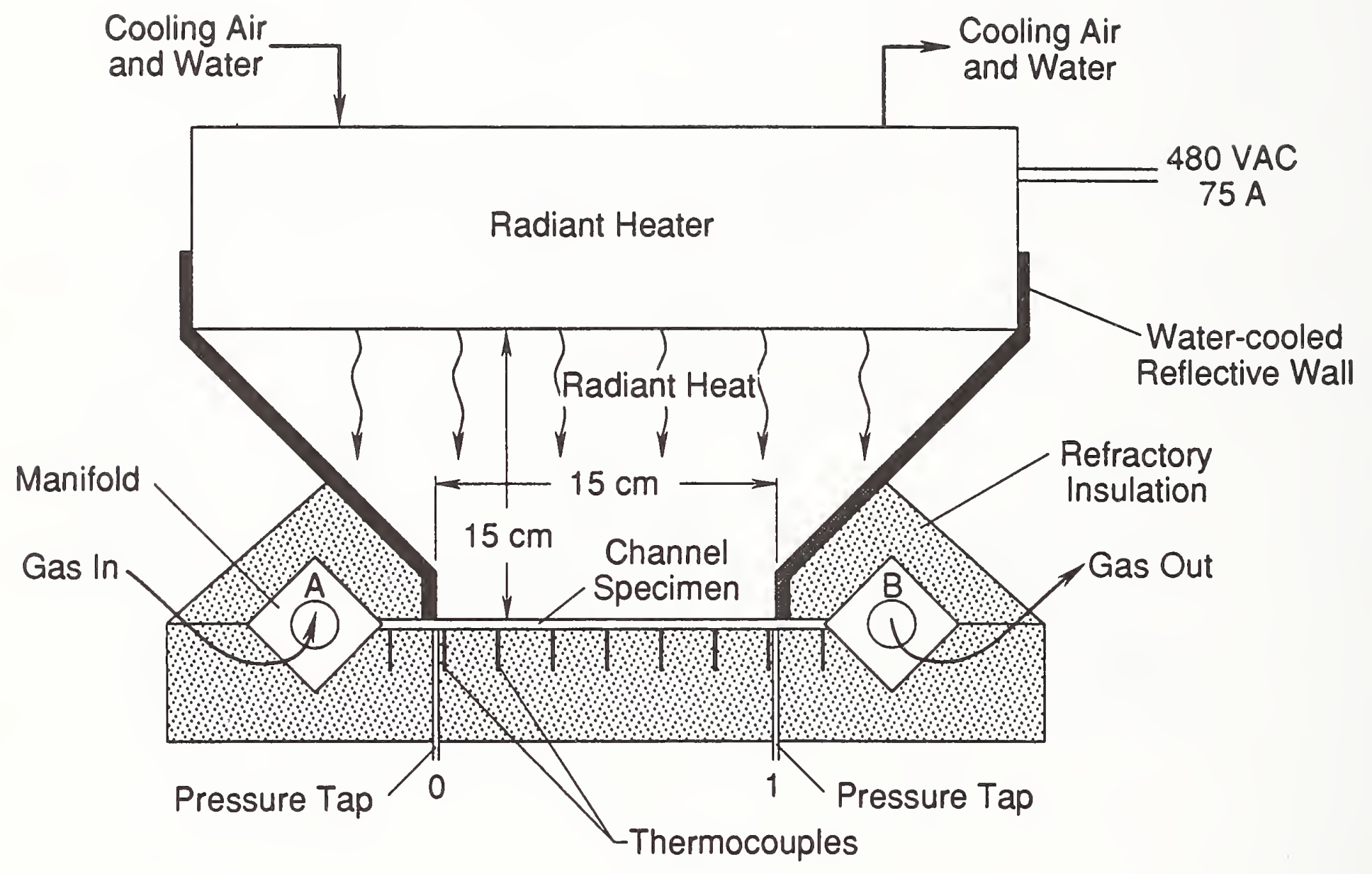

Figure 2. Specimen furnace, showing location of inlet gas temperature (A), upstream pressure (0), outlet gas temperature (B), and downstream pressure (1). 


\section{Channel Specimen \\ (end view)}

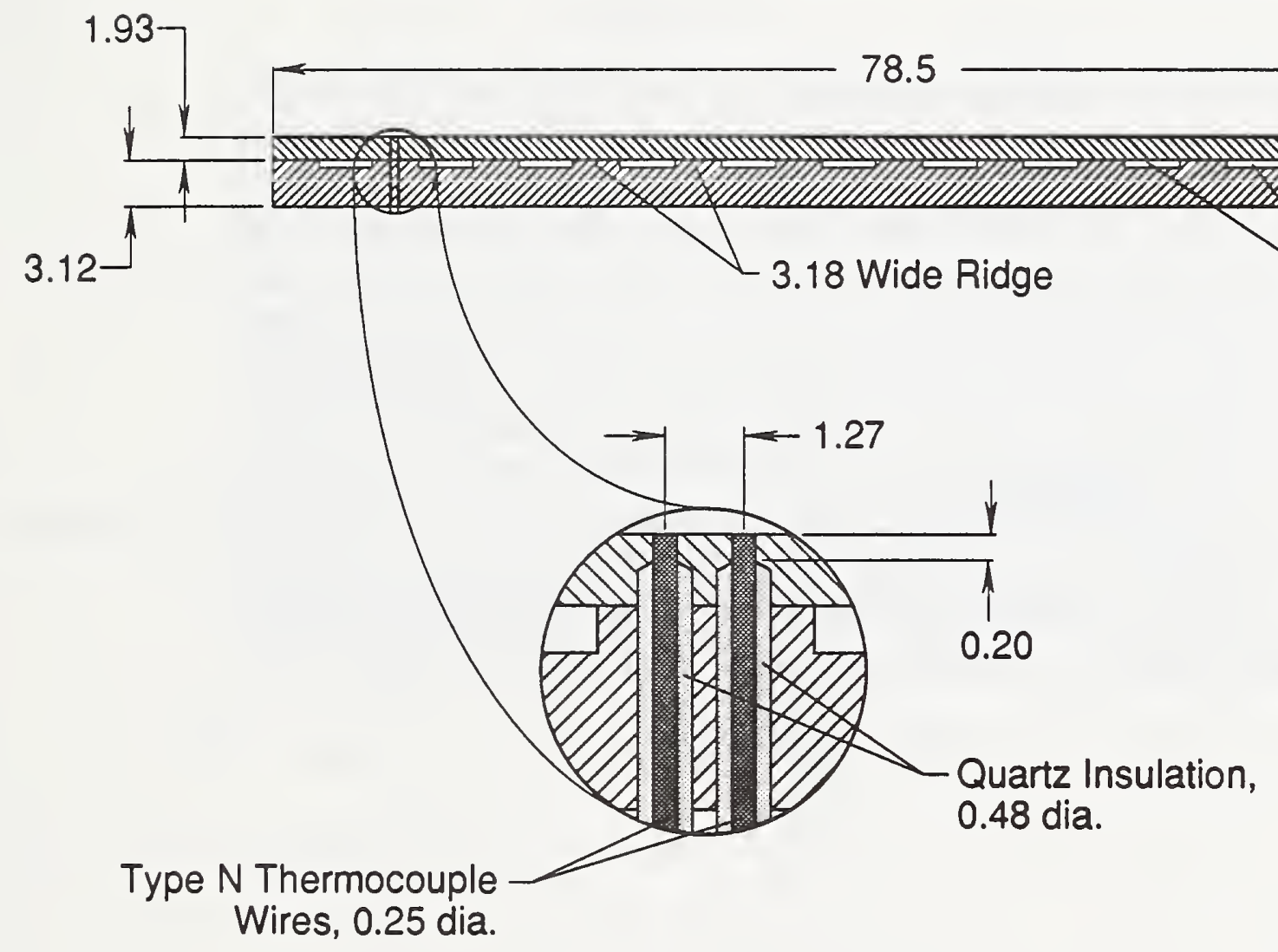

\section{NOTE:}

All dimesions in $\mathrm{mm}$.

Figure 3. Channel specimen. 


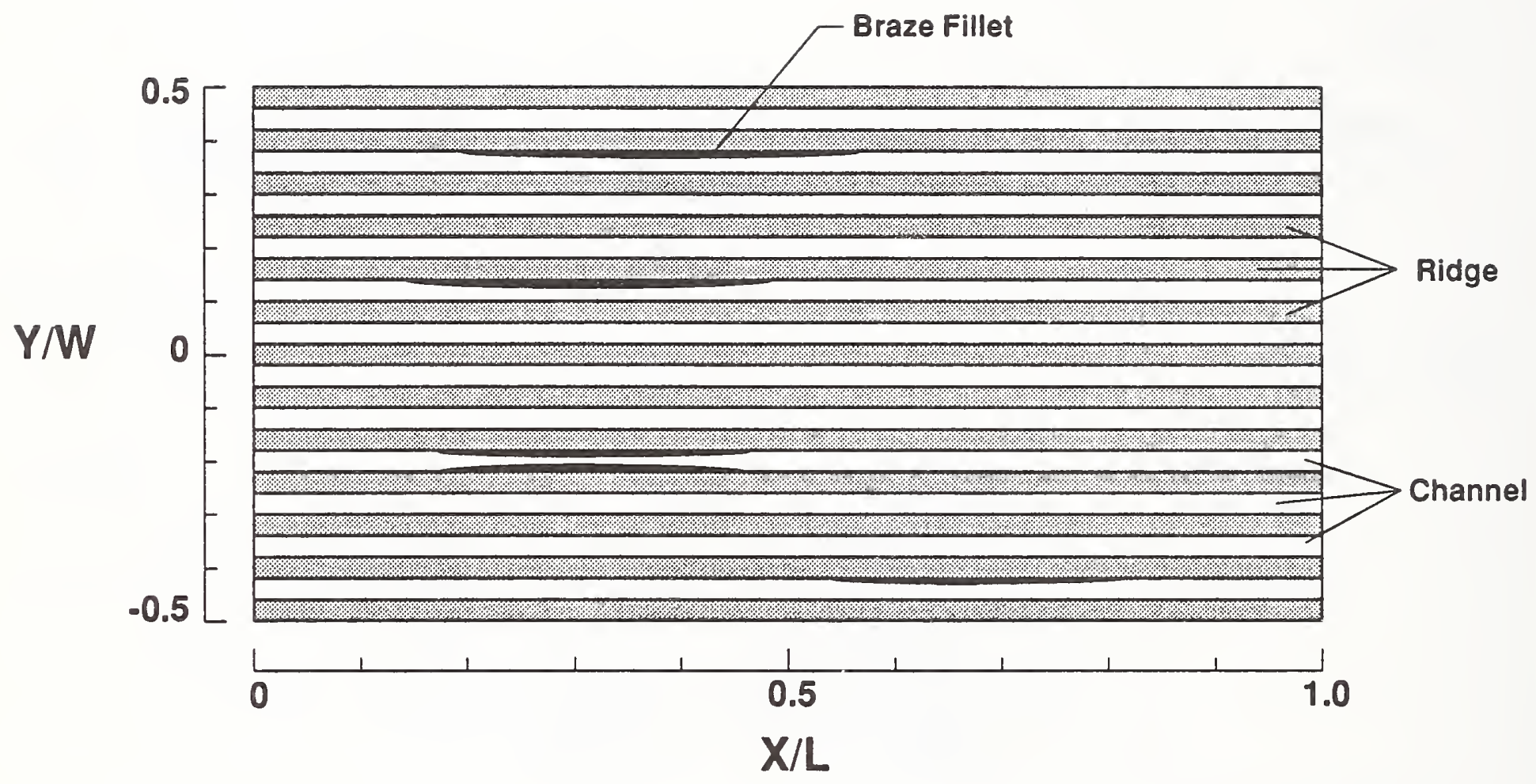

Figure 4. Top view of channel specimen showing locations of blockage in flow channels due to braze fillets. 


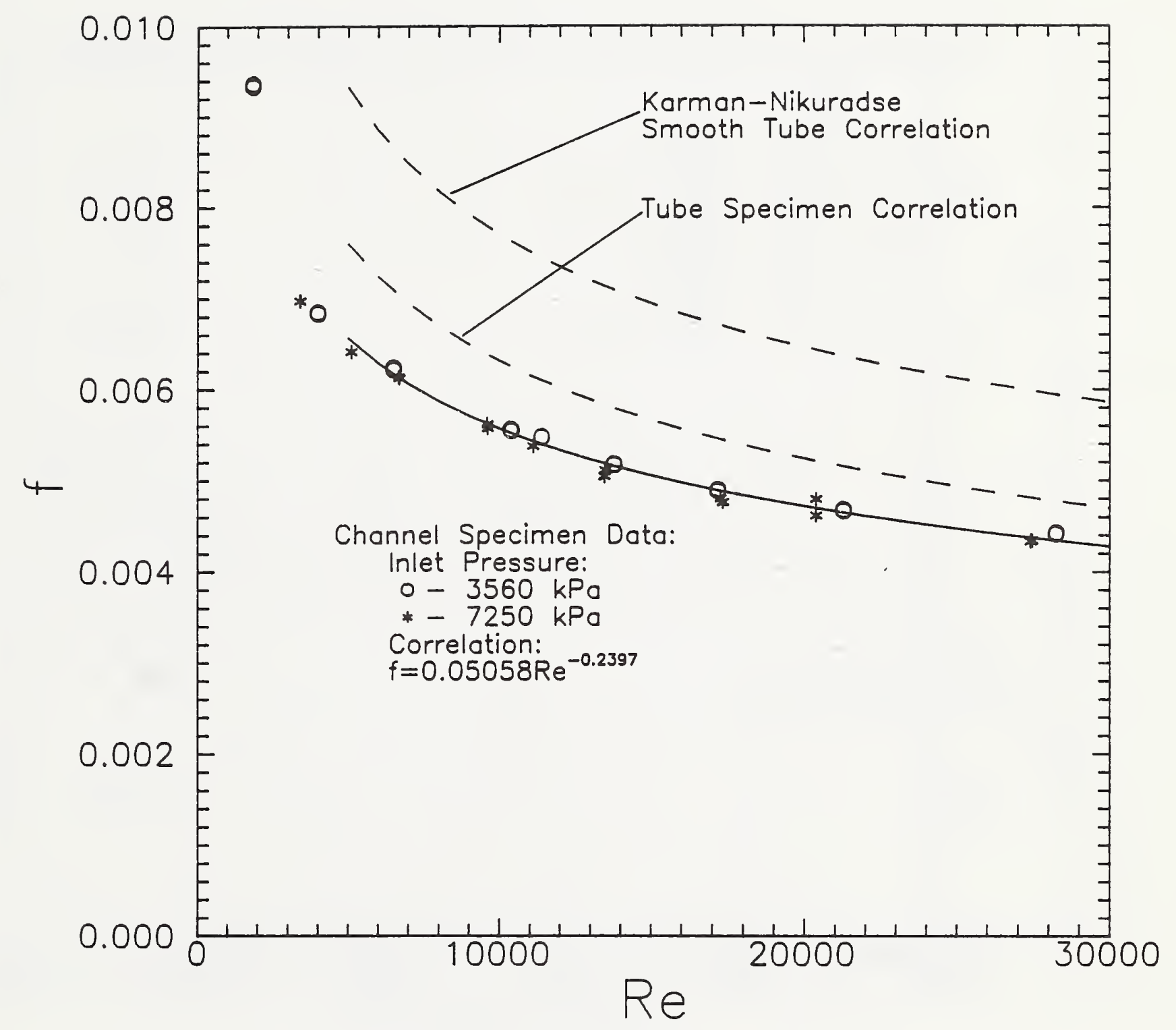

Figure 5. Friction factor ( $f$ ) as a function of Reynolds number (Re) for experiments 1 and 2 , no heating, compared to tube specimen correlation and smooth tube correlation. 


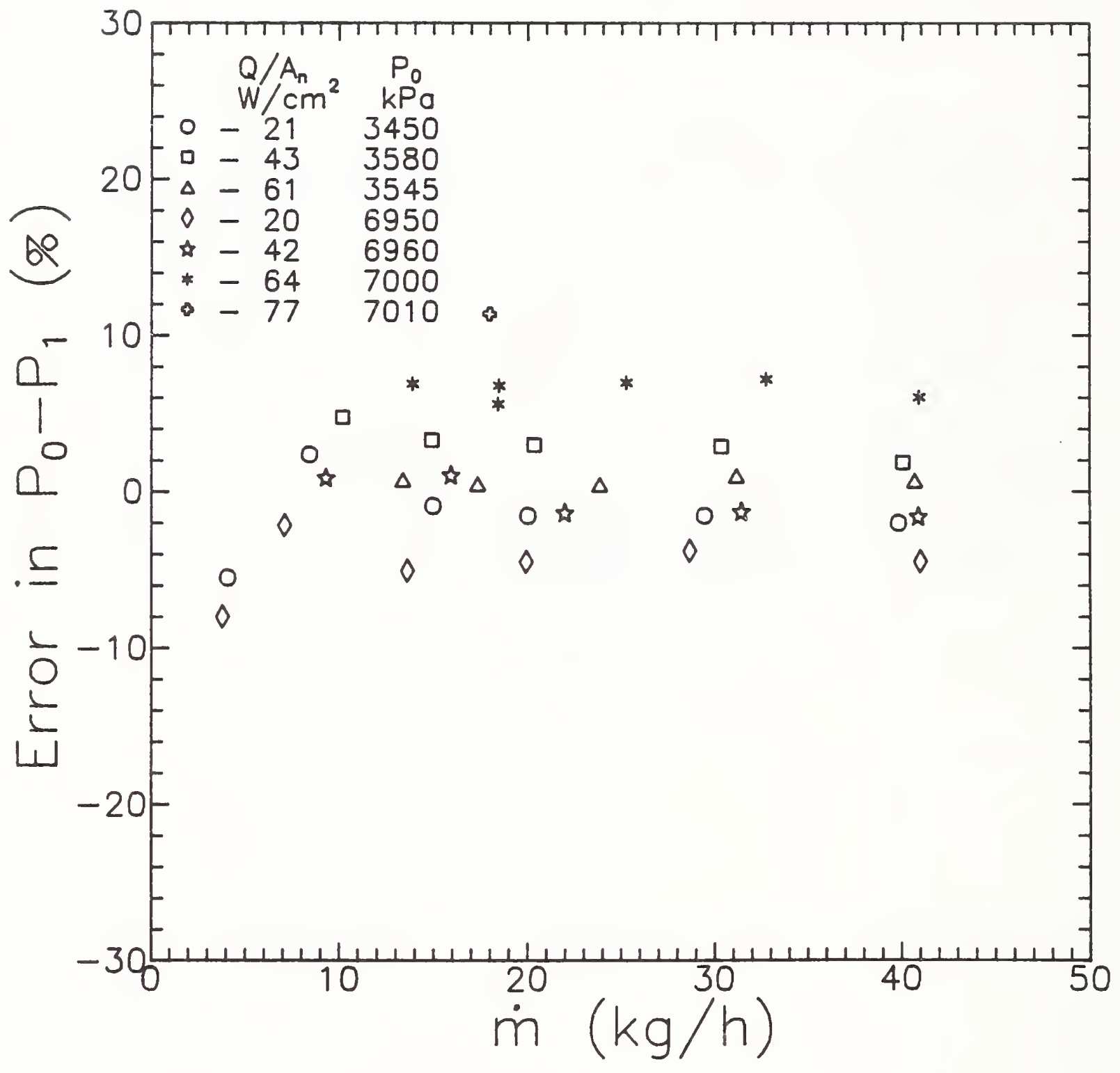

Figure 6. Percent difference between predicted and measured pressure drop $\left(P_{0}-P_{1}\right)$ as a function of helium flow rate $(\dot{m})$ for heat transfer experiments. 


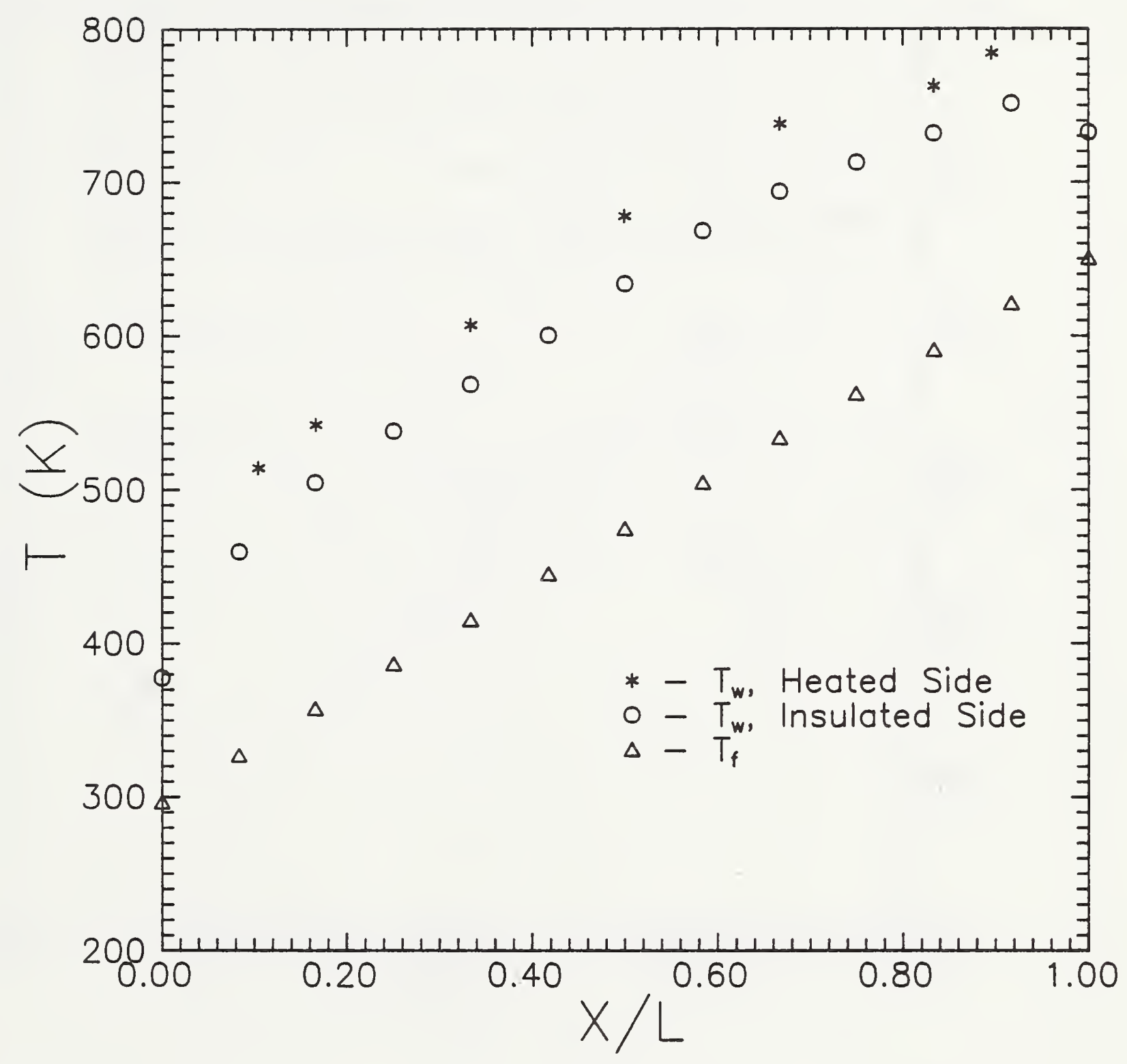

Figure 7. Wall $\left(T_{w}\right)$ and gas $\left(T_{f}\right)$ temperatures as a function of $x / L$; experiment $8,13.8 \mathrm{~kg} / \mathrm{h}$ helium flow, and $\mathrm{y} / \mathrm{W}=-0.04$. 


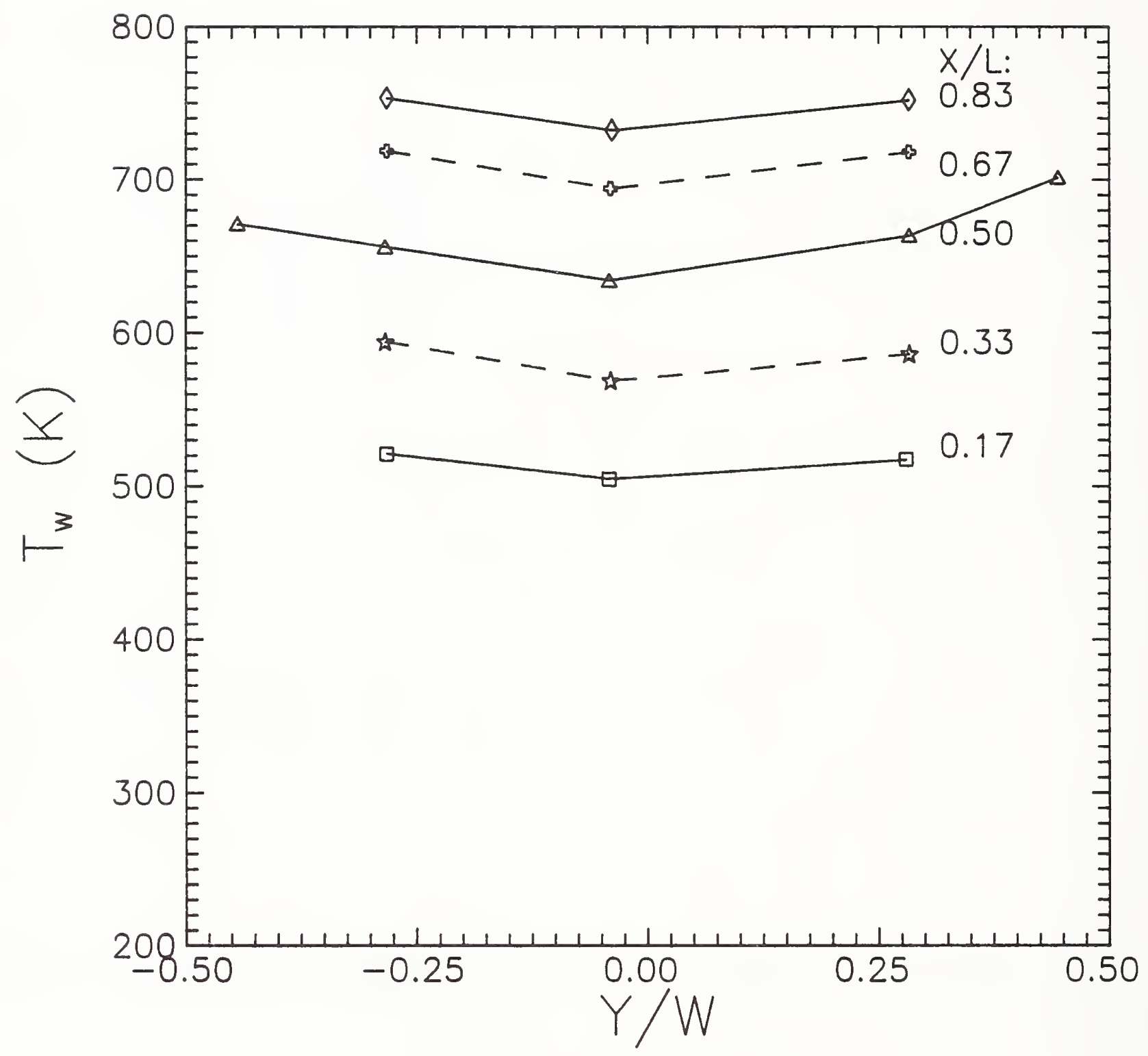

Figure 8. Wall temperature $\left(\mathrm{T}_{\mathrm{w}}\right)$ as a function of $\mathrm{y} / \mathrm{W}$ at several $\mathrm{x} / \mathrm{L}$ locations; experiment $8,13.8 \mathrm{~kg} / \mathrm{h}$ helium flow. 


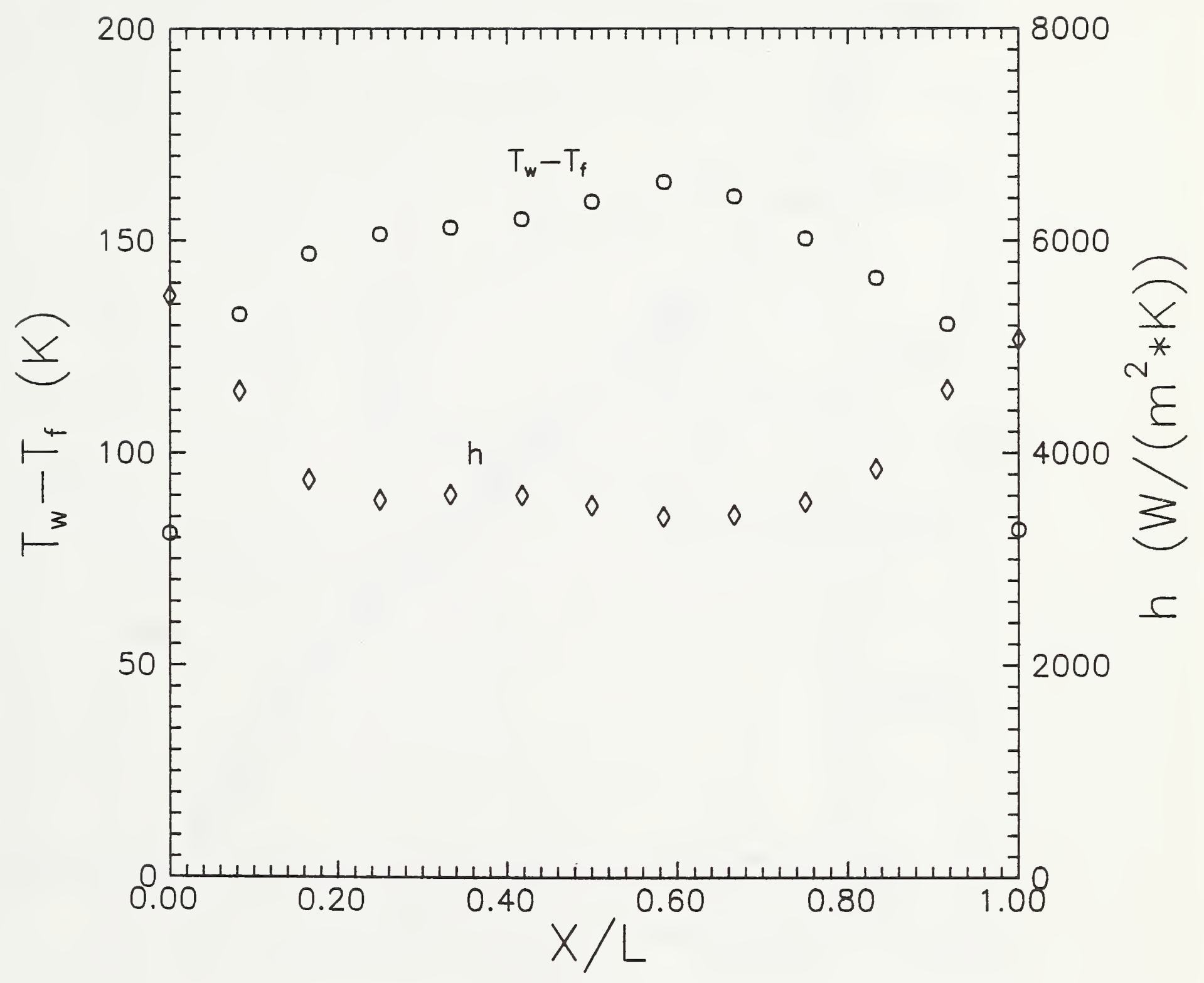

Figure 9. Wall-to-gas temperature difference $\left(T_{w}-T_{f}\right)$ and heat transfer coefficient $(\mathrm{h})$ as a function of $\mathrm{x} / \mathrm{L}$; experiment $8,13.8 \mathrm{~kg} / \mathrm{h}$ helium flow and $y / W=-0.04$. 


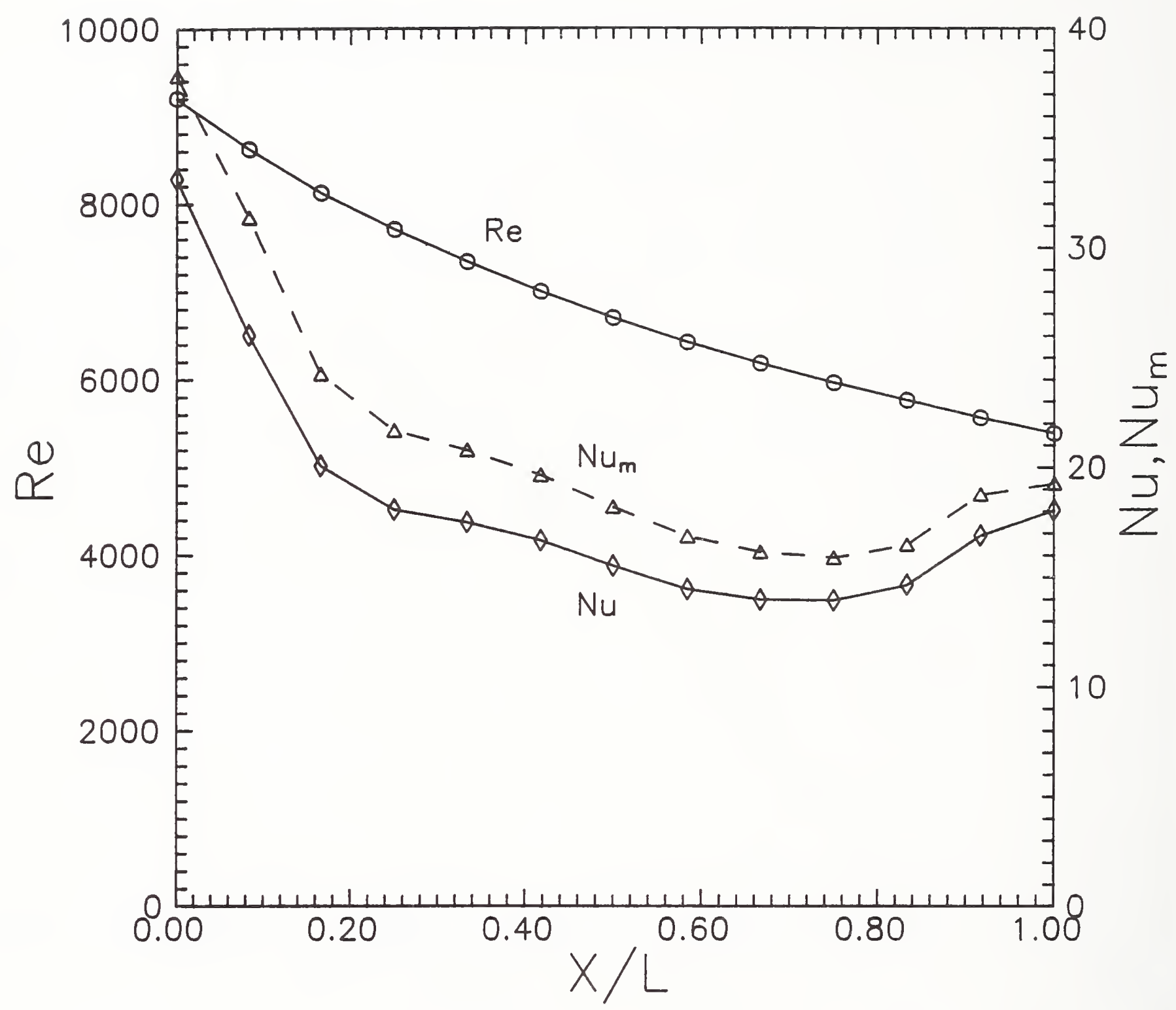

Figure 10. Reynolds number ( $R e$ ), Nusselt number ( $\mathrm{Nu}$ ), and modified Nusselt number $\left(\mathrm{Nu}_{\mathrm{m}}\right)$ as a function of $\mathrm{x} / \mathrm{L}$; experiment $8,13.8 \mathrm{~kg} / \mathrm{h}$ helium flow and $y / w=-0.04$. 


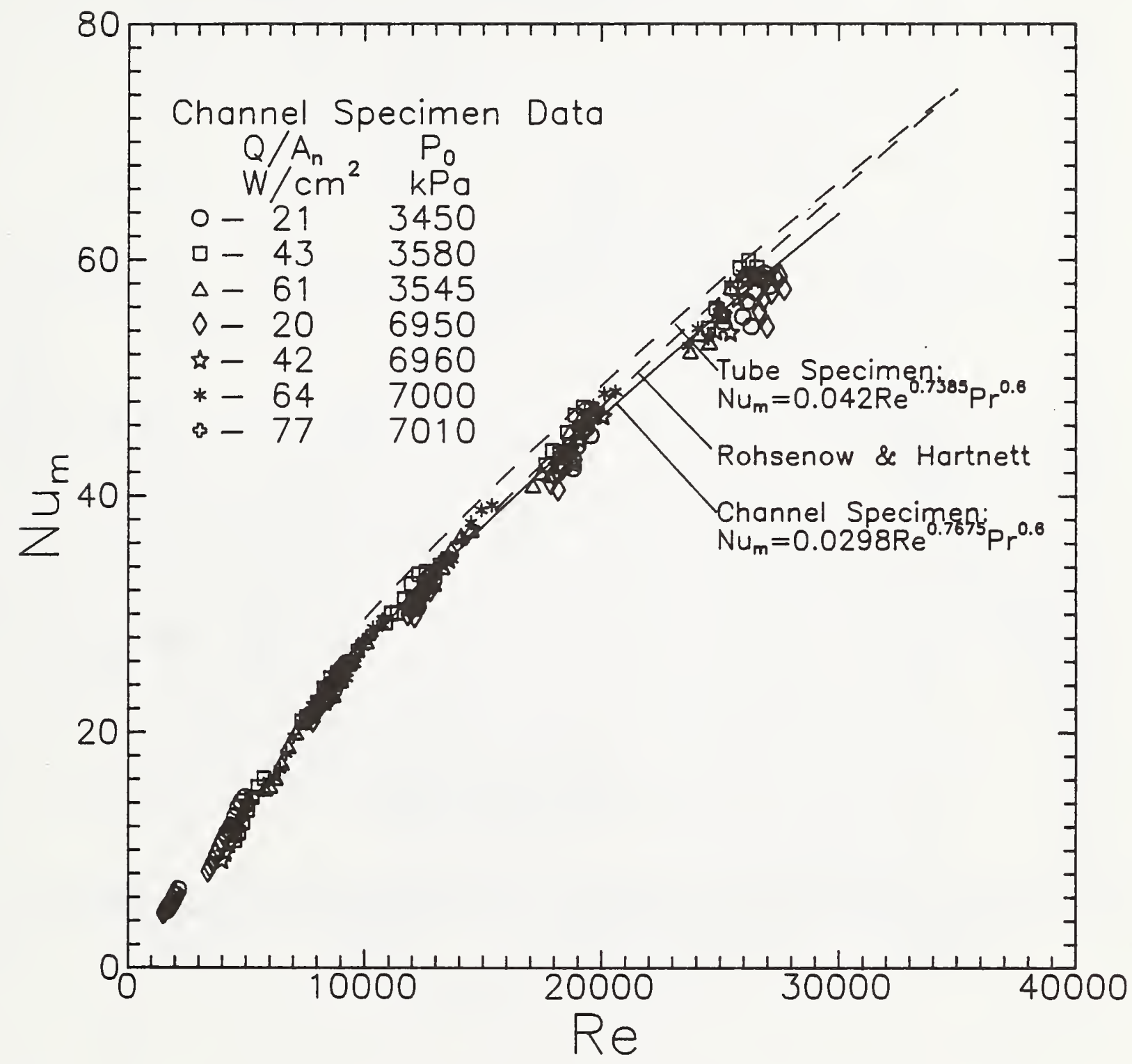

Figure 11. Modified Nusselt number $\left(\mathrm{Nu}_{\mathrm{m}}\right)$ as a function of Reynolds number $(\mathrm{Re}) ;$ all heated experiments with $0.2<\mathrm{x} / \mathrm{L}<0.8$ and $\mathrm{y} / \mathrm{W}=-0.04$. 


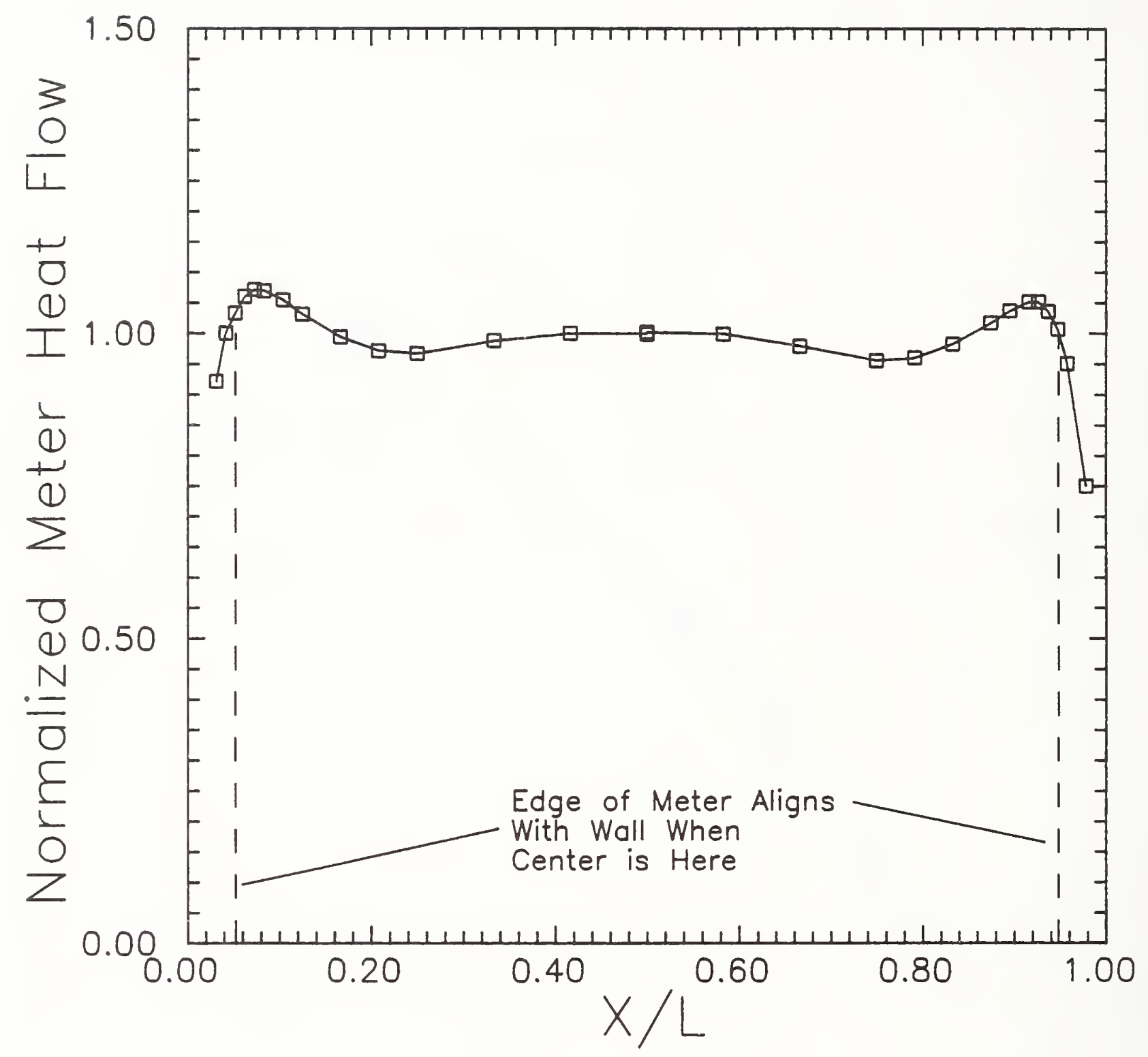

Figure A.1. Normalized meter heat flow as a function of $x / L$ for $y / W=0.12$, $36 \%$ voltage, reflective furnace calibration. 


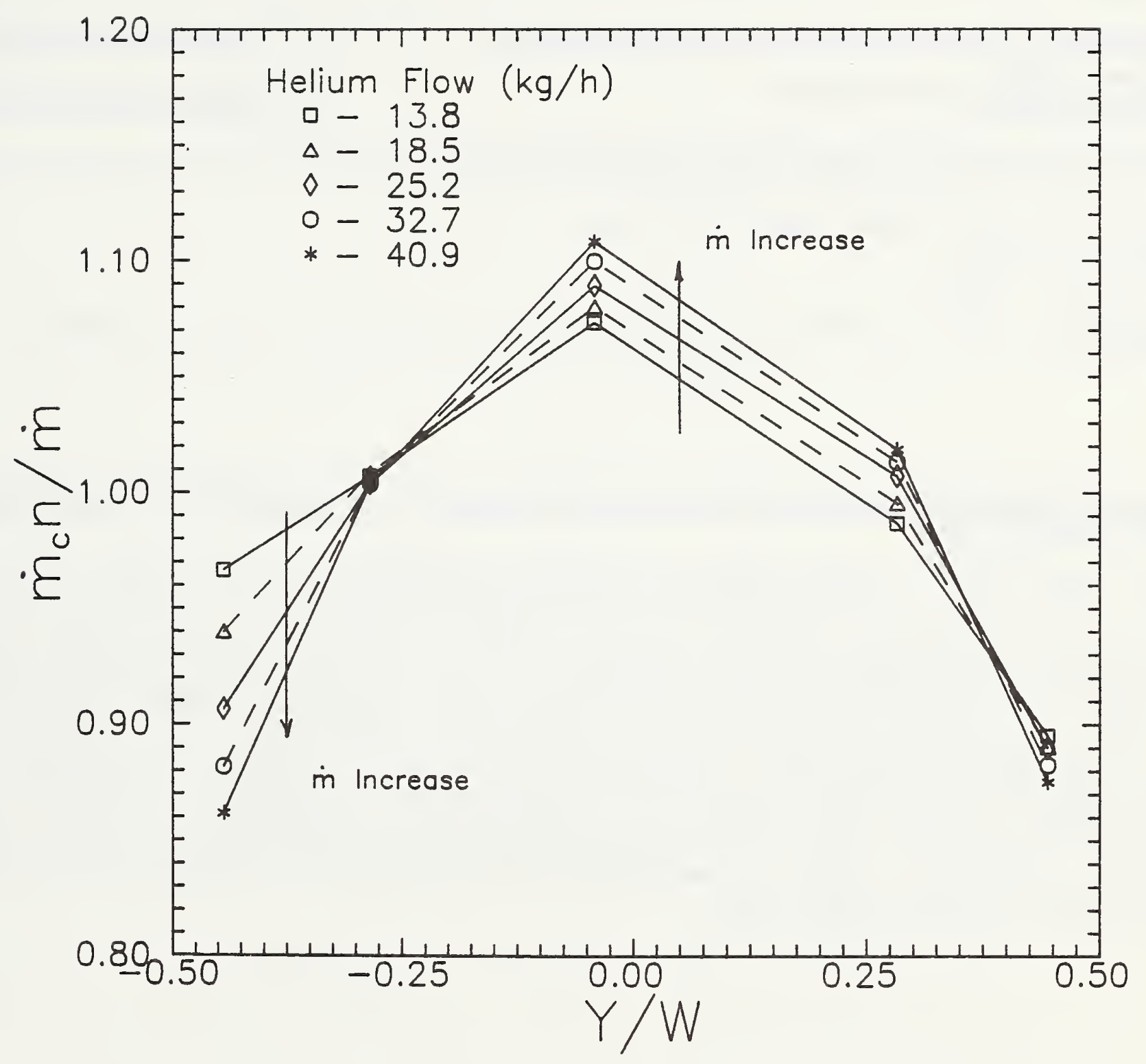

Figure B.1. Ratio of predicted channel flow to average channel flow, as a function of $\mathrm{y} / \mathrm{W}$ at several total flow rates for experiment 8 . 
4. TITLE AND SUBTITLE

HEAT TRANSFER IN A COMPACT HEAT EXCHANGER CONTAINING RECTANGULAR CHANNELS AND USING HELIUM GAS

5. AUTHOR(S)

Douglas A. Olson

6. PERFORMING ORGANIZATION (IF JOINT OR OTHER THAN NIST, SEE INSTRUCTIONS) U.S. DEPARTMENT OF COMMERCE MATIONAL INSTITUTE OF STANDARDS AND TECHNOLOQY BOULDER, COLORADO $80303-3328$

7. CONTRACT/GRANT NUMBER

8. TYPE OF REPORT AND PEAIOD COVERED

9. SPONSORING ORGANIZATION MAME AND COMPLETE ADDRESS (STREET, CITY, STATE, ZIP)

National Aeronautics and Space Administration

Langley Research Center

Hampton, Virginia 23665

10. SUPPLEMENTARY NOTES

11. ABSTRACT (A 200-WORD OR LESS FACTUAL SUMMARY OF MOST SIGNIFICANT INFORMATION. IF DOCUMENT INCLUDES A SIGNIFICANT BIBLOGRAPHY OR LTERATURE SURVEY, MENTION IT HERE.)

We have constructed a compact heat exchanger consisting of 12 parallel, rectangular channels in a flat piece of commercially pure nickel. ${ }_{2}$ This channel specimen was radiatively heated on the top side at heat fluxes of up to $77 \mathrm{~W} / \mathrm{cm}^{2}$ insulated on the back side, and cooled with helium gas flowing in the channels at 3.5 to $7.0 \mathrm{MPa}$ and Reynolds numbers of 1400 to 28 000. The measured friction factor was lower than that of the accepted correlation for fully developed turbulent flow, although our uncertainty was high due to uncertainty in the channel height and a high ratio of dynamic pressure to pressure drop. The measured Nusselt number, when modified to account for differences in fluid properties between the wall and the cooling fluid, agreed with past correlations for fully developed turbulent flow in channels. Flow nonuniformity from channel-to-channel was as high as $12 \%$ above and $19 \%$ below the mean flow.

12. KEY WORDS (6 TO 12 ENTRIES; ALPHABETICAL ORDER; CAPITALZE ONLY PROPER NAMES; AND SEPARATE KEY WORDS BY SEMICOLONS) apparatus; compact heat exchanger; convection heat transfer; friction factor; high temperature; National Aerospace Plane; radiative furnace; rectangular channel; turbulent flow; variable property effects.

\section{AVAILABILTY}

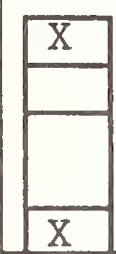

\section{UNUMITED}

FOR OFFICIAL DISTRIBUTION. DO NOT RELEASE TO NATIONAL TECHMICAL INFORMATION SERVICE (NTIS).

ORDER FROM SUPERINTENDENT OF DOCUNENTS, U.S. GOVERMMENT PRINTINO OFFICE, WASHINGTON, DC 20402.

ORDER FROM NATIONAL TECHNICAL INFORMATION SEAVICE (NTIS), SPRINGFIELD, VA 22161.
14. NUMBER OF PRINTED PAGES

$$
100
$$

15. PRICE 
